\title{
Wechselwirkungen von Gold und Versetzungen in Silizium
}

\author{
Dissertation \\ zur Erlangung des Doktorgrades \\ der Mathematisch-Naturwissenschaftlichen Fakultäten \\ der Georg-August-Universität zu Göttingen
}

vorgelegt von

Oliver Voß

aus

Oldenburg

Göttingen 2009 
D 7

Referent: Herr Prof. Dr. M. Seibt

Korreferent: Herr Prof. Dr. C. Jooß

Tag der mündlichen Prüfung: 28. Mai 2009 


\section{Inhaltsverzeichnis}

1 Einleitung 1

2 Einführung 5

2.1 Experimentelle Methoden . . . . . . . . . . . . . . . 5

2.1.1 Kapazitätstransientenspektroskopie tiefer Zustände . . . . . . 5

2.1.2 DLTS an Vielelektronendefekten . . . . . . . . . . . . . . 9

2.1.2.1 Modell zur Beschreibung von DLTS-Spektren ausgedehn-

ter Vielelektronendefekte . . . . . . . . . . . . . . 11

2.2 Gold in Silizium . . . . . . . . . . . . . . . . . . . . . . 12

2.2.1 Diffusion und Löslichkeit . . . . . . . . . . . . . . . . . . . 12

2.2.2 Ausscheidungen . . . . . . . . . . . . . . . . . . . . . . . . . . . . . . . .

2.2 .3 Tiefe Zustände . . . . . . . . . . . . . . . . . . . . . . . 16

2.3 Versetzungen in Silizium . . . . . . . . . . . . . . 17

2.3.1 Strukturelle Eigenschaften . . . . . . . . . . . . . 17

2.3.1.1 Das Gleitsystem im Siliziumkristall . . . . . . . . . . . 17

2.3.1.2 Das elastische Verzerrungsfeld . . . . . . . . . . . . . . . 18

2.3.2 Elektrische Eigenschaften . . . . . . . . . . . . . . . 18

2.3.2.1 Effekt der Linienladung . . . . . . . . . . . . . . . 19

2.3.2.2 Das elastische Verzerrungsfeld . . . . . . . . . . . . . 20

2.4 Wechselwirkungen metallischer Verunreinigungen mit Versetzungen . . . 22

2.4.1 Chemische und strukturelle Wechselwirkungen . . . . . . . . . . 22

2.4.2 Elektrische Wechselwirkungen . . . . . . . . . . . . . 23

3 Probenpräparation 27

3.1 Allgemeines . . . . . . . . . . . . . . . . . . 27

3.2 Erzeugung von Versetzungen . . . . . . . . . . . . . . . . . 28

3.3 Probenentnahme aus den deformierten Stäbchen . . . . . . . . . . . 28

3.4 Goldeindiffusion . . . . . . . . . . . . . . . . . . . . . . 29

3.5 Schrägschliff . . . . . . . . . . . . . . . . . . . 30

3.6 Kontaktpräparation . . . . . . . . . . . . . . . . . . 30

3.7 Defektätzen an verformten Proben . . . . . . . . . . . . . 31

3.8 Präparation der TEM-Probe . . . . . . . . . . . . . . . . . 32 
4 Ergebnisse $\quad 35$

4.1 Versetzungsdichten . . . . . . . . . . . . . . . 35

$4.1 .1 \quad$ DLTS-Proben . . . . . . . . . . . . . . . . . . . 36

4.1.2 Die unterschiedlich ausgelagerten Proben . . . . . . . . . . . . . 36

4.1.3 Versetzungsdichte der Probe einer anderen Veröffentlichung . . . . 37

4.2 Ergebnisse der DLTS-Messungen . . . . . . . . . . . . . . . . 39

4.2.1 Gold in versetzungsfreiem Silizium . . . . . . . . . . . . . . 39

4.2.1.1 n-dotiertes Silizium . . . . . . . . . . . . . . . 39

4.2.1.2 p-dotiertes Silizium . . . . . . . . . . . . . 43

4.2.2 Gold in versetzungshaltigem Silizium . . . . . . . . . . . . 48

4.2.2.1 n-dotiertes Silizium . . . . . . . . . . . . . . 49

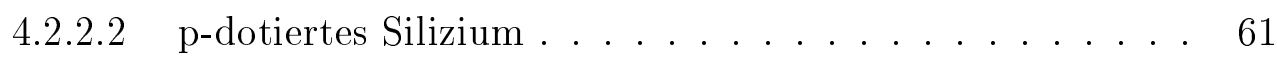

4.3 Goldausscheidungen . . . . . . . . . . . . . . . . . 72

5 Diskussion $\quad 75$

5.1 Elektrische Eigenschaften golddekorierter Versetzungskerne . . . . . . . 76

5.1.1 Identifikation der goldinduzierten DLTS-Linien . . . . . . . . . . . 76

5.1.2 Einfluss der Besetzung tiefer Zustände auf die Dichte freier Ladungsträger . . . . . . . . . . . . . 78

5.1.3 Identifikation, Besetzung und Linienladung der Versetzungszustände 81

5.1.4 Das Versetzungspotential als Einfangbarriere . . . . . . . . . . . . 83

5.2 Diskussion anderer Modelle zur Beschreibung der modifizierten Einfang-

kinetik in versetzungshaltigem Silizium . . . . . . . . . . . . 90

5.3 Konzentrationen von Gold in versetzungshaltigem Silizium . . . . . . . . 91

5.3.1 Ergebnisse einer anderen Veröffentlichung und deren Interpretation 91

5.3.2 Alternative Interpretation der Ergebnisse einer anderen Veröffentlichung . . . . . . . . . . . . . . . . . 94

5.4 Einfluss der Versetzungen auf die Golddiffusion . . . . . . . . . . . . . 99

5.5 Auffälligkeit in den DLTS-Spektren der versetzungshaltigen p-dotierten

Probe ........................ . . 100

6 Zusammenfassung und Ausblick

Anhänge

A Modell zur Berechnung von Punktdefektkonzentrationen 107

B Gold im Verzerrungsfeld von Versetzungen

C Das Phasendiagramm des Systems Gold/Silizium 113

\begin{tabular}{ll} 
Literaturverzeichnis & 115 \\
\hline
\end{tabular} 


\section{Einleitung}

Der Verbrauch fossiler Energieträger erfordert in Zukunft immer kostenintensivere Maßnahmen für deren Erschließung. Gleichzeitig werden die Beeinträchtigungen der Umwelt aufgrund der Nutzung fossiler Energieträger offensichtlich. Somit sollte ein großes Interesse an der Nutzung nachhaltiger Energiequellen bestehen. Die Akzeptanz für eine nachhaltige Entwicklung auf dem Energiesektor hängt dennoch von den Kosten ab, die für Energie aus nachhaltigen Quellen, wie dem Sonnenlicht oder dem Wind, aufgebracht werden müssen.

Unterstützt durch staatliche Subventionsprogramme konnten die Kosten für die Gewinnung elektrischer Energie aus dem Sonnenlicht, der Photovoltaik, deutlich reduziert werden. Durch den seit einigen Jahren einsetzenden Übergang zur Massenproduktion von Solarzellen und Photovoltaikmodulen besteht die Möglichkeit, die Kosten weiter zu senken.

Da sich die Rentabilität des Produktes Solarzelle in den Kosten für die Herstellung einer Solarzelle mit einer bestimmten Leistung bemisst $\left(\$ / \mathrm{W}_{\mathrm{p}}, \mathrm{W}_{\mathrm{p}}\right.$ : „Watt-Peak“, unter Standardbedingungen erzielte Leistung einer Solarzelle $\left.{ }^{[1]}\right)$, besteht ein Kostenoptimierungspotential auch in der Steigerung der Leistung einer Solarzelle. Hier kann die Forschung an Universitäten und anderen Forschungsinstituten sowie die industrielle Forschung einen wichtigen Beitrag leisten.

Im Jahr 2006 machte die siliziumbasierte Photovoltaiktechnologie über 90\% des Gesamtmarktes aus ${ }^{[2]}$. Mehr als $50 \%$ davon fallen auf auf das kostengünstigere multikristalline Silizium ${ }^{[3]}$. Neben kristallographischen Defekten, wie den Korngrenzen und Versetzungen, enthält dieses Material nichtmetallische und metallische Vereunreinigungen. $\mathrm{Zu}$ den häufigsten metallischen Verunreinigungen zählen Eisen, Kupfer und Nickel ${ }^{[\underline{3}-\underline{5}}$.

Metallische Verunreinigungen in Silizium sind mit Energiezuständen verbunden, die tief in der Bandlücke liegen ${ }^{[6]}$. Diese tiefen Zustände in der Bandlücke wirken als effektive Rekombinationszentren für Elektronen und Löcher ${ }^{[7.8]}$ und reduzieren auf diese Weise die Lebensdauer von Minoritätsladungsträgern, die in Solarzellen als Überschussladungsträger durch Lichtabsorbtion erzeugt werden. Da diese Minoritätsladungsträgerlebensdauer einen maßgeblichen Faktor für den Wirkungsgrad einer Solarzelle darstellt, wirkt sich eine hohe Dichte gelöster metallischer Verunreinigungen schädlich auf die Leistung einer Solarzelle aus ${ }^{[9]}$. Daher wird im Rahmen notwendiger Prozessschritte während der Herstellung versucht, die Dichte dieser schädlichen Verunreinigungen durch Umverteilung zu reduzieren. Zwei Möglichkeiten stehen für diese sogenannte Defektmanipulation prinzipiell zur Verfügung: Zum einen können die Fremdatome in elektrisch inaktive Bereiche 
gebracht werden ${ }^{[10]}$, das sind bei der Solarzelle der Emitter und der Rückseitenkontakt. Eine andere Möglichkeit besteht darin, möglichst viele Fremdatome in möglichst wenigen Ausscheidungen zu sammeln, um den Abstand rekombinationsaktiver Defekte zu reduzieren und damit die Diffusionslänge der Minoritätsladungsträger zu erhöhen $\underline{\text { [11] }}$.

Korngrenzen und Versetzungen besitzen ebenfalls eine für Solarzellen schädliche Wirkung. So konnte zwar gezeigt werden, dass Versetzungen, die nur schwach mit metallischen Verunreinigungen dekoriert waren, kaum rekombinationsaktiv sind. Die Rekombinationsaktivität wuchs jedoch deutlich mit dem Grad der Kontamination mit metallischen Fremdatomen ${ }^{[12]}$. Die Annahme, dass derartige Kontaminationen aber auch intrinsische Defekte, die mit Versetzungen in Verbindung stehen, die Rekombinationsaktivität der Versetzungen steigern, konnte durch zahlreiche Experimente untermauert werden (Zusammenfassung bei SCHRÖTER UND CERvA ${ }^{[13]}$ ). An dieser Stelle zeigt sich, dass grundlegende Kenntnisse über die Wechselwirkungen von kristallographischen Defekten und metallischen Verunreinigungen wichtig für das Verständnis schädlicher Einflüsse auf den Wirkungsgrad von Solarzellen sind.

Wechselwirkungen dieser Art können in unterschiedlicher Weise und in unterschiedlichen Bereichen in der Umgebung von Versetzungen stattfinden: Im Versetzungskern, der einen radialen Bereich von ca. einer Gitterkonstanten (in Silizium ca. 5.4A) umfasst, ist die Symmetrie des Kristallgitters gestört. Daher unterscheiden sich die chemischen Bindungen und die elektrischen Eigenschaften metallischer Fremdatome hier deutlich von denen in Bereichen zwischen den Versetzungen. Theoretische Berechnungen von Bindungsenergien einzelner Kupfer- oder Goldatome im Versetzungskern lassen hier hohe Bindungsenergien (1-2eV) erwarten $\left.{ }^{[14}, 15\right]$. Derartige Bindungsenergien können zu beträchtlichen Akkumulationen von Fremdatomen im Versetzungskern führen ${ }^{[16]}$. Ob und in welcher Weise Fremdatome mit großen Liniendichten im Versetzungskern miteinander wechselwirken, ist bisher ebenso wenig untersucht, wie deren Einfluss auf die Umgebung der Versetzungen.

Einen etwas weiteren Bereich möglicher Wechselwirkungen umfasst das Verzerrungsfeld $\left(1-2 n m^{[13]}\right)$ einer Versetzung. Hier können Fremdatome vorwiegend aufgrund des Größenunterschiedes ihrer kovalenten Radien zu denen der Siliziumatome gebunden wer-

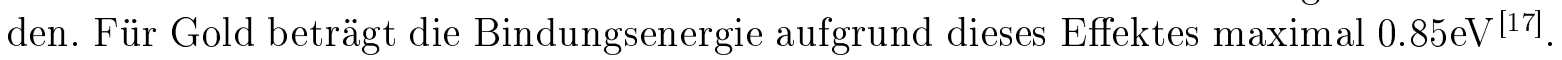
Da sich die Energieniveaus der Bandkanten und die mit den Fremdatomen assoziierten tiefen Zustände im Verzerrungsfeld aufgrund unterschiedlicher Deformationspotentiale unterschiedlich gegeneinander verschieben können ${ }^{[13]}$, kann dies Auswirkungen auf die elektrischen Eigenschaften der hier befindlichen tiefen Zustände haben ${ }^{[18]}$.

Schließlich ist ein noch weiträumigeres Gebiet möglicher Wechselwirkungen zwischen Versetzungen und metallischen Verunreinigungen zu betrachten: Geladene Versetzungszustände können mit einem elektrostatischem Potential, das einen zylindersymmetrischen Bereich mit einem Radius von einigen 100nm umfassen kann, die Dichte freier Ladungsträger abstandsabhängig erhöhen oder reduzieren [19]. Dies kann ebenfalls zu veränderten elektrischen Eigenschaften aufgrund modifizierter Einfangkinetiken der tiefen 
Zustände führen. Allerdings gibt es auch zu diesem Effekt bisher keine Untersuchungen.

Diesen Fragen widmet sich die vorliegende Arbeit. Dazu wurden insbesondere elektrische Eigenschaften untersucht, die Goldatome im versetzungshaltigen Silizium in unterschiedlichen Bindungszuständen besitzen. Es stellte sich heraus, dass im Versetzungskern gebundene Goldatome Zustände in die Bandlücke des Siliziums einführen, deren Besetzung mit Elektronen ein elektrostatisches Potential mit Wirkung auf umliegende, im Gitter zwischen den Versetzungen gelöste Goldatome bewirkt.

Gold eignet sich aus verschiedenen Gründen zur Untersuchung derartiger Wechselwirkungen metallischer Fremdatome mit Versetzungen: Zum einen sind die Eigenschaften von Gold in versetzungsfreiem Silizium hinlänglich bekannt. Auf diese wird im Kap. 2.2 ausführlich eingegangen. Zum anderen lassen sich wegen der Diffusions- und Löslichkeitseigenschaften Konzentrationsprofile einstellen, die die Untersuchung der Konzentrationsabhängigkeit der beobachteten Effekte erleichtert. Dies wurde insbesondere auch für versetzungshaltiges Silizium gezeigt, wo eine gegenüber versetzungsfreiem Silizium deutlich erhöhte Gesamtgoldkonzentration beobachtet werden konnte ${ }^{[20]}$.

Darüber hinaus besitzt Gold in Silizium eine tiefe Störstelle sowohl in der oberen als auch in der unteren Hälfte der Energiebandlücke, so dass dieses System für die gewählte Messmethode der Kapazitätstransientenspektroskopie (DLTS, „Deep Level Transient Spectroscopy“, Kap. 2.1) zumindest in versetzungsfreiem Silizium beider Dotierungsarten (p- und n-Dotierung) Ergebnisse verspricht, die mit denen von versetzungshaltigem verglichen werden können. Versetzungen können, wie oben erwähnt, sowohl aufgrund ihrer strukturellen als auch wegen ihrer elektrischen Eigenschaften das Verhalten metallischer Verunreinigungen beeinflussen. Diese Eigenschaften werden im Kap. 2.3 zusammengefasst.

Für das Verständnis der Ergebnisse in Kap. 4 und deren Diskussion im Kap. 5 sind gewisse Grundlagen über die Wechselwirkungen metallischer Fremdatome mit Versetzungen erforderlich. Diese werden im letzten Abschnitt des Einführungskapitels (Kap. 2.4) beschrieben.

Da der Einfluss der Goldkonzentration auf die Messergebnisse untersuchen werden sollte, waren spezielle Präparationsschritte notwendig. Außerdem war für die Interpretation der Ergebnisse die Höhe der Versetzungsdichte von Bedeutung. Daher wird im Kap. 3 genau auf jeden Präparationsschritt eingegangen.

Im Kap. 6] werden die wichtigsten Erkenntnisse zusammengefasst und offene Fragen in Zusammenhang mit weiteren möglichen Experimenten formuliert.

Die vorliegende Arbeit entstand im Rahmen des vom BMU geförderten Verbundprojektes ,Solarfocus“. Ziel dieses Forschungsprojektes ist es, ein umfassendes Verständnis der Eigenschaften verschiedener Defektarten in kristallinem Silizium mit Relevanz für die Photovoltaik zu erhalten 


\section{Einführung}

$\mathrm{Zu}$ Beginn werden die in dieser Arbeit verwendeten experimentellen Methoden beschrieben. Dabei handelt es sich zunächst um die Kapazitätstransientenspektroskopie (DLTS, „Deep Level Transient Spectroscopy“). Da diese in der Literatur hinreichend beschrieben ist, werden im Abschnitt 2.1.1 nur die Grundlagen dieses Verfahrens sowie die Besonderheiten bei der Beschreibung von DLTS an Vielelektronensystemen erläutert. Im darauf folgenden Abschnitt 2.1.2.1 wird das Modell erklärt, auf dessen Grundlage die Eigenschaften der tiefen Zustände durch numerische Anpassung bestimmt wurden. Anschließend folgt die Darstellung der für diese Arbeit relevanten Grundlagen über Gold und Versetzungen sowie deren Wechselwirkung mit metallischen Verunreinigungen in Silizium (Abschnitte 2.2, 2.3, 2.4).

\subsection{Experimentelle Methoden}

\subsubsection{Kapazitätstransientenspektroskopie tiefer Zustände}

Die DLTS ist eine von LANG ${ }^{[21]}$ entwickelte Methode, mit dem Eigenschaften der von Gitterfehlern generierten tiefen Zustände untersucht werden können. Dabei wird die Kapazitätsänderung der Raumladungsschichten von Halbleiterdioden - in dieser Arbeit handelt es sich um Schottky-Kontakte - gemessen, die durch Umladen der tiefen Zustände hervorgerufen wird. Das Umladen erfolgt durch Überlagern einer am Schottky-Kontakt angelegten Sperrspannung $U_{\mathrm{b}}$ mit einem periodischen Spannungspuls $U_{\mathrm{p}}$ der Dauer $t_{\mathrm{p}}$ mit der Pulswiederholfrequenz $f_{\mathrm{W}}$. In Abb. 2.1 bezeichnen $U_{1}=U_{\mathrm{D}}+U_{\mathrm{b}}-U_{\mathrm{p}}$ das Potential am Rand der Raumladungszone während des Ladungspulses und $U_{0}=U_{\mathrm{D}}+U_{\mathrm{b}}$ das Potential zwischen den Ladungspulsen.

Während des Ladungspulses, der die Raumladungszone $W_{0}$ auf eine durch $U_{\mathrm{b}}-U_{\mathrm{p}}$ festgelegte Breite $W_{1}$ reduziert, wird eine neue Besetzung der tiefen Zustände durch den Einfang freier Ladungsträger eingestellt. Nach dem Ende dieser Einfangphase erfolgt die Relaxation durch thermische Emission der zuvor eingefangenen Ladungsträger. In der folgenden Beschreibung werden die wichtigsten theoretischen Grundlagen der DLTS-Messmethode betrachtet. Für detailliertere Betrachtungen sei auf die Literatur verwiesen $\stackrel{[21-23]}{2}$.

Unter der Annahme, dass der Besetzungsgrad innerhalb der Raumladungszone nicht vom Ort abhängt, ist der zeitabhängige Besetzungsgrad eines tiefen Zustands gegeben 

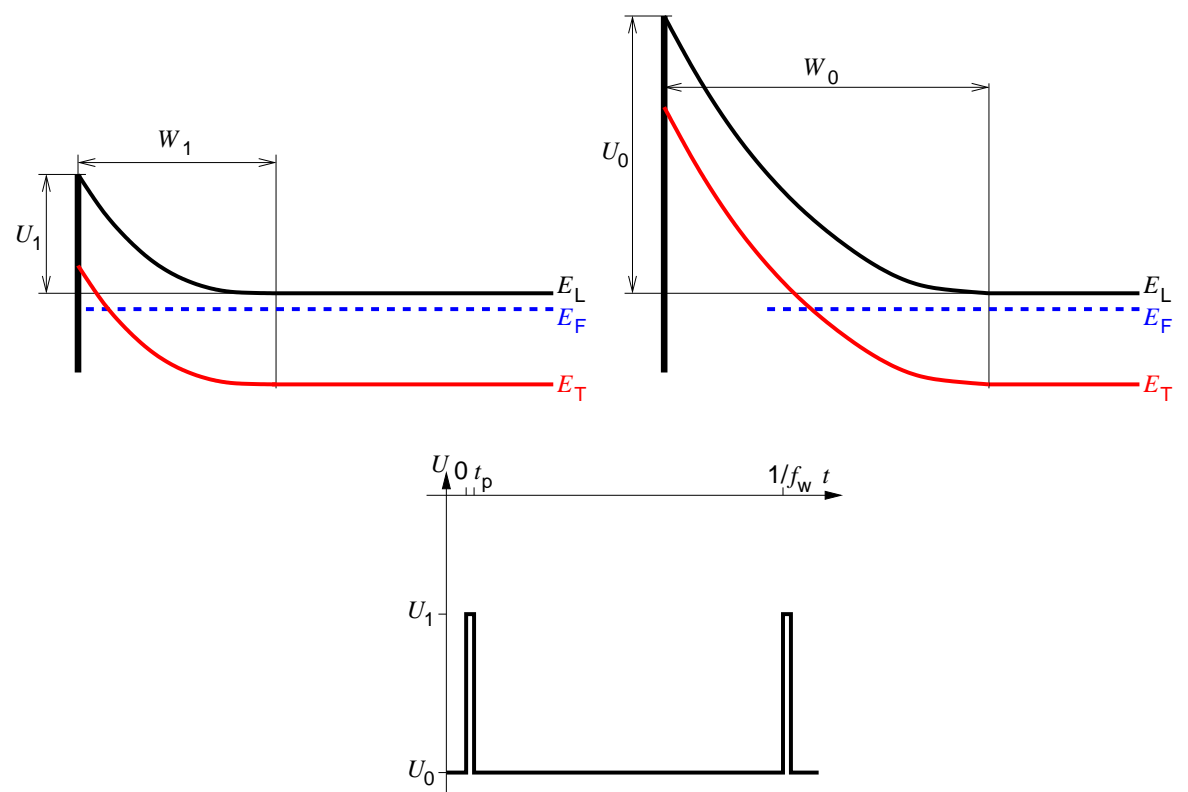

Abbildung 2.1 - Die Raumladungszone eines n-dotierten Halbleiters mit dem Potential $U_{1}$ am Rand der Raumladungszone während des Ladungspulses (links) und mit dem Potential $U_{0}$ zwischen den Ladungspulsen (rechts). $W_{1}$ und $W_{0}$ bezeichnen die entsprechenden Ausdehnungen der Raumladungszone und $E_{\mathrm{L}}, E_{\mathrm{F}}$ und $E_{\mathrm{T}}$ die Leitungsbandkante, das Fermi-Niveau und die Energie des tiefen Zustands. Unten ist der Verlauf des am Rand der Raumladungszone anliegenden Potentials über der Zeit während einer Messperiode dargestellt.

durch

$$
f(t)=\frac{N_{\mathrm{T}}^{+/-}(t)}{N_{\mathrm{T}}}
$$

wobei $N_{T}^{+/-}$und $N_{T}$ die Dichte der geladenen Zustände bzw. deren Gesamtdichte darstellen. Die zeitliche Änderung der Besetzung wird durch die Ratengleichung

$$
\frac{\mathrm{d} f(t)}{\mathrm{d} t}=\left(c_{n} n+e_{p}\right)(1-f(t))-\left(e_{n}+c_{p} p\right) f(t)
$$

beschrieben. Dabei sind

$$
c_{n} n=\sigma_{n}\left\langle v_{\text {th }}\right\rangle n
$$


die Einfangrate der Elektronen mit der Dichte freier Ladungsträger $n$ und

$$
\begin{aligned}
e_{n}(T) & =\sigma_{n} \gamma_{n} T^{2} \chi_{n} \exp \left(-\frac{\Delta H_{n}}{k_{\mathrm{B}} T}\right) \text { mit } \\
\gamma_{n} T^{2} & =\left\langle v_{\mathrm{th}, n}\right\rangle N_{\mathrm{L}} \text { und } \\
\chi_{n} & =\exp \left(\frac{\Delta S_{n}}{k_{\mathrm{B}}}\right)
\end{aligned}
$$

deren thermische Emissionsrate. $\sigma_{n}$ stellt den Einfangquerschnitt und $\left\langle v_{\text {th }}\right\rangle$ die mittlere thermische Geschwindigkeit der Ladungsträger dar. $N_{\mathrm{L}}$ ist die effektive Zustandsdichte im Leitungsband, $\Delta S$ die Entropieänderung und $\Delta H_{n}$ die Aktivierungsenthalpie des Defektes. Entsprechendes gilt für den Einfang von Löchern $p$ aus und deren Emission in das Valenzband mit der effektive Zustandsdichte $N_{V}$.

Die allgemeine Lösung der Ratengleichung (2.2) lautet:

$$
f(t)=\frac{c_{n} n+e_{p}}{c_{n} n+e_{p}+e_{n}+c_{p} p}+\left(f(0)-\frac{c_{n} n+e_{p}}{c_{n} n+e_{p}+e_{n}+c_{p} p}\right) \mathrm{e}^{-\left(c_{n} n+e_{p}+e_{n}+c_{p} p\right) t}
$$

Während der Emissionsphase vereinfacht sich Gl. (2.2) aufgrund des zu vernachlässigenden Einfangs von Ladungsträgern zu

$$
f(t)=\frac{e_{p}}{e_{p}+e_{n}}+\left(f(0)-\frac{e_{p}}{e_{p}+e_{n}}\right) \mathrm{e}^{-\left(e_{p}+e_{n}\right) t} .
$$

Somit entspricht $f_{\infty}=\frac{e_{p}}{e_{p}+e_{n}}$ der Besetzung des stationären Zustandes. Die Emission und der instantan erfolgenden Abtransport der Ladungsträger aus der Raumladungszone führt zu einer Kapazitätsänderung, die für kleine Dichten tiefer Zustände $\left(N_{\mathrm{T}} \ll N_{\mathrm{D} / \mathrm{A}}\right.$, $N_{\mathrm{D} / \mathrm{A}}$ : Dichte der flachen Donatoren bzw. Akzeptoren) in erster Näherung mit

$$
\begin{aligned}
\frac{2 \Delta C(t)}{C_{0}} \cdot N_{\mathrm{D} / \mathrm{A}} & =-N_{\mathrm{T}} \cdot f(t) \\
& =-N_{\mathrm{T}}\left(f_{\infty}+\left(f(0)-f_{\infty}\right)\right) \cdot \mathrm{e}^{-\left(e_{p}+e_{n}\right) t}
\end{aligned}
$$

berechnet werden kann ${ }^{[22]}$. Das Messsignal besteht somit aus einer Folge von Kapazitätstransienten mit temperaturabhängiger Zeitkonstante $\left(e_{p}+e_{n}\right)^{-1}$, wobei die Wiederholrate von der Wiederholfrequenz des Ladungspulses $f_{\mathrm{w}}$ abhängt. Zur Untersuchung auch kleiner Kapazitätsänderungen erfolgte in dieser Arbeit eine rechnerische Korrelation der Transienten vergleichbar der Korrelation mit Hilfe eines Lock-In-Verstärkers. Diese Art der Korrelation berechnet das Spektrum $S(T)$ aus der fundamentalen Fourierkomponen- 
te der Kapazitätstransienten:

$$
\begin{aligned}
S(T) & =f_{\mathrm{w}} \cdot \frac{2 N_{\mathrm{D} / \mathrm{A}}}{C_{0}} \int_{\frac{\varphi}{f_{\mathrm{w}}}}^{\frac{1}{f_{\mathrm{w}}}+\frac{\varphi}{f_{\mathrm{w}}}} \Delta C(t, T) \cdot \sin \left(2 \pi f_{\mathrm{w}} t-\varphi\right) \mathrm{d} t \quad \text { mit } \\
\varphi & =\frac{t_{\mathrm{p}}+t_{\mathrm{g}}}{2} f_{\mathrm{w}}
\end{aligned}
$$

Während des Beladungspulses und einer messtechnisch notwendigen Relaxationszeit $t_{\mathrm{g}}$ wird das zu korrelierende Signal auf Null gehalten. Die Phase der Korrelationsfunktion $\varphi$ ist so gewählt, dass ein konstantes $\Delta C$ nicht zu $S$ beiträgt. Im Linienmaximum des Spektrums eines Punktdefektes $\left(\frac{\mathrm{d} S}{\mathrm{~d} T}=0\right)$ gibt es unter der hier gemachten Annahme, dass der Besetzungsgrad innerhalb der gesamten Raumladungszone unabhängig vom Ort ist, einen festen, von der Art der Korrelationsfunktion abhängigen Zusammenhang zwischen dem Linienmaximum und der Dichte der umgeladenen Zustände $N_{\mathrm{T}}^{*}$ einerseits und zwischen $f_{\mathrm{w}}$ und $e_{n}(T)$ andererseits. So lässt sich durch geeignete Skalierung des Spektrums $N_{\mathrm{T}}^{*}$ im Maximum ablesen. Die Spektren, die in der Auswertung gezeigt sind, sind daher auf $\frac{2 \Delta C}{C_{0}} \cdot N_{\mathrm{D} / \mathrm{A}}$ skaliert (Die Ortsabhängigkeit des Besetzungsgrades wird in Anhang $\AA$ erläutert. Die hier gemachte Annahme bedeutet, dass $\frac{2 \Delta C}{C_{0}} \cdot N_{\mathrm{D} / \mathrm{A}}$ eine untere Grenze der tatsächlichen Dichte $N_{\mathrm{T}}^{*}$ ist.). Mit einem Modell von PONS ${ }^{[23]}$ kann die tatsächliche Dichte der Zustände $N_{\mathrm{T}}$ auch für den Fall, dass diese nicht klein im Vergleich zur Dichte der flachen Dotierung ist, berechnet werden.

Die Auswertung mehrerer, mit unterschiedlichen Frequenzen $f_{\mathrm{w}}$ gemessenen Spektren (Frequenzvariation) erlaubt mit Gleichung (2.4) durch die Arrhenius-Auftragung von $\ln \left(\frac{e_{n} / T^{2}}{\mathrm{~s}^{-1} \mathrm{~K}^{-2}}\right)$ gegen $1 / T$ die Bestimmung der Emissionskinetik, d.h. des zum beobachteten Zustands gehörenden Vorfaktors $\sigma_{n} \chi_{n}$ und deren Umladungsenthalpie $\Delta H_{n}$. Die Werte dieses Vorfaktors lassen sich demnach nicht aus den Eigenschaften der Emission separat bestimmen. Allerdings kann dazu unter Umständen die Einfangkinetik ausgenutzt werden, d.h. der Besetzungsgrad der tiefen Zustände in Abhängigkeit von der Dauer des Ladungspulses. Während der Einfangphase wird aus der Gl. (2.5) bei zu vernachlässigender Ladungsträgeremission im Falle ein n-dotierten Halbleiters $(p \approx 0)$ :

$$
f(t)=1+(f(0)-1) \cdot \mathrm{e}^{-c_{n} n \cdot t}
$$

Dieser Zusammenhang führt demzufolge zu einer exponentiellen Abhängigkeit der Besetzung $f$ von der Zeit $t$. Gemäß Gl. (2.7) ist die Kapazitätsänderung am Ende der Einfang- und zu Beginn der Emissionsphase

$$
\frac{2 \Delta C\left(t_{\mathrm{p}}\right)}{C_{0}} \cdot N_{\mathrm{D}}=-N_{\mathrm{T}} \cdot\left(1+(f(0)-1) \cdot \mathrm{e}^{-c_{n} n \cdot t_{\mathrm{p}}}\right)
$$


Ist der Vorfaktor $\sigma_{n} \chi_{n}$ aus der Emissionskinetik bestimmt, so lässt sich aus dem Zusammenhang Gl. (2.12) die Größe $c_{n} n$ und damit wegen Gl. (2.3) auch der Einfangquerschnitt $\sigma_{n}$ berechnen. Diese Analyse bedarf allerdings eine signifikante Differenz der entsprechenden Linienamplitude, die bei der minimalen und maximalen Einfangzeit $t_{\mathrm{p}}$ gemessen wird.

\subsubsection{DLTS an Vielelektronendefekten}

Bei räumlich ausgedehnten Defekten wie den Ausscheidungen, Versetzungskernzuständen oder Wolken von Punktdefekten mit hoher Dichte, etwa in der Umgebung von Versetzungen, können auf kleinem Raum viele umladbare Zustände existieren. Das Aufladen dieser Vielelektronenzustände ist mit dem Aufbau eines elektrostatischen Potentials $\Phi_{\mathrm{b}}$ verbunden, wobei $\Phi_{\mathrm{b}}$ von dem Besetzungsgrad $f$ der Defektzustände abhängt, wobei hier die lokale Näherung vorausgesetzt wird, d.h. die räumliche Ausdehnung der Defektzustandsdichte ist vernachlässigbar im Vergleich zur Ausdehnung des Potentials:

$$
-e \Phi_{\mathrm{b}}=E_{\mathrm{b}} f
$$

Hier ist $E_{\mathrm{b}}$ die elektrostatische Energie (Abb. 2.2). Dieser Zusammenhang wurde von

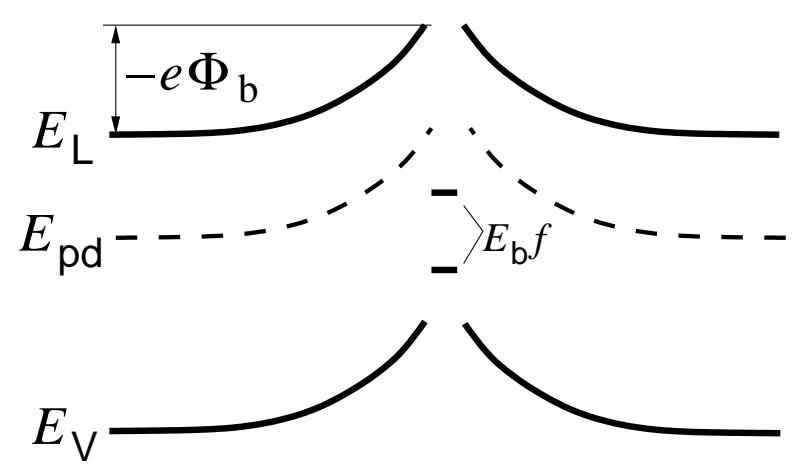

Abbildung 2.2 - Bandverbiegung durch die Beladung eines Vielelektronendefektes. $\Phi_{\mathrm{b}}$ ist das Potential aufgrund der Besetzung des Vielelektronendefektes mit dem Besetzungsgrad $f$.

SCHRÖTER UND LABUSCH ${ }^{[24]}$ für Versetzungskerndefekte und von SHIKIN UND ShIKI$\mathrm{NA}^{[25]}$ für Punktdefekte im Verzerrungsfeld von Versetzungen berechnet.

Die Zustandsdichte eines Vielelektronendefektes resultiert aus der Überlagerung der Zustandsdichten der beteiligten einzelnen Defekte. Ob sich die Vielelektronendefektzustandsdichte als eine Summe diskreter Zustandsdichten oder als kontinuierliche Zustandsdichte beschreiben lässt, ergibt sich aus der Rate, mit der die einzelnen Defektzustände Ladungen untereinander austauschen können. Dabei werden zwei Extremfälle 
unterschieden, die als Modell A und Modell B beschrieben werden ${ }^{[26]}$. Im Modell A ist die Rate des Ladungsaustausches innerhalb der Defektzustände vernachlässigbar verglichen mit dem Ladungsaustausch mit dem Valenz- bzw. Leitungsband. Dann liegen die Defekte offensichtlich nahe genug, um ein gemeinsames elektrostatisches Potential $\Phi_{\mathrm{b}}$ zu bilden, jedoch weit genug voneinander entfernt, um Ladungsträger austauschen zu können. Defekte dieser Art werden als ausgedehnte Defekte mit lokalisierten Zuständen bezeichnet. Im Modell B ist der direkte Ladungsaustausch untereinander schnell im Vergleich zum Austausch mit dem Valenz- bzw. Leitungsband. In diesem Fall stellen sich die Besetzungsgrade der einzelnen Niveaus gemäß einer Quasifermiverteilung ein, die im Nichtgleichgewichtsfall nicht mit dem Fermi-Niveau des Systems aus Valenzund Leitungsband übereinstimmen muss. Diese Defekte werden als ausgedehnte Defekte mit bandartigen Zuständen bezeichnet. Die folgende Beschreibung beschränkt sich auf die Konsequenzen aus dem Modell A. Detaillierte Betrachtungen finden sich bei HedEMANN [26].

In der Nähe ausgedehnter Defekte ist die Dichte der freien Ladungsträger aufgrund des Potentials $\Phi_{\mathrm{b}}$ gemäß $n \cdot \exp \left(\frac{-E_{\mathrm{b}} f}{k_{\mathrm{B}} T}\right)$ modifiziert. Dementsprechend ändert sich die Ratengleichung (2.2) $\mathrm{zu}$

$$
\frac{\mathrm{d} f}{\mathrm{~d} t}=c_{n} n \cdot \exp \left(\frac{-E_{\mathrm{b}} f}{k_{\mathrm{B}} T}\right)(1-f)-e_{n} f
$$

wobei hier angenommen ist, dass der Defekt einen diskreten Zustand erzeugt, dessen Ladungsträgereinfang von der Einfangbarriere $E_{\mathrm{b}}$ beeinflusst wird. In diesem Fall liegt, anders als bei Gl. (2.11), in erster Näherung ein logarithmischer Zusammenhang zwischen der Besetzung $f$ und der Zeit $t$ vor $\underline{[26]}$. Dieser Fall eines Punktdefektes mit Einfangbarriere wird von QUEISSER ${ }^{[27]}$ ausführlich analysiert.

Für den Fall, dass ein Spektrum von $d$ diskreten Energieniveaus vorliegt, ergibt sich die Veränderung der Besetzung aus einem System von Differentialgleichungen, die durch den Gesamtbesetzungsgrad $F$ gekoppelt sind:

$$
\frac{\mathrm{d} f_{i}}{\mathrm{~d} t}=c_{i, n} n \cdot \exp \left(\frac{-E_{\mathrm{b}} F}{k_{\mathrm{B}} T}\right)\left(1-f_{i}\right)-e_{i, n} f_{i}, \quad i=1 \ldots d
$$

Hier ist $f_{i}$ der Besetzungsgrad des $i$-ten Energieniveaus. Der Gesamtbesetzungsgrad $F$ ergibt sich aus der mit ihren Zustands Im Falle ausgedehnter Defekte mit bandartigen Zuständen steigen die absoluten Höhen der Maxima in der Pulslängenvariation kaum an, während sich das Maximum auf der Temperaturskala verschiebt [28]. Diese Einfangkinetiken erlauben demnach qualitative Rückschlüsse auf die lokale Dichte der Vielelektronenzustände. Da es sich in beiden Fällen in der Regel um eine Verteilung von Zuständen und nicht, wie bei Punktdefekten, um einen diskreten Zustand in der Bandlücke handelt, 
ist die oben beschriebene Messung der Emissionskinetik kein Mittel, um Aussagen über die energetische Lage in der Bandlücke zu treffen.

\subsubsection{Modell zur Beschreibung von DLTS-Spektren ausgedehnter Vielelektronendefekte}

Wie im vorigen Abschnitt beschrieben, bietet die Emissionskinetik im Falle eines Punktdefektes Informationen über dessen Aktivierungsenthalpie und den Faktor aus Einfangquerschnitt und Entropiefaktor $\sigma_{n} \chi_{n}$. Für ausgedehnte Defekte liefert die Einfangkinetik qualitative Aussagen über lokale Dichten der Vielelektronenzustände. Sollen der Einfangquerschnitt und der Entropiefaktor von Punktdefekten separat bestimmt oder Informationen über die den Vielelektronenzuständen zugrunde liegenden Zustandsdichten und Konzentrationen gewonnen werden, so ist es notwendig, diese Parameter durch Anpassung eines Modells an die gemessenen Spektren zu bestimmen. Dazu werden in beiden Fällen Datensätze aus der Frequenz- und der Pulslängenvariation benötigt.

Das dem Einfang und der Emission von Ladungsträgern an den Defektzuständen während der DLTS zugrunde liegende Modell, das neben dem sogenannten Einfang aus dem Debye-Tail (siehe Anhang A) auch den Ladungsaustausch mit beiden Bändern berücksichtigt (anders als in Gl. (2.15)), ist ein System von Ratengleichungen (vgl. Gl.(2.15)):

$$
\frac{\mathrm{d} f_{i}}{\mathrm{~d} t}=R_{i, n}^{\mathrm{cap}}+R_{i, n}^{\mathrm{em}}+R_{i, p}^{\mathrm{cap}}+R_{i, p}^{\mathrm{em}}, \quad i=1 \ldots d
$$

Hier beschreiben die $R$ die besetzungsabhängigen Einfang- und Emissionsraten (Indizes cap bzw. ${ }^{\mathrm{em}}$ ) der Elektronen und Löcher (Indizes $n$ bzw. $p$ ):

$$
\begin{aligned}
R_{i, n}^{\mathrm{cap}} & =\sigma_{i, n}\left\langle v_{\mathrm{th}}\right\rangle n\left(1-f_{i}\right) \cdot \exp \left(-\frac{E_{\mathrm{b}} F}{k_{\mathrm{B}} T}\right) \\
R_{i, n}^{\mathrm{em}} & =\sigma_{i, n}\left\langle v_{\mathrm{th}}\right\rangle N_{\mathrm{L}} f_{i} \chi_{i, n} \cdot \exp \left(-\frac{\Delta H_{i}}{k_{\mathrm{B}} T}\right) \\
R_{i, p}^{\mathrm{cap}} & =\sigma_{i, p}\left\langle v_{\mathrm{th}}\right\rangle p f_{i} \cdot \exp \left(-\frac{E_{\mathrm{b}} F}{k_{\mathrm{B}} T}\right) \\
R_{i, p}^{\mathrm{em}} & =\sigma_{i, p}\left\langle v_{\mathrm{th}}\right\rangle N_{\mathrm{V}}\left(1-f_{i}\right) \chi_{i, p} \cdot \exp \left(-\frac{E_{\mathrm{g}}-\Delta H_{i}}{k_{\mathrm{B}} T}\right)
\end{aligned}
$$

$\left\langle v_{\mathrm{th}}\right\rangle$ steht für die mittlere thermische Geschwindigkeit und $N_{\mathrm{L} / \mathrm{V}}$ für die effektiven Zustandsdichten im Leitungs-/Valenzband sowie $E_{\mathrm{g}}$ für die Bandlückenenergie. Die Dichte der Minoritätsladungsträger berechnet sich nach [29]

$$
p(x)=n_{\mathrm{i}} \exp \left(-\frac{\Phi_{\mathrm{SB}}(x)}{k_{\mathrm{B}} T}\right),
$$


wobei $n_{\mathrm{i}}$ die intrinsische Ladungsträgerdichte und $\Phi_{\mathrm{SB}}(x)$ den Potentialverlauf innerhalb der Raumladungszone darstellen. Da $n_{\mathrm{i}} \ll N_{\mathrm{L}}, N_{\mathrm{V}}$ während der Emissionsphase und $n_{\mathrm{i}} \ll n$ während der Einfangphase ist, liefert der Term der Einfangrate der Minoritätsladungsträger (hier $R_{\mathrm{i}, \mathrm{p}}^{\mathrm{cap}}$ ) keinen Beitrag zur Umladung tiefer Zustände während der DLTS.

Ist der zu beschreibende Defekt ein Punktdefekt ohne Einfangbarriere, so ist $d=1$ und $E_{\mathrm{b}}=0$ und Gl.(2.16) reduziert sich zu Gl.(2.2).

\subsection{Gold in Silizium}

In diesem Abschnitt werden die bekannten Eigenschaften von Gold in Silizium zusammengefasst. Zunächst werden die Diffusions- und Löslichkeitseigenschaften im Abschnitt 2.2 .1 beschrieben. Im Anschluss gibt Abschnitt 2.2.2 einen kurzen Überblick über die Kenntnisse der Bildung von Goldausscheidungen. Schließlich werden im Abschnitt 2.2.3 die elektrischen Eigenschaften der tiefen Zustände des Goldes erörtert.

\subsubsection{Diffusion und Löslichkeit}

Gold besetzt in Silizium sowohl interstitielle als auch substitutionelle Gitterplätze. Während die Löslichkeit der substitutionellen Spezies größer ist als die der interstitiellen, verläuft die Diffusion fast ausschließlich über Zwischengitterplätze ${ }^{[30]}$. Es konnte gezeigt werden, dass der Austausch zwischen interstitiellen und substitutionellen Gitterplätzen für Temperaturen von $800^{\circ} \mathrm{C}$ und höher hauptsächlich über den sogenannten „kick-out“Mechanismus

$$
\mathrm{Au}_{\mathrm{i}} \rightleftharpoons \mathrm{Au}_{\mathrm{s}}+\mathrm{I}
$$

stattfindet $\underline{\underline{311}}$. Hier bezeichnen $\mathrm{Au}_{\mathrm{i}}$ die interstitiellen, $\mathrm{Au}_{\mathrm{s}}$ die substitutionellen Goldatome und I die Siliziumzwischengitteratome (im folgenden nur Zwischengitteratome). Mit der Eindiffusion von Gold und der Reaktion (2.21) ist eine Übersättigung von Zwischengitteratomen verbunden. In versetzungsfreiem Silizium wirken ausschließlich die Oberflächen als Senke zum Abbau dieser Übersättigung. Somit wird die Anreicherung von Gold durch die Ausdiffusion der Zwischengitteratome limitiert. Es bildet sich ein U-förmiges Goldkonzentrationsprofil aus, dessen Form sich mit der Gleichgewichtskonzentration $C_{\mathrm{I}}^{\mathrm{eq}}$, dem Diffusionskoeffizienten $D_{\mathrm{I}}$ der Zwischengitteratome und den Gleichgewichtskonzentrationen des interstitiellen und substitutionellen Goldes, $C_{\mathrm{i}}^{\mathrm{eq}}, C_{\mathrm{s}}^{\mathrm{eq}}$, durch einen effektiven Diffusionskoeffizienten

$$
D_{\mathrm{Au}}^{\mathrm{I}, *}=\frac{C_{\mathrm{I}}^{\mathrm{eq}}}{C_{\mathrm{i}}^{\mathrm{eq}}+C_{\mathrm{s}}^{\mathrm{eq}}} D_{\mathrm{I}}
$$

beschreiben lässt $\underline{[32.33]}$. 
In versetzungshaltigem Silizium hingegen können die in Übersättigung vorliegenden Zwischengitteratome an den Versetzungen beseitigt werden. Dies führt zu einer größeren effektiven Golddiffusion. Erreicht die Versetzungsdichte einen kritischen Wert, der durch Pichaud Und Mitarbeiter ${ }^{[34]}$ für Silizium auf $10^{7} \mathrm{~cm}^{-2}$ bestimmt wurde, so verläuft der Abbau der Übersättigung so schnell, dass die Eindiffusion interstitieller Goldatome (Diffusionskoeffizient $D_{\mathrm{i}}$ ) ratenlimitierend für die kick-out-Reaktion wird. Der effektive Diffusionskoeffizient berechnet sich dann nach

$$
D_{\mathrm{Au}}^{\mathrm{i}, *}=\frac{C_{\mathrm{i}}^{\mathrm{eq}}}{C_{\mathrm{i}}^{\mathrm{eq}}+C_{\mathrm{s}}^{\mathrm{eq}}} D_{\mathrm{i}}
$$

In diesem Fall besitzt das Konzentrationsprofil $C_{\mathrm{s}}(x, t)$ der substitutionellen Spezies die Form

$$
\frac{C_{\mathrm{s}}(x, t)}{C_{\mathrm{s}}^{\mathrm{eq}}}=\operatorname{erfc}\left(\frac{x}{2 \sqrt{D_{\mathrm{Au}}^{\mathrm{i}, *} t}}\right),
$$

wobei in diesem Modell eine Segregation von Gold an Versetzungen unberücksichtigt bleibt. Die Diffusionsprofile, auf deren Grundlage StolwiJk und Mitarbeiter ${ }^{[30]}$ dieses Modell erstellt haben, wurden mit der Ausbreitungswiderstandstechnik (,Spreading Resistance Technique" ${ }^{[35]}$ bestimmt. Bei dieser Methode werden lokale kompensatorische Effekte des Goldes auf die Leitfähigkeit gemessen, die ohne den Einfang von Ladungsträgern an den Goldatomen durch die flache Dotierung gegeben ist.

Im oberflächennahen Bereichen wurde die Diskrepanz zwischen gemessenen und auf Grundlage von Gl. (2.21) berechneten Profilen dadurch erklärt, dass aufgrund der hier merklichen Eindiffusion von Leerstellen von der Oberfläche ein weiterer Mechanismus des Einbaus von Goldatomen in das Kristallgitter berücksichtigt werden muss ${ }_{\text {[36] }}$. In diesem sogenannten „Frank-Turnbull“-Mechanismus nimmt ein interstitielles Fremdatom den Platz einer Leerstelle ein.

RoDRIGUEZ UND MitARBEITER ${ }^{[20]}$ finden durch die Neutronenaktivierungsanalyse von versetzungshaltigen Proben Goldkonzentrationensprofile, die sich über einen großen Probenbereich durch Fehlerfunktionsprofile anpassen lassen. Die dabei bestimmten effektiven Diffusionskoeffizienten stimmen dort nur für Temperaturen über $1000^{\circ} \mathrm{C}$ mit dem Modell (2.23) überein. Auf der Basis der Reaktion von interstitiellem Gold mit Bindungsplätzen im Versetzungskern T

$$
\mathrm{Au}_{\mathrm{i}}+\mathrm{T} \rightleftharpoons \mathrm{Au}_{\mathrm{T}}+\mathrm{E}
$$

$\mathrm{zu}$ Gold $\mathrm{Au}_{\mathrm{T}}$, das im Versetzungskern gebunden ist und einem leeren interstitiellen Gitterplatz E, die zusätzlich zum kick-out-Mechanismus (2.21) auftritt, wird dort ein effektiver Diffusionskoeffizient $D_{\mathrm{Au}}^{\mathrm{T}, *}$ hergeleitet, der den Einfang interstitiellen Goldes 
im Kern der Versetzungen berücksichtigt:

$$
D_{\mathrm{Au}}^{\mathrm{T}, *}=\frac{C_{\mathrm{i}}^{\mathrm{eq}} D_{\mathrm{i}}}{C_{\mathrm{i}}^{\mathrm{eq}}+C_{\mathrm{s}}^{\mathrm{eq}}+C_{\mathrm{Au}}^{\mathrm{eq}}}
$$

Dabei stellt $C_{\mathrm{Au}_{\mathrm{T}}}^{\mathrm{eq}}$ die Gleichgewichtskonzentration von Gold im Versetzungskern dar. Zugrunde gelegt wurde hier, dass der Besetzungsgrad der Bindungsplätze klein ist $\left(C_{\mathrm{Au}_{\mathrm{T}}}^{\mathrm{eq}} \ll\right.$ $C_{\mathrm{T}}^{\mathrm{eq}}+C_{\mathrm{AuT}}^{\mathrm{eq}}, C_{\mathrm{T}}^{\mathrm{eq}}$ : Gleichgewichtskonzentration der unbesetzten Bindungsplätze). Das Verhältnis dieser Gleichgewichtskonzentration und der des substitutionellen Goldes $C_{\mathrm{s}}^{\mathrm{eq}}$ wird in der Näherung $C_{\mathrm{i}}^{\mathrm{eq}} \ll C_{\mathrm{s}}^{\mathrm{eq}}$ durch einen Segregationskoeffizienten

$$
S=\frac{C_{\mathrm{Au}_{\mathrm{T}}}^{\mathrm{eq}}}{C_{\mathrm{s}}^{\mathrm{eq}}} \frac{V}{V_{\mathrm{d}}} \approx\left(\frac{D_{\mathrm{Au}}^{\mathrm{i}, *}}{D_{\mathrm{Au}}^{\mathrm{T}, *}}-1\right) \frac{V}{V_{\mathrm{d}}}
$$

beschrieben, wobei $V$ das Kristallvolumen und $V_{\mathrm{d}}=\pi r_{d}^{2} N_{\mathrm{d}} V$ das Volumen der zylinderförmigen Versetzungen mit dem Radius $r_{\mathrm{d}}$ und der Versetzungsdichte $N_{\mathrm{d}}$ bezeichnen. Damit wird die Diffusion folgendermaßen beschrieben:

$$
\begin{aligned}
D_{\mathrm{Au}}^{\mathrm{T}, *} & =\frac{D_{\mathrm{Au}}^{\mathrm{i}, *}}{\epsilon S+1}, \text { mit } \\
\epsilon & =\pi r_{\mathrm{d}}^{2} N_{\mathrm{d}}
\end{aligned}
$$

Die Anpassung des Segregationskoeffizienten an die gemessenen Daten liefert eine Bindungsenergie von $2.72(46) \mathrm{eV}$.

In der Arbeit von Poisson und Mitarbeiter ${ }^{[37]}$ werden Konzentrationsprofile des radioaktiv markierten Goldes ${ }^{195} \mathrm{Au}$ in polykristallinem Silizium mit unterschiedlichen Korngrößen und Versetzungsdichten gemessen. Für Diffusionstemperaturen über $897^{\circ} \mathrm{C}$ ist es möglich, die gemessenen Profile durch Gl.(2.24) anzupassen. Die Profile für tiefere Diffusionstemperaturen können in zwei Abschnitte aufgeteilt werden: Der oberflächennahe Bereich entspricht ebenfalls dem durch Gl.(2.24) beschriebenen Verhalten, während die Konzentration im oberflächenfernen Bereich exponentiell abfällt (Abb. 2.3) .

Erklärt wird dieser exponentielle Abfall mit einer Segregation von Goldatomen an den Korngrenzen. Durch Anpassung eines effektiven Diffusionskoeffizienten gemäß Gl.(2.28) wurde eine Bindungsenergie von $1.46 \mathrm{eV}$ berechnet.

\subsubsection{Ausscheidungen}

Metallausscheidungen in Silizium können sich bilden, wenn die vollständig gelöste metallische Verunreinigung in Übersättigung gerät, d.h. wenn die Konzentration des Metalls im Silizium höher ist, als die Konzentration im Gleichgewicht mit der benachbarten Pha- 


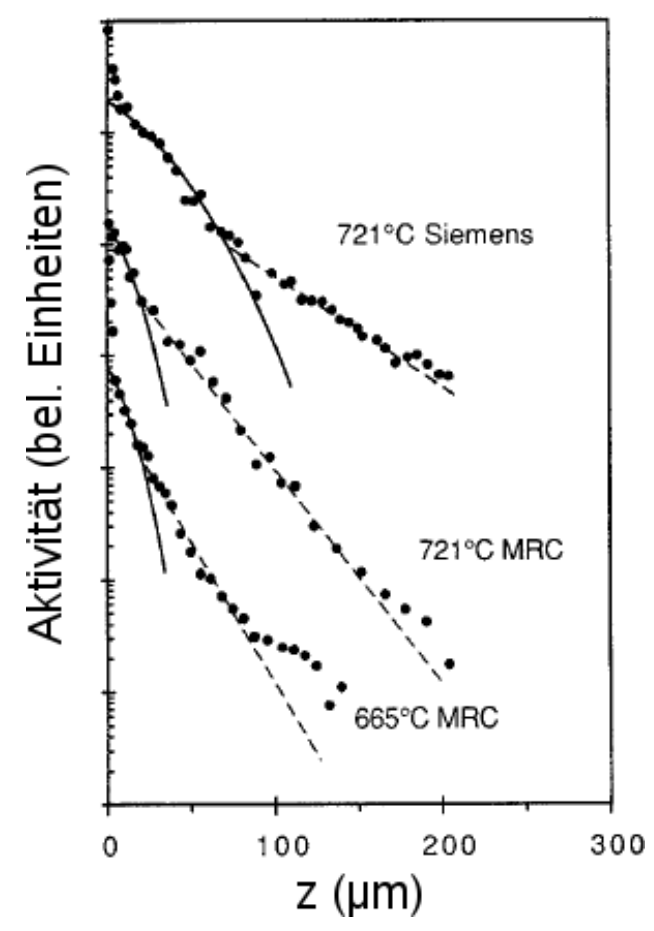

Abbildung 2.3 - Das Profil der zur Goldkonzentration proportionalen Aktivität folgt in Oberfächennähe einer erfc-Funktion (durchgezogene Linie). In den oberflächenferneren Bereichen nimmt die Konzentration exponentiell ab (gestrichelte Linie). Es wurden unterschiedliche Ausgangsmaterialien benutzt $\underline{[37]}$.

se im Phasendiagramm, wodurch die Löslichkeit definiert ist. Bei Diffusionsexperimenten mit Gold ist diese benachbarte Phase eine flüssige Phase, die sich üblicherweise auf der Oberfläche befindet. Diese stellt sich bei üblichen Diffusionstemperaturen, die oberhalb der eutektischen Temperatur von $370^{\circ} \mathrm{C}$ liegen, ein. Übersättigung entsteht während der Abkühlung von Temperaturen, bei denen die Konzentration ins Gleichgewicht gebracht wurde. Ob und wie sich die Metallatome während dieser Abkühlung ausscheiden, hängt wesentlich von der Abkühlrate, der Dichte der Keimbildungsplätze und der Diffusion der Metallatome ab. Im Gegensatz zu anderen Übergangsmetallen wie z.B. Eisen, Nickel oder Kupfer, die in Silizium sehr schnell diffundieren und deren Ausscheidungsbildung sich nur schwer oder gar nicht verhindern lässt, scheidet sich Gold wegen der langsamen effektiven Diffusion nur sehr schwer aus. BAUMANN UND SCHRÖTER ${ }^{[38]}$ haben die Bildung von Ausscheidungen in stark mit Gold übersättigten und dann unterschiedlich lang ausgelagerten Siliziumproben untersucht. Mittels hochauflösender Transmissionselektronenmikroskopie (HRTEM) wurden in den Proben sowohl extrinsische Stapelfehler als auch sphärische Goldsilizidausscheidungen mit orthorhombischer Struktur gefunden, deren Keimbildung und Wachstum korreliert waren. Da gemäß des eutektischen Phasendiagramms des binären Systems Gold/Silizium im thermodynamischen Gleichgewicht 
keine Goldsilizide existieren, waren diese dort gefundenen Ausscheidungen metastabil.

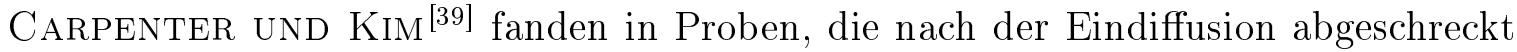
worden waren, polykristalline Goldausscheidungen mit kubisch-flächenzentrierter Struktur. Die Entstehung wird durch die während des Abschreckens in Übersättigung geratenen Goldatome erklärt. In anderen Proben, die mit Versetzungen in der Nähe der Goldschicht versehen waren, wurden Ausscheidungen an solchen Versetzungsreihen gefunden, die mit der goldbedeckten Oberfläche verbunden waren. Dieser Umstand wurde mit dem Effekt der beschleunigten Diffusion entlang von Versetzungen (,pipe diffusion" “부] in Zusammenhang gebracht.

Auch Rodriguez und Mitarbeiter [20] fanden mit Transmissionselektronenmikroskopie in versetzungshaltigen Siliziumproben sphärische Goldausscheidungen in der Nähe der Oberfläche. Auch hier wurde die Eindiffusion durch Abschrecken beendet.

\subsubsection{Tiefe Zustände}

In Silizium gelöstes substitutionelles Gold besitzt zwei tiefe Zustände: einen Akzeptorund einen Donatorzustand $\left(\mathrm{Au}^{(0 /-)}, \mathrm{Au}^{(+/ 0)}\right)$. Ein Überblick über Größen der Aktivierungsenthalpien und Einfangquerschnitte dieser tiefen Zustände findet sich bei SCHRÖTER UND SEIBT ${ }^{[41]}$. Die Werte für die Aktivierungsenthalpie des Akzeptorzustandes, d.h. die Energiedifferenz des Zustandes zum Leitungsband, liegen demnach zwischen $0.533 \mathrm{eV}$ und $0.56 \mathrm{eV}$. Der Einfangquerschnitt dieses Defektes nimmt Werte zwischen $8.5 \cdot 10^{-17} \mathrm{~cm}^{2}$ und $2 \cdot 10^{-16} \mathrm{~cm}^{2}$ an. Die Aktivierungsenthalpie des Donators (Energiedifferenz zwischen Donatorzustand und Valenzband) hat einen Wert zwischen $0.31 \mathrm{eV}$ und $0.346 \mathrm{eV}$. Dessen Einfangquerschnitt liegt zwischen $1.1 \cdot 10^{15} \mathrm{~cm}^{2}$ und $1.1 \cdot 10^{14} \mathrm{~cm}^{2}$.

SveINBJÖRNSSON UND ENGSTRÖM ${ }^{[42]}$ haben den Einfluss von Wasserstoff auf das Spektrum tiefer Zustände von golddotierten Siliziumproben mittels DLTS (siehe Abschnitt 2.1.1) untersucht. Neben den DLTS-Linien der oben erwähnten goldspezifischen tiefen Zustände wurden zusätzliche Linien (G1, G2, G3, G4) gefunden, deren Amplituden von der in der Raumladungszone verfügbaren Wasserstoffkonzentration abhing. Die Linien G1, G2 und G4 gehören demnach zu einem Zustand eines Gold-WasserstoffKomplexes (AuH), wobei G2 dem Donator-, G4 dem Akzeptor- und G1 dem Doppelakzeptorniveau $\left(\mathrm{AuH}^{(+/ 0)}, \mathrm{AuH}^{(0 /-)}, \mathrm{AuH}^{(-/-)}\right)$dieses Zustands zugeordnet werden. Die Herkunft der G3-Linie wird einem wasserstoffbedingten Defekt, jedoch nicht dem AuHKomplex zugeordnet. Die Emissionseigenschaften von $\mathrm{AuH}^{(0 /-)}$ sind denen des $\mathrm{Au}^{(0 /-)}$ Niveaus ähnlich, d.h. in der konventionellen DLTS lassen sich die Linien dieser beider Niveaus nicht unterscheiden. In $\underline{[42]}$ konnte die Existenz von $\mathrm{AuH}^{(0 /-)}$ unter anderem dadurch nachgewiesen werden, dass die AuH-Komplexe durch Auslagern für eine halbe Stunde bei $250^{\circ} \mathrm{C}$ dissoziiert wurden. Die anschließend gemessenen DLTS-Spektren zeigten eine signifikante Reduktion der AuH-induzierten Linienamplituden und einen Anstieg sowie eine Temperaturverschiebung der Linie, die vor dem Auslagern dem $\mathrm{Au}^{(0 /-)}$ und dem $\mathrm{AuH}^{(0 /-)}$ zugeordnet werden konnte. 
Mit der hochauflösenden Laplace-DLTS-Technik konnten die Emissionseigenschaften dieser Defekte separat ermittelt werden [43]. Durch die Messung der Emissionskinetik (siehe Abschnitt 2.1.1) wurden die Aktivierungsenthalpien von $\mathrm{AuH}^{(0 /-)} \mathrm{zu} 0.542 \pm 8 \mathrm{eV}$ und von $\mathrm{Au}^{(0 /-)} \mathrm{zu} 0.578 \pm 1 \mathrm{eV}$ bestimmt. Das Verhältnis der Einfangquerschitte betrug $\sigma_{n}^{\mathrm{AuH}^{(0 /-)}}=0.6 \sigma_{n}^{\mathrm{Au}^{(0 /-)}}$.

\subsection{Versetzungen in Silizium}

Für die Wechselwirkungen metallischer Verunreinigungen mit Versetzungen sind unterschiedliche Versetzungseigenschaften maßgeblich. Dieser Abschnitt behandelt die für die Ergebnisse dieser Arbeit wichtigen Eigenschaften von Versetzungen in Silizium. Zunächst werden strukturelle Eigenschaften von Stufenversetzungen beschrieben (Abschnitt 2.3.1). Abschnitt 2.3.2 erläutert die elektrischen Eigenschaften.

\subsubsection{Strukturelle Eigenschaften}

\subsubsection{Das Gleitsystem im Siliziumkristall}

Die Siliziumkristallstruktur ist ein kubisch-flächenzentriertes Bravais-Gitter mit zweiatomiger Basis (Diamantgitter). Die Gleitsysteme dieses Kristalls sind vom Typ $\{111\}$, $\langle 110\rangle$. Aus der zweiatomigen Basis resultieren zwei unterschiedliche Typen von Gleitebenen, die durch ihren Abstand und der Anzahl der aufzubrechenden Bindungen beim Abgleiten charakterisiert werden: das sogenannte „Glide-Set" und das „Shuffle-Set" $\underline{\text { [44] }}$. Der Abstand zwischen den Ebenen des Glide-Sets beträgt ein Drittel des Ebenenabstandes im Shuffle-Set. Die Anzahl der aufzubrechenden Bindungen im Glide-Set ist das Dreifache der Anzahl beim Shuffle-Set. Trotz der somit zu erwartenden kleineren Scherspannung, die beim Abgleiten im Shuffle-Set aufgewendet werden muss, ist die vorherrschende Meinung, dass unter üblichen Verformungsbedingungen das Glide-Set

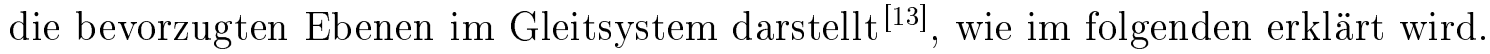

Die Linien der minimalen Peierlspotentiale (Peierlstäler) liegen im Diamantgitter entlang der [110]-Richtungen. Somit nimmt der Burgersvektor einer geradlinigen Versetzung entweder einen $60^{\circ}$ - oder einen $0^{\circ}$-Winkel (Schraubenversetzung) zur Versetzungslinie ein. Der Burgersvektor einer idealen $60^{\circ}$-Versetzung ist vom Typ $\frac{a}{2}\langle 110\rangle$ (a: Gitterkonstante). Im Gegensatz zum Shuffle-Set kann diese Versetzung im Glide-Set in zwei ShockleyPartialversetzungen dissoziieren: $\frac{a}{2}[01 \overline{1}]=\frac{a}{6}[11 \overline{2}]+\frac{a}{6}[\overline{1} 2 \overline{1}]$. Dabei spannen diese $30^{\circ}$ und $90^{\circ}$-Partialversetzungen einen Stapelfehler auf. Diese Dissoziation konnte mit der „weak-beam-Technik“ der Transmissionselektronenmikroskopie gezeigt werden ${ }^{\text {[45] }}$. Darüber hinaus unterscheiden sich die Versetzungen der beiden Systeme in ihrer Konfiguration ungesättigter Bindungen („dangling bonds“) des Versetzungskerns. Theoretische Untersuchungen konnten zeigen, dass die Konfiguration des Glide-Sets eine Rekonstruktion der ungesättigten Bindungen im Versetzungskern ermöglicht ${ }^{[44]}$. Die Neigung, elas- 
tische Spannungen durch Abgleiten über das Glide-Set abzubauen, resultiert somit aus der geringeren elastischen Energie und der kleineren Energie ungesättigter Bindungen. Durch die Verformung bei Temperaturen bis $150^{\circ} \mathrm{C}$ mit einer Belastung von $5 \mathrm{GPa}$ konnten RABIER Und Demenet $\underline{[46]}$ Versetzungen im Shuffle-Set erzeugen.

\subsubsection{Das elastische Verzerrungsfeld}

Durch die Verzerrung des Gitters erzeugen Versetzungen langreichweitige interne Spannungen, deren hydrostatische Komponente im Falle einer Stufenversetzung eine Funktion von $\frac{\sin \Theta}{r}$ ist $\underline{47]}$. Abb. 2.4 zeigt die daraus resultierenden Linien konstanten hydrostatischen Drucks im Dilatationsfeld einer Stufenversetzung. Dabei sind $\Theta$ und $r$ die Po-

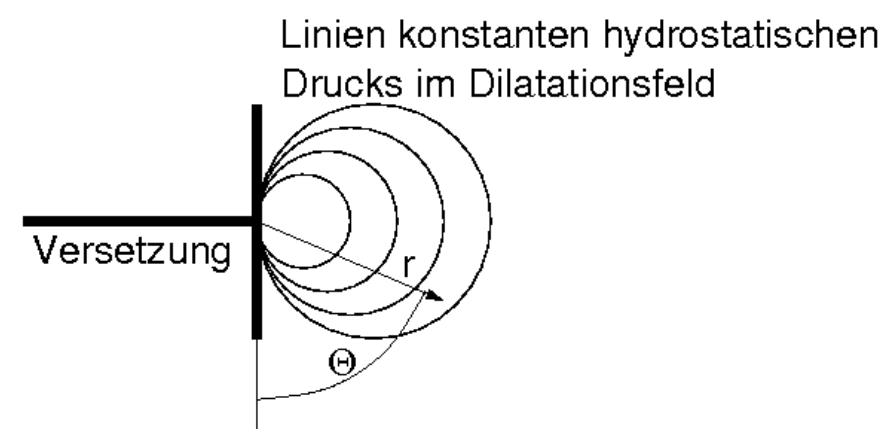

Abbildung 2.4 - Das Verzerrungsfeld einer Stufenversetzung, die Kreise markieren Linien konstanten hydrostatischen Drucks im Dilatationsfeld ( $r$, $\Theta$ : Polarkoordinaten).

larkoordinaten mit dem Versetzungskern als Ursprung. Da sich in der Gleitebene der Versetzung das Vorzeichen des hydrostatischen Drucks ändert, liegt dort ein zum Dilatationsfeld symmetrisches Kompressionsfeld vor. Fremdatome wechselwirken mit dem hydrostatischen Druck des Verzerrungsfeldes aufgrund des Größenunterschieds ihrer kovalenten Radien und der Radien der Atome des Wirtsgitters. Auf diese Wechselwirkung zwischen Versetzungen und Fremdatomen wird im Kap. 2.4.1 näher eingegangen.

\subsubsection{Elektrische Eigenschaften}

Theoretischen Berechnungen zufolge besitzen ideale Versetzungen rekonstruierte Versetzungskerne und keine tiefen Zustände in der Bandlücke늘. Die tiefen Zustände der Versetzungen rühren von Kerndefekten, wie den Rekonstruktionsdefekten, Kinken und Sprüngen und von Punktdefekten her, die im Kern oder im Verzerrungsfeld gebunden

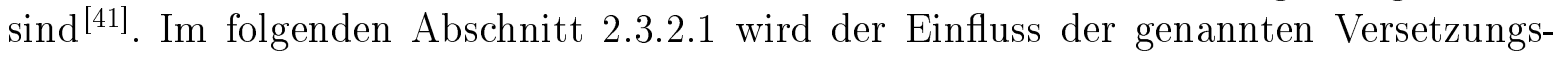
kerndefekte auf die Bandstruktur beschrieben. Im darauf folgenden Abschnitt 2.3.2.2 werden die intrinsischen elektrischen Eigenschaften des Verzerrungsfeldes und die der dort gebundenen Punktdefekte erläutert. 


\subsubsection{Effekt der Linienladung}

Geladenen Defektzustände an Versetzungen erzeugen eine Linienladung entlang der Versetzungen, die in der Literatur auf unterschiedliche Weise beschrieben werden. SCHRÖTER UND LABUSCH ${ }^{[24]}$ beschreiben die diskreten Ladungen entlang des Versetzungskerns durch ein im neutralen Zustand halb gefülltes eindimensionales Band. Der Besetzungsgrad $f_{\mathrm{V}}$ nimmt dann Werte zwischen -1 und 1 an je nachdem, ob das Band Elektronen abgegeben $\left(-1<f_{\mathrm{V}}<0\right)$ oder aufgenommen $\left(0<f_{\mathrm{V}}<1\right)$ hat. Dabei wird die Linienladung $\frac{-e f_{\mathrm{V}}}{d}$ entlang der Versetzung durch ein langreichweitiges elektrisches Feld abgeschirmt. Hier sind $e$ die Elementarladung und $d$ der Abstand der amphoteren Defekte im Versetzungskern. Unter der Annahme, dass die Defekte im neutralen Zustand die Energie $E_{\mathrm{V}}^{(0)}$ besitzen, und die Linienladung ab einem Radius $r_{0}$ als kontinuierlich angesehen werden kann, beträgt die Energie der geladenen Versetzung für kleine Besetzungsgrade $\left(\left|f_{\text {dis }}\right| \ll 1\right)^{[13]}$

$$
\begin{aligned}
E_{\mathrm{V}, \mathrm{SL}}\left(f_{\mathrm{V}}\right) & =E_{\mathrm{V}}^{(0)}+\frac{e^{2} f_{\mathrm{V}}}{2 \pi \epsilon \epsilon_{0} d}\left(\ln \left(\frac{\lambda}{r_{0}}\right)+\frac{1}{4} \frac{N_{\mathrm{D}}-N_{\mathrm{A}}}{n-p}+\frac{1}{4}\right) \mathrm{mit} \\
\lambda & =\sqrt{\frac{\epsilon \epsilon_{0} k_{\mathrm{B}} T}{e^{2}(n+p)}} \text { (Debye'sche Abschirmlänge). }
\end{aligned}
$$

Hier bezeichnen $\epsilon$ und $\epsilon_{0}$ die Permittivitätszahl bzw. die elektrische Feldkonstante, $n$ und $p$ die Elektronen- bzw. Löcherdichte sowie $N_{\mathrm{D}}$ und $N_{\mathrm{A}}$ die Donator- und Akzeptordichte. READ ${ }^{[48]}$ beschreibt die Ladungen entlang der Versetzungen als Reihe diskreter Akzeptoren mit einem Abstand $d / f_{\mathrm{V}}$ voneinander, wobei $d$ den Abstand der Akzeptoren darstellt. Diese Annahme wird als Minimumenergienäherung bezeichnet und ist nur gültig für $\mathrm{T}=0 \mathrm{~K}^{[13]}$. Durch langreichweitige Abschirmung ionisierter Donatoren und Akzeptoren (Dichten $N_{\mathrm{D}}, N_{\mathrm{A}}$ ) entsteht ein Raumladungszylinder mit dem Radius $R$, der durch die Neutralitätsbedingung gegeben ist.

$$
\begin{aligned}
R & =\sqrt{\frac{\kappa}{\pi\left|N_{\mathrm{D}}-N_{\mathrm{A}}\right|}}(, \text { Read-Radius“ mit }) . \\
\kappa & =\frac{f_{\mathrm{V}}}{d}
\end{aligned}
$$

READ ${ }^{[48]}$ berechnet die elektrostatische Verschiebung $-e \Phi_{\mathrm{d}}$ der Akzeptoren an der Versetzung zu

$$
E_{\mathrm{V}}(\kappa)=\frac{e^{2} \kappa}{2 \pi \varepsilon \varepsilon_{0}}\left(\frac{3}{2} \ln \left(\frac{\kappa}{\left(\pi\left(N_{\mathrm{D}}-N_{\mathrm{A}}\right)\right)^{1 / 3}}\right)-0.166\right) .
$$

Eine von ionisierten Donatoren oder Akzeptoren abgeschirmte kontinuierliche Linienladung besitzt im Abstand $r>d / f_{\mathrm{V}}$ zum Versetzungskern ein Potential (19], unter 
Vernachlässigung des Deformationspotentials)

$$
\Phi(r, \kappa)=-\frac{e \kappa}{2 \pi \epsilon \epsilon_{0}} \frac{1}{2}\left(\ln \left(\frac{R^{2}}{r^{2}}\right)-1+\frac{r^{2}}{R^{2}}\right) .
$$

Dieses Potential und dessen elektrisches Feld verschwinden an der Stelle $r=R$. Für $r>R$ ist $\Phi(r, \kappa)=0$. Für diese Lösung wird die Ladung entlang der Versetzung als kontinuierlich verteilt angesehen. $\mathrm{LABUSCH}^{[19]}$ vergleicht die Potentiale von Orten, an denen sich eine Ladung befindet, mit den Stellen zwischen zwei Ladungen und legt den inneren Abschneideradius für diese Beschreibung auf $\frac{1}{\kappa}$ fest. Diesen Zusammenhang verdeutlicht die Abb 2.5, in der $\Phi_{\mathrm{d}}^{(0)}$ das Potential am Ort der Versetzungen darstellt.

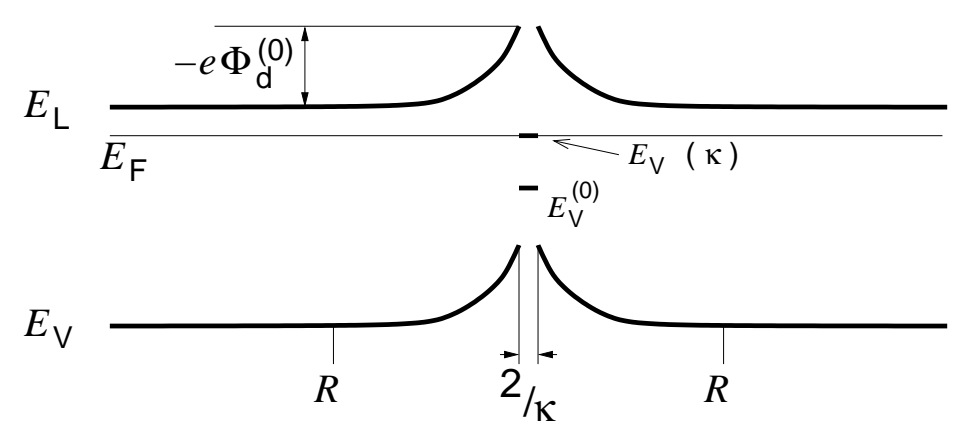

Abbildung 2.5 - Bandverbiegung einer geladenen Versetzung, $-e \Phi$ : Energiebarriere des elektrostatischen Potentials, $E_{\mathrm{V}}^{(0)}, E_{\mathrm{V}}\left(f_{\kappa}\right)$ : Energie der neutralen und geladenen Versetzung, $E_{\mathrm{V}}, E_{\mathrm{L}}$ : Bandkantenenergien, $E_{\mathrm{F}}:$ Fermi-Niveau, $R$ : Read-Radius, $\kappa$ bestimmt als Liniendichte der Ladungen im Versetzungskern gleichzeitig den inneren Abschneideradius.

Wie im Abschnitt 2.3.1.1 erwähnt, dissoziiert eine $60^{\circ}$-Versetzungen in eine $30^{\circ}$ - und eine $90^{\circ}$-Partialversetzung. Es ist davon auszugehen, dass die unterschiedlichen Versetzungskerndefekte der unterschiedlichen Partialversetzungen nicht wie im hier beschriebenen Modell nur einen Zustand sondern ein Spektrum von Zuständen in der Bandlücke erzeugen.

\subsubsection{Das elastische Verzerrungsfeld}

Das erste Modell über den Einfluss elastischer Verzerrungen auf die Bandstruktur ist von BARDeEn und Shockley ${ }^{[49]}$ vorgestellt worden. Dort wird gezeigt, dass die Beweglichkeit der Ladungsträger aufgrund von Streuprozessen von der Richtung und dem Betrag kleiner Verschiebungen der Atome aus ihrer mittleren Position sowie vom Quadrat der Wechselwirkungskonstante abhängt. Diese Wechselwirkungskonstanten, welche die modifizierte Bandstruktur beschreiben, werden als Deformationspotentiale $\Xi_{i j}$ bezeichnet. Mit den Komponenten des Verzerrungstensors $\epsilon_{i j}$ kann die Verschiebung der 
Leitungsbandkante mit $\Delta E_{\mathrm{L}, k}=\sum_{i, j} \Xi_{i j} \epsilon_{i j}$ berechnet werden. Wegen der Symmetrien des Diamantgitters und bei Betrachtung einer Kristallrichtung mit hoher Symmetrie $(\langle 100\rangle$, $\langle 111\rangle)$ reduziert sich die Anzahl der zu berücksichtigen Deformationspotentiale auf zwei:

$$
\Delta E_{\mathrm{L}}=\Xi_{\mathrm{d}} \cdot \operatorname{Sp}\{\epsilon\}+\Xi_{\mathrm{u}}(\widehat{k} \epsilon \widehat{k})
$$

Hier bezeichnen $\operatorname{Sp}\{\epsilon\}$ die Spur des Spannungstensors $\epsilon$ und $\widehat{k}$ einen reziproken Einheitsvektor entlang einer $\langle 100\rangle$-Richtung. Für den Fall eines elastisch isotropen Materials ist $\widehat{k} \epsilon \widehat{k}=0$ und $\Delta E_{\mathrm{L}}$ ist wie die hydrostatische Komponente des Verzerrungsfeldes (siehe Abschnitt 2.3.1.2) eine Funktion von $\frac{\sin \Theta}{r}[13]$ :

$$
\Delta E_{\mathrm{L}}=-\frac{b \Xi_{\mathrm{d}}(1-2 \nu)}{2 \pi(1-\nu)} \frac{\sin \Theta}{r}
$$

Da die in ${ }^{[49]}$ berechnete Beweglichkeit vom Quadrat des Deformationspotentials abhängt, geht dessen Vorzeichen nicht aus dieser Berechnung hervor. Das Vorzeichen von $\Xi_{\mathrm{d}}$ wird in der Literatur kontrovers diskutiert, so dass nicht klar ist, in welche Richtungen sich die Bandkanten im Dilatations- bzw. im Kompressionsfeld der Versetzung verschieben $\stackrel{[13]}{\text {. }}$ Abb. 2.6 zeigt die Bandstruktur in der Nähe einer Stufenversetzung. Wie aus der Ab-

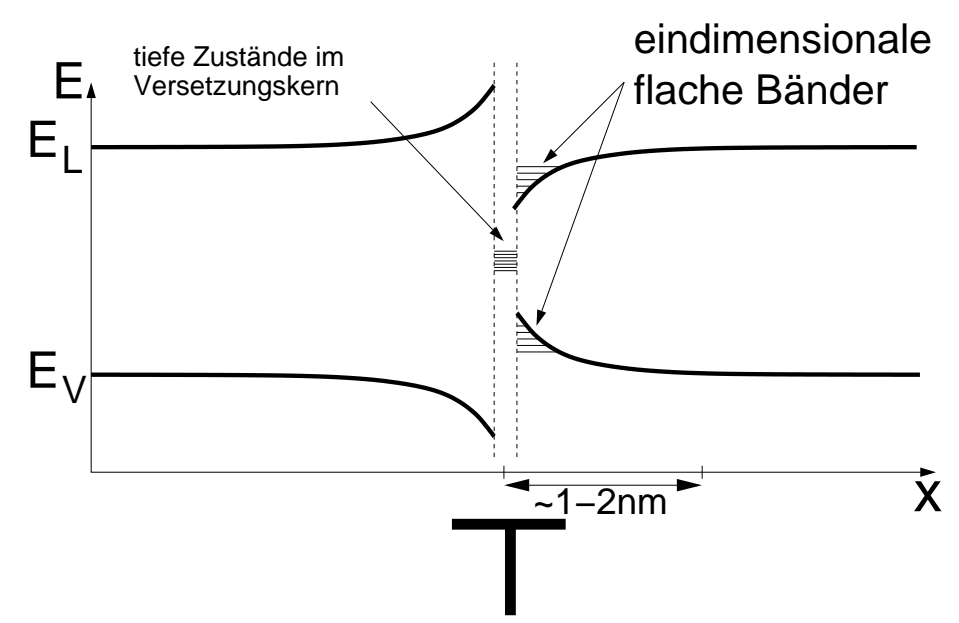

Abbildung 2.6 - Bandstruktur in der Nähe einer Versetzung, $E_{\mathrm{V}}, E_{\mathrm{L}}$ bezeichnen das Valenz- bzw. das Leitungsband. Die Ausdehnung der Bandverbiegung beträgt in Silizium etwa 1-2nm ${ }^{[13]}$. Infolge der Bandverbiegung kommt es auf einer Seite zur Ausbildung eindimensionaler flacher Bänder.

bildung hervor geht, führt die aus dem Deformationspotential resultierende Verbiegung der Bänder auf einer Seite der Versetzungen zu der Ausbildung eindimensionaler Bänder. Die Energiedifferenz zwischen den Bandkanten $E_{\mathrm{V}}$ und $E_{\mathrm{L}}$ und den Kanten dieser 
Bänder liegt jeweils im Bereich zwischen $\approx 70 \mathrm{meV}$ und $80 \mathrm{meV}^{[12]}$. Aufgrund ihrer Nähe zu den Bandkanten werden diese Bänder als „flache Bänder“ bezeichnet.

\subsection{Wechselwirkungen metallischer Verunreinigungen mit Versetzungen}

Versetzungen mit rekonstruierten Bindungen im Versetzungskern und ohne Dekoration mit Fremdatomen besitzen nur bei Temperaturen unter 100K eine vergleichsweise geringe Rekombinationsaktivität. Diese wird in Zusammenhang mit den eindimensionalen flachen Bändern gebracht (siehe Kap. 2.3.2.2). Existieren tiefe Zustände an den Versetzungen, so gibt es eine deutliche Zunahme der Rekombinationsaktivität von Versetzun-

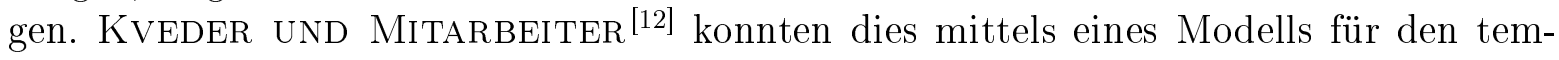
peraturabhängigen Kontrast von elektronenstrahlinduziertem Kurzschlussstrom (EBIC, „Electron Beam Induced Current") von unterschiedlich stark dekorierten Versetzungen zeigen. Dies kann als Hinweis gewertet werden, dass es sich bei den tiefen Zuständen, auf deren Basis das Modell den EBIC-Kontrast beschreibt, um metallische Fremdatome handelt

Im folgenden sollen unterschiedlichen Wechselwirkungen metallischer Verunreinigungen mit Versetzungen zusammengefasst werden, die zum einen Möglichkeiten der Anreicherung und zum anderen die elektrischen Eigenschaften der Metallatome an Versetzungen erklären. Detailliertere Beschreibungen dazu finden sich bei SEIBT UND MiTARBEITERN ${ }^{[16,50]}$. Auf den Einfluss von Versetzungen auf die Diffusion und die Löslichkeit von Gold im speziellen wurde bereits im Kap. 2.2.1 im Rahmen der Beschreibung der Eigenschaften von Gold in Silizium eingegangen.

\subsubsection{Chemische und strukturelle Wechselwirkungen}

Metallische Fremdatome können an Versetzungen sowohl im Versetzungskern als auch im Verzerrungsfeld aufgrund dessen struktureller Eigenschaften gebunden werden. Im Versetzungskern gebundene Metallatome können unterschiedliche Konfigurationen annehmen, da der Versetzungskern gemäß Kap. 2.3.1.1 aufgrund der Aufspaltung in Partialversetzungen verschiedene Konfigurationen für chemische Bindungen zu Verfügung stellt. Somit hängt die Symmetrie des im Versetzungskern gebundenen Metallatoms von der dort eingenommenen Konfiguration ab. Daher ist zu erwarten, dass sich die elektronische Struktur eines Fremdatoms im Versetzungskern grundlegend von der eines Fremdatoms im ungestörten Kristall unterscheidet. FuJITA UND MitARBEITER haben mittels theoretischer Studien die Bindungsenergie von einzelnen Kupferatomen ${ }^{[14]}$ und Goldatomen $\stackrel{[15]}{1 m}$ Versetzungskern berechnet. Für Kupfer sind demnach Bindungsenergien von etwa $2 \mathrm{eV}$ und für Gold zwischen $1.71 \mathrm{eV}$ und $2.13 \mathrm{eV}$ je nach berechneter Konfiguration zu erwarten. SeibT Und MitarbeiteR $\stackrel{[16]}{ }$ berechnen die Linienkonzentration von Gold im 
Versetzungskern, die sich bei $800^{\circ} \mathrm{C}$ in Abhängigkeit von der Bindungsenergie für eine bestimmte Gesamtkonzentration einstellt. Dabei zeigte sich, dass bei einer Bindungsenergie von etwa $1.5 \mathrm{eV}$ die Hälfte allen Goldes und bei einer Bindungsenergie von $2 \mathrm{eV}$ nahezu das gesamte Gold im Versetzungskern gebunden ist.

Eine weitere bereits im Kap. 2.2.1 für Gold beschriebene Eigenschaft der Versetzungen stellt die sogenannten Möglichkeit der „Pipe diffusion“ dar. Dabei stellt der Versetzungskern einen Kanal dar, durch den sich Fremdatome mit erhöhter Diffusivität bewegen können ${ }^{[40]}$, was die Anreicherung von Fremdatomen im Siliziummaterial beschleunigen kann.

Die Bindung im Verzerrungsfeld erfolgt im wesentlichen infolge des Größenunterschiedes zwischen den kovalenten Radien der Fremdatome und denen der Siliziumatome, was zu einer elastischen Wechselwirkung führt (,Size effect“). Dabei hängt die Wechselwir-

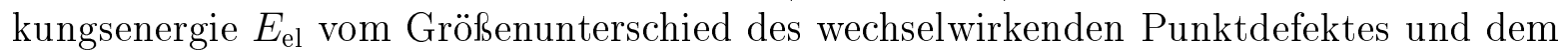
Atom des Wirtsgitters mit den Radien $r_{\mathrm{pd}}, r_{0}$ ab $\stackrel{[17]}{ }$ :

$$
E_{\mathrm{el}}(r, \Theta)=4 \mu b \epsilon r_{0}^{3} \frac{\sin \Theta}{r}
$$

Hier stellen $\mu$ den Schubmodul und $b$ den Betrag des Burgersvektors dar. $\epsilon=\frac{r_{0}-r_{\mathrm{pd}}}{r_{0}}$ bezeichnet die normierte Differenz der kovalenten Radien. Mit der Punktdefektkonzentration im ungestörten Gitter $n_{\mathrm{pd}}^{0}$ ist

$$
n_{\mathrm{pd}}(r, \Theta)=n_{\mathrm{pd}}^{0} \cdot \exp \left(\frac{E_{\mathrm{el}}(r, \Theta)}{k_{\mathrm{B}} T}\right)
$$

die Gleichgewichtsverteilung der Punktdefekte im Verzerrungsfeld, die sich bei einer Diffusionstemperatur $T$ einstellt.

Für substitutionelles Gold fanden Bullough UND NEWMAN ${ }^{[17]}$ eine maximale Wechselwirkungsenergie von $0.85 \mathrm{eV}$. Der Radius, bei dem die Wechselwirkungsenergie auf $k_{\mathrm{B}} T$ $\left(k_{\mathrm{B}}\right.$ : Boltzmannkonstante, $T$ : Temperatur) abgefallen ist, wird dort für Gold im Verzerrungsfeld mit $2.6 \mathrm{~nm}$ bei $1200^{\circ} \mathrm{C}$ angegeben.

\subsubsection{Elektrische Wechselwirkungen}

Bei der Beschreibung der elektrischen Wechselwirkungen muss wiederum zwischen solchen Atomen, die chemisch im Versetzungskern, und solchen, die im Verzerrungsfeld gebunden sind, unterschieden werden. Der Fall der Metallatome im Versetzungskern wurde bereits bei der Beschreibung der elektrischen Eigenschaften von Versetzungen im Kap. 2.3.2.1 abgehandelt. Dort wird beschrieben, wie sich geladene Defekte, die sowohl intrinsisch als auch extrinsisch sein können, auf die Bandstruktur in der Umgebung der Versetzung auswirkt. Für Gold im speziellen haben FuJita Und MitARBEITER ${ }^{[15]}$ die 
Energiezustände berechnet, die als gebundener Zustand im Versetzungskern zu erwarten sind. Das Ergebnis war je nach betrachteter Konfiguration ein Donatorzustand mit einer Energie zwischen $0.61 \mathrm{eV}$ und $0.7 \mathrm{eV}$ über der Valenzbandkante und ein Akzeptorzustand mit $0.53 \mathrm{eV}$ bis $0.74 \mathrm{eV}$ unterhalb der Leitungsbandkante (Abb. 2.7). Nach diesen

$E_{L}$

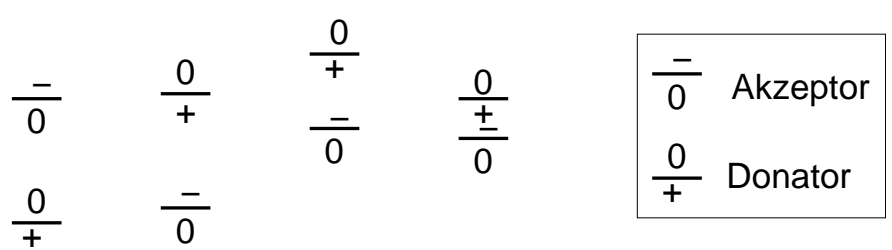

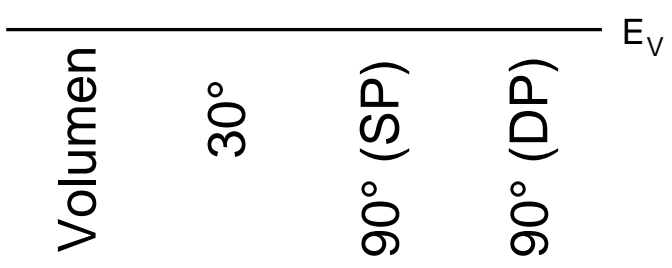

Abbildung 2.7 - Berechnete Energienivaus einzelner Goldatome, die in unterschiedlichen Konfigurationen im Versetzungskern gebunden sind ${ }^{[15]}$ (Die Bezeichnungen SP (,single period“) und DP (,,double period") bezeichnen unterschiedliche Möglichkeiten der Rekonstruktion ungesättigter Bindungen im Versetzungskern).

Berechnungen stellen die Zustände des im Kern gebundenen Goldes ein sogenanntes negatives-U-Zentrum dar, bei der sich durch die Beteiligung von Gitterrelaxationen die Reihenfolge der Elektronenzustände umkehren kann $\underline{\text { [51] }}$.

Wie im Kap. 2.3.2.2 beschrieben, resultiert aus der Verzerrung des Gitters im Verzerrungsfeld einer Versetzung ein Deformationspotential $\Xi_{\mathrm{d}}$, das die Leitungs- und Valenzbandzustände relativ zueinander verschiebt (Gl. (2.33)). Die Verzerrung führt aufgrund eines Deformationpotentials $\Xi_{\mathrm{pd}}$ ebenfalls zu einer Verschiebung der Defektzustände. Daraus resultiert eine ortsabhängige relative Verschiebung $\Delta E_{\mathrm{L} \text {,pd }}$ der Bandzustände und der Defektzustände ${ }^{[13]}$ :

$$
\Delta E_{\mathrm{L}, \mathrm{pd}}=-\frac{b\left(\Xi_{\mathrm{d}}-\Xi_{\mathrm{pd}}\right)(1-2 \nu)}{2 \pi(1-\nu)} \frac{\sin \Theta}{r}
$$

Die Wirkung des Verzerrungsfeldes auf die Bandzustände und auf die Defektzustände der Metallatome sind im allgemeinen unterschiedlich $\left(\Xi_{\mathrm{d}} \neq \Xi_{\mathrm{pd}}\right)$. Für den Goldakzeptorzustand haben SAMARA UND BARNES ${ }^{[52]}$ ermittelt, dass sich die Energiedifferenz dieses 
Niveaus unter hydrostatischem Druck sowohl zum Leitungsband als auch zum Valenzband verändert, betonen aber, dass in anderen Arbeiten abweichende Ergebnisse erzielt wurden.

SCHRÖTER UND CERvA ${ }^{[13]}$ vergleichen die Zustandsdichten von Punktdefektwolken, die sich in der Annahme bezüglich einer elastischen Wechselwirkung unterscheiden: In der einen Betrachtung wird eine elastische Wechselwirkung vernachlässigt (Gl. (2.35): $E_{\text {el }}(r, \Theta)$ konstant innerhalb des Verzerrungsfeldes), in der anderen wird eine elastische Wechselwirkung zugelassen, wobei die Atome dann ihre Gleichgewichtsverteilung im Verzerrungsfeld gemäß Gl. (2.35) eingenommen haben. Sie kamen zu dem Ergebnis, dass die Zustandsdichte stark von der elastischen Wechselwirkung beeinflusst wird. Aus diesen unterschiedlichen Zustandsdichten berechneten sie das zu erwartende DLTS-Spektrum dieser Punktdefektwolke. Es zeigte sich, dass auf diese Weise eine verbreiterte und asymmetrische DLTS-Linie, die in n-dotierten versetzungshaltigen Proben gemessen wird, simuliert werden konnte. Diese DLTS-Linie wurde zum ersten mal von KIMERLING UND PATEL ${ }^{[53]}$ beschrieben. Dort wurde DLTS an versetzungshaltigem Silizium gemessen und die Spektren nach unterschiedlichen Auslagerungsexperimenten verglichen. Diese Linie blieb nach einer Auslagerung von einer Stunde bei $900^{\circ} \mathrm{C}$ als asymmetrisch verbreiterte DLTS-Linie von den vormals fünf Linien übrig. Bei systematischen Untersuchungen versetzungsinduzierter DLTS-Linien in Silizium von OMLING UND MitARBEITERN [54] wird für diese Linie die Bezeichnung C-Linie eingeführt, die in manchen Fällen als Doppellinie $\mathrm{C}_{1}$ und $\mathrm{C}_{2}$ auftritt $\underline{[54} \underline{-56]}$. KVEDER Und Mitarbeiter ${ }^{[57]}$ konnten zeigen, dass die Konzentration der zugehörigen Defekte durch Aluminiumgettern deutlich reduziert werden konnte. Außerdem besitzen diese Defekte die Eigenschaften ausgedehnter Defekte mit lokalisierten Zuständen (siehe Kap. 2.1.1). SEIBT und Mitarbeiter ${ }^{[58]}$ zeigen einen Zusammenhang zwischen der C-Linienamplitude und der Geschwindigkeit, mit der die Versetzungen vor der DLTS-Messung durch unterschiedliche Belastungen während einer 4-Punkt-Biegung bewegt worden sind. Bei dem Vergleich zweier unterschiedlicher Geschwindigkeiten $(10 \mu \mathrm{m} / \mathrm{min}, 2 \mu \mathrm{m} / \mathrm{min})$ konnte festgestellt werden, dass die geringere Geschwindigkeit zu einem Anstieg und die höhere Geschwindigkeit zu einer Abnahme dieser Amplitude führte. Erklärt wurde dieser Befund damit, dass unterhalb einer kritischen Geschwindigkeit Verunreinigungen von den Versetzungen „eingesammelt" werden, was zu einem Anstieg der Amplitude führt. Geschwindigkeiten oberhalb eines kritischen Wertes führen dann zu einer Verringerung der Dekoration mit Verunreinigungen, da diese dann nicht mehr imstande sind, den Versetzungen zu folgen. Es wird somit davon ausgegangen, dass es sich bei diesen Defekten um metallische Verunreinigungen an Versetzungen handelt. Neben dieser C-Linie werden noch weitere DLTS-Linien in versetzungshaltigem n-dotierten Silizium gemessen: die A-, B- und die D-Linie. Die Amplitude der D-Linie zeigt eine logarithmische Abhängigkeit von der Einfangzeit ${ }^{[54]}$ und die Zustandsdichte der zugehörigen Defekte eine Linienbreite von $22.5 \mathrm{meV}{ }^{[59]}$. Voss ${ }^{[60]}$ vergleicht die Emissionskinetik der D-Linie unterschiedlicher Autoren mit einer dort gefundenen Linie, die ebenso in versetzunghaltigem, n-dotierten Silizium gemessen wurde 
und einem goldinduziertem Defekt zugeordnet wird. Die Emissionskinetiken stimmten im Rahmen der Messgenauigkeit überein. Eine Aussage über die Identität dieser Defekte wird aber nicht getroffen. Eine Zusammenfassung der Eigenschaften dieser und anderer versetzungsbedingter Linien findet sich bei SCHRÖTER UND CERvA ${ }^{[13]}$. 


\section{Probenpräparation}

Da Wechselwirkungen von Gold und Versetzungen untersucht werden sollen, wurden zunächst Versetzungen durch statische Kompression eines Siliziumquaders erzeugt (Kap. 3.2). Aus diesem Quader wurden anschließend jeweils zwei Proben in einer bestimmten Weise entnommen (Kap. 3.3) und anschließen mit Gold bedeckt. Durch Diffusion wurden die Proben definiert mit Goldatomen kontaminiert (Kap. 3.4). Um die Untersuchung einer Abhängigkeit der untersuchten Effekte von der Goldkonzentration zu ermöglichen, wurden die goldbedeckten Oberflächen der Proben anschließend unter einem bestimmten Winkel schräg angeschliffen, um eine Oberfläche zu erzeugen, auf der die Goldkonzentration lateral variiert (Kap. 3.5). Die DLTS-Methode erfordert Kontakte mit einer Potentialbarriere. Daher wurden dann auf diesen Oberflächen Schottky-Kontakte präpariert (Kap. 3.6).

Zur Bestimmung der Versetzungsdichten musste an bestimmten Flächen eine Defektätze vorgenommen werden, was in Kap. 3.7 beschrieben wird. Da auch einige Transmissionselektronenmikroskopieuntersuchungen (TEM) durchgeführt wurden, wird im Kap. 3.8 kurz auf die Anforderungen an die TEM-Probe eingegangen.

\subsection{Allgemeines}

Ausgangsmaterial für alle Proben war zonengezogenes, phosphor- (n-Typ) bzw. bordotiertes (p-Typ) Silizium. Mussten Proben gesägt werden, so wurden Sägeschäden durch Läppen mit Emulsionen aus Wasser und Borcarbidpulver mit 600er und 1000er Körnungen entfernt. Probenschliffe erfolgten auf siliziumcarbidbeschichteten Schleifscheiben mit 500er und 1000er Körnungen.

Chemische Reinigungen verliefen nach folgendem Rezept ${ }^{[61]}$ : Zunächst wurden die Proben drei mal vier Minuten in $\mathrm{H}_{2} \mathrm{O}$ (dest.) und je fünf Minuten in Aceton und Methanol (beide in „pro analysi“-Qualität) gereinigt, um zunächst grobe Verunreinigungen und Fettrückstände zu entfernen. Um Rückstände der organischen Lösungsmittel zu beseitigen, wurden die Proben dann eine Minute $\mathrm{H}_{2} \mathrm{O}$ (dest.) gereinigt. Alle diese Reinigungsschritte erfolgten im Ultraschallbad. Abgeschlossen wurde die Reinigung durch einen 45 Sekunden dauernden Ätzschritt in $\mathrm{HF}: \mathrm{HNO}_{3}$ (1:7, HF: 40\%, $\mathrm{HNO}_{3}: 65 \%$ ). Als HF-Dip wird ein 30 Sekunden langer Ätzschritt in HF: $\mathrm{H}_{2} \mathrm{O}$ (1:10, HF: 40\%) bezeichnet. Das zum Ätzen von Gold benutzte Königswasser hatte die Zusammensetzung $\mathrm{HCl}: \mathrm{HNO}_{3}$ (1:3, $\mathrm{HCl}: 30 \%, \mathrm{HNO}_{3}$ : 65\%). Sämtliche Probenglühungen wurden unter einem konstanten 
Argonfluss durchgeführt. Die Reinheit des Argongases betrug 99.999\%.

\subsection{Erzeugung von Versetzungen}

Aus Siliziumscheiben mit bekannter Kristallorientierung wurden Stäbchen der Größe von

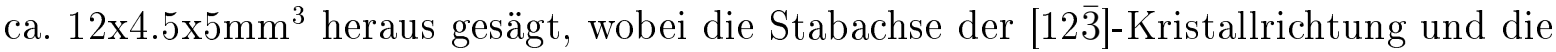

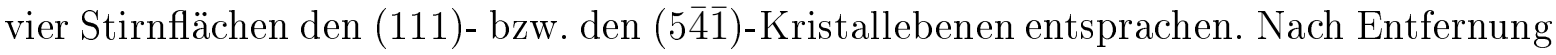
der Sägeschäden wurden die Stirnflächen mit Diamantpasten der Körnungen $7 \mu \mathrm{m}$ und

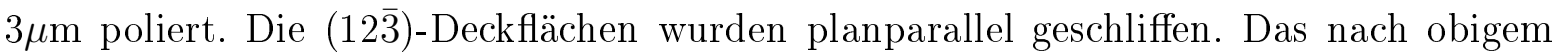
Rezept chemisch gereinigte und geätzte Probenstäbchen wurde zwischen zwei Quarzglasplättchen (suprasil) mit planparallelen Oberflächen, die ebenfalls chemisch gereinigt und anschließend für eine Minute in $\mathrm{HF}: \mathrm{H}_{2} \mathrm{O}$ (1:10) geätzt wurden, in die Verformungsapparatur eingebaut.

Das Einbringen der Versetzungen erfolgte bei $800^{\circ} \mathrm{C}$ durch statische Kompression in

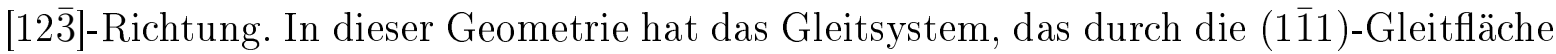
und die [101]-Gleitrichtung gegeben ist, mit 0.466 den höchsten Schmidfaktor aller Gleitsysteme dieses Typs. Die Gleitrichtung entspricht der Richtung des Burgersvektors der $60^{\circ}$-Versetzungen entlang der [110]- oder der [011]-Richtung. Die Verformungsspannung, mit der die Probenstäbchen verformt wurden, betrug entweder 23MPa oder 34MPa. Die Verformungen wurden bis zum Beginn des Verfestigungsbereiches durchgeführt. Die rechnerische Versetzungsdichte betrug demnach ca. $1.6 \cdot 10^{8} \mathrm{~cm}^{-2}$ bzw. $3.5 \cdot 10^{8} \mathrm{~cm}^{-2}[62]$. Durch Anheben der Last wurden die Deformationsprozesse beendet. Die Abkühlung der Probe erfolgte im geöffneten Verformungsofen mit einer durchschnittlichen Abkühlrate von ca. $0.8 \mathrm{~K} / \mathrm{s}$ bis $200^{\circ} \mathrm{C}$.

\subsection{Probenentnahme aus den deformierten Stäbchen}

Nach der Deformation waren unter einem optischen Mikroskop im Dunkelfeldmodus Gleitstufen auf den betrachteten Stirnflächen zu erkennen. Entlang der Hauptgleitebenen wurden mit einer Well-Diamantdrahtsäge drei Schnitte in der Stabmitte durchgeführt, so dass zwei Proben mit einer Dicke von ca. $1 \mathrm{~mm}$ entnommen werden konnten (Abb. 3.1). Dazu wurde das Stäbchen auf eine Vorrichtung geklebt, die die entsprechenden Winkel zwischen dem Stäbchen und der Schnittfläche einstellt.

Als Probenvorderseiten werden im folgenden die Seiten der so entstandenen Proben bezeichnet, die der „Partnerprobe“, also der Probe, die dem gleichen Stäbchen entstammt, vor dem Sägen zugewandt war. Als Probenrückseiten gelten entsprechen die voneinander abgewandten Seiten. Nach dem Sägen wurden die Sägeschäden auf der Vorderseite mit 1000er Borcarbid und die der Rückseite mit 600er Borcarbid entfernt. Für die Rückseiten ist eine größere Rauhigkeit für den später aufzureibenden ohmschen Rückseitenkontakt 
von Vorteil. Die Abweichung des Winkels der erzielten Probenoberfläche zur gewünschten Hauptgleitebene wurde durch spätere TEM-Aufnahmen (Transmissionselektronenmikroskopie) auf unter $5^{\circ}$ abgeschätzt.

Die so hergestellten Proben besitzen an ihren Vorderseiten vergleichbare Eigenschaften bezüglich der Versetzungsdichte und der Versetzungsverteilung. Außerdem verlaufen die Versetzungen im wesentlichen parallel zur Probenoberfläche und damit weitgehend parallel zu den später zu präparierenden Goldschichten bzw. Schottky-Kontakten. Auf diese Weise wird zum einen die modifizierte Diffusion entlang der Versetzungen in den Hauptgleitebenen während der Goldeindiffusion aus der Goldschicht („Pipe diffusion““ㅆ0]) unterdrückt. Zum anderen kann unerwünschter Ladungstransport entlang dieser Versetzungen unter dem Einfluss des elektrischen Feldes der Raumladungszone eines SchottkyKontaktes minimiert werden.

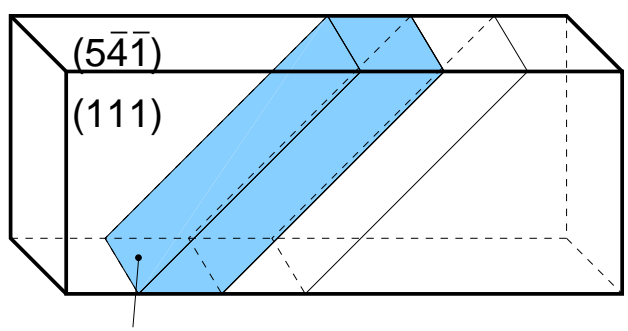

(1111)-Gleitebene

Abbildung 3.1 - Probenstäbchen nach der Deformation vor der Probenentnahme: Aus der Mitte des Stäbchens werden zwei Proben parallel zu den Hauptgleitebenen ausgesägt

\subsection{Goldeindiffusion}

Die geläppte Probenvorderseite wurde mit Diamantpasten der Körnungen $7 \mu \mathrm{m}, 3 \mu \mathrm{m}$, $1 \mu \mathrm{m}$ poliert. Darauf folgte eine chemische Reinigung und Ätze nach dem oben beschriebenen Rezept mit anschließendem HF-Dip. Fixiert in einer zuvor ebenfalls chemisch gereinigten und in Königswasser geätzten Teflonaufnahme wurde die Probe in eine Aufdampfkammer eingebaut. Der Druck in der Kammer hatte nach weniger als fünf Minuten nach dem HF-Dip einen Wert unter $10^{-4}$ mbar. Das Gold mit einer Reinheit von $99.99 \%$ wurde vor dem Einbau ca. eine Minute in Königswasser geätzt. Die Schichtdicke nach dem Aufdampfen war größer als 100nm. Nach dem Aufdampfen wurde die Probe sofort in das Glühgestänge aus Quarzglas in Suprasil-Qualität des entsprechenden Diffusionsofens eingebaut.

Die Eindiffusion erfolgte im Falle versetzungshaltiger Proben bei $800^{\circ} \mathrm{C}$ für 17 Stunden. Beendet wurde die Diffusionsglühung je nach gewünschter Abkühlrate durch unterschiedliche Abkühlprozesse. Die abgeschreckten Proben wurden durch einen Sturz in 
Siliconöl aus einem Vertikalofen mit ca. $500 \mathrm{~K} / \mathrm{s}$ abgekühlt.

Die versetzungsfreien Proben wurde das Gold bei $850^{\circ} \mathrm{C}$ für 10 Stunden eindiffundiert. Die Abkühlung erfolgte im Ofengestänge, das aus dem Ofen herausgezogen worden war. Die Abkühlrate betrug in diesem Fall ca. $1.2 \mathrm{~K} / \mathrm{s}$.

\subsection{Schrägschliff}

Bei der Goldeindiffusion wurde ein Konzentrationprofil eingestellt. Um diese Konzentrationabhängigkeit zum Abstand der Goldschicht auszunutzen, wurde die Vorderseite der Probe nach der Diffusionsglühung schräg abgeschliffen (Abb. 3.2). Die Winkel zwischen der alten und der neuen Probenoberfläche betrug maximal ca. $4^{\circ}$. An der Spitze wurde wegen der Positionsbestimmung der Kontakte als Höhenreferenz eine kleine Ecke mit der Goldschicht stehen gelassen.

a

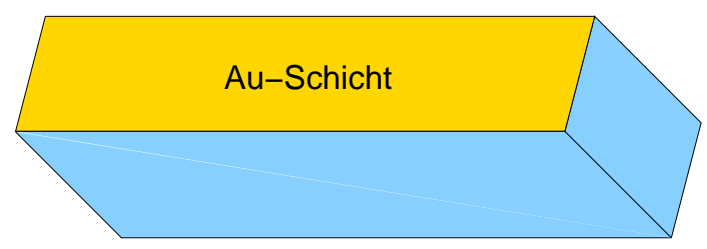

Schottky-Kontakte

b

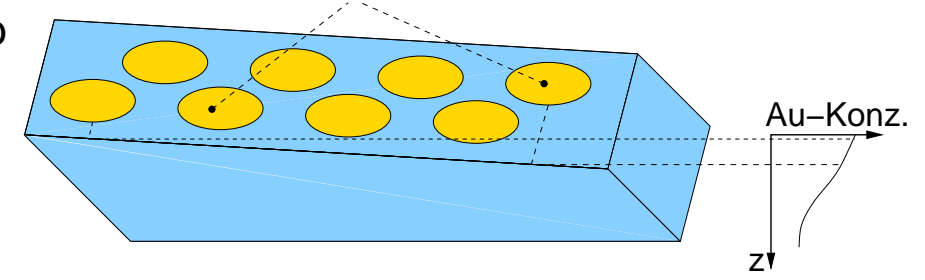

Abbildung 3.2 - a) Probe mit Goldschicht, b) Probe nach Schrägschliff und Präparation der Schottky-Kontakte: Die Kontakte befinden sich in Bereichen unterschiedlicher Goldkonzentration.

\subsection{Kontaktpräparation}

Die mit 1000er Siliziumcarbid geschliffene Fläche wurde in mehreren Schritten mit Diamantpasten der Körnungen $7 \mu \mathrm{m}, 3 \mu \mathrm{m}, 1 \mu \mathrm{m}$ und $0.25 \mu \mathrm{m}$ poliert, chemisch gereinigt und die Siliziumoxidschicht mit einem HF-Dip geätzt. Die n-Typ-Proben wurden dann sofort in die Aufdampfanlage eingebaut und durch eine Kontaktmaske mit Gold bedampft. Die p-Typ-Proben wurden nach dem HF-Dip für 25 Minuten entweder im Abstand von etwa 
$8 \mathrm{~cm}$ unter einer $50 \mathrm{~W}$-Halogenlampe $\left(\mathrm{ca} .45^{\circ} \mathrm{C}\right)$ oder bei Raumtemperatur ohne Lichteinfall gelagert, um die Ausbildung einer reproduzierbaren Oxidschicht zu ermöglichen. Anschließend wurden die Proben durch eine Kontaktmaske mit Aluminium bedampft. Der Druck im Rezipienten der Aufdampfanlage war zu Beginn der Metallverdampfung immer unter $10^{-4}$ mbar. Vor dem Schrägschliff wurde die Höhe der Proben mit Hilfe der Fokussierung eines optischen Mikroskops und der Höheneinstellung des Mikroskoptisches, auf der die Höhe in Mikrometereinheiten abgelesen werden kann, bestimmt. Dieser Vorgang wurde jeweils nach dem Schrägschliff wiederholt. Anhand des Referenzpunktes der Originalhöhe (siehe Kap. 3.5) konnte so an jedem Ort auf der schräg geschliffenen Probe der Abstand zur Diffusionsschicht bestimmt werden. Die Probe wurde nach dem Aufdampfen der Kontakte mit einer auf dem optischen Mikroskop angebrachte CCD-Kamera fotografiert. Mit Hilfe des Fotos konnten die Kontaktpositionen auf der Oberfläche und somit der Abstand zur Diffusionsschicht bestimmt werden.

Durch Aufreiben einer Aluminium-Gallium-Mischung auf die Rückseite der DLTSProbe wurde der ohmsche Rückseitenkontakt erzeugt. Die Probe ist dann mit Leitsilber auf der Kupferfläche eines Probenhalters fixiert worden. Der Metall-Halbleiter- bzw., im Falle von p-Typ Proben, Metall-Oxid-Halbleiterkontakt wurde durch Aufkleben eines Golddrahtes mit dem Durchmesser 0.1mm mit Leitsilber kontaktiert.

\subsection{Defektätzen an verformten Proben}

Alle Proben, die einer Defektätze unterzogen wurden, sind auf den zu untersuchenden Flächen zunächst mit 1000er Borcarbid geschliffen und in der gleichen Weise wie bei der Kontaktpräparation poliert worden. Nach der chemischen Reinigung erfolgte das Defektätzen mit einer Secco-Ätzlösung ${ }^{[63]}$ zum Erzeugen von Ätzgrübchen wie bei RudoLF ${ }^{[4]}$ beschrieben jedoch mit dem Unterschied, dass der Ätzvorgang nach 15 Sekunden beendet wurde.

Zur Bestimmung der Versetzungsdichten wurde von den verformten Proben, an denen zuvor DLTS gemessen wurde, Proben entnommen. Dazu wurde entlang der Richtung des Burgersvektors der $60^{\circ}$-Versetzungen ein Streifen abgesägt (siehe Abb. 3.3). Die so entstandene Schnittfläche entsprach der [121]-Fläche. Von der so entnommenen Probe wurden die Schnittfläche und daran planparallel die gegenüberliegende Fläche zum Defektätzen präpariert.

In gleicher Weise wurden von einer Probe, die, wie in Kap. 3.2 beschrieben, mit 34MPa Belastung verformt worden ist, vier Proben entnommen. Diese Proben wurden in gleicher Weise mechanisch bearbeitet und chemisch gereinigt, wie oben beschrieben. Drei dieser Proben wurden anschließend unter Schutzgas bei $900^{\circ} \mathrm{C}$ für eine Stunde, bei $1000^{\circ} \mathrm{C}$ für 37 Minuten oder bei $1100^{\circ} \mathrm{C}$ für 27 Minuten in Anlehnung an die von RodRIGUEz Und Mitarbeiter $[20]$ für ihre Proben verwendeten Parameter geglüht. Nach nochmaliger mechanischer und chemischer Behandlung standen so vier Proben mit Versetzungen zur 


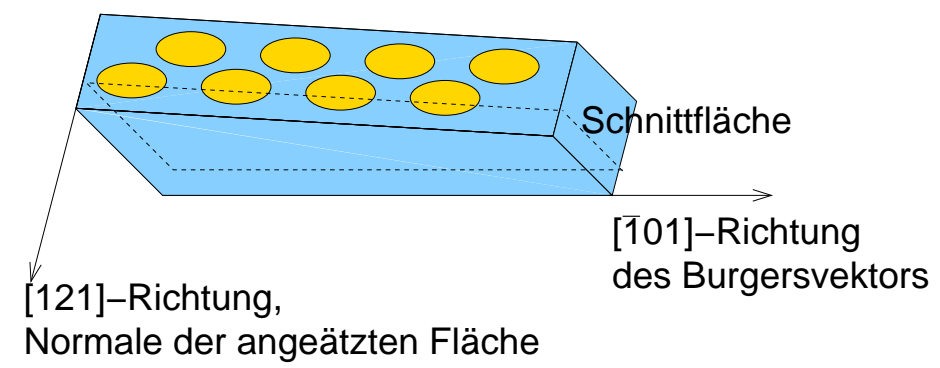

Abbildung 3.3 - Geometrie und Präparation der Probe, von der die Versetzungsdichte bestimmt wurde

Verfügung, an denen die Versetzungsdichte nach unterschiedlichen Auslagerungsexperimenten gemessen werden sollte. Die an die Arbeit von Rodriguez Und Mitarbeiter angelehnten Parameter sollten die Vergleichbarkeit unter den Experimenten vereinfachen. Die zunächst nicht ausgelagerte Probe wurde anschließend nach mechanischer und chemischer Behandlung bei $800^{\circ} \mathrm{C}$ für 17 Stunden geglüht, um auch hier die Entwicklung der Versetzungsdichte während einer Glühung zu messen, die der Glühung für die Goldeindiffusion entsprach.

\subsection{Präparation der TEM-Probe}

Die TEM-Probe (Transmissionselektronenmikroskopie) sollte in der Weise präpariert werden, dass die Richtung des Elektronenstrahls mit der Richtung einer der zwei möglichen $60^{\circ}$-Versetzungslinien übereinstimmt. Demzufolge musste die präparierende Sägefläche eine (011)- oder (110)-Fläche darstellen. Außerdem sollte eine möglichst hohe Goldkonzentration vorliegen. Dazu wurde auf die Probe mit der höheren zu erwartenden Versetzungsdichte nach erfolgten DLTS-Messungen zunächst ein Schutzstück aus einer Siliziumscheibe aufgeklebt und dann möglichst nahe an der Diffusionsschicht eine TEMProbe gemäß Abb. 3.4 heraus gesägt. Die weitere Präparation erfolgte wie bei dem von SEIBT ${ }^{[64]}$ beschriebenen Verfahren. 


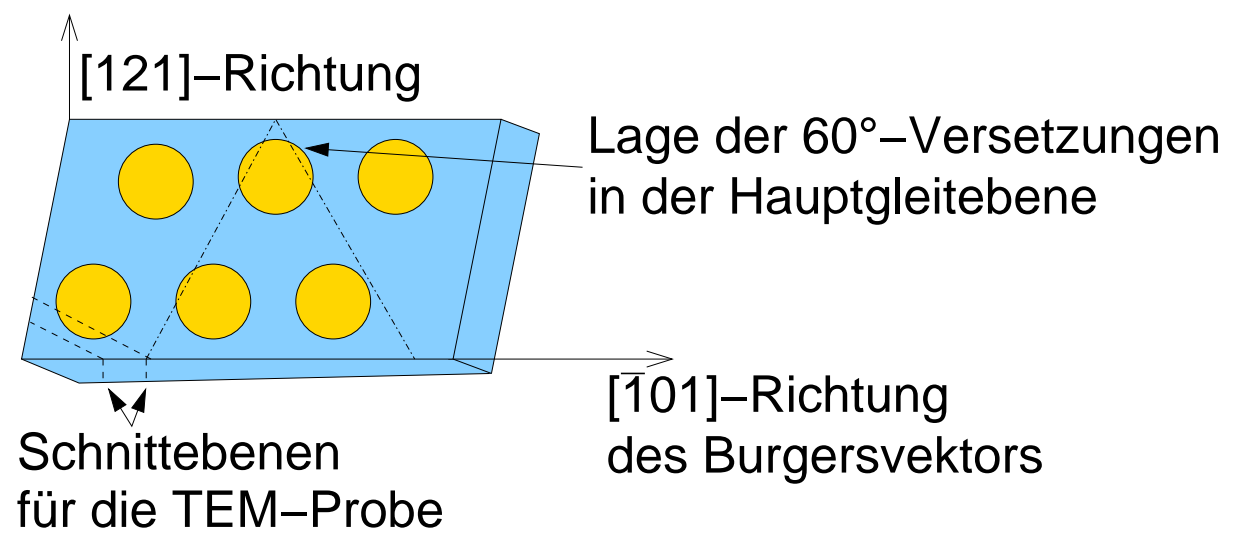

Abbildung 3.4 - Präparation der TEM-Probe aus einer DLTS-Probe nach erfolgter DLTS-Messung. Die TEM-Probe wurde so heraus gesägt, dass die zu beobachtende Stelle möglich nahe an der ehemaligen Diffusionsschicht lag. Die Richtung des Elektronenstrahls sollte der Richtung der Versetzungslinien entsprechen. 


\section{Ergebnisse}

In diesem Kapitel werden zunächst die Resultate der Bestimmung von Versetzungsdichten unterschiedlicher Proben gezeigt. Die darauf folgenden Ergebnisse der DLTSMessungen sind an Proben mit zum Teil unterschiedlichen Versetzungsdichten erzielt worden, um deren Einfluss auf die mögliche Segregation von Goldatomen an den Versetzungen zu untersuchen. Darüber hinaus wurde die Versetzungsdichte einer Probe bestimmt, die aus der Arbeit von Rodriguez UND MitARBEITERN ${ }^{[20]}$ stammt, da dort die Gesamtgoldkonzentrationen mit der Neutronenaktivierungsanalyse bestimmt wurde, die sich nach Diffusion von Gold in versetzungshaltiges Silizium bei unterschiedlichen Temperaturen und nach verschiedenen Zeiten eingestellt hat. Damit wird in einem im Kap. 5.3 vorgestellten Modell eine Bindungsenergie von Gold an Versetzungen bestimmt. Diese Arbeit trägt einen wichtigen Beitrag zur Interpretation der hier erzielten Ergebnisse bei.

Danach werden die Ergebnisse der DLTS-Messungen sowie die aus den numerischen Anpassungen an die Spektren gewonnenen Parameter erläutert. Gemäß der Beschreibung in Kap. 2.1.2.1 werden für die Modellierung von Defektparametern mittels numerischer Anpassungen sowohl Spektren aus der Frequenz- als auch aus der Pulslängenvariation benötigt. Die Spektren der Frequenzvariation wurden alle bei einer Pulslänge von $100 \mu \mathrm{s}$ und die Spektren der Pulslängenvaration bei einer Pulswiederholfrequenz von $17 \mathrm{~Hz}$ gemessen. Mit der Lage einer DLTS-Linie auf der Temperaturskala ist im folgenden die Temperatur des Maximums bei dieser Pulswiederholfrequenz gemeint.

Im letzten Abschnitt werden Ergebnisse der Transmissionselektronenmikroskopie (TEM) erläutert, mit der wie in der Arbeit von Rodriguez und Mitarbeiter [20] Goldausscheidungen in einer versetzungshaltigen Probe nachgewiesen wurden. Die bei der TEM eingesetzten Standardverfahren sind in der Literatur eingehend beschrieben ${ }^{[65-68]}$. Bei der Analyse der mit der TEM gefundenen Partikel kam die energiedispersive Röntgenspektroskopie (EDX) zum Einsatz, die bei RudOLF ${ }^{[4]}$ beschrieben ist.

\subsection{Versetzungsdichten}

Mit einem Rasterelektronenmikroskop (REM) wurden Aufnahmen mit 8000 facher Vergrößerung aufgenommen. Die Orte der Aufnahmen wurden, wenn nicht anders beschrieben, zufällig ausgewählt. Die Versetzungsdichten wurden durch Zählen von Ätzgrübchen auf einer bestimmten Fläche ermittelt, die, wie in Kap. 3.7 beschrieben, erzeugt wurden. 


\subsubsection{DLTS-Proben}

Abb. 4.1 zeigt exemplarisch eine REM-Abbildung der n-dotieren Probe, die mit 23MPa Belastung verformt wurde.

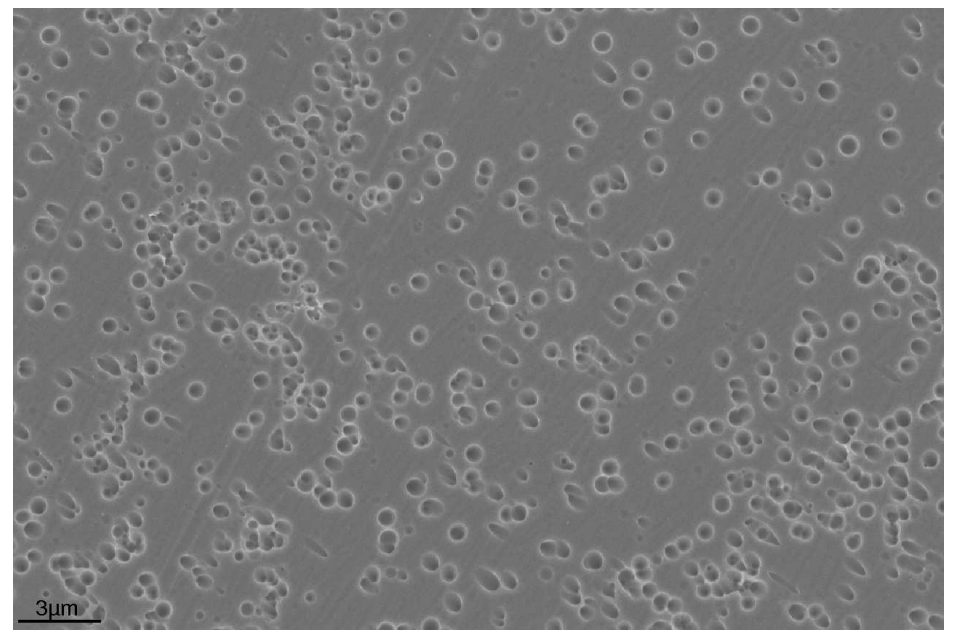

Abbildung 4.1 - REM-Abbildung der defektgeätzten Fläche der n-dotierten Probe, die mit 23MPa Belastung verformt wurde.

Von dieser Probe wurde eine Gesamtfläche von $2488 \mu \mathrm{m}^{2}$ auf vier Abbildungen ausgezählt und eine Versetzungsdichte von $1.4(3) \cdot 10^{8} \mathrm{~cm}^{-2}$ ermittelt, was mit der rechnerisch ermittelten Versetzungsdichte von $1.6 \cdot 10^{8} \mathrm{~cm}^{-2}$ gut übereinstimmt.

Abb. 4.2 zeigt eine REM-Aufnahme der n-dotierten Probe, die mit 34MPA belastet wurde. Die ausgewertete Gesamtfläche betrug hier $2488 \mu \mathrm{m}^{2}$ auf vier Abbildungen. Die Versetzungsdichte wurde zu $3.3(2) \cdot 10^{8} \mathrm{~cm}^{-2}$ bestimmt. Diese stimmt gut mit der rechnerisch ermittelten Versetzungsdichte von $3.5 \cdot 10^{8} \mathrm{~cm}^{-2}$ überein. Da die p-dotierte DLTS-Probe in der gleichen Weise verformt wurde, kann auch hier die gleiche Versetzungsdichte angenommen werden.

\subsubsection{Die unterschiedlich ausgelagerten Proben}

Die Ergebnisse der Bestimmung von Versetzungsdichten der unterschiedlich ausgelagerten Proben finden sich in der Tab. 4.1. Aus den Ergebnissen geht hervor, dass die Versetzungsdichte bei diesen Experimenten bis zu einer Temperatur von $1000^{\circ} \mathrm{C}$ konstant blieben, unabhängig von der Dauer der Auslagerungen. Die Glühung bei $1100^{\circ} \mathrm{C}$ verringerte die Versetzungsdichte signifikant auf etwa $1 / 4$ der ursprünglichen Versetzungsdichte. 


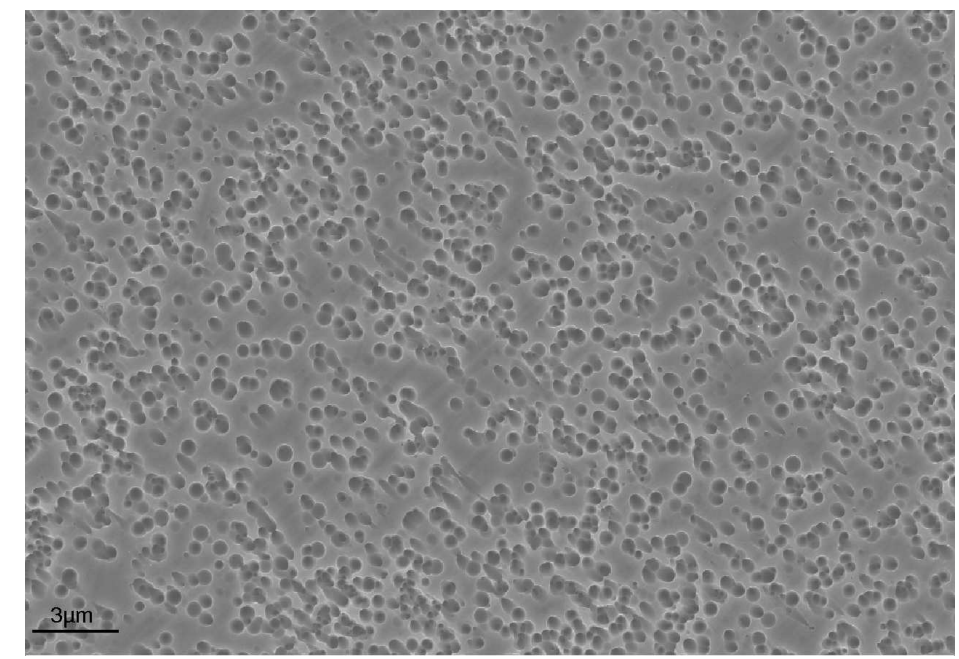

Abbildung 4.2 - REM-Abbildung der defektgeätzten Fläche der n-dotierten Probe, die mit 34MPa Belastung verformt wurde.

\begin{tabular}{|c|c|c|c|}
\hline$T\left({ }^{\circ} \mathrm{C}\right)$ & $t$ & $N_{\mathrm{d}}\left(10^{8} \mathrm{~cm}^{-2}\right)$ & $A\left(\mu \mathrm{m}^{2}\right)$ \\
\hline- & - & $3.3(1)$ & 1866 \\
\hline 800 & $17 \mathrm{~h}$ & $3.1(4)$ & 1866 \\
\hline 900 & $1 \mathrm{~h}$ & $3.1(4)$ & 1866 \\
\hline 1000 & $37 \mathrm{~min}$ & $3.3(2)$ & 1866 \\
\hline 1100 & $27 \mathrm{~min}$ & $0.9(2)$ & 3110 \\
\hline
\end{tabular}

Tabelle 4.1 - Versetzungsdichten $N_{\mathrm{d}}$ in den Proben, die unterschiedlich ausgelagert wurden. $T$ ist die Auslagerungstemperatur, $t$ deren Dauer und $A$ die gesamte ausgewertete Fläche.

\subsubsection{Versetzungsdichte der Probe einer anderen Veröffentlichung}

Für eine Probe aus der Arbeit von Rodriguez und Mitarbeitern ${ }^{[20]}$ wurde mit der Neutronenaktivierungsanalyse (NAA) die Gesamtkonzentration von Gold gemessen, das bei unterschiedlichen Temperaturen für unterschiedliche Zeiten in versetzungshaltige Siliziumproben eindiffundiert worden war. Die Bedingungen, unter denen die Versetzungen erzeugt wurden, stimmten in der Verformungsgeometrie mit der in dieser Arbeit angewendeten Geometrie überein. Die Verformungstemperatur betrug $870^{\circ} \mathrm{C}$. Die Versetzungsdichte, die durch das Zählen von Ätzgrübchen ermittelt wurde, wird mit mindestens $10^{8} \mathrm{~cm}^{-2}$ angegeben.

Die dort gezeigten Diffusionssprofile (siehe Kap. 5.3) zeigen für Temperaturen unter $1000^{\circ} \mathrm{C}$ eine deutliche Erhöhung der Goldkonzentration im Vergleich zu solchen, die durch das Modell von StolwiJk und Mitarbeitern ${ }^{[30]}$ (siehe Kap. 2.2.1) berechnet 
werden können.

Die mit der NAA gemessenen Gesamtkonzentration liefert einen wichtigen Beitrag zur Interpretation der in dieser Arbeit erstellten Ergebnisse. Aufgrund der Möglichkeit, dass die Versetzungsdichte einen Einfluss auf die Gesamtlöslichkeit von Gold in Silizium besitzt, wurde anhand einer verformten Probe, die aus den Experimenten zu dieser Veröffentlichung stammt, mit der Rastertransmissionselektronenmikroskopie (STEM) $\underline{66}, \underline{68}]$ die Versetzungsdichte bestimmt. Dazu wurden vier Aufnahmen eines zusammenhängenden, zufällig ausgesuchten Gebietes mit einer gesamten abgebildeten Fläche von $165 \mu \mathrm{m}^{2}$ untersucht (Abb. 4.3). Durch die Bestimmung der Zonenachse konnte die abgebildete

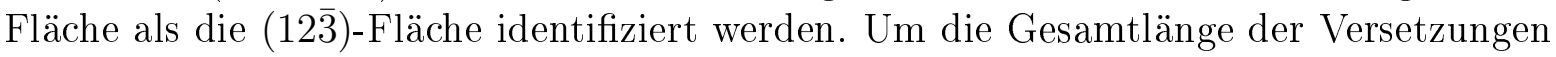

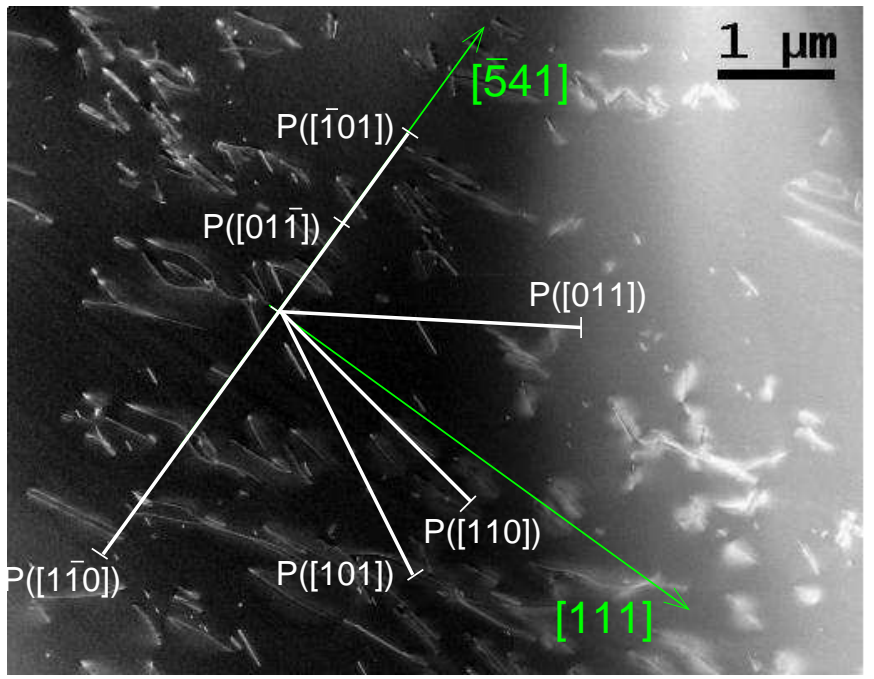

Abbildung 4.3 - STEM-Aufnahme der Probe von Rodriguez und Mitarbeitern zur Bestimmung der Versetzungsdichte. Mit dem Koordinatensystem und den darin eingezeichneten Projektionen der $\langle 110\rangle$-Richtungen $P(\langle 110\rangle)$ konnten die Versetzungen den entsprechenden Richtungen zugeordnet werden.

bestimmen zu können, musste berücksichtigt werden, dass die Versetzungen entlang unterschiedlicher $\langle 110\rangle$-Richtungen liegen und somit unterschiedliche Längen in der Projektion auf die abgebildete Fläche besitzen. Daher wurde in das in Abb. 4.3 gezeigte

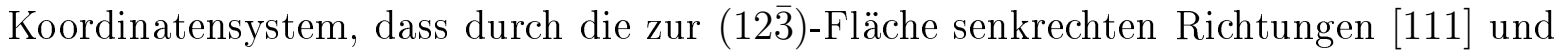
[541] aufgespannt wird, die Projektionen der $\langle 110\rangle$-Richtungen $(\mathrm{P}(\langle 110\rangle))$ längentreu aufgetragen. Auf diese Weise konnten die Versetzungen den Richtungen zugeordnet und deren projizierte Länge mit dem entsprechenden Faktor, der sich aus dem Schnittwinkel

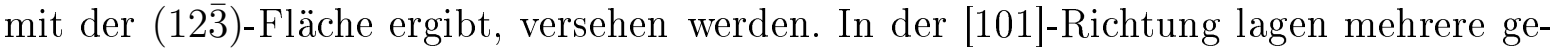
radlinige und Versetzungen und Versetzungen gleicher Länge. Dies deutete darauf hin, dass diese Versetzungen die Probe auf ihrer gesamten Dicke durchzogen. Die Länge dieser Versetzungen konnte herangezogen werden, um die Dicke der Probe zu $0.18 \mu \mathrm{m}$ zu bestimmen, was gut mit der aus den Abbildungsbedingungen abgeschätzten Dicke von 
$0.2 \mu \mathrm{m}$ übereinstimmte. Mit der Gesamtlänge der Versetzungen, die die winkelabhängigen Faktoren berücksichtigt, und der Gesamtfläche sowie der Probendicke konnte die Versetzungsdichte $\mathrm{zu} 8(3) \cdot 10^{8} \mathrm{~cm}^{-2}$ berechnet werden.

\subsection{Ergebnisse der DLTS-Messungen}

\subsubsection{Gold in versetzungsfreiem Silizium}

Wie in Kap. 2.2.3 dargestellt, besitzt substitutionelles Gold zwei tiefe Zustände in der Siliziumbandlücke. Darüber hinaus bildet es mit Wasserstoff, der durch chemische Ätzschritte während der Schottky-Kontaktpräparation eingebracht werden kann, Komplexe, die ebenso mehrere tiefe Zustände in die Bandlücke einführen. Diese Zustände sind auch in golddotiertem versetzungshaltigen Silizium zu erwarten. Die in diesem Abschnitt ermittelten Parameter der goldinduzierten Zustände (Energieniveaus, Einfangquerschnitte, Einfangkinetiken, Emissionscharakteristiken) in versetzungsfreiem Silizium dienen später in der Diskussion der Identifizierung versetzungsbedingter Einflüsse auf die DLTSSpektren versetzungshaltiger Proben.

\subsubsection{1 n-dotiertes Silizium}

Die an der n-dotierten Probe mit der Sperrspannung $U_{\mathrm{b}}=-5 \mathrm{~V}$ und der Pulsspannung $U_{\mathrm{P}}=4.9 \mathrm{~V}$ gemessenen DLTS-Spektren sind in der Abbildung 4.4(a) gezeigt. Die Spektren enthalten zwei dominierende Linien, die in dem gleichen System schon in anderen Arbeiten gemessen wurden ${ }^{[42,43,69]}$. Gemäß der dort verwendeten Kennzeichnungen wird die Linie, die bei ca. 103K liegt, mit G1, und die, die bei ca. 264K liegt, mit Au+G4 bezeichnet. G1 wird in den referenzierten Arbeiten dem Doppelakzeptorzustand des Gold-Wasserstoff-Komlexes $\mathrm{AuH}^{(-/-)}$zugeordnet, während $\mathrm{Au}+\mathrm{G} 4$ aus einer Überlagerung zweier DLTS-Linien resultiert, die von dem Goldakzeptorzustand $\mathrm{Au}^{(0 /-)}$ bzw. dem Akzeptorzustand des Gold-Wasserstoff-Komplexes $\mathrm{AuH}^{(0 /-)}$ herrühren (vgl. Kap. 2.2.3).

Die Abb. 4.4(b) zeigt die Spektren, die an den gleichen Kontakten gemessen wurden, nachdem die Probe für eine halbe Stunde bei $250^{\circ} \mathrm{C}$ ausgelagert worden ist. Diese Wärmebehandlung ermöglicht laut SveINBJÖRNSSON UND ENGSTRÖM ${ }^{[42]}$ die Auflösung sowohl der AuH als auch weiterer, elektrisch inaktiver Gold-Wasserstoff-Komplexe. Letztere konnten dort indirekt durch Temperaturbehandlungen nachgewiesen werden, die zu Konzentrationsabnahmen sowohl der AuH und der Goldzustände als auch der Paare aus Dotieratomen und Wasserstoff führten. Da außerdem keine neuen elektrisch aktiven Zustände gefunden werden konnte, wurde angenommen, dass die Goldatome passiviert wurden.

Die Wärmebehandlung bewirkte in der vorliegenden Arbeit wie in ${ }^{[42]}$ eine deutliche Reduktion der G1-Amplitude und einen Anstieg sowie eine Verschiebung der $\mathrm{Au}+\mathrm{G} 4-$ Linie um etwa $4 \mathrm{~K}$ zu einer tieferen Temperatur. Mit der Annahme, dass G1 und G4 

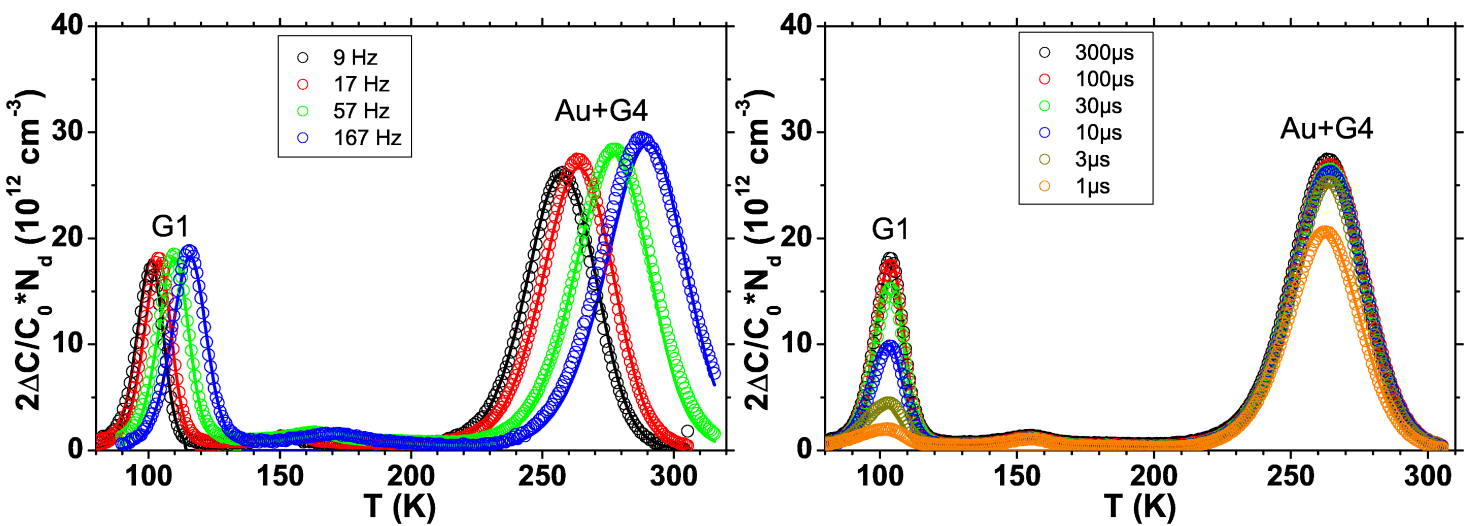

(a) vor dem Auslagern
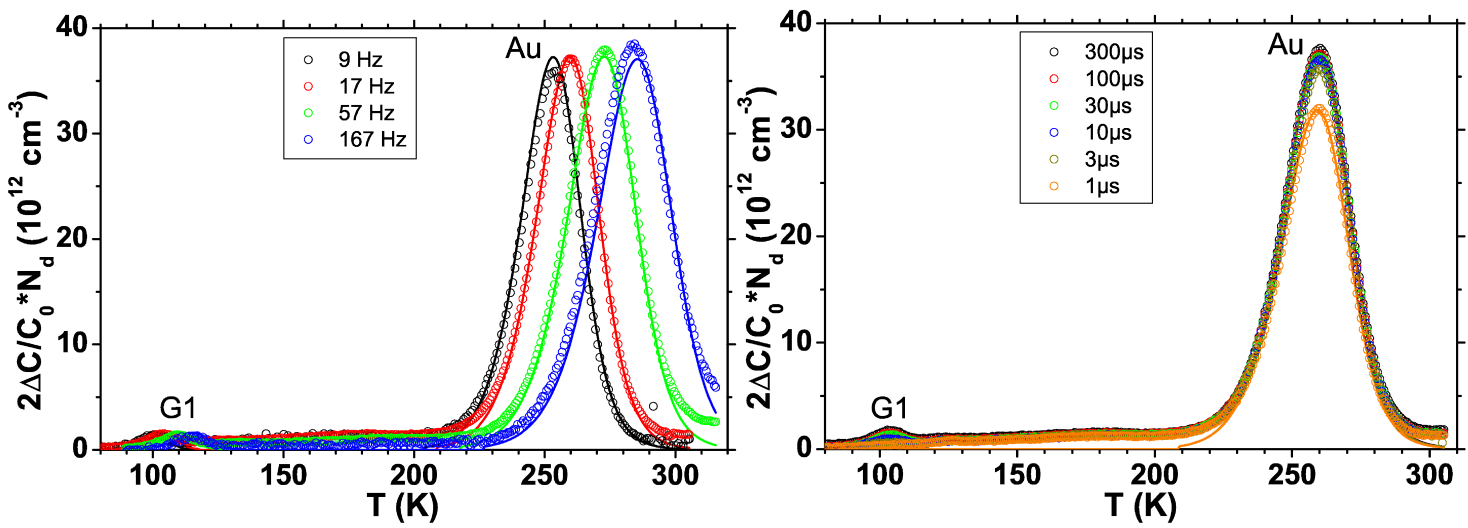

(b) nach dem Auslagern $\left(250^{\circ} \mathrm{C}, 30\right.$ Minuten)

Abbildung 4.4 - DLTS-Spektren mit dem Ergebnis numerischer Anpassung (durchgezogene Linien) der golddotierten versetzungsfreien Probe, $\left(U_{\mathrm{b}}=-5 \mathrm{~V}, U_{\mathrm{P}}=4.9 \mathrm{~V}\right)$, Frequenzvariation, $t_{\mathrm{p}}=100 \mu \mathrm{s}$, Pulslängenvariation, $f_{\mathrm{w}}=17 \mathrm{~Hz}$. a) vor dem Auslagern, b) nach dem Auslagern bei $250^{\circ} \mathrm{C}$ für 30 Minuten.

DLTS-Linien desselben AuH sind, kann geschlossen werden, dass die Linie, die nach der Wärmebehandlung bei $260 \mathrm{~K}$ erscheint, $\mathrm{zu} \mathrm{Au}^{(0 /-)}$ gehört. Für die numerische Anpassung der DLTS-Linien dieses Defektes an die gemessenen Spektren wurden die Parameter Defektkonzentration $N_{\mathrm{T}}$, Aktivierungsenthalpie $\Delta H_{n}$, Einfangquerschnitt der Majoritätsladungsträger $\sigma_{n}$ und Entropiefaktor $\chi_{n}$ variiert. Aus den Ergebnissen für zwei unterschiedliche Kontakte wurde ein Mittelwert und die in der Tab. 4.2 angegebenen Fehler berechnet. Für die Bestimmung des Vorfaktors der Minoritätsladungsträgeremissionsrate $\sigma_{p} \chi_{p}$, deren Werte in dieser Messung nicht separat bestimmt werden können (siehe Kap. 2.1.1), wurde $\chi_{p}$ in der Anpassung auf den Wert eins fixiert. Das Anpassungsergebnis für $\sigma_{p}$ waren Werte, die um mehrere Größenordnungen kleiner Waren als $\sigma_{n}$. Somit wurde diese Größe während weiterer Anpassungen in den n-dotierten Proben auf einen vernachlässigbar kleinen Wert gehalten $\left(10^{-19} \mathrm{~cm}^{2}\right)$. Die in Klammern angegeben Werte 


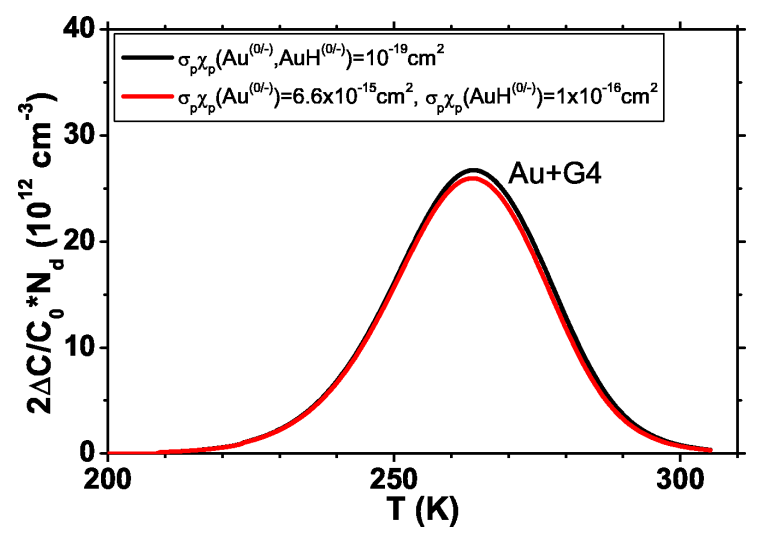

Abbildung 4.5 - Ergebnis der numerischen Anpassung unter Vernachlässigung der Minoritätsladungsträgeremission (schwarz) und mit der Minoritätsladungsträgeremission aus den Ergebnissen der p-dotierten Probe (rot). Diese Neuberechnung führt zu einer geringen Korrektur der Linienamplitude und zu einer leichten Verschiebung des Linienmaximums um $0.3 \mathrm{~K}$ zu tieferen Temperaturen.

entstammen den Ergebnissen der p-dotierten Proben (Kap. 4.2.1.2), wo dieser Zustand aufgrund seiner Lage in der Mitte der Bandlücke ebenfalls gemessen wird. Diese Werte führten in der Neuberechnung mit den ursprünglichen Ergebnissen der Anpassung zu kleinen Korrekturen in der Dichte der $\mathrm{Au}^{(0 /-)}\left(2.9 \cdot 10^{13} \mathrm{~cm}^{-3}\right.$ bzw. $\left.2.7 \cdot 10^{13} \mathrm{~cm}^{-3}\right)(\mathrm{Au})$ und zu einer leichten Verschiebung des Linienmaximums um $0.3 \mathrm{~K}$ zu niedrigeren Temperaturen. Abb. 4.5 zeigt die für $t_{\mathrm{p}}=300 \mu$ s berechneten Spektren der Linie $\mathrm{Au}+\mathrm{G} 4$ vor der Neuberechnung, d.h. vernachlässigter Minoritätsladungsträgeremission (schwarz), und nach der Neuberechnung mit den Ergebnissen aus den p-dotierten Proben (rot).

In den Diagrammen der Abb. 4.4 sind die Ergebnisse der Anpassungen mit durchgezogenen Linien dargestellt. Die dabei errechneten Werte für $\Delta H, \sigma_{n}$ und $\chi_{n}$ des $\mathrm{Au}^{(0 /-)}$ wurden dann benutzt, um die entsprechenden Parameter des $\mathrm{AuH}^{(0 /-)}$ durch numerische Anpassung an die Linie $\mathrm{Au}+\mathrm{G} 4 \mathrm{zu}$ berechnen. Hier wurde ausgenutzt, dass die Konzentration der $\mathrm{AuH}$ durch Anpassung der $\mathrm{AuH}^{(-/-)}$-Parameter an die G1-Linie berechnet werden konnte, so dass als Anpassungsparameter für $\mathrm{AuH}^{(0 /-)}$ nur $\Delta H, \sigma_{n}$ und $\chi_{n}$ variiert werden mussten. Das Ergebnis für $\sigma_{p} \chi_{p}$ wurde in gleicher Weise erzielt wie bei $\mathrm{Au}^{(0 /-)}$. Die auf diese Weise berechneten Größen sind in der Tab. $4.2 \mathrm{im}$ Vergleich mit denen anderer Arbeiten zusammengefasst.

Zum Vergleich der Emissionscharakteristiken von $\mathrm{Au}^{(0 /-)}$ und $\mathrm{AuH}^{(0 /-)}$ sind die in dieser Arbeit ermittelten Werte in der Abb. 4.6 als Arrhenius-Auftragung mit denen von Rubaldo ET AL. ${ }^{43]}$ dargestellt. Die in Tab. 4.2 gezeigten Entropiefaktoren wurden aus den durch lineare Regression errechneten Ordinatenabschnitten bestimmt (vgl. Kap. 2.1.1) und daher in Klammern geschrieben. Im untersuchten Temperaturbereich gibt es eine gute Übereinstimmung der jeweils bestimmten Emissionscharakteristiken. In den

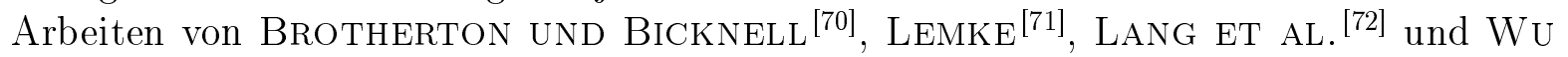


UND PEAKER ${ }^{[73]}$ wurde nicht berücksichtigt, dass es sich bei der Linie, die der Emission aus $\mathrm{Au}^{(0 /-)}$ zugeordnet wurde, wahrscheinlich um die Überlagerung zweier DLTS-Linien handelt. Somit entstammen die Daten der entsprechenden Auftragungen einer Überlagerung der Emissionseigenschaften der zur $\mathrm{Au}+\mathrm{G} 4$-Linie gehörenden Defekte. Dabei

\begin{tabular}{|c|c|c|c|c|}
\hline & & $\mathbf{A u H}(-/--)$ & $\mathbf{A} u^{(0 /-)}$ & $\mathbf{A u H}(0 /-)$ \\
\hline \multirow{4}{*}{ diese Arbeit } & $\Delta H(\mathrm{eV})$ & $0.18165(9)$ & $0.513(1)$ & $0.604(1)$ \\
\hline & $\sigma_{n}\left(\mathrm{~cm}^{2}\right)$ & $6.87(5) \cdot 10^{-18}$ & $1.188(6) \cdot 10^{-16}$ & $4.9(7) \cdot 10^{-17}$ \\
\hline & $\chi_{n}$ & $56(8)$ & $7(1)$ & $278(69)$ \\
\hline & $\sigma_{p} \chi_{p}\left(\mathrm{~cm}^{2}\right)$ & & $\left(6.6 \cdot 10^{-15}\right)$ & $\left(1.0 \cdot 10^{-16}\right)$ \\
\hline \multirow{3}{*}{ [42] } & $\Delta H(\mathrm{eV})$ & 0.19 & & \\
\hline & $\sigma_{n}\left(\mathrm{~cm}^{2}\right)$ & $1 \cdot 10^{-17}$ & & \\
\hline & $\chi_{n}$ & & & \\
\hline \multirow{3}{*}{ [43] } & $\overline{\Delta H(\mathrm{eV})}$ & $0.193 \pm 3$ & $0.558 \pm 8$ & $0.542 \pm 8$ \\
\hline & $\sigma_{n}\left(\mathrm{~cm}^{2}\right)$ & & $1 \cdot 10^{-16}$ & $6 \cdot 10^{-17}$ \\
\hline & $\chi_{n}$ & & $(54.3)$ & $(19.1)$ \\
\hline
\end{tabular}

Tabelle 4.2 - Durch numerische Anpassung gewonnene Parameter des Goldakzeptors $A u^{(0 /-)}$, des Gold-Wasserstoffakzeptors $A u H^{(0 /-)}$ und des Gold-Wasserstoffdoppelakzeptors $A u H^{(-/-)}$im Vergleich mit den Daten von SveinBjöRnsson und EngSTRöm ${ }^{[42]}$ und Rubaldo und Mitarbeiter $\stackrel{[43]}{\text {. }}$.

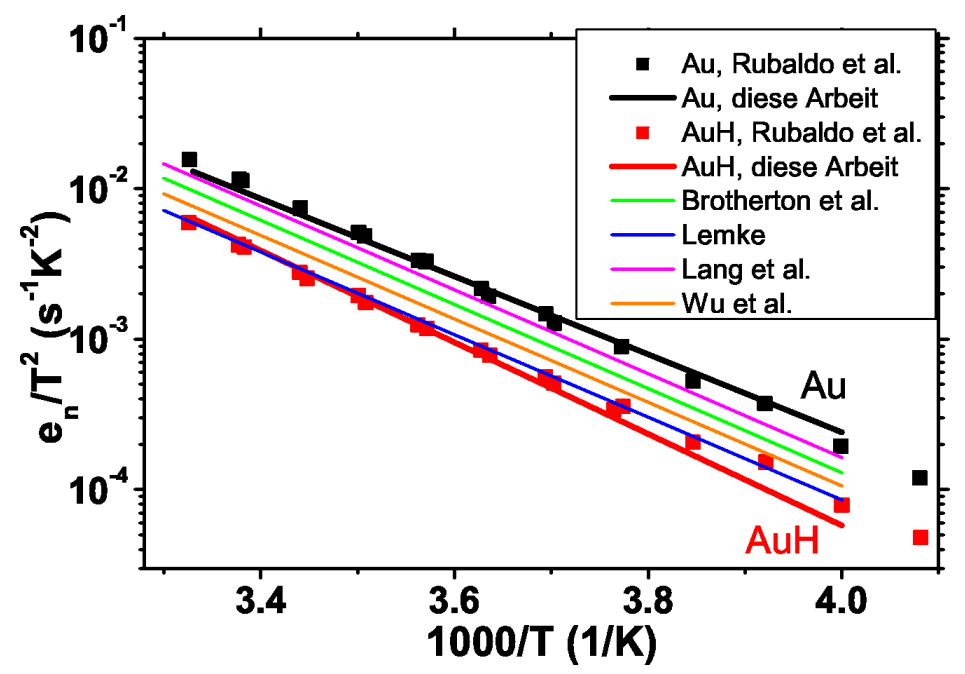

Abbildung 4.6 - Thermische Emissionsraten: Vergleich der Ergebnisse der numerischen Anpassung

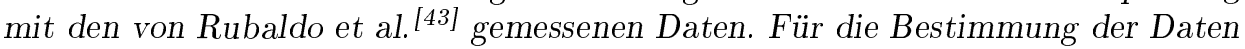
von $\underline{\underline{70}-\underline{\underline{7}}]}$ wurde keine Überlagerung zweier DLTS-Linien berücksichtigt. 
können die Beiträge der beiden Einzellinien je nach Konzentration der beteiligten Defekte variieren.

\subsubsection{2 p-dotiertes Silizium}

Wie in Kap. 2.2.3 beschrieben, finden sich im DLTS-Spektrum von p-dotiertem Silizium, in das Gold eindiffundiert wurde, neben der Linie des Golddonatorzustands $\mathrm{Au}^{(+/ 0)}$ laut SveInBJÖRnsson Und ENGSTRÖM ${ }^{[42]}$ auch Linien, die Komplexen aus Gold und Wasserstoff zugeschrieben werden können. In Abb. 4.7 sind DLTS-Spektren einer versetzungsfreien p-dotierten Probe zusammen mit dem Ergebnis der numerischen Anpassung (durchgezogene Linien) abgebildet. Diese Probe wurde im Rahmen der SchottkyKontaktpräparation zwischen dem HF-Dip und dem Einbau in die Aufdampfanlage für 20 Minuten unter eine Halogenlampe gelegt (siehe Kap. 3.6). Eine kontrollierte Lagerung nach dem Ätzen mit $\mathrm{HF}: \mathrm{H}_{2} \mathrm{O}$ ist notwendig, um Siliziumoxid als isolierende Schicht zum Metall wachsen zu lassen (siehe Kap. 3.6). Die Linie bei 108K entspricht der Linie G2 und
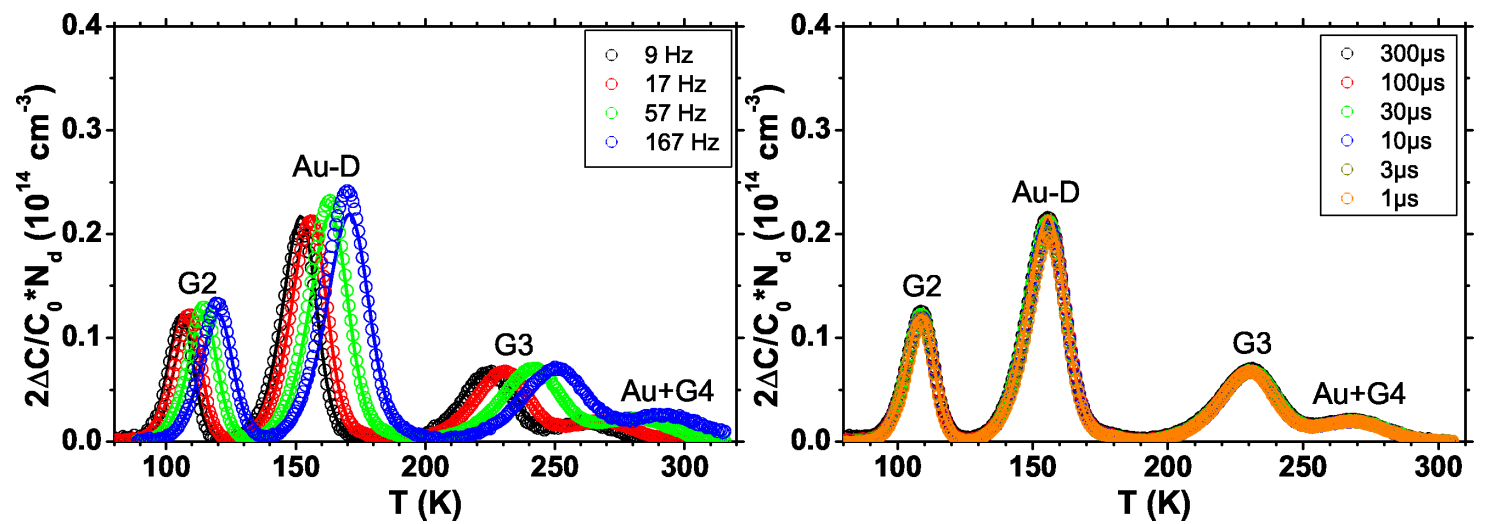

Abbildung 4.7 - DLTS-Spektren mit dem Ergebnis numerischer Anpassung (durchgezogene Linien) der golddotierten versetzungsfreien Probe $\left(U_{\mathrm{b}}=-5 \mathrm{~V}, U_{\mathrm{P}}=4.9 \mathrm{~V}\right.$, Frequenzvariation, $t_{\mathrm{p}}=100 \mu \mathrm{s}$, Pulslängenvariation, $\left.f_{\mathrm{w}}=17 \mathrm{~Hz}\right)$. Vor dem Aufdampfen der Aluminiumkontakte wurde diese Probe für 20 Minuten unter einer Halogenlampe gelagert.

ist dem Donatorzustand des Gold-Wasserstoff-Komplexes AuH ${ }^{(+/ 0)}$ zugeordnet, während die Linie bei $155 \mathrm{~K}$ zum Golddonatorzustand $\mathrm{Au}^{(+/ 0)}$ gehört. Aufgrund der Eigenschaften, die im Zusammenhang mit Auslagerungsexperimenten bei der Linie G3 beobachtet wurden, wurde diese Linie, die im Spektrum bei 230K liegt, mit einem wasserstoffbedingten Defekt, nicht jedoch mit dem AuH assoziiert. Im oberen Temperaturbereich liegt eine DLTS-Linie bei $268 \mathrm{~K}$, die einer Überlagerung der Linien des $\mathrm{Au}^{(0 /-)}$ - und des $\mathrm{AuH}^{(0 /-)}$-Akzeptorzustandes zugeordnet wird $(\mathrm{Au}+\mathrm{G} 4)$, die im p-dotierten Silizium aufgrund ihrer Lage nahe der Bandlückenmitte gemessen werden. Die zugehörigen Zustände emittieren in n-dotierten Silizium in das Leitungsband und im p-dotiertem Silizium in das Valenzband. In der numerischen Anpassung wurden daher die Energieniveaus dieser 
Zustände, die in der Tab. 4.3 eingeklammert sind, jeweils auf die Differenz der Bandlückenenergie $\left(E_{\mathrm{g}}=1.12 \mathrm{eV}\right)$ und der Energien festgelegt, die für diese Defekte mit der numerischen Anpassung der Spektren der n-dotierten Probe ermittelt wurden. Außerdem wurde die Defektkonzentration von $\mathrm{AuH}^{(0 /-)}$ auf die Konzentration von $\mathrm{AuH}^{(+/ 0)}$ und die von $\mathrm{Au}^{(0 /-)}$ auf die Konzentration von $\mathrm{Au}^{(+/ 0)}$ festgelegt, da es sich bei diesen um unterschiedliche Zustände des jeweils gleichen Defektes handelt. Somit waren als Anpassungsparameter für diese Linie nur die Vorfaktoren $\sigma_{p} \chi_{p}$ nötig, da die Einfangquerschnitte $\sigma_{n}$ und Entropiefaktoren $\chi_{n}$ ebenfalls aus den Ergebnissen der n-dotierten Probe bekannt waren.

In der Abb. 4.8 sind die Spektren eines Kontaktes der Probe dargestellt, die nach dem Ätzen durch den HF-Dip nicht unter einer Halogenlampe gelegt, sondern bei Raumtemperatur für die gleiche Dauer gelagert wurde.

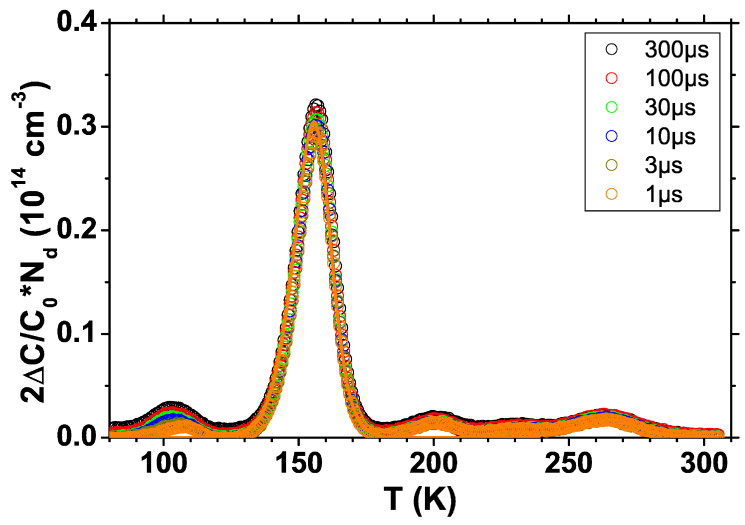

Abbildung 4.8 - DLTS-Spektren mit dem Ergebnis numerischer Anpassung (durchgezogene Linien) der golddotierten versetzungsfreien Probe $\left(U_{\mathrm{b}}=-5 \mathrm{~V}, U_{\mathrm{P}}=4.9 \mathrm{~V}\right.$, Pulslängenvariation, $\left.f_{\mathrm{w}}=17 \mathrm{~Hz}\right)$. Diese Probe wurde vor dem Aufdampfen der Aluminiumkontakte für 20 Minuten bei Raumtemperatur gelagert.

Im Vergleich mit den in Abb. 4.7 dargestellten Spektren der oben beschriebenen Probe (,mit Halogenlicht“) fällt auf, dass die Amplituden der Linien, die den Gold-WasserstoffKomplexen zugeordnet werden (G2, G3), kleiner ausfallen im Vergleich mit der Linie des Golddonators $\mathrm{Au}^{(+/ 0)}$. Außerdem ist die Linie um 105K im Vergleich zu G2 zu tieferen Temperaturen verschoben. Es scheint, dass es sich bei dieser Linie um eine Überlagerung zweier Linien handelt, was an der Verschiebung der Maximumtemperatur für unterschiedliche Einfangszeiten $t_{\mathrm{p}}$ deutlich wird. Entsprechendes gilt für die Linie G3, wohingegen die Linie des Golddonators als unverändert in den Emissionseigenschaften beobachtet wird, da hier die Anpassung mit den gleichen Parametern wie die der oben beschriebenen Probe zu gleichen Ergebnissen führt. Die Linie im oberen Temperaturbereich bei 265K $(\mathrm{Au}+\mathrm{G} 4)$, die den Akzeptorzuständen des Goldes und des Gold-WasserstoffKomplexes zugeschrieben wird, ist im Vergleich zu der oben beschriebenen Probe zu tieferen Temperaturen verschoben. Wie bei SvEINBJÖRnsSON UND ENGSTRÖM ${ }^{[42]}$ geht 
eine Abnahme der G2-Amplitude mit dieser Verschiebung von $\mathrm{Au}+\mathrm{G} 4$ einher. Diese Effekte werden dort mit dem Zerstören der Gold-Wasserstoff-Komplexe erklärt, was demzufolge zu einer Reduktion der G2- und der G4-Amplitude führt. Tabelle 4.3 zeigt die Ergebnisse aus der numerischen Anpassung der Spektren der p-dotierten Proben. Da die

\begin{tabular}{|l|c||c|c|c|c|c|}
\cline { 3 - 7 } \multicolumn{2}{c|}{} & $\mathbf{A u H}$ & & \\
\hline \multirow{4}{*}{$\begin{array}{l}\text { diese } \\
\text { Arbeit }\end{array}$} & $\Delta H(\mathrm{eV})$ & $0.20837(2)$ & $0.3209(3)$ & $0.4676(2)$ & $(0.516)$ & $(0.607)$ \\
\cline { 2 - 7 } & $\sigma_{p} \chi_{p}\left(\mathrm{~cm}^{2}\right)$ & $8(1) \cdot 10^{-15}$ & $2.1(2) \cdot 10^{-14}$ & $7(3) \cdot 10^{-15}$ & $1.0(3) \cdot 10^{-16}$ & $6.6(1) \cdot 10^{-15}$ \\
\cline { 2 - 7 } & $\sigma_{n}\left(\mathrm{~cm}^{2}\right)$ & & & & $\left(4.9 \cdot 10^{-17}\right)$ & $\left(1.188 \cdot 10^{-16}\right)$ \\
\cline { 2 - 7 } & $\chi_{n}$ & & & & $(278)$ & $(7)$ \\
\hline \multirow{3}{*}[\mathbf{42}]{} & $\Delta H(\mathrm{eV})$ & 0.21 & 0.35 & 0.47 & \multicolumn{2}{|c|}{$\approx 0.53$} \\
\cline { 2 - 7 } & $\sigma_{p} \chi_{p}\left(\mathrm{~cm}^{2}\right)$ & $1.7 \cdot 10^{-15}$ & $1.0 \cdot 10^{-15}$ & $5.0 \cdot 10^{-16}$ & & \\
\hline
\end{tabular}

Tabelle 4.3 - Durch numerische Anpassung gewonnene Parameter des Golddonators $\mathrm{Au}^{(+/ 0)}$, des Gold-Wasserstoffdonators $A u H^{(+/ 0)}$, eines weiteren wasserstoffinduzierten Defektes (G3) und des Goldakzeptors $\mathrm{Au}^{(0 /-)}$ sowie des Gold-Wasserstoffakzeptors $A u H^{(0 /-)}$ im Vergleich mit den Daten von Sveinbjörnsson und Engström [42]. Die Energieniveaus der beiden Akzeptorzustände wurden aus den Ergebnissen der n-dotierten Proben übernommen und waren daher keine Anpassungsparameter.

Variation der Linienamplituden unter der Variation der Einfangzeit klein im Vergleich zur Amplitude selbst ist, wurden die Werte der Entropiefaktoren der Majoritätsladungsträger in der numerischen Anpassung auf eins fixiert. Somit diente hier der Vorfaktor $\sigma_{p} \chi_{p}$ als Anpassungsparameter. Um diese Werte nicht nur als Produkt sondern getrennt voneinander genau bestimmen zu können, ist eine deutlichere Dynamik in der Änderung der Amplituden unter der Pulslängenvariation erforderlich (siehe Kap. 2.1.1). Dieser Vorfaktor ist als Teil der Emissionscharakteristik unabhängig von der Einfangcharakteristik und damit zuverlässig bestimmt. Die verminderte Amplitude der Linie der Akzeptorzustände $(\mathrm{Au}+\mathrm{G} 4)$ im Vergleich zu der der zugehörigen Donatorniveaus (Au-D) ergibt sich aus einem signifikanten Beitrag der Elektronenemission ins Leitungsband aufgrund der Lage in der Mitte der Bandlücke. Ohne Berücksichtigung des Einfangs von Ladungsträgern aus dem Debye-Tail (siehe Anhang $\mathrm{A}$ ) ergibt sich mit der Gleichgewichtsbedingung $(t \rightarrow \infty)$ aus den Gleichungen (2.1) und (2.2) der Zusammenhang

$$
\begin{aligned}
\frac{N_{T}^{-}}{N_{T}} & =\frac{e_{p}}{e_{p}+e_{n}} \\
& =\frac{1}{1+\frac{N_{\mathrm{L}} \sigma_{n} \chi_{n}}{N_{\mathrm{V}} \sigma_{p} \chi_{p}} \exp \left(\frac{2 \Delta H_{p}-E_{\mathrm{g}}}{k_{\mathrm{B}} T}\right)}
\end{aligned}
$$

für das Verhältnis aus umgeladenen zu vorhandenen Akzeptoren $\mathrm{Au}^{(0 /-)}$. UTzIG ${ }^{[74]}$ be- 
rechnet dieses Verhältnis für den Fall, dass der Beitrag des $\mathrm{AuH}^{(0 /-)}$-Akzeptorzustandes zur Linie $\mathrm{Au}+\mathrm{G} 4$ unberücksichtigt bleibt und diese DLTS-Linie nur dem $\mathrm{Au}^{(0 /-)_{-}}$ Akzeptorzustand zugeordnet wird, $\mathrm{zu} \frac{1}{19.8}$. Mit der in Abb. (4.7) abgelesenen Temperatur des Linienmaximums von $\mathrm{Au}(265 \mathrm{~K})$ berechnet sich dieses Verhältnis für $\mathrm{Au}^{(0 /-)} \mathrm{zu}$ $\frac{1}{20.8}\left(N_{\mathrm{L}}=5.44 \cdot\left(\frac{T}{\mathrm{~K}}\right)^{3 / 2} \cdot 10^{15} \mathrm{~cm}^{-3}, N_{\mathrm{L}}=2.12 \cdot\left(\frac{T}{\mathrm{~K}}\right)^{3 / 2} \cdot 10^{15} \mathrm{~cm}^{-3[75]}\right)$. Das Verhältnis der Amplituden von Au zu Au-D beträgt $\frac{1}{15.5}$.

In Abb. 4.9 werden die Emissionscharakteristiken der in den p-dotierten Proben gemessenen Defekte mit den Ergebnissen von SvEINBJÖRNSSON UND ENGSTRÖM ${ }^{[42]}$ und LEM$\mathrm{KE}^{[11]}$ verglichen. Der Verlauf der hier gemessenen Emissionscharakteristik des $\mathrm{Au}^{(+/ 0)}$ erscheint im Vergleich zu den anderen Ergebnissen zu tieferen Temperaturen verschoben, während dessen Steigung, also dessen Energieniveau mit den Literaturwerten gut übereinstimmt. Darüber hinaus gibt es gute Übereinstimmungen innerhalb der Verläufe der übrigen Defekte im beobachteten Temperaturbereich. Zusammenfassend kann gesagt

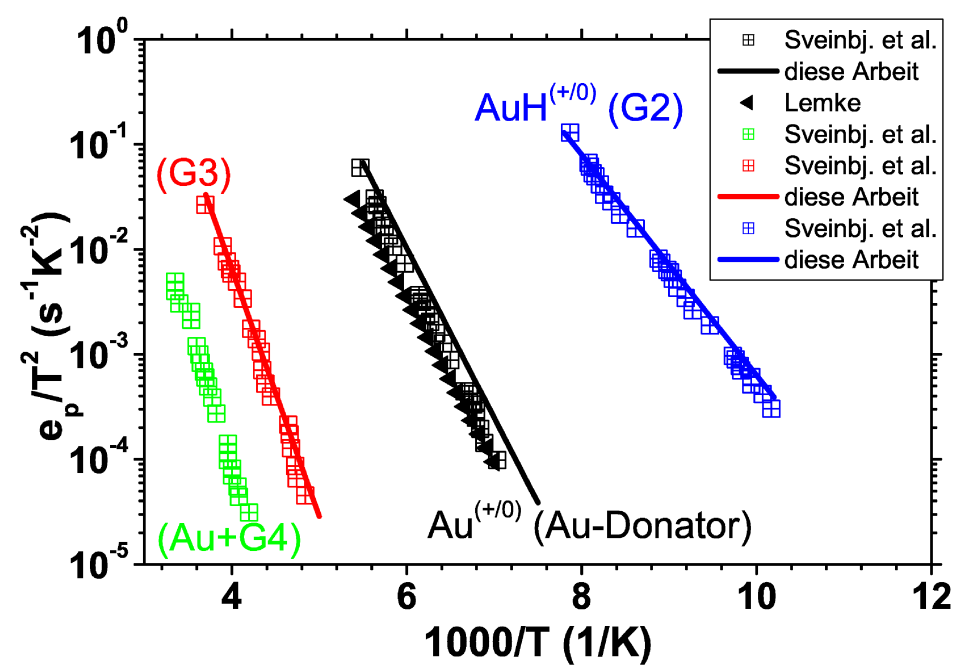

Abbildung 4.9 - Arrhenius-Auftragung der in p-dotiertem Silizium gefundenen thermischen Emissionsraten. Die durchgezogenen Linien wurden aus den in Tab. 4.3 dargestellten Werten berechnet.

werden, dass die hier erzielten Ergebnisse über die Eigenschaften von Gold in versetzungsfreiem Silizium gut mit denen in der Literatur übereinstimmen. Abb. 4.10 zeigt die Lage der goldinduzierten Energieniveaus in einem Bandschema: 


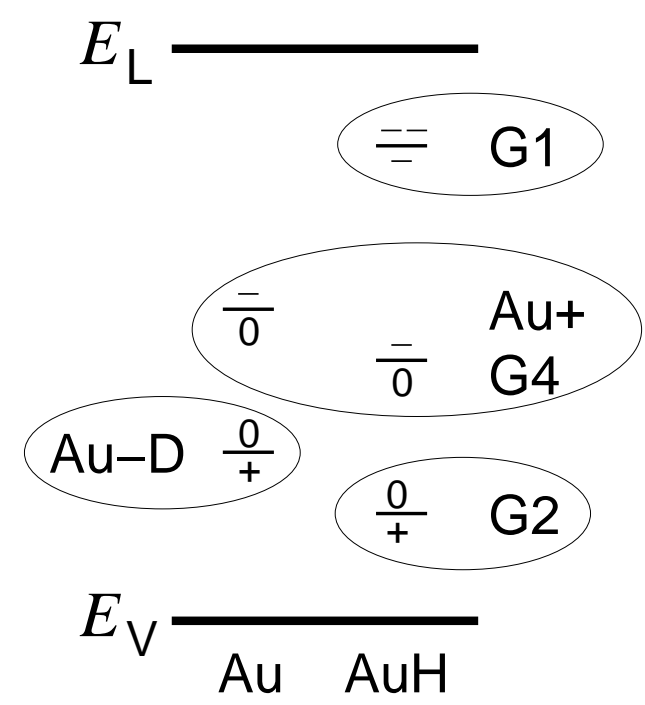

Abbildung 4.10 - Bandschema der goldinduzierten Defekte, $E_{\mathrm{L}}, E_{\mathrm{V}}$ : Leitungs-, Valenzbandkante, $\mathrm{Au}, \mathrm{AuH}$ : substitutionelles Gold und Gold-Wasserstoff-Komplex mit den dazugehörigen Donator- und Akzeptorzuständen. In dieser Abbildung werden die goldinduzierten Zustände den DLTS-Linien zugeordnet. Der DLTS-Linie G4 des Akzeptorzustand des Gold-Wasserstoff-Komplexes $\mathrm{AuH}^{(0 /-)}$ liegt aufgrund des kleinen Einfangquerschnitts im Bereich der Linie Au des Goldakzeptorzustandes $\mathrm{Au}^{(0 /-)}$. Daher können diese Linien nicht getrennt werden. 


\subsubsection{Gold in versetzungshaltigem Silizium}

In diesem Abschnitt werden die Ergebnisse der DLTS-Messungen an golddotierten versetzungshaltigen Proben erläutert. Wie in Kap. 3 erläutert, wurden die Schottky-Kontakte auf einer Fläche präpariert, in der aufgrund eines Schrägschliffs eine laterale Änderung der Goldkonzentration zu erwarten ist. Wenn im folgenden vom Abstand zur Golddiffusionsquelle die Rede ist, dann ist damit der Abstand des entsprechenden Kontaktes zu der Goldschicht gemeint, der durch diesen Schrägschliff entstanden ist (vgl. Abb. 3.2). Für n-dotiertes Silizium mit Versetzungen, in das Gold eindiffundiert wurde, ist nachgewiesen worden, dass die Emissionscharakteristik des zu dem Gold gehörenden tiefen Zustands dem vom Goldakzeptorniveau in versetzungsfreiem Material entspricht [60]. Allerdings zeigte der Elektroneneinfang das Verhalten lokalisierter ausgedehnter Defekte (siehe Kap. 2.1.1). Erklärt wurde dieser Befund mit der Anreicherung von Gold im Verzerrungsfeld der Versetzungen aufgrund der elastischen Wechselwirkung (siehe Kap. 2.3.1.2). Die Goldkonzentration müsste dort so groß sein, dass es zu Überlagerungen der elektrischen Potentiale eingefangener Elektronen und somit zur Ausbildung einer besetzungsabhängigen Einfangbarriere kommt. RODRIGUEZ ET AL. ${ }^{[20]}$ haben die Konzentrationen von Gold, das bei unterschiedlichen Temperaturen in versetzungshaltiges Silizium eindiffundiert wurde, mit der Neutronenaktivierungsanalyse gemessen. Die dort gemessenen Konzentration sind allerdings so groß, dass dies nicht durch eine Anreicherung des Goldes in den Verzerrungsfeldern der Versetzungen erklärt werden kann (siehe Anhang B). Die Ergebnisse dieser Arbeit und deren Analyse werden im Kap. 5 detailliert betrachtet. 


\subsubsection{1 n-dotiertes Silizium}

Um die Wechselwirkungen von Versetzungen und Gold zu untersuchen, wurden Proben mit unterschiedlichen Versetzungsdichten $\left(N_{\mathrm{d}, 1}=1.4 \cdot 10^{8} \mathrm{~cm}^{-2}\right.$ und $N_{\mathrm{d}, 2}=3.3 \cdot 10^{8} \mathrm{~cm}^{-2}$, Kap. 4.1.1) hergestellt. Durch Eindiffusion bei $800^{\circ} \mathrm{C}$ für 17 Stunden wurde in diesen Proben ein Konzentrationsprofil eingestellt, wobei sich zumindest in Abwesenheit Goldausscheidungen innerhalb der Probe an der Oberfläche die Löslichkeit, d.h. die Konzentration im Gleichgewicht mit der flüssigen Phase, einstellt. Durch eine Schrägschlifftechnik konnten Schottky-Kontakte in unterschiedlichen Abständen zur Golddiffusionsquelle aufgebracht werden. Die Kontakte sind mit ihren Bezeichnungen und den Abständen zur Golddiffusionsquelle in der Tabelle 4.4 gezeigt. Somit war es möglich, den Einfluss unterschiedlicher Versetzungsdichten und variierender Goldkonzentrationen auf das DLTSSpektrum zu messen. Für die detaillierte Beschreibung der Probenpräparation sei auf das Kap. 3 verwiesen.

\begin{tabular}{|c|c||c|c|}
\hline \multicolumn{4}{|c|}{ Versetzungsdichte } \\
\hline \multicolumn{2}{|c||}{$3.3 \cdot 10^{8} \mathrm{~cm}^{-2}$} & \multicolumn{2}{|c|}{$1.4(3) \cdot 10^{7} \mathrm{~cm}^{-2}$} \\
\hline$\# 1 \mathrm{a}$ & $37 \mu \mathrm{m}$ & $\# 1 \mathrm{~b}$ & $34 \mu \mathrm{m}$ \\
\hline$\# 2 \mathrm{a}$ & $71 \mu \mathrm{m}$ & $\# 2 \mathrm{~b}$ & $73 \mu \mathrm{m}$ \\
\hline$\# 3 \mathrm{a}$ & $80 \mu \mathrm{m}$ & $\# 3 \mathrm{~b}$ & $115 \mu \mathrm{m}$ \\
\hline$\# 4 \mathrm{a}$ & $88 \mu \mathrm{m}$ & $\# 4 \mathrm{~b}$ & $153 \mu \mathrm{m}$ \\
\hline$\# 5 \mathrm{a}$ & $105 \mu \mathrm{m}$ & $\# 5 \mathrm{~b}$ & $206 \mu \mathrm{m}$ \\
\hline$\# 6 \mathrm{a}$ & $115 \mu \mathrm{m}$ & $\# 6 \mathrm{~b}$ & $317 \mu \mathrm{m}$ \\
\hline$\# 7 \mathrm{a}$ & $141 \mu \mathrm{m}$ & $\# 7 \mathrm{~b}$ & $328 \mu \mathrm{m}$ \\
\hline$\# 8 \mathrm{a}$ & $160 \mu \mathrm{m}$ & & \\
\hline$\# 9 \mathrm{a}$ & $179 \mu \mathrm{m}$ & & \\
\hline
\end{tabular}

Tabelle 4.4 - Bezeichnung der Schottky-Kontakte und deren Abstände zur Golddiffusionsquelle der versetzungshaltigen n-dotierten Proben.

In den Abbildungen 4.11 und 4.12 sind die DLTS-Spektren von vier Kontakten der Probe mit der höheren Versetzungsdichte $\left(3.3 \cdot 10^{8} \mathrm{~cm}^{-2}\right)$ gezeigt. Die Spektren der Kontakte \#3a und \#6a zeigen eine dominierende Linie bei ca. $260 \mathrm{~K}$, die auch bei Voss [60] in versetzungshaltigem Material gemessen wurde und aufgrund ihrer Abhängigkeit von der Goldkonzentration, ihrer Emissions- und Aufladekinetik einem Akzeptorzustand des Goldes an Versetzungen zugeordnet wurde. Diese näher zu untersuchende Linie wird im folgenden mit $\mathrm{Au}_{\mathrm{n}}$ bezeichnet.

Die Linie, die bei ca. 210K erscheint, ist die C-Linie versetzungsinduzierter Defekte in n-dotiertem Silizium. In Abb. 4.12(a) ist zu erkennen, dass sie in diesem Fall als Doppellinie $\mathrm{C}_{1}$ und $\mathrm{C}_{2}$ auftritt (Kap. 2.4.2). 

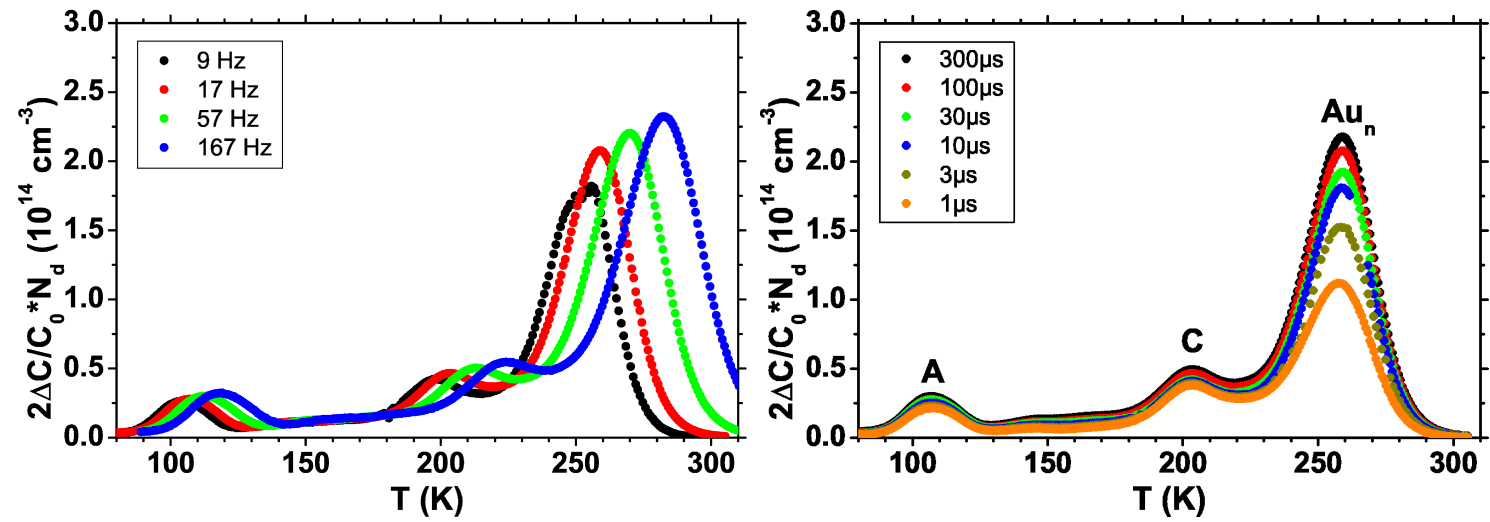

(a) $80 \mu \mathrm{m}$ Abstand zur Golddiffusionsquelle
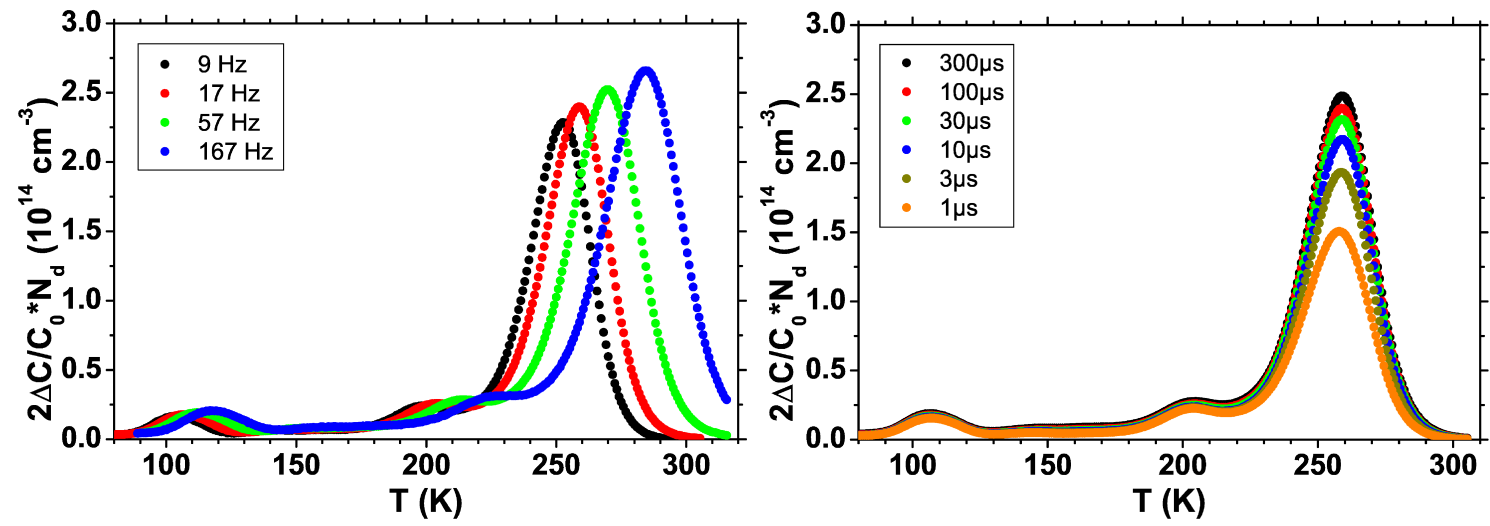

(b) $115 \mu \mathrm{m}$ Abstand zur Golddiffusionsquelle

Abbildung 4.11 - DLTS-Spektren der golddotierten Probe mit der Versetzungsdichte $3.3 \cdot 10^{8} \mathrm{~cm}^{-2}$ gemessen in der Frequenzvariation mit $t_{\mathrm{p}}=100 \mu \mathrm{s}$ und in der Pulslängenvariation mit $f_{\mathrm{w}}=17 \mathrm{~Hz}\left(U_{\mathrm{b}}=-5 \mathrm{~V}, U_{\mathrm{P}}=4.9 \mathrm{~V}\right)$. Die A- und die C-Linie treten im DLTSSpektrum versetzungshaltigen, $n$-dotierten Siliziums auf. Die Linie bei ca. $260 \mathrm{~K}$ $\left(A u_{\mathrm{n}}\right)$ ist in flacheren Bereichen, d.h. nahe der Golddiffusionsquelle, in der DLTS messbar.

Die DLTS-Linie bei ca. 110K ist eine Überlagerung mindestens zweier Linien unterschiedlicher tiefer Zustände: Zum einen wird in diesem Temperaturbereich die Linie G1 des Gold-Wasserstoff-Komplexes $\mathrm{AuH}^{(-/-)}$(siehe Kap. 2.2.3, 4.2.1) gemessen, zum anderen erscheint dort die sogenannte A-Linie des DLTS-Spektrums versetzungsbedingter Defekte in n-dotiertem Silizium ${ }^{[54]}$.

In größerer Entfernung zur Golddiffusionsquelle nimmt die Amplitude von $\mathrm{Au}_{\mathrm{n}} \mathrm{ab}$, wobei sie ab einer Tiefe von etwa $150 \mu \mathrm{m}$ nicht mehr von der C-Linie zu trennen ist (Abb. 4.12). Die Spektren der Abb4.13 wurden an der Probe mit der geringeren Versetzungsdichte $\left(1.4 \cdot 10^{8} \mathrm{~cm}^{-2}\right)$ gemessen. Auch in diesen Spektren erscheinen die oben erwähnten Linien $\left(\mathrm{Au}_{\mathrm{n}}, \mathrm{C}, \mathrm{A}\right.$ bzw. G1). Allerdings ist in dieser Probe auch bis zu ei- 

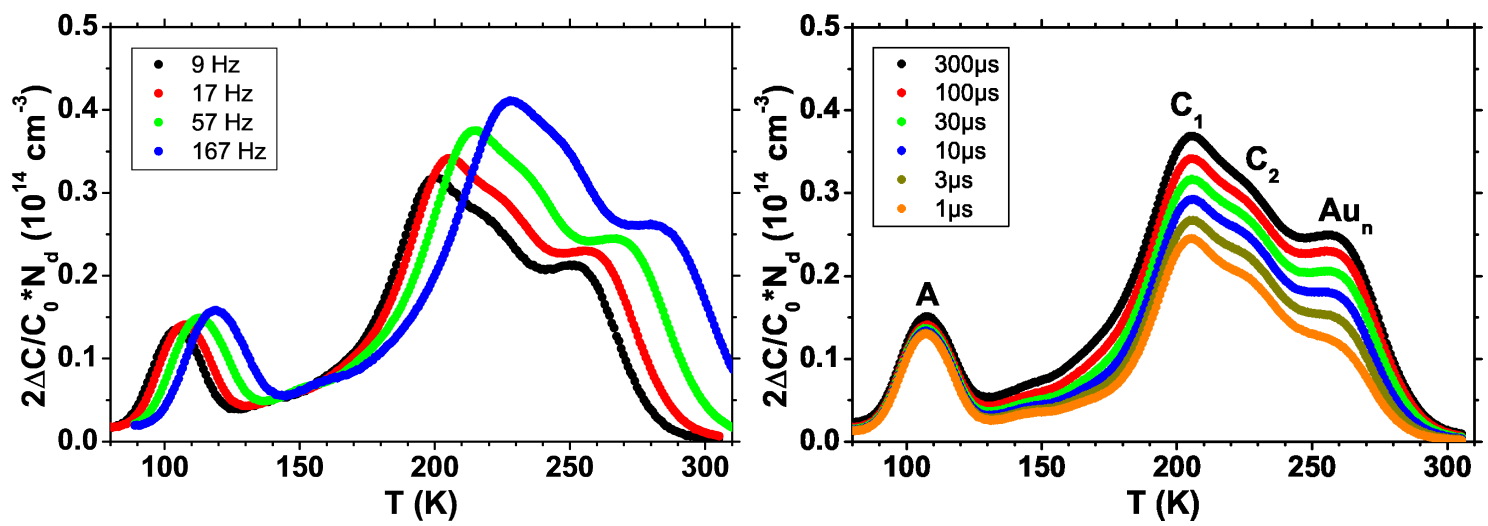

(a) $141 \mu \mathrm{m}$ Abstand zur Golddiffusionsquelle
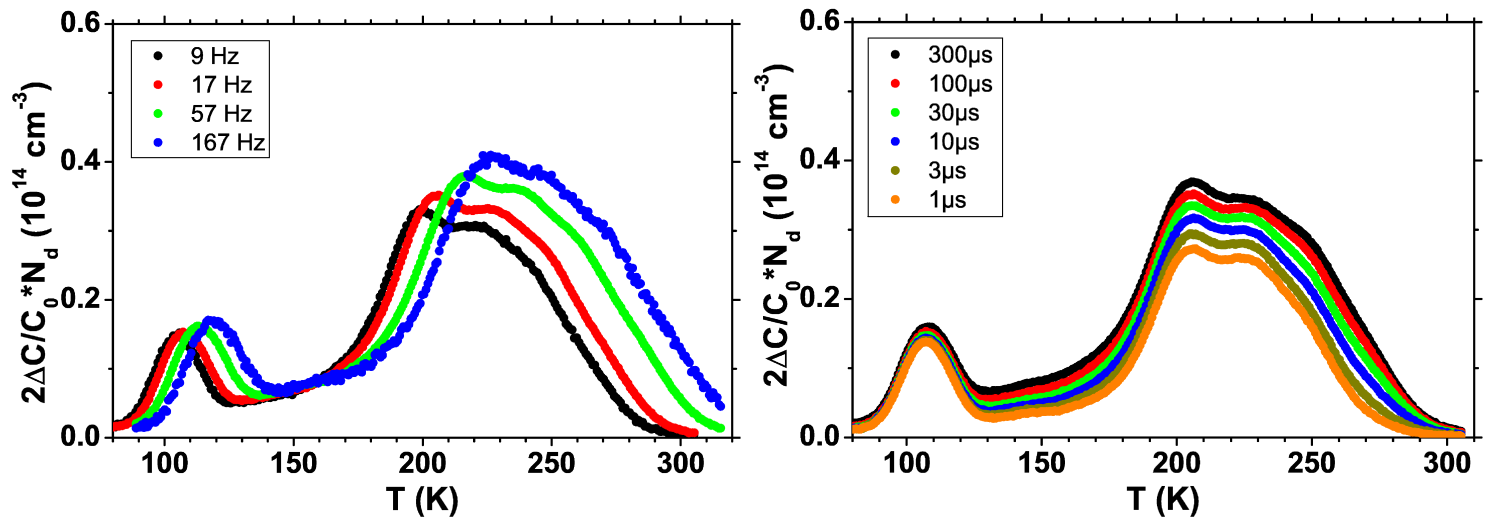

(b) $160 \mu \mathrm{m}$ Abstand zur Golddiffusionsquelle

Abbildung 4.12 - DLTS-Spektren der golddotierten Probe mit der Versetzungsdichte $3.3 \cdot 10^{8} \mathrm{~cm}^{-2}$ gemessen in der Frequenzvariation mit $t_{\mathrm{p}}=100 \mu$ s und in der Pulslängenvariation mit $f_{\mathrm{w}}=17 \mathrm{~Hz}\left(U_{\mathrm{b}}=-5 \mathrm{~V}, U_{\mathrm{P}}=4.9 \mathrm{~V}\right)$. Hier tritt die versetzungsinduzierte Linie als Doppellinie $C_{1}$ und $C_{2}$ auf. Die Linie bei ca. 260K $\left(A u_{\mathrm{n}}\right)$ ist ab einer Tiefe von ca. $150 \mu \mathrm{m}$ in der DLTS nicht mehr von den versetzungsinduzierten Defekten der C-Linien trennbar.

ner Tiefe von $330 \mu \mathrm{m}$ das Signal von $\mathrm{Au}_{\mathrm{n}}$ messbar. Dieser Zusammenhang wird in $\mathrm{Abb}$. 4.14 verdeutlicht, in der die Amplitudenwerte bei einer Einfangzeit von $t_{\mathrm{p}}=300 \mu \mathrm{s}$ (vgl. Kap.2.1.1) über dem Abstand des Kontaktes zur Golddiffusionsquelle aufgetragen ist.

Außerdem sind dort Goldkonzentrationen aufgetragen, die unter Berücksichtigung des Ladungsträgereinfangs aus dem Debye-Tail und der im Vergleich zur flachen Dotierung nicht zu vernachlässigenden Dichte tiefer Zustände berechnet wurde ${ }^{[23]}$. Diese Konzentrationen stellen nur eine untere Grenze der Konzentration dar, wenn die Einfangcharakteristik (Abb. 4.15) der betrachteten Defekte von der eines Punktdefektes abweicht. Daneben sind die Amplitudenwerte der C-Linie eingetragen. 

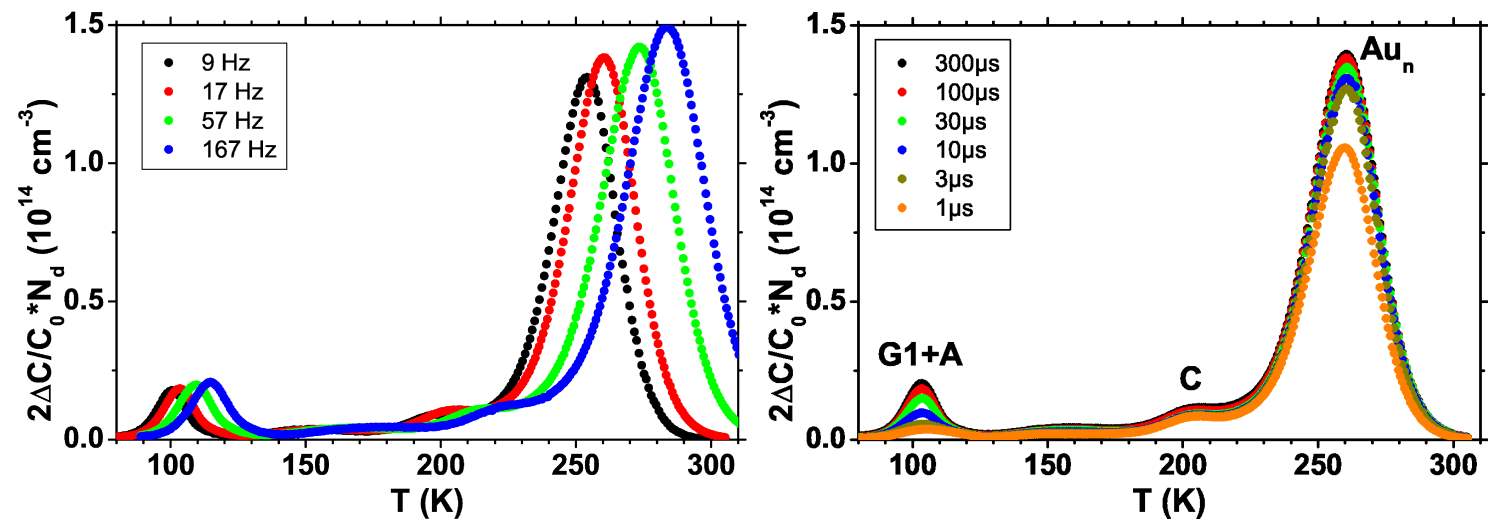

(a) $115 \mu \mathrm{m}$ Abstand zur Golddiffusionsquelle
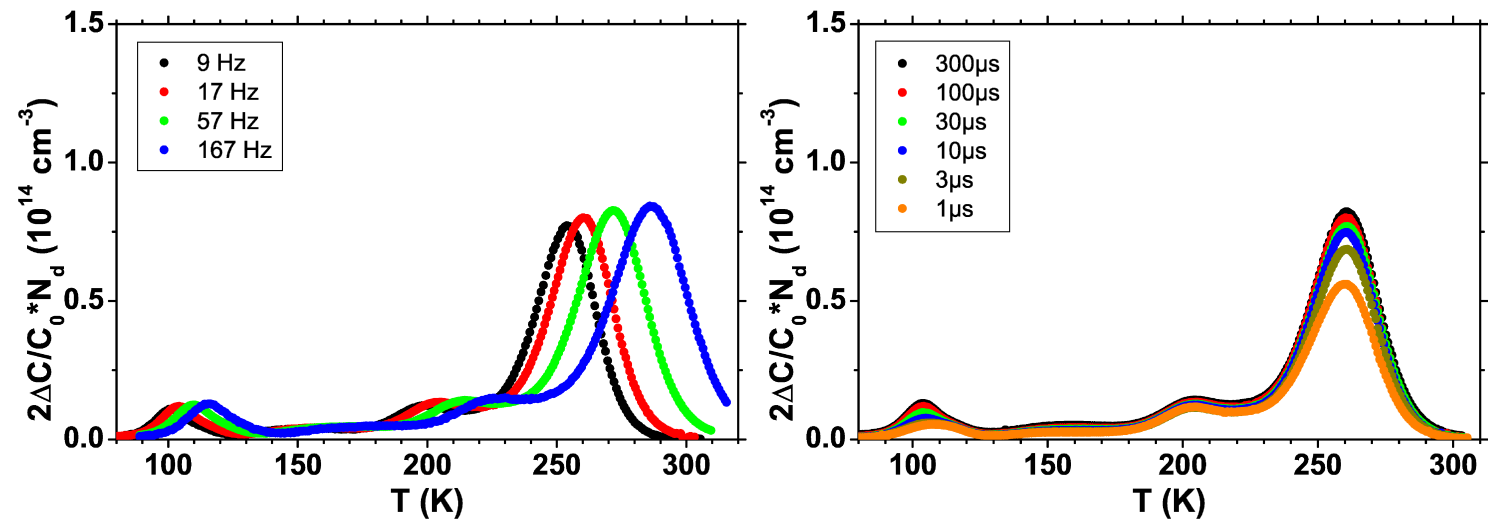

(b) $206 \mu \mathrm{m}$ Abstand zur Golddiffusionsquelle
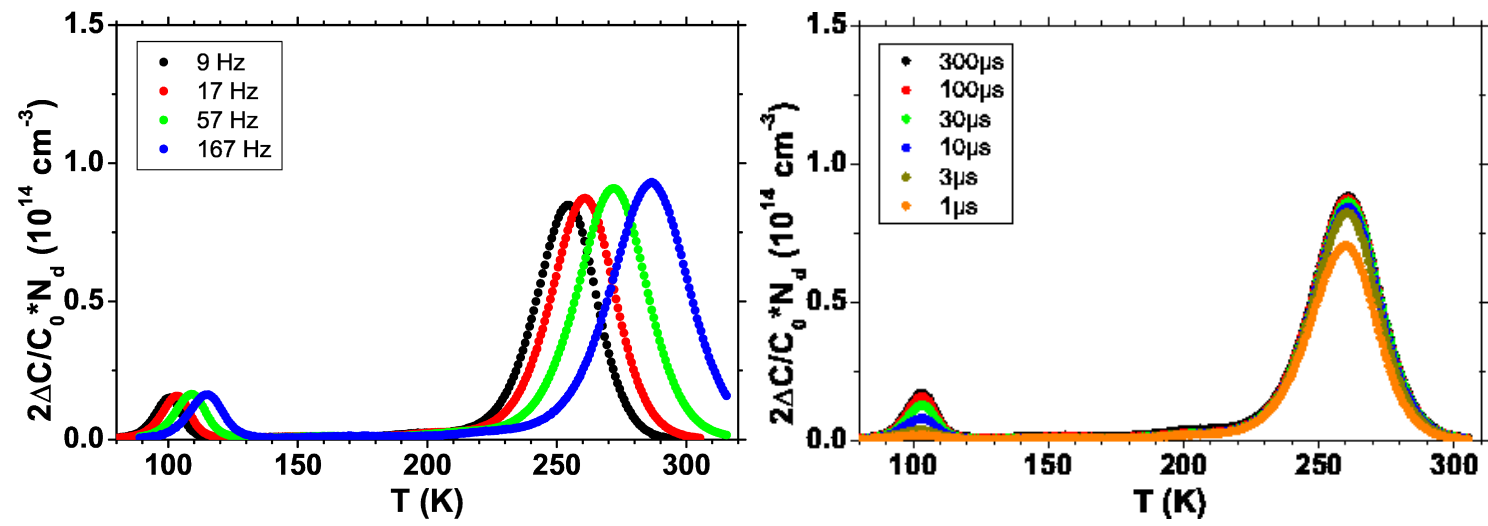

(c) $328 \mu \mathrm{m}$ Abstand zur Golddiffusionsquelle

Abbildung 4.13 - DLTS-Spektren der golddotierten Probe mit der Versetzungsdichte $1.4 \cdot 10^{8} \mathrm{~cm}^{-2}$ gemessen in der Frequenzvariation mit $t_{\mathrm{p}}=100 \mu \mathrm{s}$ und in der Pulslängenvariation mit $f_{\mathrm{w}}=17 \mathrm{~Hz}\left(U_{\mathrm{b}}=-5 \mathrm{~V}, U_{\mathrm{P}}=4.9 \mathrm{~V}\right)$. Die C-Linie tritt im DLTS-Spektrum versetzungshaltigen, $n$-dotierten Siliziums ebenso auf, wie die A-Linie. Diese wird z.B. in der Messung am Kontakt \#3b $(115 \mu \mathrm{m})$ von der Linie des Gold-WasserstoffKomplexes (G1, vgl. Kap.4.2.1.1) überlagert. Die Linie bei ca. $260 \mathrm{~K}\left(A u_{\mathrm{n}}\right)$ ist in der Probe mit der geringeren Versetzungsdichte $\left(1.4 \cdot 10^{8} \mathrm{~cm}^{-2}\right)$ in allen Abständen zur Golddiffusionsquelle messbar. 


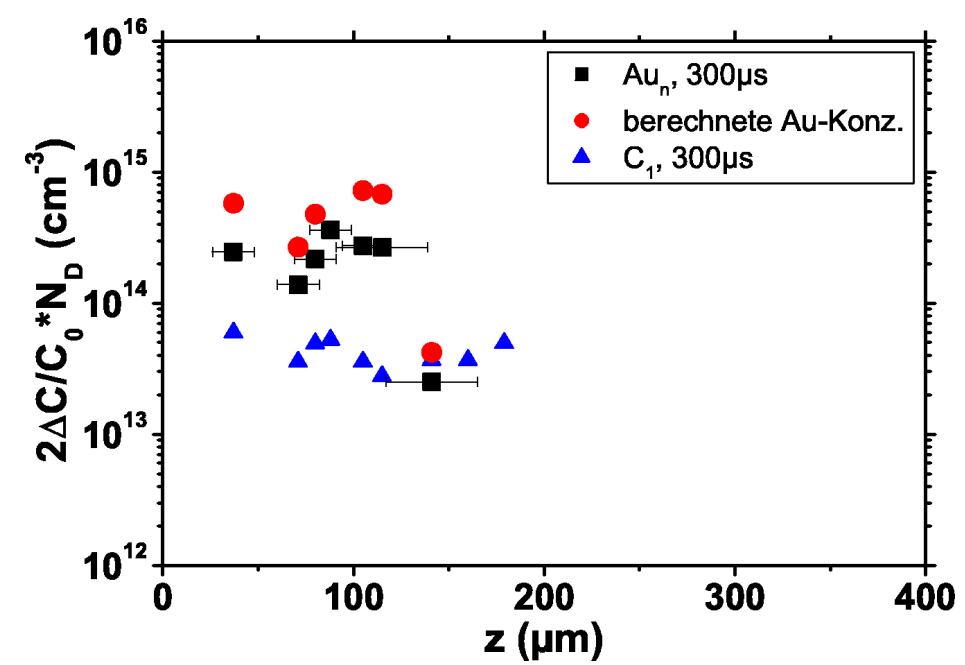

(a) Versetzungsdichte $3.3 \cdot 10^{8} \mathrm{~cm}^{-2}$

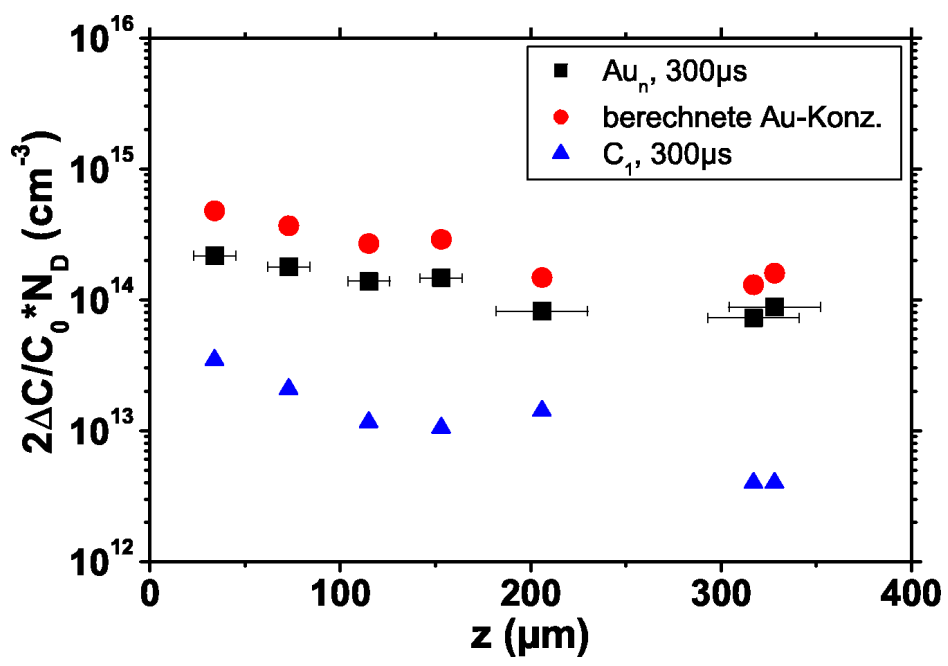

(b) Versetzungsdichte $1.4 \cdot 10^{7} \mathrm{~cm}^{-2}$

Abbildung 4.14 - Amplitudenwerte der $A u_{n}$-Linie gemessen bei $t_{\mathrm{p}}=300 \mu$ s über dem Abstand des entsprechenden Kontaktes zur Golddiffusionsquelle. Ab einer Tiefe von ca. $150 \mu \mathrm{m}$ ist die Linie $A u_{\mathrm{n}}$ in der Probe mit der höheren Versetzungsdichte nicht vom Signal der C-Linie zu trennen. In der Probe mit der geringeren Versetzungsdichte ist $A u_{\mathrm{n}}$ auch in einer Tiefe von ca. $330 \mu \mathrm{m}$ noch messbar. Die Kreise zeigen die aus den Werten der Amplituden nach dem Modell von Pons $\underline{\text { [23] }}$ berechneten Konzentrationen. Die Fehlerbalken zeigen den Bereich, über den aufgrund der Größe des Kontaktes gemittelt wird. 


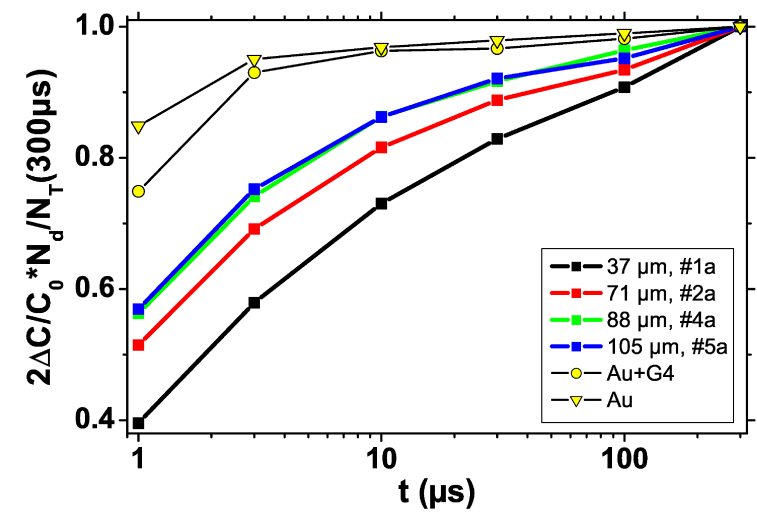

(a) Versetzungsdichte $3.3 \cdot 10^{8} \mathrm{~cm}^{-2}$

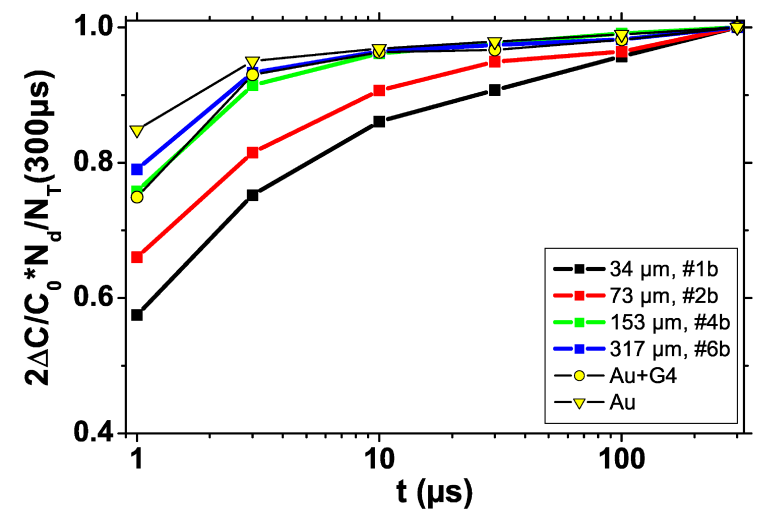

(b) Versetzungsdichte $1.4 \cdot 10^{8} \mathrm{~cm}^{-2}$

Abbildung 4.15 - Vergleich der Einfangkinetiken der Linie $A u_{n}$ mit denen der Linien $A u+G 4$ bzw. $A u$ in versetzungsfreiem Material. Aufgetragen ist der Wert der Amplitude normiert auf den Wert gemessen bei einer Einfangzeit von $t_{\mathrm{p}}=300 \mu \mathrm{s}$ über der Einfangzeit. In versetzungsfreiem Silizium können die Defekte schon ab einer Einfangzeit von ca. $10 \mu$ s nahezu komplett umgeladen werden. In versetzungshaltigem Material sind nur die Einfangkinetiken der Probe mit der geringeren Versetzungsdichte und für die größten Abstände zur Golddiffusionsquelle mit denen in versetzungsfreiem Material vergleichbar.

Die DLTS-Linie $\mathrm{Au}_{\mathrm{n}}$, deren Amplitude abhängig von der Versetzungsdichte mit zunehmendem Abstand zur Golddiffusionsquelle abnimmt, soll im folgenden näher untersucht werden.

In der Tabelle 4.5 sind die durchschnittlichen Breiten der Linien $\mathrm{Au}+\mathrm{G} 4$ und $\mathrm{Au}$ mit denen der Linie $\mathrm{Au}_{\mathrm{n}}$ verglichen. $\mathrm{Au}_{\mathrm{n}}$ erscheint demnach unverbreitert im Vergleich zu den goldinduzierten Linien im versetzungsfreiem Silizium. Zur Bestimmung der Emissions-

\begin{tabular}{|l|l|}
\hline $\mathrm{Au}+\mathrm{G} 4$ & $30.9 \mathrm{~K}$ \\
\hline $\mathrm{Au}$ & $28.7 \mathrm{~K}$ \\
\hline $\mathrm{Au}{ }_{\mathrm{n}}$, höhere Versetzungsdichte & $29.8 \mathrm{~K}$ \\
\hline $\mathrm{Au}_{\mathrm{n}}$, geringe Versetzungsdichte & $29.3 \mathrm{~K}$ \\
\hline
\end{tabular}

Tabelle 4.5 - Durchschnittliche Linienbreiten der im versetzungshaltigen Material gemessenen, goldkonzentrationsabhängigen DLTS-Linie und der Linie, die in versetzungsfreiem Material gemessen wurde.

eigenschaften sind in Abb. 4.16 die Arrheniusauftragungen der thermischen Emissionsraten dargestellt. Es fällt auf, dass die Steigungen der zu den $A u_{n}$ gehörenden Kurven im Rahmen der experimentellen Genauigkeit mit denen der Linien $\mathrm{Au}+\mathrm{G} 4 \mathrm{bzw}$. $\mathrm{Au}$ gut übereinstimmen. Die durch lineare Regression ermittelte, durchschnittliche Aktivierungsenthalpie des zu Au $u_{n}$ gehörenden Defekts beträgt 0.536(17)eV. Dagegen erscheinen insbesondere die Kurven der $\mathrm{Au}_{\mathrm{n}}$ im stärker versetzungshaltigem Material zu tieferen 
Temperaturen verschoben im Vergleich zu der Linie im Material mit weniger Versetzungen. Bei der Bewertung dieses Umstandes muss berücksichtigt werden, dass aufgrund der Überlagerung von $\mathrm{Au}_{\mathrm{n}}$ mit der C-Linie eine solche Verschiebung des Linienmaximums von $\mathrm{Au}_{\mathrm{n}} \mathrm{zu}$ erwarten ist, und zwar umso stärker, je größer die Amplitude der C-Linie

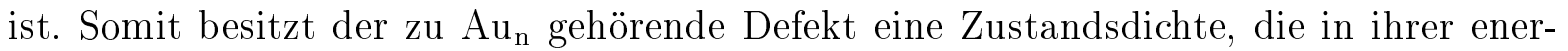
getischen Lage und ihrer Breite vergleichbar ist mit der von Gold in versetzungsfreiem Silizium, $\mathrm{Au}^{(0 /-)}$ bzw. $\mathrm{Au}^{(0 /-)}+\mathrm{AuH}^{(0 /-)}$.

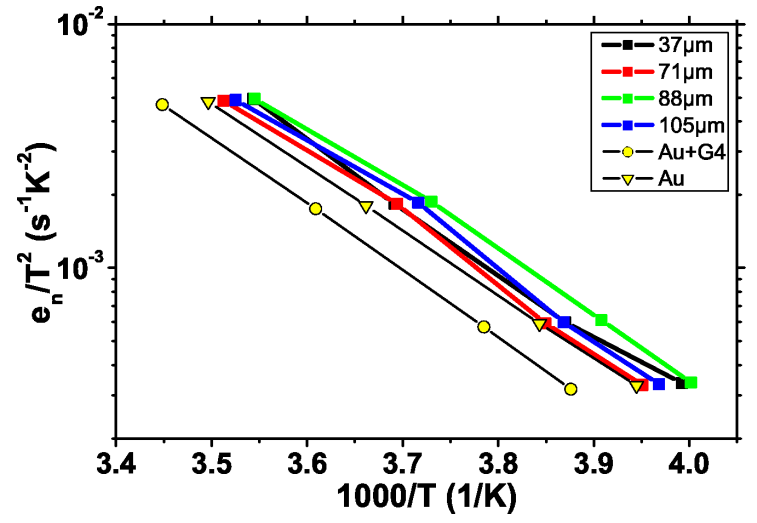

(a) Versetzungsdichte $3.3 \cdot 10^{8} \mathrm{~cm}^{-2}$

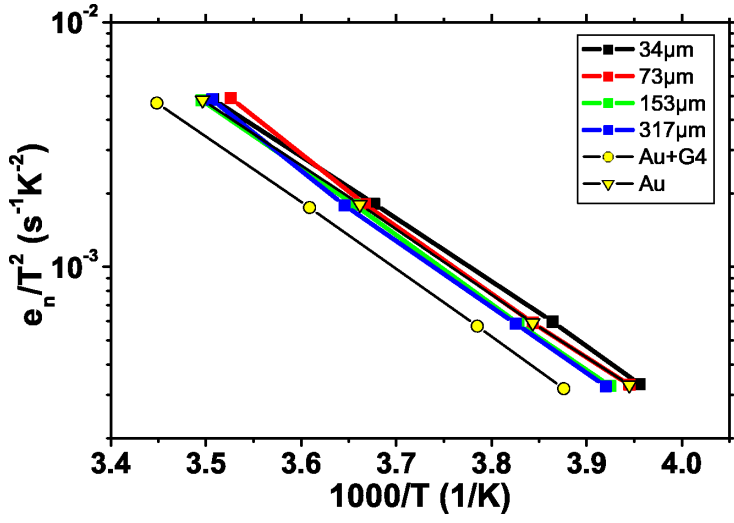

(b) Versetzungsdichte $1.4 \cdot 10^{7} \mathrm{~cm}^{-2}$

Abbildung 4.16 - Vergleich der Emissionscharakteristiken der Linie $A u_{\mathrm{n}}$ mit denen der Linien $A u+G 4$ bzw. $A u$ in versetzungsfreiem Material. In der Probe mit der größeren Versetzungsdichte liegen die Verläufe im Vergleich zu dem der Linie Au zu tieferen Temperaturen verschoben. In der Probe mit der geringeren Versetzungsdichte liegen die Verläufe in guter Übereinstimmung mit dem Verlauf der Linie Au.

Der Vergleich der $t_{\mathrm{p}}$-Variationsmessungen in der Abbildung 4.4 mit der in mit Abbildung 4.11 zeigt einen Unterschied in der Einfangcharakteristik der entsprechenden Defekte. Diese an den unterschiedlichen Kontakten gemessenen Einfangkinetiken sind in Abb. 4.15 zusammen mit denen von $\mathrm{Au}^{(0 /-)}$ bzw. $\mathrm{Au}^{(0 /-)}+\mathrm{AuH}^{(0 /-)}$ dargestellt. Dazu wurde die auf den maximalen Wert, der bei einer Einfangzeit von $t_{\mathrm{p}}=300 \mu$ s gemessen wurde, normierte Amplitude über der Einfangzeit aufgetragen.

Es fällt auf, dass es in der Probe mit der höheren Versetzungsdichte keinen Kontakt gab, an dem die Einfangcharakteristik der Linie $\mathrm{Au}_{\mathrm{n}}$ mit der in versetzungsfreiem Silizium vergleichbar ist. Nur die Messungen an den beiden Kontakte mit der größten Entfernung zur Golddiffusionsquelle $(\# 6 \mathrm{~b}, \# 7 \mathrm{~b})$ in der Probe mit der kleineren Versetzungsdichte zeigen ein vergleichbares Verhalten. Von den Spektren der Probe mit der kleineren Versetzungsdichte wurde daher eine numerische Anpassung mit den $\mathrm{Pa}-$ rametern vorgenommen, die aus den versetzungsfreien Proben ermittelt wurden. Dabei wurden nur die Dichten der Punktdefekte als Anpassungsparameter zugelassen. In Abb. 4.17 sind die Ergebnisse dieser Anpassung dargestellt. 

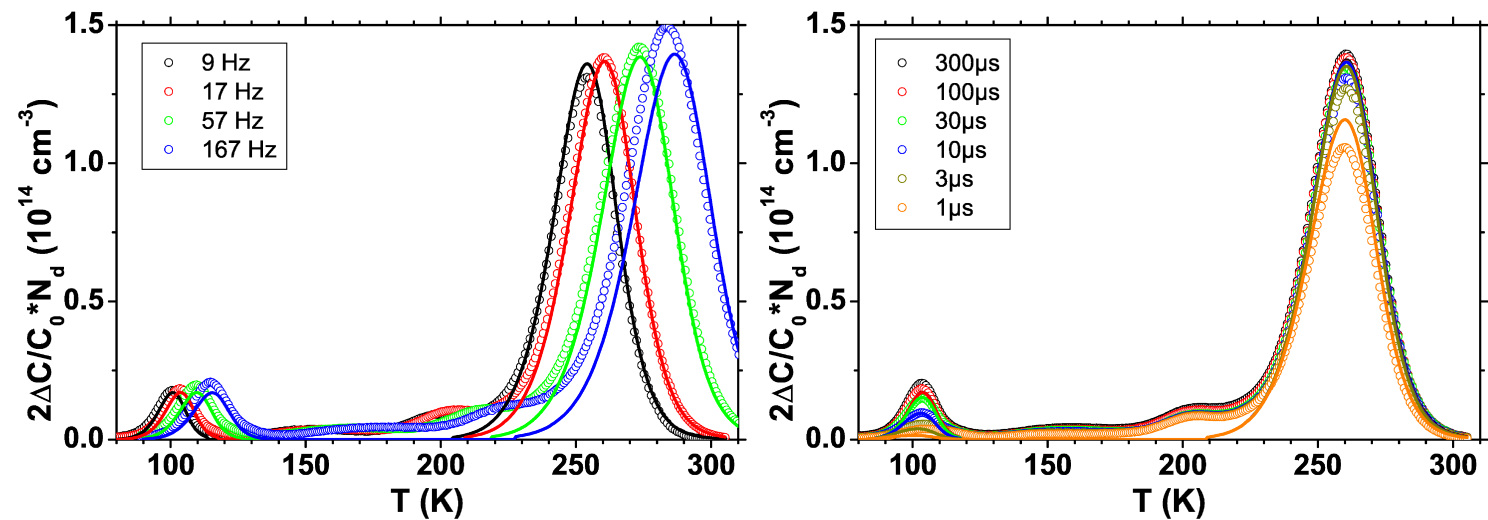

(a) $115 \mu \mathrm{m}$ Abstand zur Golddiffusionsquelle
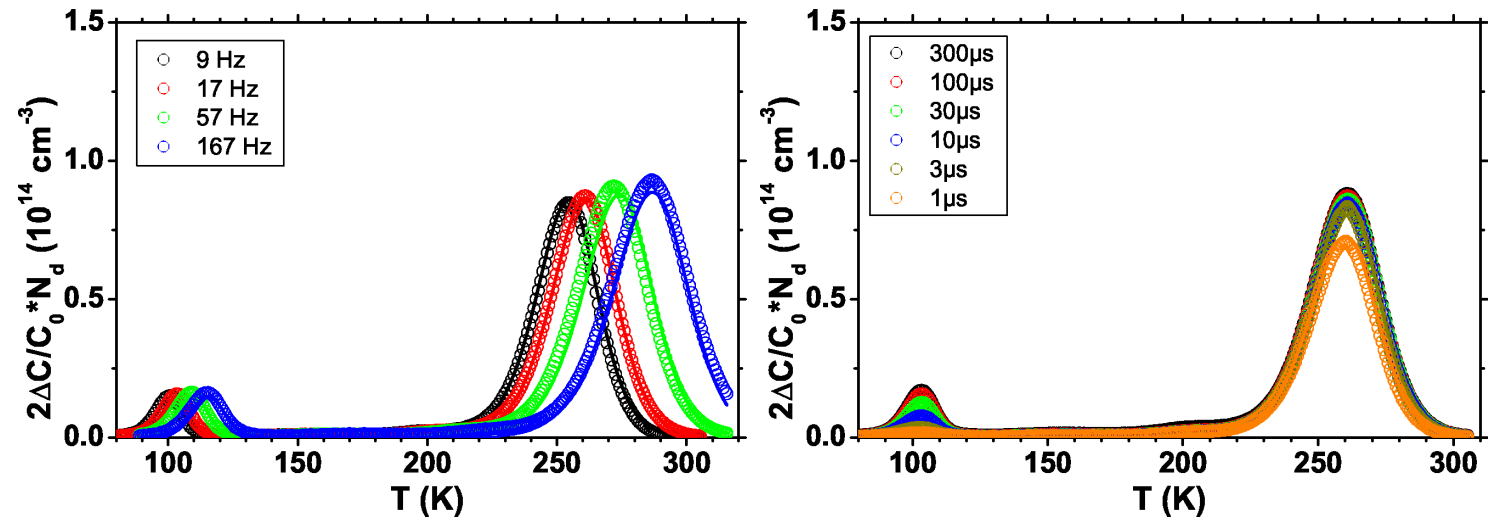

(b) $328 \mu \mathrm{m}$ Abstand zur Golddiffusionsquelle

Abbildung 4.17 - DLTS-Spektren der golddotierten Probe mit der Versetzungsdichte $1.4 \cdot 10^{8} \mathrm{~cm}^{-2}$ gemessen in der Frequenzvariation mit $t_{\mathrm{p}}=100 \mu \mathrm{s}$ und in der Pulslängenvariation mit $f_{\mathrm{w}}=17 \mathrm{~Hz}\left(U_{\mathrm{b}}=-5 \mathrm{~V}, U_{\mathrm{P}}=4.9 \mathrm{~V}\right)$. Die durchgezogenen Linien stellen das Ergebnis der numerischen Anpassung mit den Parametern der versetzungsfreien Proben dar, wobei nur die Dichten der Defekte als Anpassungsparameter variiert wurden. Deren Werte sind in Tab.4.6 aufgelistet. Zu erkennen ist, dass die numerische Anpassung nur für das Spektrum am Kontakt mit dem Abstand von $328 \mu \mathrm{m}$ zur Golddiffusionsquelle zu einer guten Übereinstimmung mit dem Messergebnis führt.

In der Tab. 4.6 sind die Ergebnisse der Anpassung eingetragen.

Aus den Abbildungen 4.16, 4.15(b) und 4.17(a) wird zum einen ersichtlich, dass die Emissionscharakteristik von $\mathrm{Au}_{\mathrm{n}}$ gut mit der des $\mathrm{Au}^{(0 /-)}$ übereinstimmt. Zum anderen stimmen jedoch die Einfangkinetiken dieser Defekte nicht überein. QUEISSER [27] hat den Einfluss der Einfangbarriere eines Vielelektronendefektes mit einem Energieniveau auf die Emissionscharakteristik untersucht und kommt zu dem Ergebnis, dass eine Einfangbarriere keinen signifikanten Einfluss auf die Form der zugehörigen Linie im DLTS-Spektrum und auf den Verlauf der Kapazitätstransienten hat. Offensichtlich 


\begin{tabular}{|c|c|c|}
\hline & \multicolumn{2}{|c|}{ Dichte $\left(10^{14} \mathrm{~cm}^{-3}\right)$} \\
\hline Defekt & $115 \mu \mathrm{m}$ & $328 \mu \mathrm{m}$ \\
\hline $\mathrm{AuH} \mathrm{H}^{(-/--)}$ & 0.21 & 0.197 \\
\hline $\mathrm{AuH} \mathrm{H}^{(0 /-)}$ & 2.05 & 1.25 \\
\hline $\mathrm{Au}{ }^{(0 /-)}$ & 0.21 & 0.197 \\
\hline
\end{tabular}

Tabelle 4.6 - Aus den numerischen Anpassungen ermittelte Defektdichten des Kontaktes

handelt es sich bei dem zu der Linie $A u_{n}$ gehörenden Defekt um einen Punktdefekt mit Einfangbarriere.

Eine weitere Auffälligkeit sollte kurz erwähnt werden: In der Probe mit der größeren Versetzungsdichte ist die Einfangkinetik korreliert mit dem Abstand zur Golddiffusionsquelle. Der Verlauf der Einfangkinetik wird flacher, d.h. die Besetzung bei gleicher Einfangzeit wird höher, je weiter der zugehörige Kontakt von der Golddiffusionsquelle entfernt lag. Hier ist jedoch keine Korrelation mit der C-Linienamplitude festzustellen (vgl. Abb. 4.15(a) und 4.14(a)). Anders verhält es sich bei der Probe mit der geringeren Versetzungsdichte: Hier gibt es keine Korrelation der Einfangkinetik mit der Entfernung zur Golddiffusionsquelle. Jedoch ist hier der Verlauf der Einfangkinetik flacher, je kleiner die Amplitude der entsprechenden C-Linie ist (vgl. Abb. 4.15(b) und 4.14(b)).

Für den Fall, dass es aufgrund hoher Dichten tiefer Zustände zu kompensatorischen Effekten kommt, kann die temperaturabhängige Messung der Dichte freier Ladungsträger Aufschluss über die Dichte der Zustände geben. Daher wurde an jedem Kontakt, an dem DLTS-Spektren gemessen wurden, obligatorisch vor der ersten DLTS-Messung in Abständen von 10K die Kapazitäts-Spannungskennlinie gemessen. Aus dieser lässt sich bei Kenntnis des Kontaktdurchmessers die Dichte freier Ladungsträger bestimmen ${ }^{[22]}$. Der Kontaktdurchmesser wurde durch Fotografieren der Kontakte und anschließendem Ausmessen der vergrößerten Aufnahmen mit einem relativen Fehler von 5\% bestimmt. Da der Kontaktdurchmesser mit der vierten Potenz in die Berechnung der Dichte freier Ladungsträger eingeht, liegt der resultierende relative Fehler dieser Dichte bei etwa 20\%. Die Zeitkonstante der Kapazitätsmessung, d.h. die Dauer für das Durchlaufen des gesamten Spannungsbereich mit konstanter Spannungsänderung über die Zeit, beträgt 23s. Wenn die Emissionsrate der tiefen Zustände in einem bestimmten Temperaturbereich vergleichbar mit dieser Zeitkonstante ist und eine ausreichend hohe Dichte dieser Zustände zu kompensatorischen Effekten führt, sollte in dem besagten Temperaturbereich eine Änderung der Dichte freier Ladungsträger zu verzeichnen sein. Die Abb. 4.18 zeigt den Verlauf der temperaturabhängigen Dichte freier Ladungsträger der beiden n-dotierten Proben für die Kontakte mit den angezeigten Abständen zur Golddiffusionsquelle. Als Vergleich ist jeweils der Verlauf der golddotierten versetzungsfreien Probe ebenfalls dargestellt. Es fällt auf, dass in beiden Proben im Temperaturbereich um $210 \mathrm{~K}$ einer Änderung der freien Ladungsträgerdichte zwischen 1.5 und $3.5 \cdot 10^{14} \mathrm{~cm}^{-3}$ 
gemessen wird. Für die Probe mit der höheren Versetzungsdichte gilt dies bis zum Abstand von $115 \mu \mathrm{m}$ zur Golddiffusionsquelle. Dies ist der Abstand, bis zu dem die Linie $\mathrm{Au}_{\mathrm{n}}$ separat gemessen werden kann. Darüber hinaus scheint die Ladungsträgerdichte für Kontakte in der Nähe der Golddiffusionsquelle in der Probe mit der höheren Versetzungsdichte über den gesamten Temperaturbereich um rund $6 \cdot 10^{14} \mathrm{~cm}^{-3}$ zu geringeren Ladungsträgerdichten verschoben (Abb. 4.18(a)). Für die Kontakte jenseits der $115 \mu \mathrm{m}$ ist hier nur ein leichter Anstieg über einen breiten Temperaturbereich zu verzeichnen. Bei der Probe mit der geringeren Versetzungsdichte ist die Diskrepanz zwischen den Ladungsträgerdichten im oberen Temperaturbereich kleiner (Abb. 4.18(b)) . Hier liegt die gemessene Ladungsträgerdichte für Kontakte nahe der Golddiffusionsquelle bei 8 bis $9 \cdot 10^{14} \mathrm{~cm}^{-3}$. Die versetzungsfreie Probe, die aus dem gleichen Ausgangsmaterial mit den Nenndotierung von $10^{15} \mathrm{~cm}^{-3}$ hergestellt wurde, zeigt keine auffälligen Veränderungen in der Ladungsträgerdichte über den gesamten Temperaturbereich. 


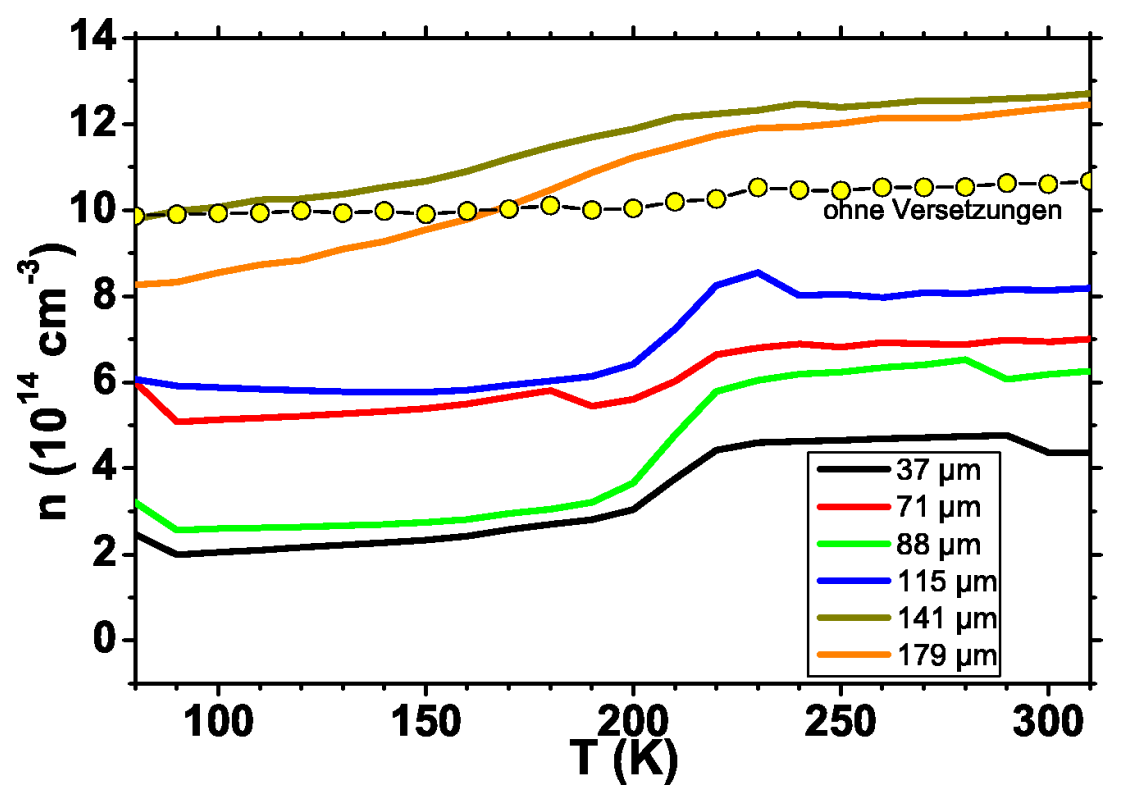

(a) Versetzungsdichte $3.3 \cdot 10^{8} \mathrm{~cm}^{-2}$

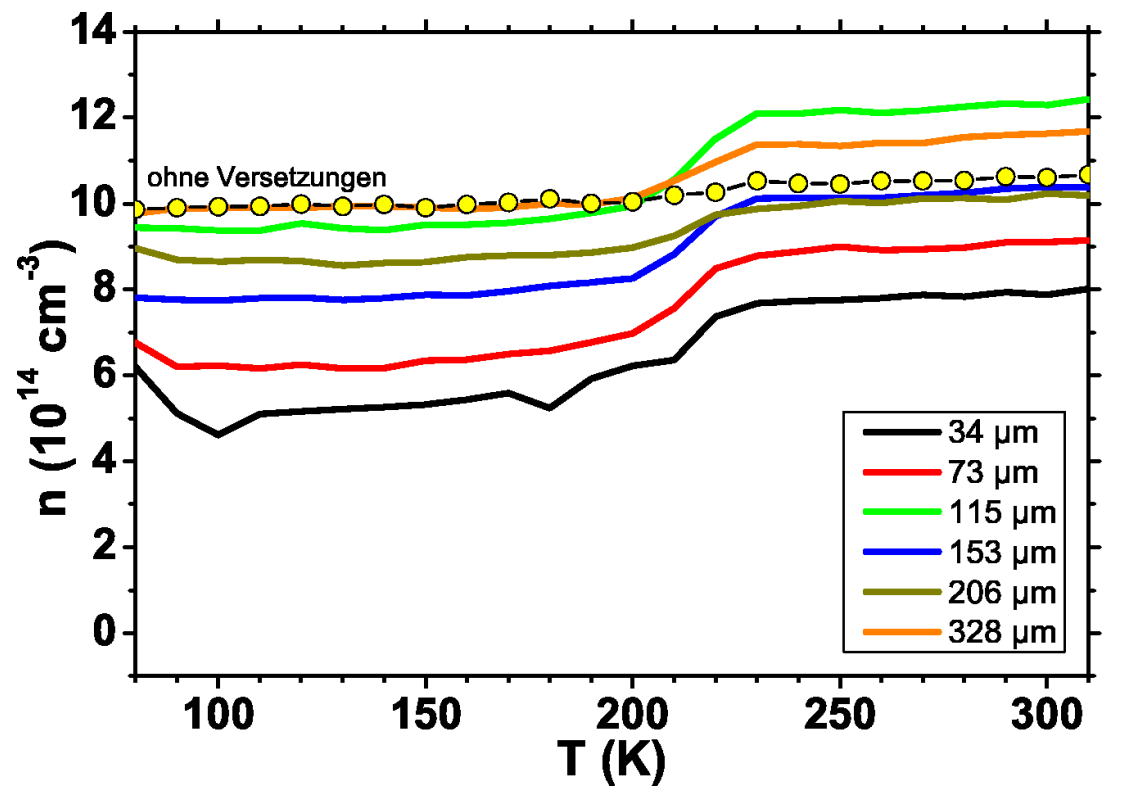

(b) Versetzungsdichte $1.4 \cdot 10^{8} \mathrm{~cm}^{-2}$

Abbildung 4.18 - Dichte freier Ladungsträger in Abhängigkeit von der Temperatur für die Probe mit der Versetzungsdichte $3.3 \cdot 10^{8} \mathrm{~cm}^{-2}$ (a) und mit der Versetzungsdichte $1.4 \cdot 10^{8} \mathrm{~cm}^{-2}$ (b). Als Vergleich ist der entsprechende Verlauf für die golddotierte versetzungsfreie Probe dargestellt. In der Probe mit der größeren Versetzungsdichte gibt es für einige Kontakte eine deutliche Verschiebung zu geringeren Dichten freier Elektronen. Dieser Effekt fällt in der Probe mit der geringeren Versetzungsdichte nicht so deutlich auf. 


\section{Zusammenfassung}

In der folgenden Aufzählung sollen alle bisher untersuchten Eigenschaften von $\mathrm{Au}_{n}$ bzw. des zugehörigen Defektes zusammengefasst werden:

1. Die Amplitude der DLTS-Linie $\mathrm{Au}_{n}$ verringert sich mit zunehmendem Abstand zur Golddiffusionsquelle, wobei eine höhere Versetzungsdichte diesen Effekt verstärkt.

2. Die Position im DLTS-Spektrum sowie die Linienbreite stimmt mit der von Gold in versetzungsfreiem Silizium überein.

3. Die durch die Arrheniusauftragung ermittelte Aktivierungsenthalpie des Defektes liegt zwischen der vom Goldakzeptorzustand und dem Akzeptorzustand des GoldWasserstoff-Komplexes in versetzungsfreiem Silizium.

4. Die Temperatur des Linienmaximums erscheint zu tieferen Temperaturen verschoben und zwar umso mehr, je höher die Amplitude der versetzungsinduzierten CLinie ist.

5. Die Einfangcharakteristik ist nur in der Probe mit der geringeren Versetzungsdichte und an den Kontakten mit der größten Entfernung zur Golddiffusionsquelle wie bei einem Punktdefekt und vergleichbar mit der Einfangcharakteristik der Akzeptorzustände der goldinduzierten Defekte in versetzungsfreiem Silizium.

6. Die Dichte freier Ladungsträger zeigt in den unterschiedlichen Proben gleiche Abhängigkeiten im Temperaturbereich um 210K. Dort, wo die Linie $\mathrm{Au}_{\mathrm{n}}$ nicht mehr separat gemessen werden kann, ist diese Abhängigkeit nicht zu verzeichnen.

7. In der Probe mit der hohen Versetzungsdichte ist die Ladungsträgerdichte für Kontakte in der Nähe der Golddiffusionsquelle über den gesamten Temperaturbereich signifikant zu tieferen Dichten verschoben. 


\subsubsection{2 p-dotiertes Silizium}

Die Erzeugung von Versetzungen in der p-dotierten Probe erfolgte wie die der n-dotierten Probe mit der höheren Versetzungsdichte. Auch hier konnte durch die schon beschriebene Schrägschlifftechnik (siehe Kap. 3.5) der Einfluss der Goldkonzentration auf das Spektrum versetzungshaltiger p-dotierter Siliziumproben gemessen werden. Die Aluminiumkontakte wurden wie bei der p-dotierten versetzungsfreien Probe präpariert, die nach dem HF-Dip dem Licht einer Halogenlampe ausgesetzt wurde. In Tabelle 4.7 sind die Bezeichnungen der gemessenen Kontakte beschrieben. In der Abb. 4.19 sind DLTS-

\begin{tabular}{|l|l|}
\hline$\# 1 \mathrm{c}$ & $23 \mu \mathrm{m}$ \\
\hline$\# 2 \mathrm{c}$ & $31 \mu \mathrm{m}$ \\
\hline$\# 3 \mathrm{c}$ & $43 \mu \mathrm{m}$ \\
\hline$\# 4 \mathrm{c}$ & $50 \mu \mathrm{m}$ \\
\hline$\# 5 \mathrm{c}$ & $61 \mu \mathrm{m}$ \\
\hline$\# 6 \mathrm{c}$ & $65 \mu \mathrm{m}$ \\
\hline$\# 7 \mathrm{c}$ & $81 \mu \mathrm{m}$ \\
\hline$\# 8 \mathrm{c}$ & $102 \mu \mathrm{m}$ \\
\hline$\# 9 \mathrm{c}$ & $136 \mu \mathrm{m}$ \\
\hline$\# 10 \mathrm{c}$ & $140 \mu \mathrm{m}$ \\
\hline$\# 11 \mathrm{c}$ & $157 \mu \mathrm{m}$ \\
\hline$\# 12 \mathrm{c}$ & $174 \mu \mathrm{m}$ \\
\hline$\# 13 \mathrm{c}$ & $177 \mu \mathrm{m}$ \\
\hline$\# 14 \mathrm{c}$ & $195 \mu \mathrm{m}$ \\
\hline
\end{tabular}

Tabelle 4.7 - Bezeichnung der Schottky-Kontakte und deren Abstände zur Golddiffusionsquelle in der p-dotierten Probe.

Spektren der Kontakte \#2c, \#3c, \#8c und \#10c, die mit einer Sperrspannung von 5V und einer Pulsspannung von 4.1V gemessen wurden, zusammen mit dem Ergebnis einer numerischen Anpassung dargestellt.

Alle in der Abb. 4.19 dargestellten Spektren zeigen eine dominierende Linie bei 155K. Diese Linie konnte in den Spektren der Kontakte \#2c und \#3c mit den gleichen Parametern angepasst werden, wie die Linie des Golddonators in versetzungsfreiem Silizium. Diese Linie wird im folgenden mit $A u_{p}$ bezeichnet. Die Linie bei $262 \mathrm{~K}$ konnte ebenfalls durch die bei der Analyse der p-dotierten versetzungsfreien Probe gewonnenen Parametern für die $\mathrm{Au}+\mathrm{G} 4$-Linie angepasst werden. Obwohl gemäß der Kontaktpräparation, bei der die Probe vor dem Aufdampfen der Aluminiumkontakte dem Licht einer Halogenlampe ausgesetzt war, die Linie des Gold-Wasserstoff-Komplexes G2 $\left(\mathrm{AuH}^{(+/ 0)}\right) \mathrm{zu}$ erwarten wäre, tritt diese in der versetzungshaltigen Probe kaum in Erscheinung. 

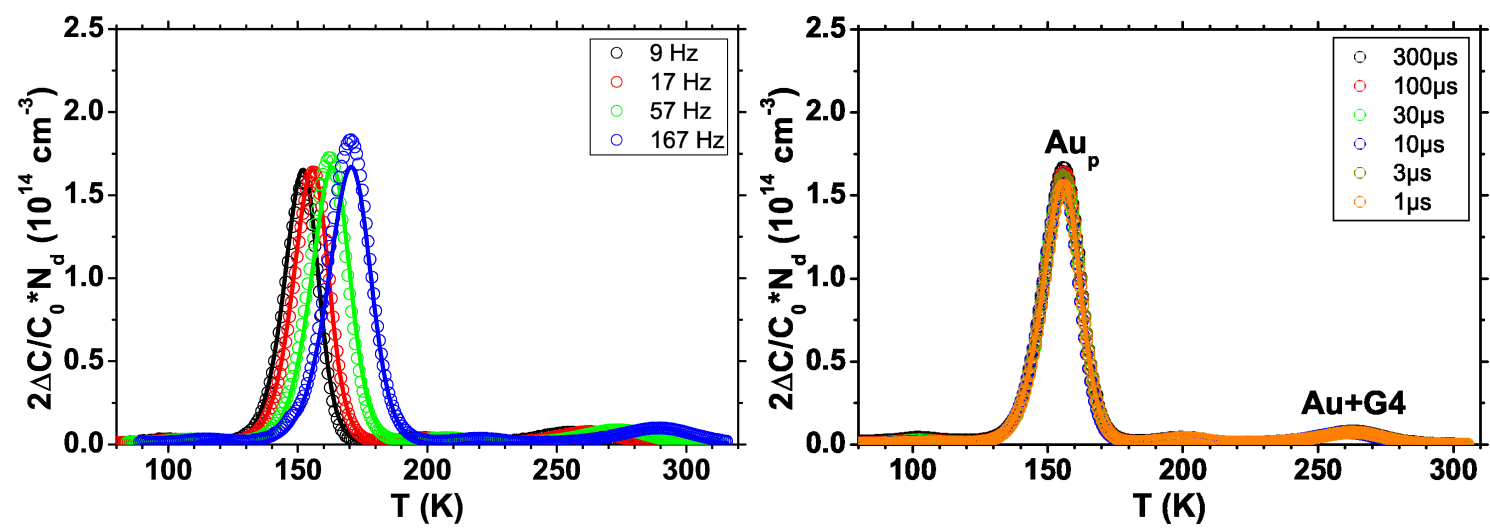

(a) $31 \mu \mathrm{m}$ Abstand zur Golddiffusionsquelle
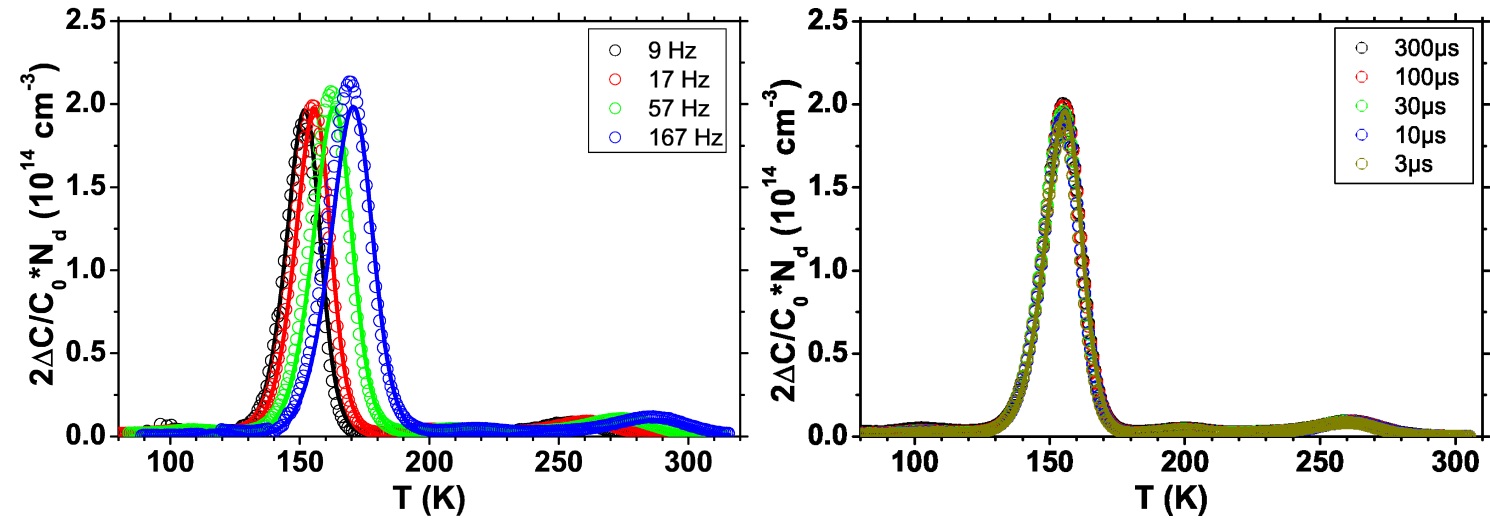

(b) $50 \mu \mathrm{m}$ Abstand zur Golddiffusionsquelle
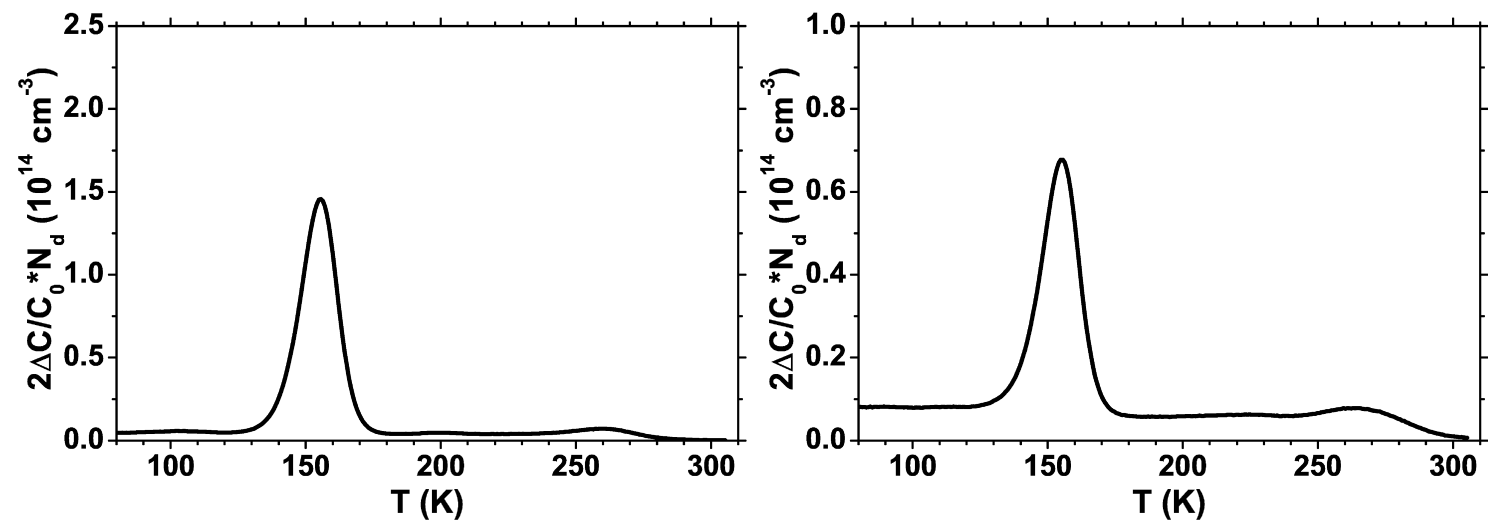

(c) $102 \mu \mathrm{m}$ (links) und $140 \mu \mathrm{m}$ (rechts) zur Golddiffusionsquelle

Abbildung 4.19 - DLTS-Spektren der golddotierten Probe mit der Versetzungsdichte $3.3 \cdot 10^{8} \mathrm{~cm}^{-2}$, a) gemessen in der Frequenzvariation mit $t_{\mathrm{p}}=100 \mu \mathrm{s}$ und in der Pulslängenvariation mit $\left.\left.f_{\mathrm{w}}=17 \mathrm{~Hz}\left(U_{\mathrm{b}}=-5 \mathrm{~V}, U_{\mathrm{P}}=4.1 \mathrm{~V}\right), b\right), c\right)$ gemessen bei $t_{\mathrm{p}}=300 \mu \mathrm{s}, f_{\mathrm{w}}=17 \mathrm{~Hz}$. Die numerischen Anpassungen mit den Parametern der versetzungsfreien Proben führen zu guten Übereinstimmungen mit den Messergebnissen. 

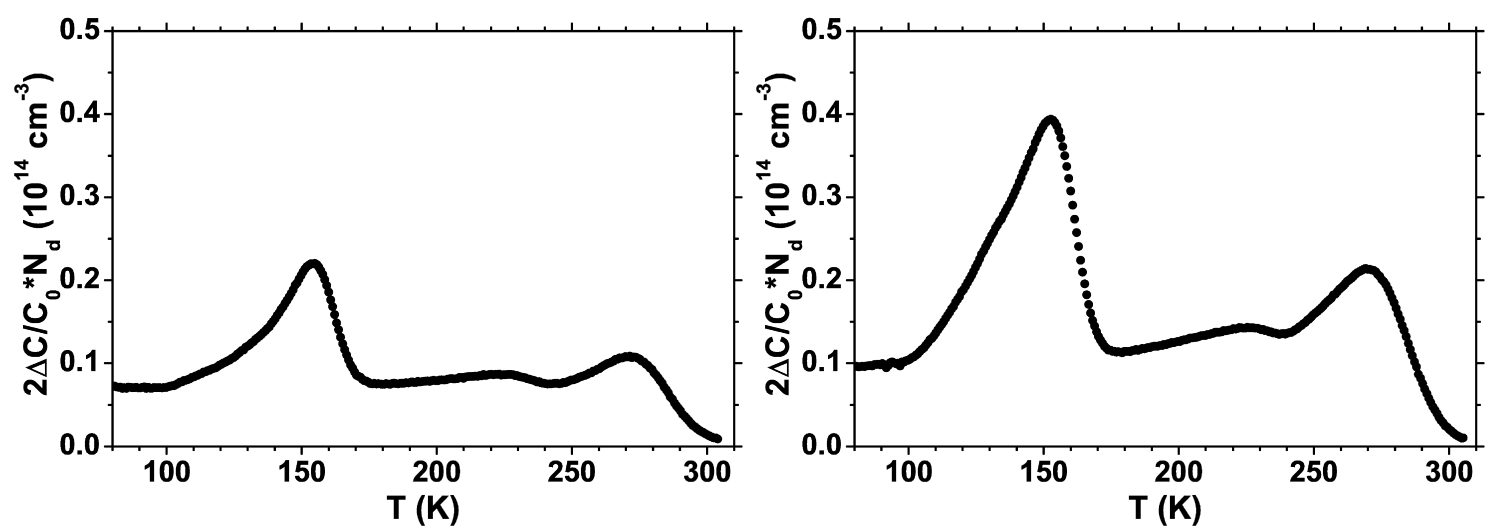

(a) $157 \mu \mathrm{m}$ (links) und $177 \mu \mathrm{m}$ (rechts) zur Golddiffusionsquelle
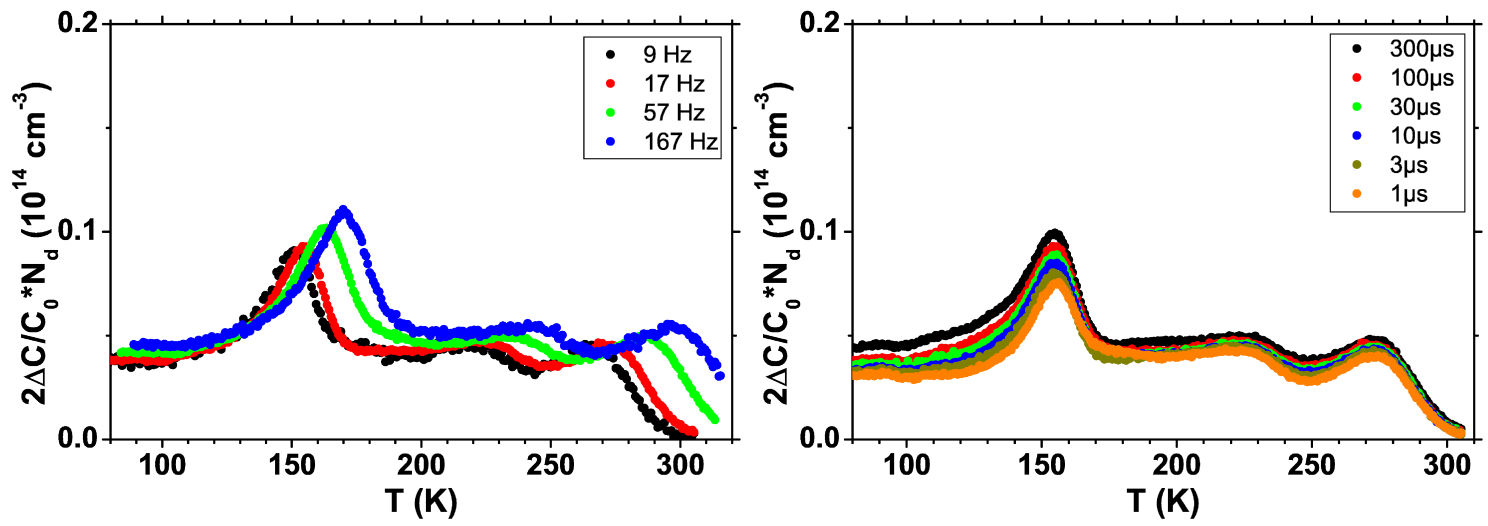

(b) $174 \mu \mathrm{m}$ Abstand zur Golddiffusionsquelle
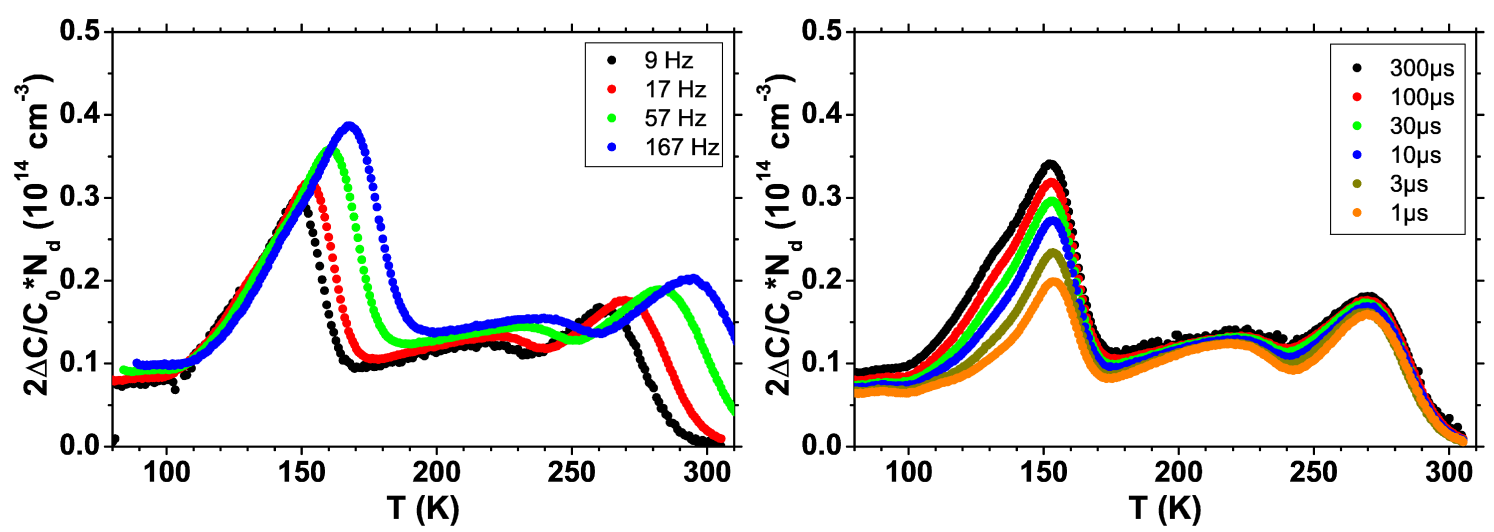

(c) $195 \mu \mathrm{m}$ Abstand zur Golddiffusionsquelle

Abbildung 4.20 - DLTS-Spektren der golddotierten Probe mit der Versetzungsdichte $3.3 \cdot 10^{8} \mathrm{~cm}^{-2}$, a) gemessen bei $\left.t_{\mathrm{p}}=300 \mu \mathrm{s}, f_{\mathrm{w}}=17 \mathrm{~Hz}, b, c\right)$ gemessen in der Frequenzvariation mit $t_{\mathrm{p}}=100 \mu \mathrm{s}$ und in der Pulslängenvariation mit $f_{\mathrm{w}}=17 \mathrm{~Hz}\left(U_{\mathrm{b}}=-5 \mathrm{~V}, U_{\mathrm{P}}=4.1 \mathrm{~V}\right)$. Auffällig ist hier die asymmetrische Verbreiterung und die veränderte Einfangkinetik der Linie bei $150 \mathrm{~K}$. 
Die Abb. 4.20 zeigt Spektren, die an Kontakten \#11c bis \#14c gemessen wurden. In diesen Spektren erscheint an der Stelle von $\mathrm{Au}_{\mathrm{p}}$ eine asymmetrisch zu tieferen Temperaturen verbreiterte Linie mit deutlich kleinerer Amplitude.

Darüber hinaus besitzt diese Linie die Einfangcharakteristik eines ausgedehnten Defektes mit lokalisierten Zuständen (siehe Kap. 2.1.1), wie aus der Abb. 4.21, in der die Einfangkinetiken aller Kontakte mit der eines Golddonatorzustandes verglichen werden, hervor geht.

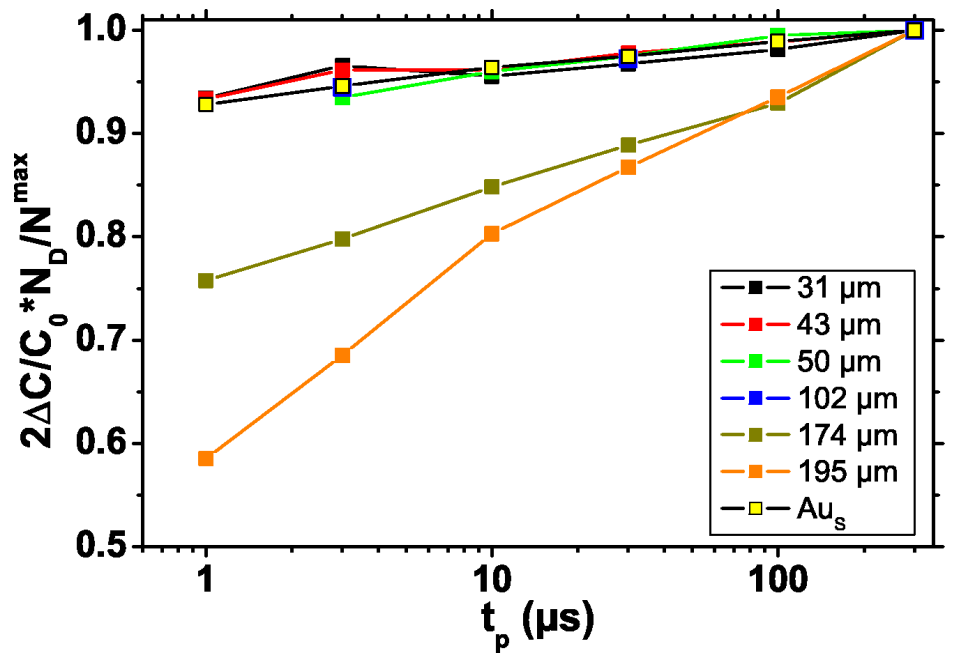

Abbildung 4.21 - Vergleich der Einfangkinetiken der Linie Au $u_{\mathrm{p}}$ mit der eines Golddonatorzustandes in versetzungsfreiem Material. Aufgetragen ist der Wert der Amplitude normiert auf den Wert gemessen bei einer Einfangzeit von $t_{\mathrm{p}}=300 \mu$ s über der Einfangzeit. Nahe der Golddiffusionsquelle entspricht die Einfangcharakteristik der des Gold-

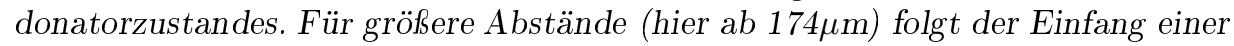
logarithmischen Abhängigkeit von der Einfangzeit $t_{\mathrm{p}}$. Ab hier sind die DLTSLinien asymmetrisch verbreitert.

Während der Einfang mindestens bis zum Kontakt \#8c mit dem Abstand von $102 \mu \mathrm{m}$ zur Golddiffusionsquelle dem des Golddonators in versetzungsfreiem Silizium entspricht, ändert sich dieses ab einem Abstand von frühestens $174 \mu \mathrm{m}$, wobei die asymmetrische Verbreiterung zwischen $140 \mu \mathrm{m}$ und $157 \mu \mathrm{m}$ einsetzt. Somit ist davon auszugehen, dass sich in diesem Bereich auch die Einfangcharakteristik von der eines Punktdefektes zu der eines ausgedehnten Defektes mit lokalisierten Zuständen verändert. Um diese Annahme zu untermauern, sind in der Abb. 4.22 die Transienten gezeigt, die an den Kontakten $\#$ 2c, \#5c, \#9c, \#10c, \#11c und \#13c im Maximum von $\mathrm{Au}_{\mathrm{p}}$ bzw. im Maximum der asymmetrisch verbreiterten Linie gemessen wurden. Bis zu einem Abstand von $140 \mu \mathrm{m}$ zur Golddiffusionsquelle lassen sich die Transienten durch eine Exponentialfunktion anpassen, während die Transienten der tiefer liegenden Kontakte nicht dem Verlauf einer Exponentialfunktion folgen. Demzufolge findet in den Kontakten ab einer Tiefe von 


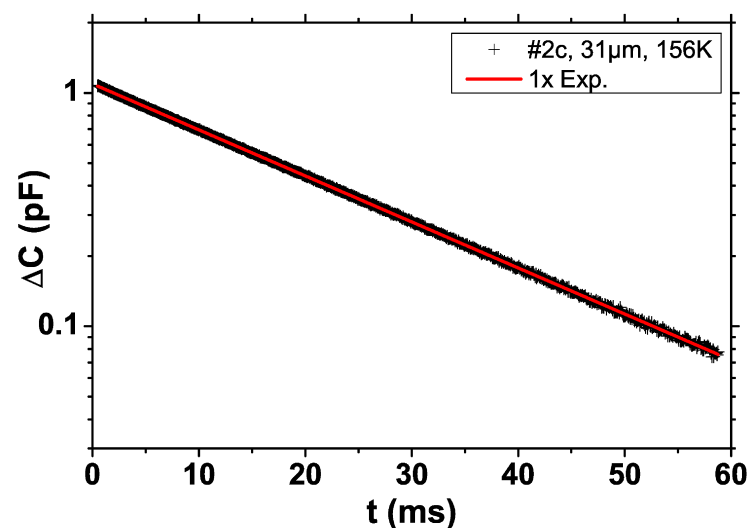

(a) $31 \mu \mathrm{m}$ Abstand zur Golddiffusionsquelle

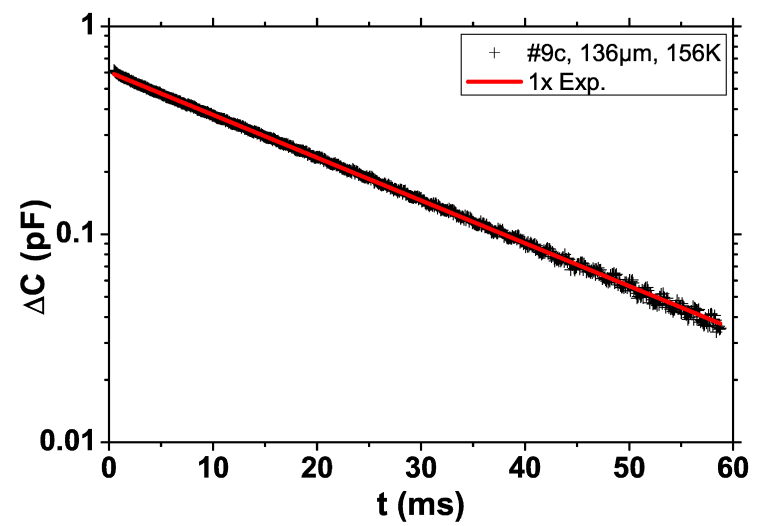

(c) $136 \mu \mathrm{m}$ Abstand zur Golddiffusionsquelle

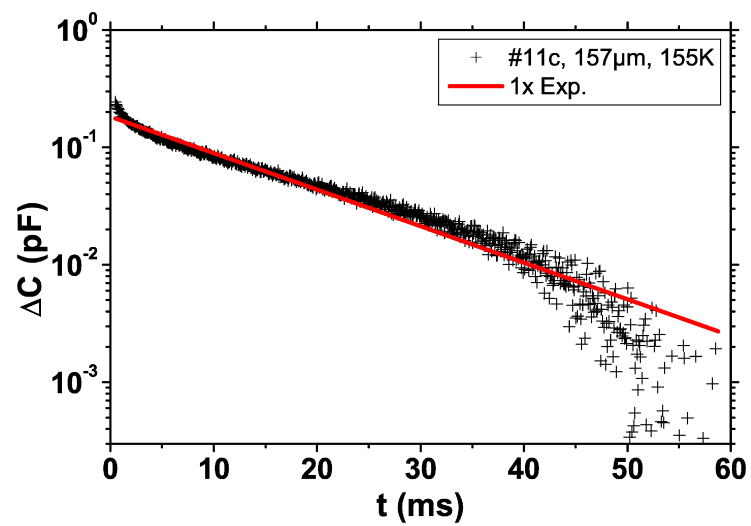

(e) $157 \mu \mathrm{m}$ Abstand zur Golddiffusionsquelle

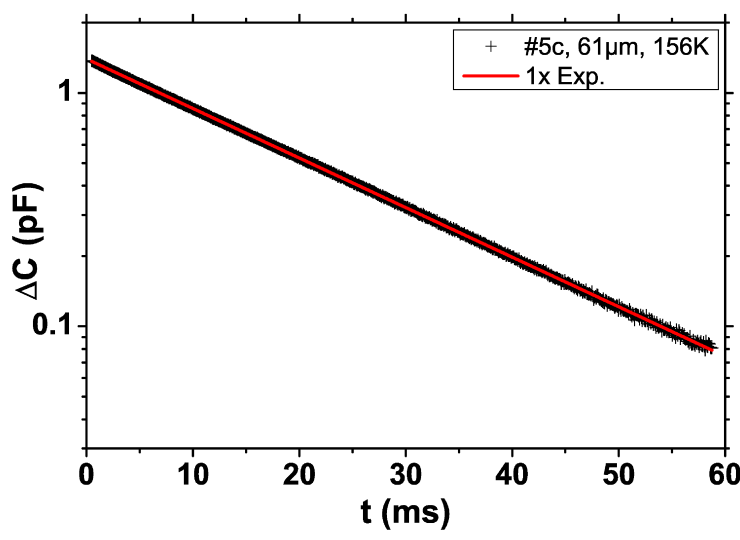

(b) $61 \mu \mathrm{m}$ Abstand zur Golddiffusionsquelle

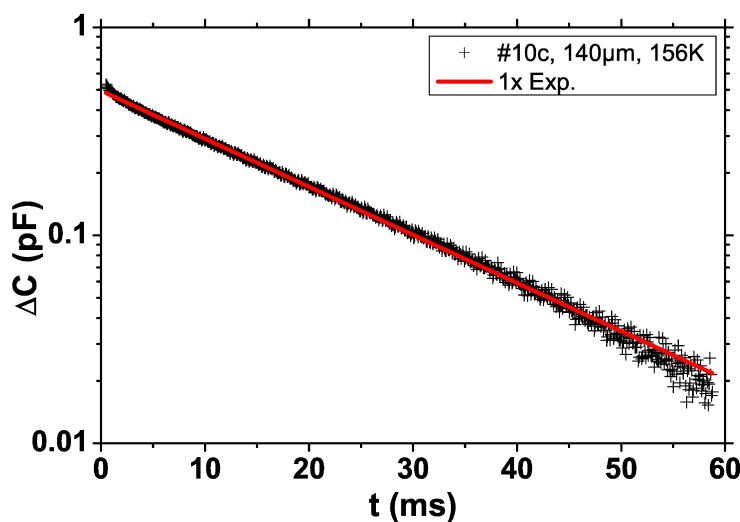

(d) $140 \mu \mathrm{m}$ Abstand zur Golddiffusionsquelle

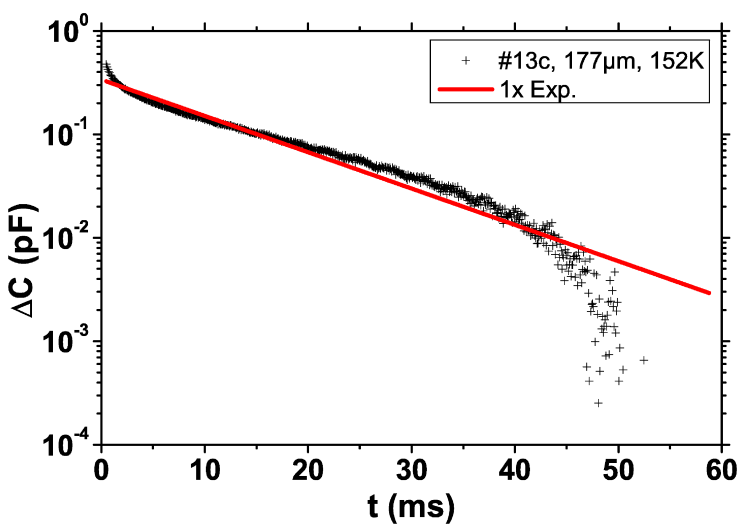

(f) $177 \mu \mathrm{m}$ Abstand zur Golddiffusionsquelle

Abbildung 4.22 - Transienten der Kontakte a) \#2c, b) \#5c, c) \#9c, d) \#10c, e) \#11c und f) \#13c. Die Transienten in a)-d) sind exponentiell und lassen sich durch eine Exponentialfunktion anpassen (Ergebnisse der einfach exponentiellen Anpassungen jeweils als rote Linie). Die an den Kontakten e) \#11c und f) \#13c gemessenen Transienten zeigen einen Verlauf, der nicht durch eine Exponentialfunktion angepasst werden kann. Exponentielle Transienten sind Hinweise auf die Ladungsträgeremission aus dem Zustand eines Punktdefektes. 
$157 \mu \mathrm{m}$ Löcheremission nicht wie bei einem Punktdefekt nur aus einem Energieniveau statt.

Wie aus den Spektren hervor geht, nimmt die Amplitude von $\mathrm{Au}_{\mathrm{p}}$ wie die der Linie $\mathrm{Au}_{\mathrm{n}}$ in der $\mathrm{n}$-dotierten Probe mit der höheren Versetzungsdichte ab einem Abstand von ca. $100 \mu \mathrm{m}$ stark ab. Dieser Umstand wird durch die Abb. 4.23 verdeutlicht. Die

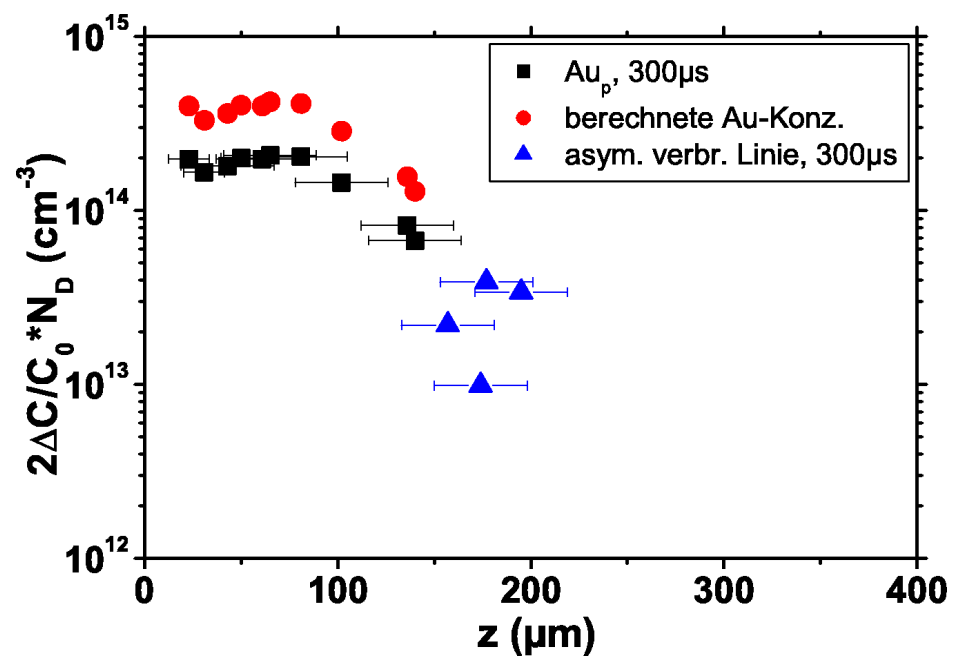

Abbildung 4.23 - Amplitudenwerte der A $u_{\mathrm{p}}$-Linie gemessen bei $t_{\mathrm{p}}=300 \mu$ s über dem Abstand des entsprechenden Kontaktes zur Golddiffusionsquelle. Ab einer Tiefe von ca. $100 \mu \mathrm{m}$ nimmt deren Amplitude signifikant ab. Die Kreise zeigen die aus den Werten der Amplituden berechneten Konzentrationen, die nach dem Modell von Pons [23] berechnet wurden. Die Fehlerbalken zeigen den Bereich, über den aufgrund der Größe des Kontaktes gemittelt wird.

Fehlerbalken zeigen jeweils den Bereich der Probe an, über den aufgrund der Größe des Kontaktes gemittelt wird. Die Kreise stellen die Konzentrationen dar, die mit dem Modell von PONS ${ }^{[23]}$ aus den gezeigten Amplitudenwerten berechnet wurden (Die Einschränkungen dieses Modells sowie die Bedeutung der blauen Linie wurden bereits oben kurz erläutert.). Es fällt auf, dass der maximale Abstand zur Golddiffusionsquelle, an dem $\mathrm{Au}_{\mathrm{p}}$ mit den zur Linie $\mathrm{Au}-\mathrm{D}$ (versetzungsfreies Silizium) vergleichbaren Eigenschaften gemessen werden kann $(140 \mu \mathrm{m})$, etwa mit dem maximalen Abstand übereinstimmt, an dem $\mathrm{Au}_{\mathrm{n}}$ von der C-Linie zu trennen ist $(141 \mu \mathrm{m})$.

Zur Analyse der asymmetrisch verbreiterten Linie werden einige Spektren des Kontaktes \#14c mit den Spektren einer anderen p-dotierten versetzungshaltigen Probe in der Abb. 4.24 verglichen. Die Probe entstammt einer Arbeit von Kveder Und MitarbeiTERN ${ }^{[57.76]}$. Die Versetzungsdichte dieser Probe wird dort mit $(3-5) \cdot 10^{8} \mathrm{~cm}^{-2}$ angegeben. Die Probe wurde nach der Verformung für $30-50$ Minuten bei $820^{\circ} \mathrm{C}$ einem Aluminiumgetterschritt ${ }^{[77]}$ mit einer Abkühlrate von $8 \mathrm{~K} / \mathrm{min}$ unterzogen. Eine Eindiffusion von Gold wurde nicht vorgenommen. Die an unterschiedlichen Proben gemessenen Spektren 

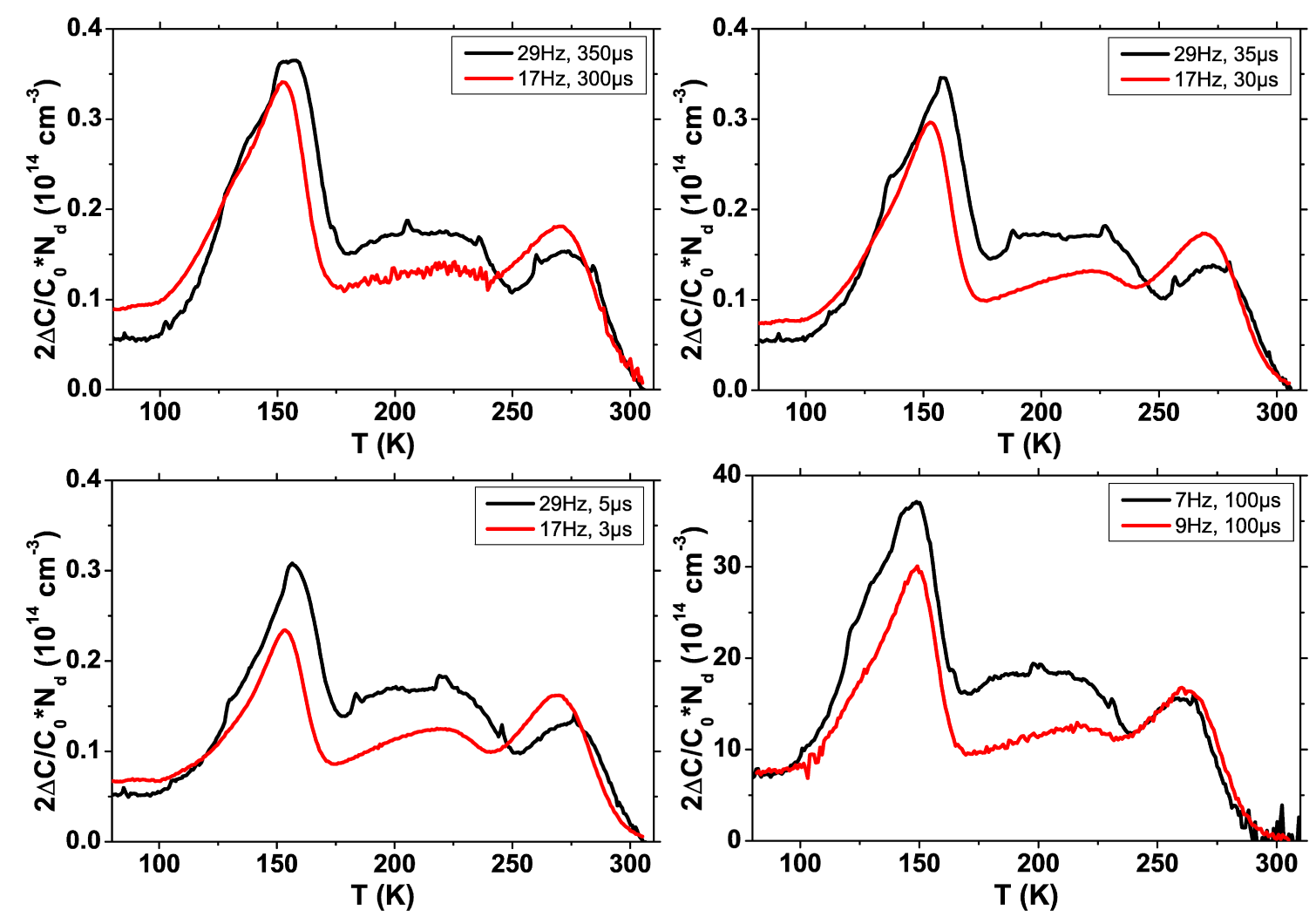

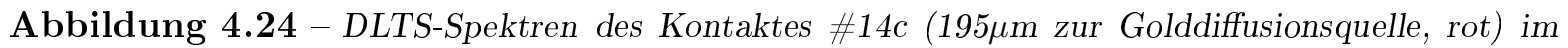
Vergleich mit den Spektren einer anderen p-dotierten versetzungshaltigen Probe (schwarz). Letztere wurde einem Aluminiumgetterschritt nach der Verformung, jedoch keiner Eindiffusion von Gold unterzogen ${ }^{[57.76]}$. Der Vergleich ist ein deutlicher Hinweis darauf, dass es sich bei den DLTS-Spektren jenseits von $150 \mu \mathrm{m}$ um Spektren versetzungsinduzierter Zustände handelt.

gleichen sich in der Anzahl der gemessenen Linien und deren Formen. Die Abweichungen der Temperaturen, bei denen die Linien gemessen werden, lassen sich jeweils mit den leicht abweichenden Frequenzen erklären, mit denen die Spektren gemessen wurden. Diese Übereinstimmungen legen den Schluss nahe, dass es sich bei den Spektren der hier gemessenen Probe, in die Gold eindiffundiert wurde, lediglich um die Spektren von gegetterten versetzungshaltigen p-dotierten Proben handelt. Dass eine Gold-SiliziumPhase, die in dieser Arbeit als Golddiffusionsquelle benutzt wurde, einen signifikanten Gettereffekt zumindest für Kobalt besitzt, konnte SATTLER ${ }^{[77]}$ durch die Messung des Verteilungskoeffizienten von Kobalt in Silizium zur Gold-Silizium-Schmelze nachweisen. Um die Entwicklung der asymmetrischen Verbreiterung in Abhängigkeit vom Abstand zur Golddiffusionsquelle zu zeigen, sind in der Abb.4.25 einige Spektren, die mit der Einfangzeit $300 \mu$ s gemessen wurden, in einem Diagramm dargestellt. Aus dieser Abbildung wird ersichtlich, dass die Emission, die zur asymmetrischen Verbreiterung führt, erst 


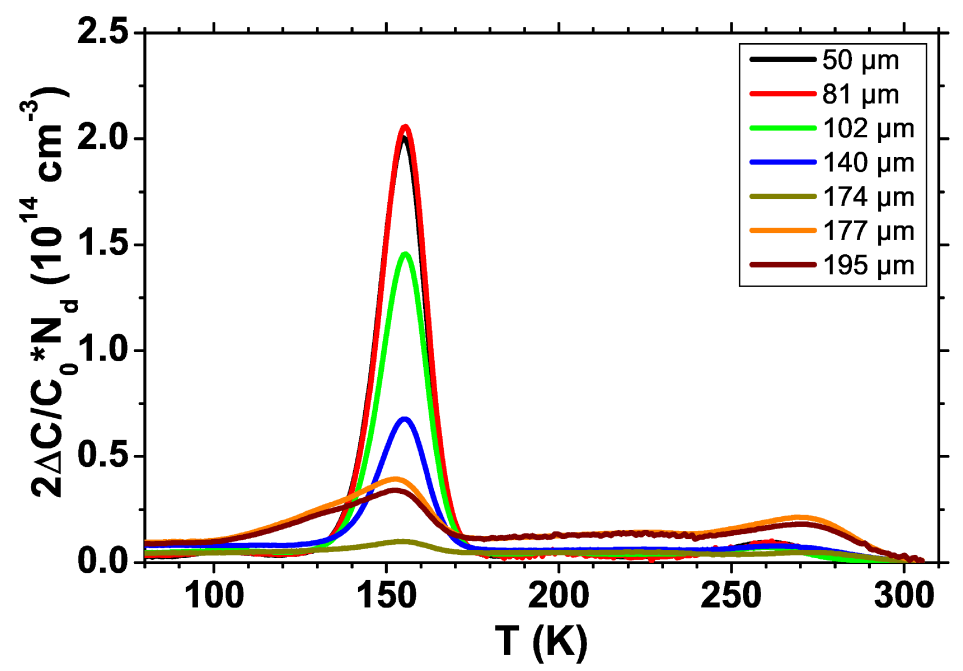

Abbildung 4.25 - Vergleich einiger Spektren, gemessen bei $t_{\mathrm{p}}=300 \mu \mathrm{s}, 17 \mathrm{~Hz}$. Die Emission, die zur asymmetrischen Verbreiterung führt, tritt erst in Erscheinung, wenn die Amplitude von $A u_{\mathrm{p}}$ deutlich reduziert ist. Gleichzeitig gibt es eine Verschiebung und einen Anstieg der Linie im Temperaturbereich zwischen $262 \mathrm{~K}$ und $270 \mathrm{~K}$.

auftritt, wenn die Amplitude deutlich verringert ist. In geringeren Abständen zur Golddiffusionsquelle ist das Signal im Anstieg zur Tieftemperaturflanke kleiner als das Signal, das bei drei der vier Kontakte gemessen wird, bei denen die asymmetrische Verbreite-

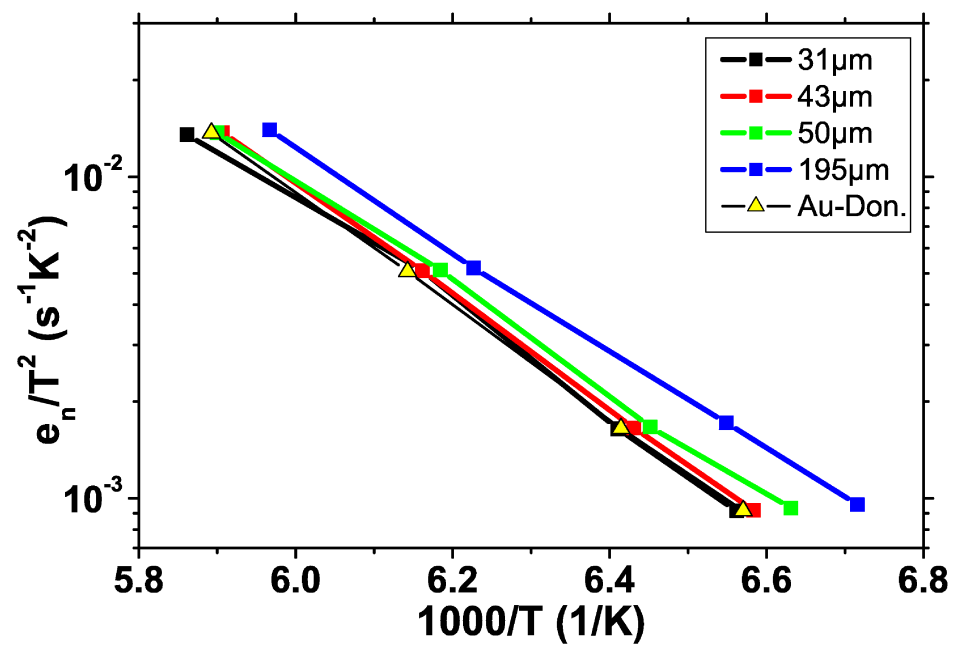

Abbildung 4.26 - Vergleich der thermischen Emissionsraten der Defekte, deren DLTS-Linien in pdotiertem versetzungshaltigem ( $A u_{\mathrm{p}}$, asymmetrisch verbreiterte Linie) und versetzungsfreiem $(A u-D)$ Silizium gemessenen wurden. Der Verlauf der asymmetrisch verbreiterten DLTS-Linie bei $195 \mu \mathrm{m}$ ist zu tieferen Temperaturen verschoben. 
rung auftritt (Kontakte \#11c, \#13c, \#14c). Außerdem verschiebt sich das Maximum der Linie bei $262 \mathrm{~K}$ auf eine Temperatur von $270 \mathrm{~K}$ bei gleichzeitigem Anstieg von deren Amplitude. Diese Verschiebung zeigt, dass es sich dabei um unterschiedliche Defekte handeln muss. Darüber hinaus ist das Messsignal im Temperaturbereich zwischen 180K und $240 \mathrm{~K}$ in den Bereichen um etwa den Faktor 2-3 erhöht, wo die Linie Au $\mathrm{p}_{\mathrm{p}}$ nicht mehr gemessen werden kann, bzw. die asymmetrische Verbreiterung auftritt.

Der Vergleich der Emissionscharakteristiken in Abb. 4.26 zeigt eine gute Übereinstim-

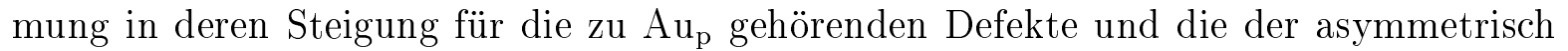
verbreiterten Linie. Allerdings ist der Verlauf der Emissionscharakteristik für die asymmetrisch verbreiterte Linie zu tieferen Temperaturen verschoben.

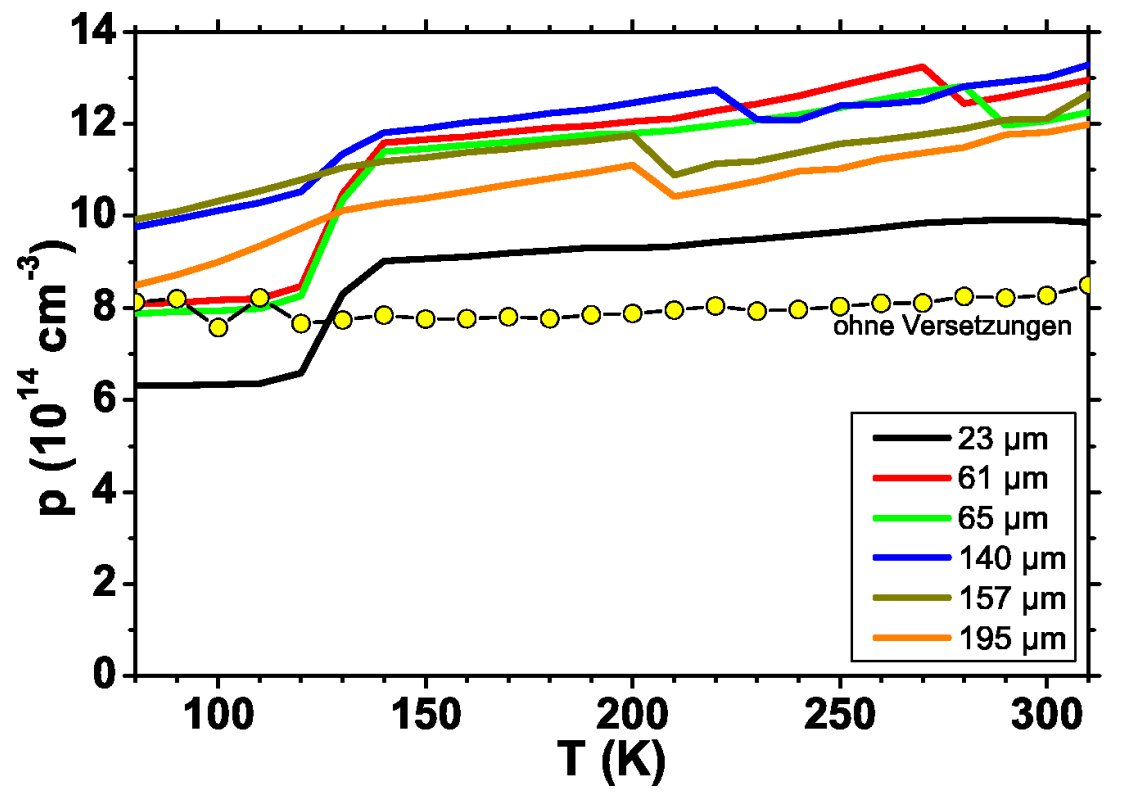

Abbildung 4.27 - Dichte freier Ladungsträger in Abhängigkeit von der Temperatur der p-dotierten versetzungshaltigen Probe. Als Vergleich ist der entsprechende Verlauf für die golddotierte versetzungsfreie Probe dargestellt. Die nominelle Dotierkonzentration betrug $10^{15} \mathrm{~cm}^{-3}$, in der versetzungfreien Probe wurde ein konstanter Wert von $8 \cdot 10^{14} \mathrm{~cm}^{-3}$ gemessen. Aufgrund eines für diese Messungen anzusetzenden Fehlers von 20\% kann keine deutliche Abweichung der freien Löcherdichte von der Dichte der flachen Dotierung beobachtet werden.

Auch an den Kontakten der p-dotierten Probe wurden die temperaturabhängigen Ladungsträgerdichten gemessen (Abb.4.27). Hier gibt es eine Änderung der freien Ladungsträger im Temperaturbereich um $130 \mathrm{~K}$ von 3 bis $4 \cdot 10^{14} \mathrm{~cm}^{-3}$ bis zu einem Abstand von $140 \mu \mathrm{m}$ zur Golddiffusionsquelle. Jenseits dieses Abstandes gibt es eine leichte Änderung über den gesamten Temperaturbereich. Im Vergleich mit dem Verlauf der versetzungsfreien Probe, die aus dem gleichen Ausgangsmaterial wie die versetzungshaltige Probe mit der Nenndotierung $10^{15} \mathrm{~cm}^{-3}$ hergestellt wurde, fällt auf, dass die Ladungsträgerdich- 
te in der versetzungshaltigen Probe über den gesamten Temperaturbereich tendenziell zu höheren Werten verschoben erscheint. Dieser Effekt ist größer als der zu erwartende Fehler von $20 \%$.

\section{Zusammenfassung}

Im folgenden sollen die an p-dotiertem versetzungshaltigem Silizium erzielten Ergebnisse zusammengefasst werden:

1. Die Emissions- sowie die Einfangcharakteristik der $\mathrm{zu} \mathrm{Au}$ gehörenden Defekte decken sich mit denen des Golddonatorzustandes in versetzungsfreiem Silizium.

2. Die Amplitude der DLTS-Linie $\mathrm{Au}_{\mathrm{p}}$ verringert sich mit zunehmendem Abstand zur Golddiffusionsquelle.

3. Die Entfernung zur Golddiffusionsquelle, bei der die Amplitude von $\mathrm{Au}_{\mathrm{p}}$ deutlich abnimmt, stimmt mit der Entfernung überein, bei der die Linie $\mathrm{Au}_{\mathrm{n}} \mathrm{im} \mathrm{n}$-dotierten Silizium mit gleicher Versetzungsdichte noch von der C-Linie zu trennen ist.

4. In diesem Bereich erscheint anstelle von $\mathrm{Au}_{\mathrm{p}}$ eine asymmetrisch zu tieferen Temperaturen verbreiterte Linie, deren Amplitude mit zunehmendem Abstand zur Golddiffusionsquelle nicht in der Weise abfällt, wie die Amplitude von $\mathrm{Au}_{\mathrm{p}}$ in etwas geringerem Abstand. Gleichzeitig ändert sich das Einfangverhalten des Defektes, welches dann dem eines ausgedehnten Defektes mit lokalisierten Zuständen entspricht.

5. Die Spektren, die in diesen Bereichen gemessen werden, zeigen große Übereinstimmungen mit Spektren, die an versetzungshaltigem und aluminiumgegetterten p-dotierten Proben gemessen wurden, die in der Versetzungsdichte in etwa übereinstimmen und in die kein Gold eindiffundiert wurde.

6. Die Emission, die zumeist zur asymmetrischen Verbreiterung führt, findet nicht bei den Kontakten statt, die in geringeren Abständen zur Golddiffusionsquelle liegen.

7. Die Linie des Donatorzustandes des Gold-Wasserstoff-Komplexes $\left(\mathrm{AuH}^{(+/ 0)}\right)$ konnte nicht gemessen werden, obwohl dies gemäß der Kontaktpräparation zu erwarten gewesen wäre.

8. Die Linie im oberen Temperaturbereich verschiebt sich bei kleinerer zu erwartender Goldkonzentration zu höheren Temperaturen bei gleichzeitigem Anstieg der Amplitude.

9. Im Temperaturbereich um 140K gibt es einen Sprung in der Dichte freier Ladungsträger für solche Kontakte, an denen die Linie $\mathrm{Au}_{\mathrm{p}}$ gemessen werden kann. 
10. Im Vergleich mit dem Verlauf einer versetzungsfreien Probe gleichen Ausgangsmaterials erscheinen die Ladungsträgerdichten der versetzungshaltigen Probe über den gesamten Temperaturbereich zu höheren Werten verschoben. 


\subsection{Goldausscheidungen}

Die Untersuchung von Probenbereichen mit hoher Goldkonzentration mittels TEM erfolgte zum einen, um eventuelle Goldausscheidungen, wie sie RodRIGUEz UND MitARBEITER ${ }^{[20]}$ in ihren versetzungshaltigen Proben im Abstandsbereich unter $100 \mu \mathrm{m}$ von der Golddiffusionsquelle nachgewiesen haben, zu untersuchen. Zum anderen kann für den Fall, dass Gold im Versetzungskern segregiert, ein Kontrast am Ort des Versetzungskerns in hochaufösenden TEM-Aufnahmen erwartet werden, da Gold ein deutlich höheres Streupotential für Elektronen besitzt als die umgebenen Siliziumatome.

Abb. 4.28(a) zeigt die TEM-Aufnahme mehrerer Versetzungsabschnitte und eines Teilchens. Dass es sich bei diesem Teilchen um eine Goldausscheidung handelt, konnte mittels einer EDX-Messung nachgewiesen werden (Abb. 4.29). Die Dichte der im Querschnitt

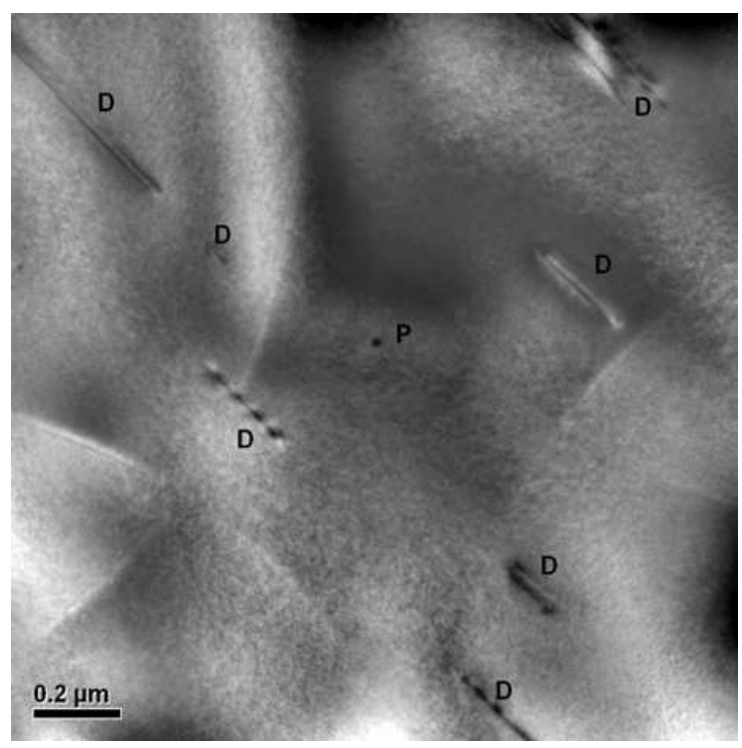

(a) Versetzungen (D) und eine Goldausscheidung (b) $(\mathrm{P})$.

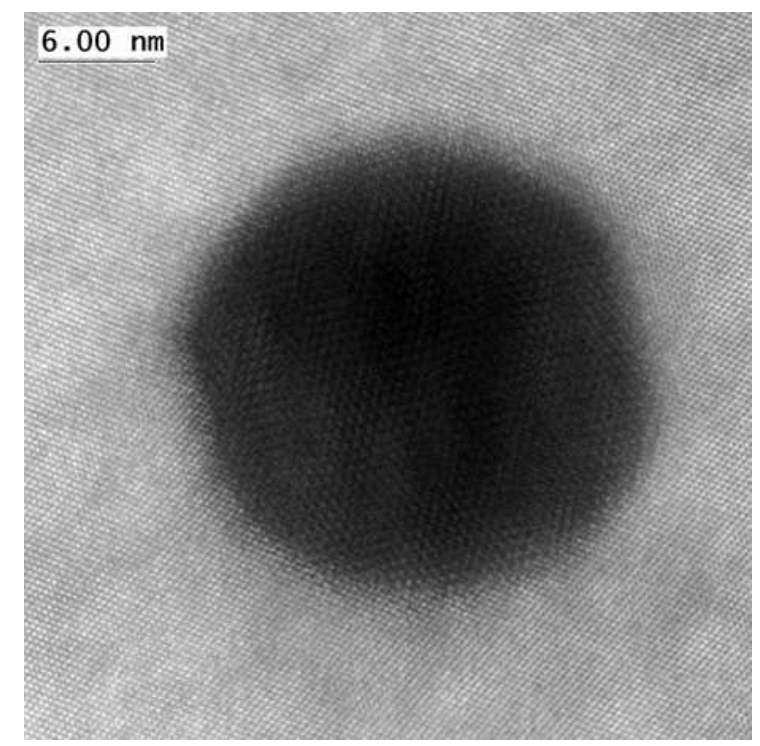

(b) Goldausscheidung mit einem Durchmesser von $24 \mathrm{~nm}$.

Abbildung 4.28 - TEM-Aufnahmen im Abstand zwischen 30 und 40 $\mu \mathrm{m}$ von der Golddiffusionsquelle. a) Mehrere Versetzungsabschnitte und eine Goldausscheidung. Die Dichte der Ausscheidungen wurde auf $10^{11} \mathrm{~cm}^{-3}$ abgeschätzt., b) Goldausscheidung in hochaufgelöster TEM.

kreisförmigen Goldausscheidungen wurde auf $10^{11} \mathrm{~cm}^{-3}$ abgeschätzt. Die bei RoDRIGUEz Und MitaRBeITERN gefundenen Goldausscheidungen, die unter vergleichbaren Bedingungen erzeugt worden sind, hatten ebenfalls einen kreisförmigen Querschnitt. Mit einem durchschnittlichen Durchmesser von etwa 20nm lässt sich unter der Annahme, dass die Ausscheidungen sphärisch sind und dass es sich bei diesen Ausscheidungen um Gold und nicht um ein metastabiles Goldsilizid handelt, die in den Ausscheidungen enthaltene Ge- 


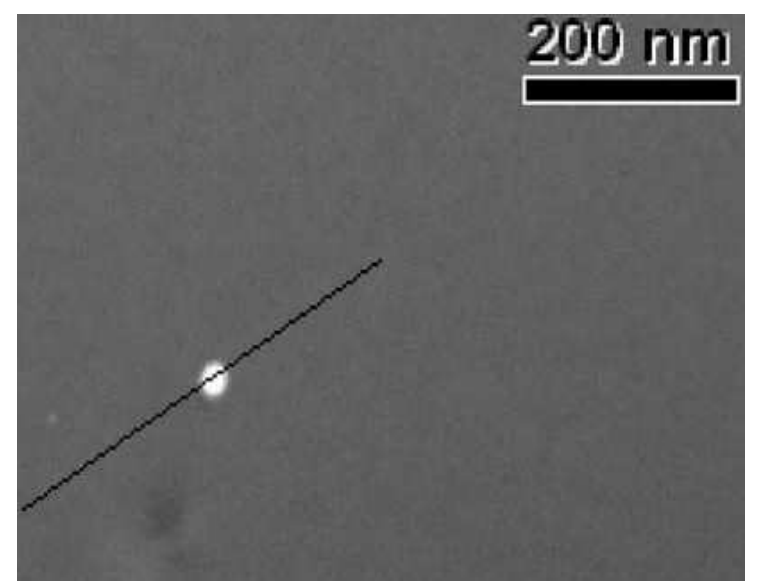

(a) Linienabtastung eines Teilchen nahe der Golddiffusionsquelle.

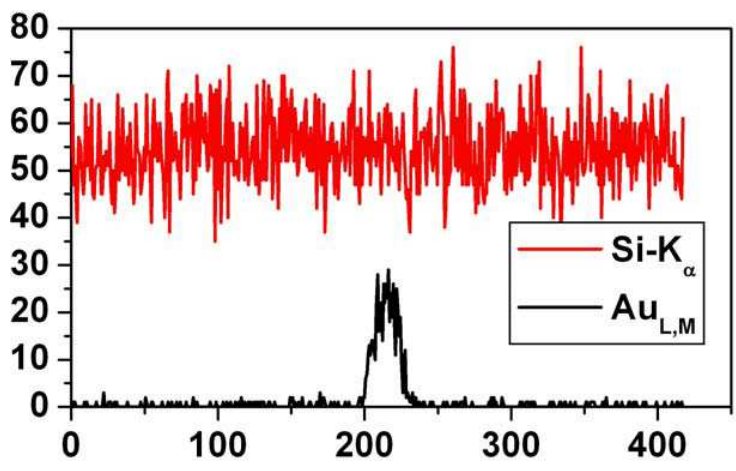

(b) EDX-Spektrum des Teilchens aus Bild a).

Abbildung 4.29 - Ergebnis der EDX-Messung an einem in der Nähe der Golddiffusionsquelle gefundenen Teilchens, a) Darstellung der abgetasteten Linie, mit dem Teilchen, b) EDX-Spektrum der in a) gezeigten Linie.

samtdichte auf $5 \cdot 10^{16} \mathrm{~cm}^{-3}$ berechnen. Hierbei handelt es wegen der zweiten Annahme um eine obere Grenze, da nicht klar ist, ob es sich wie bei BAUMANN UND SCHRÖTER ${ }^{\text {[38] }}$ um ein Goldsilizid handelt.

Anders als in Kap. 2.2.2 beschrieben, sind diese Ausscheidungen nicht während der Abkühlung entstanden, da die Probe, wie in Kap. 3.4 beschrieben, abgeschreckt wurde, sondern wie bei RoDriguez Und MitarbeITERN während der Eindiffusion des Goldes. 


\section{Diskussion}

In diesem Kapitel soll untersucht werden, welche Schlussfolgerungen über die Wechselwirkung von Gold und Versetzungen aus den im Kap. 4 beschriebenen experimentellen Ergebnisse gezogen werden können. Es ergibt sich ein konsistentes Bild der elektrischen Eigenschaften und der von RODRIGUEZ UND MITARBEITERN ${ }^{[20]}$ berichteten Löslichkeits- und Diffusionsdaten von Gold in versetzungshaltigem Silizium. Im ersten Abschnitt werden dazu die dominierenden Linien in den DLTS-Spektren der versetzungshaltigen Proben, $\mathrm{Au}_{\mathrm{n}}$ und $\mathrm{Au}_{\mathrm{p}}$, dem Akzeptorzustand $\mathrm{Au}^{(0 /-)}$ bzw. dem Donatorzustand $\mathrm{Au}^{(+/ 0)}$ substitutionell gelöster Goldatome zugeordnet. Insbesondere wird ausgeschlossen, dass die beobachteten DLTS-Signale Goldatomen im Versetzungskern zuzuordnen sind.

Im zweiten Teil soll gezeigt werden, dass die Besetzung dieser Zustände mit Ladungsträgern die Dichte freier Ladungsträger signifikant beeinflusst. Darüber hinaus wird gezeigt, dass eine zunehmende Gesamtgoldkonzentration in Verbindung mit Versetzungen zu einer Absenkung der Dichte freier Elektronen in den n-dotierten Proben führt. Eine Erklärung dafür wird ein tiefer Akzeptorzustand sein, der aufgrund dieser Abhängigkeit an den Versetzungen zu lokalisieren ist.

Ein Vorgriff auf das Ergebnis des Kap. 5.3, in dem eine alternative Deutung der Ergebnisse von RODRIGUEZ UND MITARBEITERN ${ }^{[20]}$ vorgeschlagen wird, erlaubt dann die Berechnung der Liniendichte des Akzeptorzustandes und damit der Gesamtkonzentration des im Versetzungskern gebundenen Goldes. Mit diesen Ergebnissen kann das Auftreten des besagten Akzeptorzustandes mit den Goldatomen im Versetzungskern korreliert werden.

Im darauf folgenden Abschnitt wird die experimentell beobachtete Einfangkinetik des Goldakzeptorzustandes $\mathrm{Au}^{(0 /-)}$ untersucht. Dazu werden zwei Modelle diskutiert, die die Grenzfälle der Wechselwirkung innerhalb der geladenen Zustände entlang des Versetzungskerns beschreiben. Damit und mit den zuvor berechneten Linienladungen können die Energieniveaus der neutralen, golddekorierten Versetzungen abgeschätzt werden. Schließlich wird die Frage zu klären sein, warum dieser Akzeptorzustand des Goldes im Versetzungskern kein auffälliges Signal im DLTS-Spektrum hinterlässt. Nicht berücksichtigt wird der Einfluss des Deformationspotentials auf die im Verzerrungsfeld gebundenen Goldatome (vgl. Kap. 2.4.2). Wie im Anhang B gezeigt wird, befindet sich dort nur ein zu vernachlässigender Anteil der Goldatome.

Anschließend wird kurz auf die offensichtlich von der Versetzungsdichte abhängige Golddiffusion eingegangen. Allerdings kann der Einfluss der Versetzungen mit den Er- 
gebnissen dieser Arbeit nicht geklärt werden. Die Möglichkeit, dass der unterschiedliche mittlere Abstand der Versetzungen die Einstellung von Konzentrationsgleichgewichten beeinflusst, wird dort diskutiert.

Im letzten Abschnitt wird kurz der Zusammenhang zwischen der Abnahme der Konzentration substitutionellen Goldes und der gleichzeitigen Zunahme versetzungsinduzierter DLTS-Linien in der p-dotierten Probe diskutiert.

\subsection{Elektrische Eigenschaften golddekorierter Versetzungskerne}

\subsubsection{Identifikation der goldinduzierten DLTS-Linien}

In dem Kap. 4.2 .2 sind die Eigenschaften der DLTS-Linien $A u_{n}$ und $A u_{p}$ ausführlich untersucht worden. Dort wurde folgendes festgestellt:

- Die zur DLTS-Linie $\mathrm{Au}_{\mathrm{n}}$ gehörenden Defekte stimmen sowohl in den Emissionseigenschaften als auch in der Linienbreite mit den Eigenschaften der Linie $\mathrm{Au}+\mathrm{G} 4$ (Kap.4.2.1.1) gehörenden Defekten in versetzungsfreiem Silizium überein (vgl. z.B. Abb. 4.16 und 4.4 sowie 4.13).

- In der Einfangkinetik gibt es deutliche Unterschiede zwischen den zur Linie $\mathrm{Au}_{\mathrm{n}}$ und $\mathrm{Au}+\mathrm{G} 4$ gehörenden Defekten (Abb. 4.15).

- Die zur Linie $\mathrm{Au}_{\mathrm{p}}$ gehörenden Zustände zeigen sowohl die gleichen Emissionseigenschaften und die gleiche Linienbreite wie die zur Linie Au-D gehörenden Defekte in versetzungsfreiem Silizium (vgl. z.B. Abb. 4.7 und 4.19(c) sowie 4.26). Darüber hinaus stimmen diese Defekte auch in ihren Einfangkinetiken überein (4.21).

Die Übereinstimmungen legen den Schluss nahe, dass es sich bei den DLTS-Linien $A u_{n}$ und $\mathrm{Au}_{\mathrm{p}}$ um die DLTS-Linien der Zustände $\mathrm{Au}^{(0 /-)}$ und $\mathrm{Au}^{(+/ 0)}$ des substitutionell gelösten Goldes handelt. Wie in Kap. 2.2.3 beschrieben, besitzen substitutionelle Goldatome in Silizium zwei tiefe Zustände in der Bandlücke: Einen Akzeptorzustand $\mathrm{Au}^{(0 /-)}$ (vgl. Kap. 4.2.1), der aufgrund seiner Lage in der Mitte der Bandlücke im DLTS-Spektrum von n-dotiertem und in dem von in p-dotiertem Silizium jeweils mit einer Linie verknüpft ist, und einen Donatorzustand $\mathrm{Au}^{(+/ 0)}$ in der unteren Hälfte der Bandlücke. Diese sind zusammen mit den Zuständen des Gold-Wasserstoff-Komplexes noch einmal in der Abb. 5.1 dargestellt:

Aus dem Vergleich der Ergebnisse in versetzungsfreiem und versetzungshaltigem Silizium geht hervor, dass ein Einfluss der Versetzungen auf die Einfangkinetik nur in n-dotiertem Material nachgewiesen werden kann (vgl. Abb. 4.15 und 4.21). Allerdings muss darauf hingewiesen werden, dass der Einfangquerschnitt des Golddonatorzustandes 


$$
\begin{gathered}
E_{\mathrm{L}} \\
\frac{--}{=} E_{\mathrm{L}}-0.18 \mathrm{eV} \\
E_{\mathrm{L}}-0.51 \mathrm{eV} \frac{-}{0} \\
E_{\mathrm{V}}+0.32 \mathrm{eV} \frac{0}{+} E_{\mathrm{L}}-0.6 \mathrm{eV} \\
E_{\mathrm{V}} \frac{\frac{0}{+} E_{\mathrm{V}}+0.2 \mathrm{eV}}{\mathrm{Au} \quad \mathrm{AuH}}
\end{gathered}
$$

\begin{abstract}
Abbildung 5.1 - Bandschema der goldinduzierten Defekte, $E_{\mathrm{L}}, E_{\mathrm{V}}$ : Leitungs-, Valenzbandkante, $A u, A u H:$ substitutionelles Gold und Gold-Wasserstoff-Komplex mit den dazugehörigen Donator- und Akzeptorzuständen. In versetzungshaltigem Silizium können fast keine Gold-Wasserstoff-Komplexe nachgewiesen werden. Für die Diskussion der Einfangkinetiken in versetzungshaltigem Silizium sei darauf hingewiesen, dass der Einfangquerschnitt des $\mathrm{Au}^{(+/ 0)}$ für Majoritätsladungsträger um ca. zwei Größenordnungen höher ist als der des $A u^{(0 /-)}$.
\end{abstract}

$\mathrm{Au}^{(+/ 0)}$ für den Locheinfang um ca. zwei Größenordnungen größer ist als der des entsprechenden Akzeptorzustandes $\mathrm{Au}^{(0 /-)}$ für den Elektroneneinfang (vgl. Tab. 4.3). Analoge Aussagen für die mit Gold-Wasserstoff-Komplexen verknüpften DLTS-Linien sind aus folgenden Gründen nicht möglich:

- In den Spektren versetzungshaltigen, p-dotierten Siliziums lässt sich keine Linie des $\mathrm{AuH}^{(+/ 0)}$ nachweisen. Offensichtlich hemmen Versetzungen die Bildung von Gold-Wasserstoff-Komplexen. Es ist bekannt, dass Wasserstoff versetzungsindu-

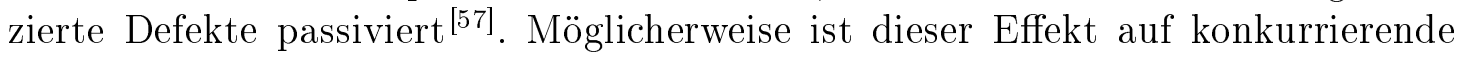
Reaktionen des Wasserstoffs mit gold- und versetzungskorrelierten Defekten zurückzuführen.

- Im n-dotierten Silizium können die Linien des $\mathrm{Au}^{(0 /-)}$ und des $\mathrm{AuH}^{(0 /-)}$ nicht getrennt werden. Somit können auch keine Einfangkinetiken der einzelnen Defekte gemessen werden.

- Darüber hinaus scheint die Dichte der Gold-Wasserstoff-Komplexe im versetzungshaltigen, n-dotierten Silizium so klein zu sein, dass sich deren DLTS-Linie des Doppelakzeptorzustandes $\mathrm{AuH}^{(-/-)}$für die meisten in diesem Material gemessen Spektren nicht deutlich von der A-Linie abhebt. Die A-Linie ist typisch für das DLTSSpektrum versetzungshaltigem, $\mathrm{n}$-dotierten Siliziums (vgl. Kap. 2.4.2, 4.2.2.1). In einigen Spektren scheint die Linie des $\mathrm{AuH}^{(-/-)}$über der A-Linie zu dominieren 
(Abb. 4.13(a), 4.13(c). Temperaturbereich um 105K). Allerdings besitzt die Amplitude der zugehörigen DLTS-Linie aufgrund des kleinen Einfangquerschnitts von $\mathrm{AuH}^{(-/-)}$auch in versetzungsfreiem Silizium eine deutliche Abhängigkeit von der Einfangzeit (vgl. Abb. 4.4(a)), was die Beurteilung des Einflusses der Versetzungen auf die Einfangkinetik deutlich erschwert.

Es bleibt festzuhalten, dass die Einfangkinetik des Goldakzeptorzustandes $\mathrm{Au}^{(0 /-)}$ in versetzungshaltigem Silizium von der in versetzungsfreiem Silizium abweicht. Der zugehörige Donatorzustand $\mathrm{Au}^{(+/ 0)}$ zeigt diesen Effekt nicht. Allerdings muss hier dessen vergleichsweise großer Einfangquerschnitt berücksichtigt werden. Für die Zustände des Gold-Wasserstoff-Komplexes kann ein solcher Effekt nicht nachgewiesen werden, da offensichtlich deren Bildung durch Versetzungen gehemmt wird.

\subsubsection{Einfluss der Besetzung tiefer Zustände auf die Dichte freier Ladungsträger}

Im folgenden Abschnitt soll gezeigt werden, dass die Umladung der Zustände des substitutionell gelösten Goldes in den versetzungshaltigen Proben, deren Konzentration im Kap. 4.2.2 bestimmt wurden, zu kompensatorischen Effekten in den Dichten $n$ bzw. $p$ der freien Ladungsträger geführt haben. Darüber hinaus wird ein Absenken dieser Dichte in den n-dotierten Proben mit Akzeptorzuständen an den Versetzungen in Verbindung gebracht.

Zunächst sollen einige Kriterien der Bewertung der in den Abb. 4.18 und 4.27 gezeigten Verläufe beschrieben werden: Wie im Kap. 4.2.2.1 erwähnt, muss bei den Absolutwerten der dort gezeigten Dichten freier Ladungsträger ein Fehler von ca. 20\% aufgrund des Fehlers bei der Bestimmung des Kontaktdurchmessers angesetzt werden. Wenn daher wie in Abb. 4.18(a) die Kurven der versetzungshaltigen Proben die der versetzungsfreien schneidet, ist dies nicht auf die Änderung von einem Akzeptor- zu einem Donatorverhalten der Versetzungszustände zurückzuführen. Zudem ist auch der an die Kapazitätskennlinien angepasste Parameter mit einem statistischen Fehler behaftet, mit dem einzelne unsystematische „Sprünge“ in den Verläufen zu begründen sind. Insbesondere kann dieser bei den Aluminiumkontakten der p-dotierten Proben, die eine dünne isolierende Siliziumoxidschicht erfordern (siehe Kap. 3.6), zu derartigen Schwankungen führen.

Aus der Abb. 4.18 ist abzulesen, dass es in den DLTS-Spektren, in denen die Linie des Goldakzeptorzustandes $\mathrm{Au}^{(0 /-)}$ gemessen werden konnte, einen deutlichen Anstieg der freien Elektronenladung $n$ im Bereich um $210 \mathrm{~K}$ gibt. Es soll zunächst gezeigt werden, dass dieser Anstieg auf die Emission der Ladungsträger aus dem $\mathrm{Au}^{(0 /-)}-$ Zustand während der Kapazitätsmessung zurückzuführen ist, auf deren Grundlage $n$ gemessen wurde.

Die Zeitkonstante der Kapazitätsmessungen, d.h. die Dauer für die mit konstanter Rate durchgeführte Variation der Sperrspannung von 0 auf 6V, betrug 23s. Die Emissi- 
onsrate des $\mathrm{Au}^{(0 /-)}$ bei $210 \mathrm{~K}$ berechnet sich mit Gl. (2.4) und den Werten aus Tab. 4.2 zu $(23.7 \mathrm{~s})^{-1}$. Dies ist in guter Übereinstimmung mit dem Kehrwert der Zeitkonstanten aus der Kapazitätsmessung. Daraus ist zu schließen, dass dieser Anstieg von $n$ auf die Emission von Elektronen aus den $\mathrm{Au}^{(0 /-)}$ zurückzuführen ist. Zudem wird dieser Anstieg nur in dem Bereich der Probe gemessen, in dem die Amplitude der zugehörigen DLTS-Linie eine entsprechende Konzentration der Zustände erwarten lässt. Auf diese Weise berechnet sich die Dichte der substitutionellen Goldatome in der Probe mit der höheren Versetzungsdichte an den unterschiedlichen Kontakten $\mathrm{zu} 1.6 \cdot 10^{14} \mathrm{~cm}^{-3}$ bis $3.5 \cdot 10^{14} \mathrm{~cm}^{-3}$ und in der Probe mit der geringeren Versetzungsdichte $\mathrm{zu} 1.2 \cdot 10^{14} \mathrm{~cm}^{-3}$ bis $2.9 \cdot 10^{14} \mathrm{~cm}^{-3}$. Der Fehler von $20 \%$ aufgrund der ungenauen Bestimmung des Kontaktdurchmessers kommt in der Berechnung dieser relativen Abweichungen nicht zum Tragen. Die Werte liegen etwas tiefer als die in Abb. 4.14 gezeigten, berechneten Werten. Allerdings kann aus der Einfangkinetik der zugehörigen Akzeptorzustände $\mathrm{Au}^{(0 /-)}$ (Abb. 4.15) geschlossen werden, dass diese zumindest in der DLTS nicht vollständig umgeladen werden. Der Grund für die systematische Abweichung der hier berechneten Konzentrationen von denen aus den DLTS-Spektren berechneten Werten könnte sein, dass diese Zustände vermutlich auch während der Kapazitätsmessung nicht vollständig umgeladen werden.

In der p-dotierten Probe tritt ein deutlicher Anstieg der freien Löcherdichte $p$ im Temperaturbereich um $130 \mathrm{~K}$ in den Bereichen der Probe auf, in denen die Linie $\mathrm{Au}_{\mathrm{p}} \mathrm{im}$ DLTS-Spektrum gemessenen werden konnte. Mit den Werten aus Tab. 4.3 berechnet sich die Emissionsrate bei $125 \mathrm{~K}$ zu $(9.8 \mathrm{~s})^{-1}$. Auch hier gibt es eine gute Übereinstimmung mit dem Kehrwert der Zeitkonstanten aus der Kapazitätsmessung und es liegt der Schluss nahe, dass dieser Anstieg auf die Lochemission aus dem $\mathrm{Au}^{(+/ 0)}$-Zustand zurückzuführen ist. Ebenso wie in den n-dotierten Proben lassen sich Dichten der substitutionellen Goldatome durch diesen Anstieg bestimmen. Die Dichte nimmt in dieser Probe an den unterschiedlichen Kontakten Werte zwischen $2.2 \cdot 10^{14} \mathrm{~cm}^{-3}$ und $4.3 \cdot 10^{14} \mathrm{~cm}^{-3}$ an. Diese Konzentrationen sind in guter Übereinstimmung mit den aus den Amplituden der DLTS-Linien berechneten Konzentrationen substitutioneller Goldatome (vgl. 4.23).

Neben dem deutlichen Anstieg von $n$ bei $210 \mathrm{~K}$ in den n-dotierten Proben scheint diese Dichte in der Probe mit der höheren Versetzungsdichte in Bereichen nahe der Golddiffusionsquelle über den gesamten Temperaturbereich zu kleineren Ladungsträgerdichten verschoben. Wird diese Verschiebung für Temperaturen oberhalb von 210K ausgewertet, bei denen der Goldakzeptorzustand $\mathrm{Au}^{(0 /-)}$ mit hoher Rate Elektronen emittiert und somit in der Kapazitätsmessung nicht mehr erscheint, so berechnet sich für den Kontakt bei $37 \mu \mathrm{m}$ eine Abnahme von ca. $5 \cdot 4 \cdot 10^{14} \mathrm{~cm}^{-3}$. Dieser Effekt ist mit einer Abnahme in Höhe von ca. $2.2 \cdot 10^{14} \mathrm{~cm}^{-3}$ in der Probe mit der geringeren Versetzungsdichte in $34 \mu \mathrm{m}-$ Entfernung zur Golddiffusionsquelle nicht so stark ausgeprägt und liegt in der Nähe der Fehlergrenze $\left(10^{15} \mathrm{~cm}^{-3} \pm 20 \%\right.$, siehe Kap. 4.2.2.1). Diese Reduktion von $n$ über den gesamten beobachteten Temperaturbereich ist ein Hinweis darauf, dass es einen tiefen Akzeptorzustand geben muss. Dieser Akzeptorzustand hat eine Emissionsrate, die im gesamten Temperaturbereich der DLTS-Messung kleiner als etwa $(23 \mathrm{~s})^{-1}$ sein muss, da 
23s die Zeitkonstante der Kapazitätsmessung war.

Wichtig für die folgende Interpretation ist auch, dass die Abnahme der Dichte freier Ladungsträger vom Abstand zur Golddiffusionsquelle abhängig ist. Genauer gesagt tritt dieser Effekt dort auf, wo auch die Ladungsträgeremission aus den Zuständen des substitutionellen Goldes gemessen wird. Hier gibt es offensichtlich einen Zusammenhang zwischen der Konzentration des Goldes und der Konzentration des Akzeptorzustandes, die für Abb. 5.2 aus den zugehörigen Reduktionen der freien Elektronendichten entnommen wurden.

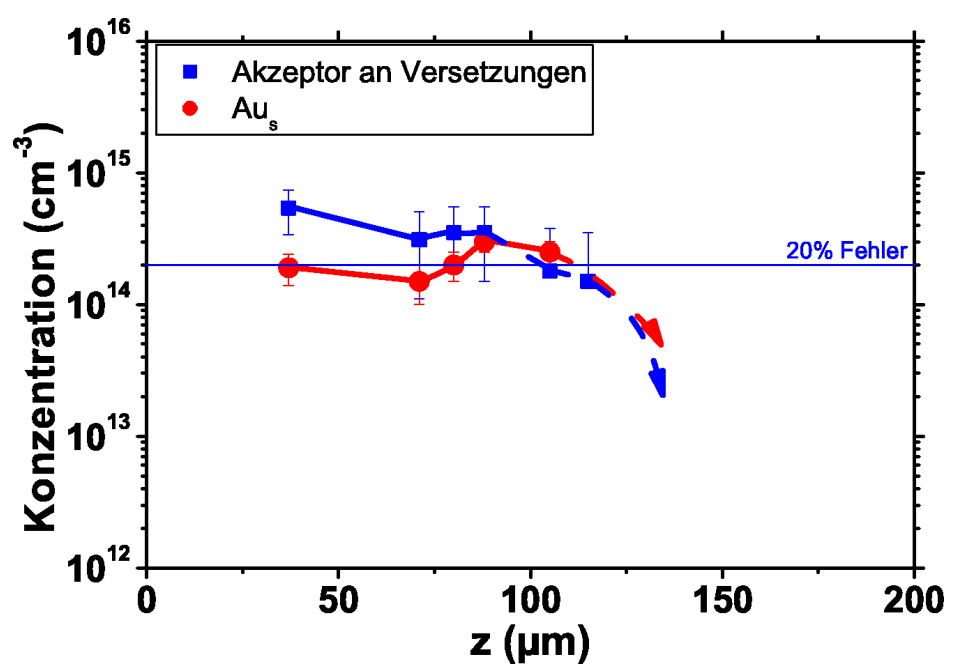

Abbildung 5.2 - Konzentration des tiefen Akzeptorzustandes und der berechneten Konzentration substitutionell gelöster Goldatome der Probe mit der größeren Versetzungsdichte. Der tiefe Akzeptorzustand wird nur dort gemessen werden, wo auch substitutionelles Gold gemessen werden kann. Die Konzentrationen wurden den zugehörigen Abnahmen der freien Elektronendichten entnommen.

Da die Reduktion der freien Ladungsträgerdichte mit größerer Versetzungsdichte zunimmt, liegt es nahe, diesen Akzeptorzustand an den Versetzungen zu lokalisieren. Die aus den Kapazitätsmessungen abgeleitete maximale Emissionsrate dieses Akzeptors von $(23 \mathrm{~s})^{-} 1$ bei $310 \mathrm{~K}$ ist konsistent damit, dass eine zugehörige DLTS-Linie in n-dotiertem Silizium nicht auftritt, da die detektierbaren Emissionsraten oberhalb von $21 \mathrm{~Hz}$ bei $310 \mathrm{~K}$ liegen. Eine von Fujita und Mitarbeitern [15] berechnete Energie für den Akzeptorzustand eines im Versetzungskern gebundenen Goldatoms zwischen $0.57 \mathrm{eV}$ und $0.74 \mathrm{eV}$ (vgl. Abb. 2.7) unterhalb der Leitungsbandkante würde dieser Forderung genügen.

Abb. 4.27 zeigt das Ergebnis der Messung von der Dichte $p$ freier Löcher an der p-dotierten, versetzungshaltigen Probe. Die Dotierung des Siliziummaterials war mit $10^{15} \mathrm{~cm}^{-3}$ angegeben, jedoch wurde in der versetzungsfreien Probe ein über den gesamten Temperaturbereich konstanter Wert von ca. $p=8 \cdot 10^{14} \mathrm{~cm}^{-3}$ gemessen. Demgegenüber scheint die Dichte $p$ für die versetzungshaltigen Proben zu höheren Werten verschoben. 
Dass dieser Anstieg auch für Kontakte gemessen wurde, in deren Spektrum die DLTSLinie des Zustands des substitutionell gelösten Goldes (hier: $\mathrm{Au}_{\mathrm{p}}$ ) nicht mehr auftritt, unterscheidet die p-dotierte von den n-dotierten Proben. Wenn diese veränderte Dichte $p$ also auf Akzeptorzustände zurückzuführen ist, die in der p-dotierten Probe als flacher Akzeptor zusätzliche Ladungsträger zur Verfügung stellt, so muss dieser Akzeptor auch in den Probenbereichen mit kleiner Konzentration substitutioneller Goldatome auftreten. Zumindest kann festgehalten werden, dass eine Reduktion der freien Ladungsträgerdichte in der p-dotierten Probe nicht vorliegt. Daraus folgt der Schluss, dass es keinen Donatorzustand an den Versetzungen vergleichbar zu dem oben beschriebenen Akzeptorzustand gibt, der in dem beobachteten Temperaturbereich freie Ladungsträger binden könnte. FUJITA UND MitARBEITERN ${ }^{[15]}$ berechnen die Energie des Donatorzustandes eines im Versetzungskern gebundenen Goldatoms zu $0.61 \mathrm{eV}$ bis $0.7 \mathrm{eV}$ (vgl. Abb. 2.7). Diese lägen deutlich über dem Fermi-Niveau ( $\approx 0.1 \mathrm{eV}$ oberhalb der Valenzbandkante), wären geladen und würden somit die Dichte freier Ladungsträger reduzieren. Diese Beobachtung wird hier nicht gemacht.

Schließlich kann noch ein Anstieg der Ladungsträgerdichten in den versetzungshaltigen Proben mit zunehmender Temperatur beobachtet werden. Dies gilt insbesondere für die Messungen an den Stellen, wo kein substitutionell gelöstes Gold mehr anzutreffen ist (jeweils bei einem Abstand von etwa $140 \mu \mathrm{m}$ zur Golddiffusionsquelle). Dieser Anstieg ist vermutlich der Emission aus den versetzungsinduzierten Defekten geschuldet, die nicht mit dem Gold in Verbindung stehen. Zu diesen Defekten gehören zum einen die der C-Linie in n-dotiertem Silizium. Zum anderen sind dies die Defekte in p-dotiertem Silizium, die die Linien und Messsignale in den Spektren der Abb. 4.24 verursachen. Legt man die in großen Tiefen $(>140 \mu \mathrm{m})$ der Proben mit hohen Versetzungsdichten gemessenen Verläufe zugrunde, so kann die Dichte der an den Versetzungszuständen gebundenen Ladungen zu etwa $2-4 \cdot 10^{14} \mathrm{~cm}^{-3}$ bestimmt werden.

\subsubsection{Identifikation, Besetzung und Linienladung der Versetzungszustände}

Im vorhergehenden Kapitel wurde gezeigt, dass die tiefen Akzeptorzustände an den Versetzungen liegen müssen. In der Probe mit der höheren Versetzungsdichte konnte zudem eine signifikante Konzentration des tiefen Akzeptorzustandes nur bei einer deutlichen Konzentration substitutionellen Goldes gemessen werden. In der Probe mit der geringeren Versetzungsdichte ist dieser Zusammenhang nicht gegeben. Im Kap. 5.4 wird auf diesen Befund eingegangen.

Mit den Ergebnissen aus dem Kap. 5.3 kann angenommen werden, dass ein erheblicher Teil der Goldatome an den Versetzungen gebunden ist. Somit kann der tiefe Akzeptorzustand mit den Goldatomen entlang der Versetzungen in Verbindung gebracht werden. Dieser Zustand wird im folgenden mit $\mathrm{Au}^{\mathrm{V}}$ bezeichnet. 
Zunächst soll die Liniendichte der Akzeptorzustände $\mathrm{Au}^{\mathrm{V}}$ entlang der Versetzung berechnet werden. Die Resultate aus Kap. 5.3 bieten dann die Möglichkeit, die Liniendichte der im Versetzungskern gebundenen Goldatome zu bestimmen. Aus diesen Ergebnissen kann eine Besetzung der Akzeptorzustände $\mathrm{Au}^{\mathrm{V}}$ bezüglich der Goldkonzentration abgeschätzt werden.

Im Rahmen der hier vorgeschlagenen Deutung der goldinduzierten Akzeptorzustände $\mathrm{Au}^{\mathrm{V}}$ an den Versetzungen kann aus der Abnahme der freien Elektronendichte $n(\approx$ $5.4 \cdot 10^{14} \mathrm{~cm}^{-3}$ in der Probe mit der höheren und $\approx 2.2 \cdot 10^{14} \mathrm{~cm}^{-3}$ in der Probe mit der geringeren Versetzungsdichte, Kap. 5.1.2), der Dichte flacher Donatoren $N_{\mathrm{D}}=10^{15} \mathrm{~cm}^{-3}$ und den Versetzungsdichten die Liniendichte $\kappa$ der besetzten Zustände $\mathrm{Au}^{\mathrm{V}}$ berechnet werden. Die Liniendichte $\kappa$ der Ladungen berechnet sich mit

$$
\kappa=\frac{N_{\mathrm{D}}-n}{N_{\mathrm{d}}}
$$

Für beide Proben mit den unterschiedlichen Versetzungsdichten beträgt $\kappa \approx 1.6 \cdot 10^{6} \mathrm{~cm}^{-1}$ wobei hier die jeweils maximale Absenkung der freien Elektronendichten zugrunde gelegt wurden.

Für die versetzungsinduzierten Defekte, die nicht mit der Dekoration von Gold zusammenhängen (z.B. die die mit den C-Linien assoziierten Defekte), wurde im letzten Kapitel eine Gesamtkonzentration von $\approx 2-4 \cdot 10^{14} \mathrm{~cm}^{-3}$ abgeschätzt. Damit kann eine Liniendichte von besetzten Zuständen für die Proben mit der höheren Versetzungsdichte (p- und n-dotiert) von $0.6-1.2 \cdot 10^{6} \mathrm{~cm}^{-1}$ errechnet werden.

In Kap. ${ }^{[20]}$ wird mit den Ergebnissen der Arbeit von RodRIGUez Und MitARBeITERN ${ }^{[20]}$ eine Bindungsenergie der Goldatome im Versetzungskern von $1.7 \pm 0.2 \mathrm{eV}$ berechnet. Daraus folgt, dass der Besetzungsgrad der Bindungsplätze im Versetzungskern bei der Diffusionstemperatur $800^{\circ} \mathrm{C} \approx 50 \%$ beträgt. Mit der Burgersvektorlänge in Silizium $b=3.84 \AA$ lässt sich eine Liniendichte von Goldatomen entlang des Versetzungskerns von $\approx 13 \cdot 10^{6} \mathrm{~cm}^{-1}$ berechnen. Auf diese Weise ergibt sich ein Besetzungsgrad der goldinduzierten Versetzungszustände $\mathrm{Au}^{\mathrm{V}}$ von $\approx 12 \%$. Die Liniendichte ändert sich nicht, wenn ein Abstand der Bindungsplätze von zwei pro Burgersvektorlänge angenommen wird, da dann die Bindungsenergie von $1.6 \mathrm{eV}$ angenommen werden muss. Damit ändert sich auch der Besetzungsgrad entsprechend.

An dieser Stelle muss darauf hingewiesen werden, dass ein Versetzungskern mit einer derart hohen Besetzung mit Metallatomen keiner konventionellen Beschreibung eines undekorierten Versetzungskernes genügt. Dazu gehört z.B. die Annahme, dass die Bindungsenergie sowie das Energieniveau eines Goldatoms ohne die Berücksichtigung von Wechselwirkungen mit anderen Goldatomen beschrieben werden kann, wie es bei FUJita UND MitarbeiterN ${ }^{[15]}$ der Fall ist. Außerdem kann nicht von einem vollständig rekonstruierten Versetzungskern ausgegangen werden.

Ein Versetzungskern mit einer Linienladung, wie sie hier berechnet wurde, stellt einen 
Vielelektronendefekt im Sinne der Darstellung im Kap. 2.1.2 dar. Dort wird beschrieben, wodurch sich in DLTS-Experimenten die Einfangkinetik typischer Vielelektronendefekte von derjenigen isolierter Punktdefekte unterscheidet.

Die beobachtete Kinetik der Goldakzeptorzustände $\mathrm{Au}^{(0 /-)}$ ist nicht-exponentiell und stets langsamer als der exponentielle Einfang dieses Zustandes in versetzungsfreiem Silizium. Offensichtlich befindet sich ein Teil Goldakzeptorzustände $\mathrm{Au}^{(0 /-)}$ in versetzungshaltigem Silizium im Feld eines negativ geladenen Vielelektronendefektes.

Wie oben beschrieben, kann eine veränderte Einfangkinetik des $\mathrm{Au}^{(+/ 0)}$-Zustandes nicht festgestellt werden. Allerdings besitzt dieser einen Einfangquerschnitt, der um mehr als zwei Größenordnungen größer ist, als der des $\mathrm{Au}^{(0 /-)}$-Zustandes. Es kann also aus der Tatsache, dass der $\mathrm{Au}^{(+/ 0)}$-Zustand diesen Effekt nicht zeigt, nicht unmittelbar geschlossen werden, dass es kein Potential aufgrund positiv geladener Atome in der p-dotierten Probe an den Versetzungen vorliegt. Jedoch ging aus den Betrachtungen über die Absenkungen der freien Ladungsträgerdichten hervor, dass nicht mit positiv geladenen tiefen Donatorzuständen ähnlich den $\mathrm{Au}^{\mathrm{V}}$-Zuständen zu rechnen ist.

\subsubsection{Das Versetzungspotential als Einfangbarriere}

Im folgenden soll qualitativ untersucht werden, ob die nicht-exponentiellen Einfangkinetiken, von denen zwei exemplarisch in Abb. 5.3 mit der Einfangkinetik des Goldakzeptorzustandes in versetzungsfreiem Silizium verglichen werden, durch des Potential einer Linienladung an den Versetzungen erklärt werden können. Darüber hinaus wird aus den Abschätzungen zu den Linienladungsdichten das Potential und das Energieniveau der neutralen Zustände $\mathrm{Au}^{\mathrm{V}}$ berechnet. Dazu werden zwei Grenzfälle der Beschreibung einer Linienladung betrachtet. In dem Modell von READ ${ }^{[48]}$ wird eine vollständige Lokalisierung der Ladungen zugrunde gelegt. Das Modell von VETH und LANOO ${ }^{[78]}$ basiert auf der Annahme einer delokalisierten Ladungsverteilung.

Um die folgenden Betrachtungen besser nachvollziehen zu können, wird zunächst der bisherige Stand der Diskussion zusammengefasst:

- Sowohl in den Spektren der n- als auch in denen der p-dotierten versetzungshaltigen Proben werden Linien der Zustände des substitutionell gelösten Goldes gemessen. Die Einfangkinetik dieser Zustände in den n-dotierten Proben unterliegt dem Einfluss eines elektrostatischen Potentials, während dies für die entsprechenden Zustände der p-dotierten Probe nicht nachgewiesen werden konnte.

- In den n-dotierten Proben konnte eine Linienladungsdichte von $\approx 1.6 \cdot 10^{6} \mathrm{~cm}^{-1}$ aufgrund von tiefen Akzeptorzuständen $\mathrm{Au}^{\mathrm{V}}$ bestimmt werden, die den im Versetzungskern gebundenen Goldatomen zugeordnet werden konnte.

- Für die versetzungsinduzierten Defekte, die nicht mit einer Golddekoration in Verbindung stehen müssen, konnte in gleicher Weise eine Linienladungsdichte von 


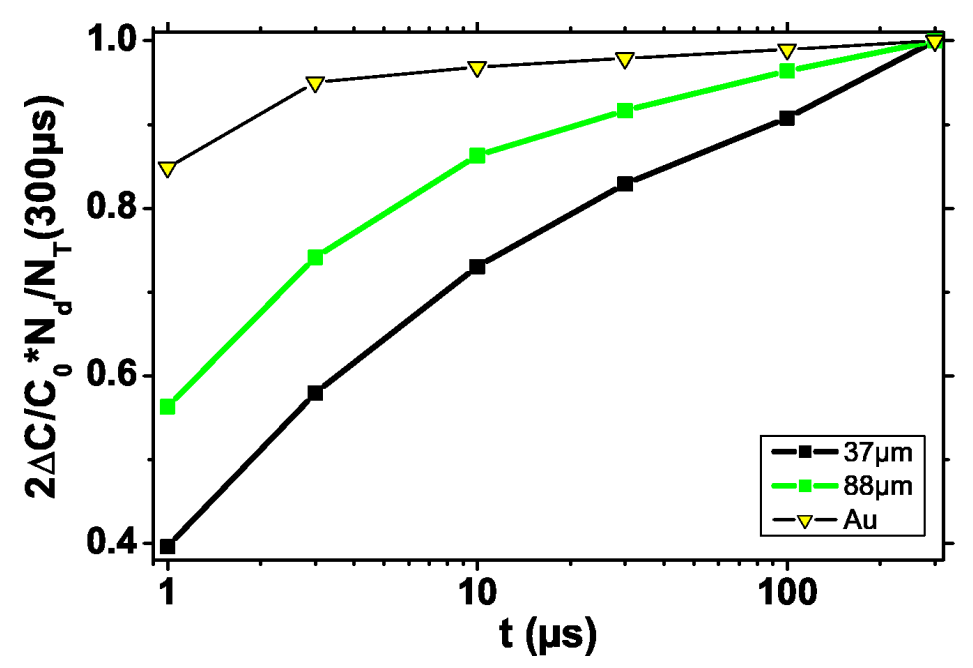

Abbildung 5.3 - Einfangkinetiken des Goldakzeptorzustandes $A u^{(0 /-)}$ der Probe mit der größeren Versetzungsdichte im Vergleich mit Einfangkinetik der aus der versetzungsfreien Probe. Die Einfangrate wird durch Versetzungen deutlich reduziert (vgl. Abb. 4.15).

$0.6-1.2 \cdot 10^{6} \mathrm{~cm}^{-1}$ für die Probe mit der größeren Versetzungsdichte bestimmt werden.

Da diese Linienladungen, wie in Kap. 2.3.2.1 beschrieben, ein zylindersymmetrisches Potential verursachen, das durch die umliegenden ionisierten Dotieratome abgeschirmt wird, liegt die Vermutung nahe, dass dieses Potential die Ursache für die beobachtete veränderte Einfangkinetik in den n-dotierten Proben ist. Der Abschirmradius, der sogenannte „Read-Radius“ $R$ (vgl. Kap. 2.3.2.1), ergibt sich dabei aus der Neutralitätsbedingung für die Ladungsdichten. Der Einfluss des Potentials auf die Einfangkinetik in der DLTS besteht in der Ortsabhängigkeit der Dichte der freien Ladungsträger, die innerhalb des Read-Zylinders mit dem Potential $\Phi$ gemäß $n \cdot \exp \left(\frac{e \Phi}{k_{\mathrm{B}} T}\right)$ zu modifizieren ist. Die Abb. 5.4 verdeutlicht diesen Zusammenhang:

In den n-dotierten Proben gibt es zwei Beiträge für ein solches Potential: Zum einen sind dies die Ladungen, die den besetzten Akzeptorzuständen $\mathrm{Au}^{\mathrm{V}}$ der Goldatome an den Versetzungen zugeschrieben werden. Diese sind, da sie während der DLTS-Messung keine Ladungsträger emittieren (vgl. Kap. 5.1.2) zeitlich konstant. Zum anderen liefern die mit der C-Linie assoziierten Zustände einen Beitrag der allerdings im Vergleich mit den Zuständen $\mathrm{Au}^{(0 /-)}$, in geringem Maße von der Einfangzeit abhängt. Darüber hinaus besteht Grund zu der Annahme, dass die Defekte der C-Linie im Verzerrungsfeld der Versetzung und nicht im Versetzungskern zu lokalisieren sind (vgl. Kap. 2.4.2, ${ }^{[13]}$ ). Da die Ausdehnung des Verzerrungsfeldes (1-2nm $\left.{ }^{[13}\right]$ ) allerdings klein im Vergleich zu $R$ (Größenordnung 100nm) ist, kann bei der Betrachtung des langreichweitigen Potentials von einem Beitrag zur Linienladung gesprochen werden. Insbesondere ist die abschirmende 


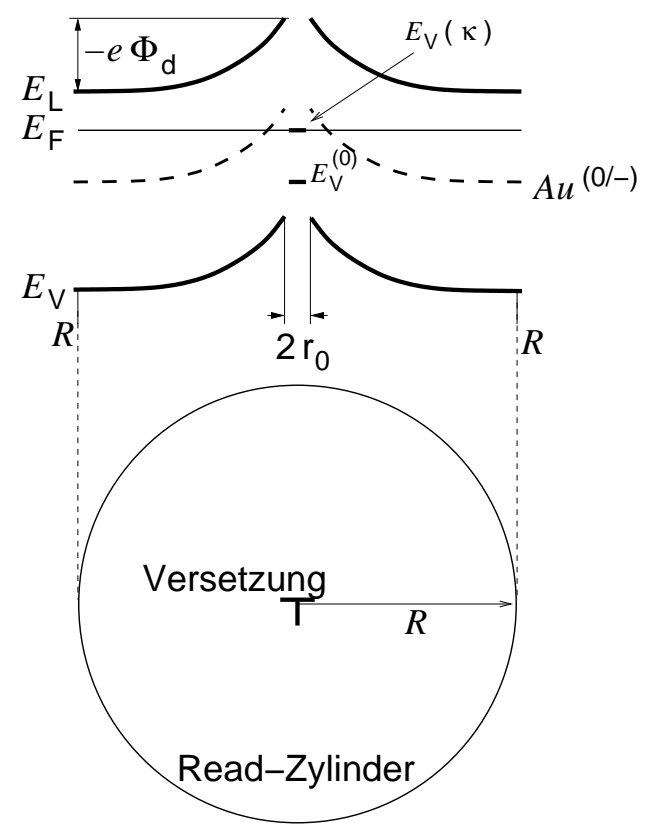

Abbildung 5.4 - Punktdefekt im Potential - eథ einer geladenen Versetzung. Das Energieniveau eines im Volumen gelösten Punktdefektes (hier der $\mathrm{Au}^{(0 /-)}$-Zustand) folgt der Bandverbiegung. Durch das Absenken der Ladungsdichte innerhalb der Potentialzylinders ("Read-Zylinder") mit dem Radius $R$ wird dort der Einfang von Elektronen an den tiefen Zuständen erschwert. ( $E_{\mathrm{V}}, E_{\mathrm{L}}$ : Valenzband-, Leitungsbandkante, $E_{\mathrm{F}}$ : FermiNiveau, $E_{\mathrm{V}}^{(0)}, E_{\mathrm{V}}(\kappa)$ : Energieniveau der neutralen und der mit der Liniendichte $\kappa$ besetzten Versetzung. $r_{0}$ bezeichnet den inneren Abschneideradius.)

Ladungsdichte aus folgendem Grund zeitabhängig: Die Umladung der Goldakzeptorzustände $\mathrm{Au}^{(0 /-)}$ während eines DLTS-Zyklus ändert die Ladungsverteilung innerhalb des Read-Zylinders, die das Potential der Linienladung abschirmt. Dies gilt insbesondere für den in dieser Arbeit vorliegenden Fall, da die Dichte der $\mathrm{Au}^{(0 /-)}$-Zustände nicht klein im Vergleich zur Dichte der flachen Dotierung $N_{\mathrm{D}}$ ist (vgl. Kap. 5.1.2). Somit vergrößert sich der Read-Zylinder während der Einfangphase, weil sich beginnend an dessen Rand die abschirmende Ladungsträgerdichte durch den Einfang von Elektronen an den $\mathrm{Au}{ }^{(0 /-)}$-Zuständen verringert.

Die Einfangkinetik der $\mathrm{Au}^{(0 /-)}$-Zustände lässt sich also wie folgt qualitativ beschreiben: Der Anteil dieser Zustände, der sich während der Einfangphase außerhalb des ReadZylinders befindet, trägt durch eine exponentielle Einfangkinetik wie in versetzungsfreiem Silizium bei (vgl. Gl. (2.11) und Abb. 4.15, Verlauf für Au). Ein anderer Teil der $\mathrm{Au}^{(0 /-)}$-Zustände, der sich während der Einfangphase innerhalb des Read-Zylinders befindet, besitzt eine nicht-exponentielle Einfangkinetik. Die an den tiefen Kontakten der Probe mit der kleineren Versetzungsdichte $(317 \mu \mathrm{m}, 328 \mu \mathrm{m})$ gemessenen DLTS-Linie des Goldakzeptorzustandes $\mathrm{Au}^{(0 /-)}$ ergeben ein dazu konsistentes Bild: Hier gab es keine Ab- 
weichungen der Einfangkinetiken zu denen in den versetzungsfreien Proben (vgl. Abb. 4.15 und $\left.{ }^{[18]}\right)$. Ebenso wenig konnte hier ein Absenken der freien Ladungsträgerdichten aufgrund des Ladungsträgereinfangs an den tiefen Akzeptorzuständen $\mathrm{Au}{ }^{\mathrm{V}}$ beobachtet werden.

In Kap. 5.1.2 konnte anhand der Dichte freier Ladungsträger gezeigt werden, dass es keinen tiefen Donatorzustand an den Versetzungen geben kann, der ähnlich wie der tiefe Akzeptor ein Potential erzeugt. Somit erübrigt sich eine Diskussion der Einfangkinetik des Golddonatorzustandes $\mathrm{Au}^{(+/ 0)}$.

Die in dieser Arbeit gemessene Einfangkinetik der $\mathrm{Au}^{(0 /-)}$-Zustände in versetzungshaltigen Proben kann somit qualitativ insbesondere auf das Potential goldinduzierter Zustände $\left(\mathrm{Au}^{\mathrm{V}}\right)$ an den Versetzungen zurückgeführt werden.

Die mathematische Beschreibung einer solchen Einfangkinetik, die durch ein zeitabhängiges Potential beeinflusst wird, erfordert eine selbst-konsistente Behandlung. Eine solche Behandlung beschreibt PONS ${ }^{[23]}$ für die Umladung tiefer Zustände im Potential der Raumladungszone eines Schottky-Kontaktes. In Fall hoher Konzentrationen tiefer Zustände bewirkt deren Umladung ebenfalls eine Änderung des abschirmenden Potentials. In dieser Behandlung wird die Besetzung der tiefen Zustände iterativ berechnet, und die Poissongleichung mit der veränderten Ladungsverteilung nach jedem Schritt neu integriert. Der Unterschied in dem von Pons behandelten und dem hier vorliegenden Fall des Potentials einer Linienladung besteht darin, dass das Potential der Ladungsverteilung einer abgeschirmten Linienladung aufgrund der Zylindersymmetrie durch räumliche Integration berechnet werden muss.

Im folgenden wird untersucht, wie sich ein derartiger tiefer Akzeptorzustand $\mathrm{Au}^{\mathrm{V}}$ auf des DLTS-Spektrum auswirkt. Maßgeblich dafür ist der Energiezustand des neutralen Defektes, da in der Vorstellung des Starren-Bandmodells die Emissionsrate durch das Energieniveau dieses neutralen Zustandes gegeben ist. Dazu sollen zunächst zwei Grenzfälle der Realisierung einer Linienladung entlang eines Versetzungskernes diskutiert werden. Eines dieser Modelle wurde bereits im einführenden Kap. 2.3.2.1 erklärt. Dabei handelt es sich um ein Modell von READ ${ }^{[48]}$, das eine geladene Versetzung als eine Reihe diskreter Akzeptoren mit einem Energieniveau $E_{\mathrm{V}}^{(0)}$ im neutralen Zustand beschreibt (vgl. Kap. 2.3.2.1). Dabei ist der mittlere Abstand der besetzten Zustände $1 / \kappa(\kappa$ : Liniendichte der Ladungen entlang der Versetzungen). Abb. 5.5(links) verdeutlicht diese Vorstellung. Aufgrund der Annahme eines konstanten mittleren Abstandes geladener Zustände wird dieses Modell als Minimalenergienäherung bezeichnet. Die elektrostatische Verschiebung $E_{\mathrm{V}, \mathrm{R}}(\kappa)-E_{\mathrm{V}, \mathrm{R}}^{(0)}$ beträgt nach diesem Modell (Gl. (2.31), vgl. Abb. 5.4)

$$
E_{\mathrm{V}, \mathrm{R}}(\kappa)-E_{\mathrm{V}, \mathrm{R}}^{(0)}=\frac{e^{2} \kappa}{2 \pi \varepsilon \varepsilon_{0}}\left(\frac{3}{2} \ln \left(\frac{\kappa}{(\pi n)^{1 / 3}}\right)-0.166\right) .
$$

Hier stellen $\varepsilon$ und $\varepsilon_{0}$ die relative Permittivitätszahl bzw. die elektrische Feldkonstante und $n$ die Dichte freier Ladungsträger (hier für den Fall einer n-dotierten Probe, ent- 
sprechend der Abb. (5.4) dar. Über die Bestimmung dieser elektrostatischen Verschiebung kann mit dem Fermi-Niveau die Lage des neutralen Versetzungszustandes berechnet werden.

Die Beschreibung des anderen Grenzfalles stammt von Veth und Lanoo $\stackrel{[78]}{ }$. Hier wurde wiederum eine Reihe äquidistanter Zustände mit dem Abstand $c$ angenommen, die alle die mittlere Linienladung $\overline{1 / \kappa}$ tragen (Abb. 5.5, rechts). In diesem Modell beträgt die elektrostatische Verschiebung

$$
\begin{aligned}
E_{\mathrm{V}, \mathrm{VL}}(\kappa)-E_{\mathrm{V}, \mathrm{VL}}^{(0)} & =\frac{e U \kappa c}{\varepsilon}+\frac{e^{2} \kappa}{2 \pi \varepsilon \varepsilon_{0}}\left(\ln \left(\frac{R}{c}\right)-0.616\right) \mathrm{mit} \\
R & =\sqrt{\frac{\kappa}{\pi n}} .
\end{aligned}
$$

Hier bezeichnet $e U$ die intraatomare Coulombenergie zweier elektrischer Ladungen, die aus der Besetzung eines Atoms im Versetzungskern mit einem weiteren Elektron resultiert. Die Größen $E_{\mathrm{V}}^{(0)}$ stellen in beiden Gleichungen das Energieniveau des neutralen Zustandes dar.
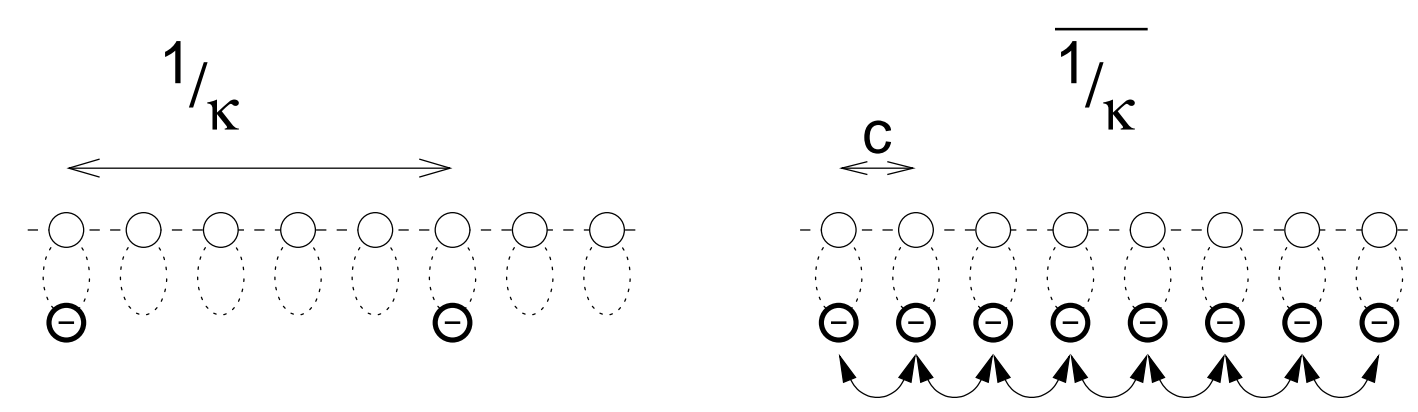

Abbildung 5.5 - Grenzfälle zur Beschreibung geladener Versetzungskernzustände: Links: Modell von READ ${ }^{[48]}$, diskrete Zustände mit einem mittleren Abstand besetzter Zustände von $1 / \kappa$, rechts: Modell von VeTH Und LANoo ${ }^{[78]}$. In diesem Modell haben alle $\mathrm{Zu}$ stände, die den Abstand $c$ voneinander haben, die mittlere Besetzung $\kappa \cdot c$. (Abb. aus $\left.\frac{[13]}{}\right)$

Diese beiden Grenzfälle lokalisierter und bandartiger Zustände werden im Kap. 2.1.2 im Zusammenhang mit den Besonderheiten bezüglich der DLTS-Messungen diskutiert.

Diese Modelle bieten nun die Möglichkeit, die elektrostatische Verschiebung in den beiden Grenzfällen zu berechnen. Dabei soll angenommen werden, dass sich das Potential des mit der C-Linie assoziierten Defekte und das des tiefen Akzeptorzustandes $\mathrm{Au}^{\mathrm{V}}$ summieren. Da die Einfangkinetik der zur C-Linie gehörenden Defekte diese als lokalisiert identifiziert (vgl. Kap. 2.1.2), wird zu Berechnung deren Potentials das Modell von READ herangezogen. Mit den ermittelten Linienladungsdichten $\kappa \approx 0.6-1.2 \cdot 10^{6} \mathrm{~cm}^{-1}$ berechnet sich die elektrostatische Verschiebung aus Gl. (5.2) für die Probe mit der größeren Versetzungsdichte zu 0.03-0.1eV. 
Zur Berechnung des Potentials der tiefen Akzeptorzustände $\mathrm{Au}^{\mathrm{V}}$ werden beide Modelle zur Bestimmung der Grenzfälle verwendet, weil nicht klar ist, ob es sich dabei um lokalisierte oder bandartige Zustände handelt. Als Linienladungsdichte wird der an den oberflächennächsten Kontakten bestimmte Wert $\kappa \approx 1.6 \cdot 10^{6} \mathrm{~cm}^{-1}$ benutzt. Für $U$ wird der von VETH UND LANOO ${ }^{[78]}$ abgeschätzte Wert von $10 \mathrm{~V}$ verwendet. Danach ist $c$ der Abstand zweier Atome entlang des Versetzungskerns, also eine Burgersvektorlänge $3.84 \AA$ A Für die Berechnung des Read-Radius $R$ muss dabei beachtet werden, dass in diesem einfachen Modell die Linienladungen summiert werden müssen, da beide Anteile der Linienladungen abgeschirmt werden. Die auf diese Weise berechneten Werte sind in Tab. 5.1 abzulesen:

\begin{tabular}{|c|c|c|}
\hline & READ & VETH UND LANOO \\
\hline große Versetzungsdichte & $0.14 \mathrm{eV}$ & $0.28 \mathrm{eV}$ \\
\hline kleine Versetzungsdichte & $0.15 \mathrm{eV}$ & $0.3 \mathrm{eV}$ \\
\hline
\end{tabular}

Tabelle 5.1 - Selbstenergien der tiefen Versetzungszustände $A u^{\mathrm{V}}$ nach den Modellen von READ ${ }^{[48]}$ und Veth Und LAnoo ${ }^{[78]}$ für die Proben mit den unterschiedlichen Versetzungsdichten.

Das Fermi-Niveau liegt bei den gegebenen Ladungsträgerdichten und bei der Temperatur von $260 \mathrm{~K}$ bei ca. $0.21 \mathrm{eV}$ unterhalb der Leitungsbandkante. Mit den elektrostatischen Verschiebungen berechnet sich das Niveau der neutralen Versetzungszustände auf etwa $0.35-0.46 \mathrm{eV}$ unterhalb der Leitungsbandkante für den Fall, dass es sich um einen lokalisierten Zustand nach dem Modell von READ handelt. Im anderen Grenzfall der Delokalisierung berechnet sich das Niveau der neutralen Versetzungszustände auf ca. 0.49-0.61eV unterhalb der Leitungsbandkante. Das für den delokalisierten Fall berechnete Energieintervall liegt in guter Übereinstimmung mit dem von FUJITA UND MITARBEITERN ${ }^{[15]}$ ermittelte Energieintervall für den Akzeptorzustand eines im Versetzungskern gebundenen Goldatomes von $0.57 \mathrm{eV}-0.74 \mathrm{eV}$ unterhalb der Leitungsbandkante. Allerdings muss darauf hingewiesen werden, dass bei der Berechnung der Energieniveaus durch FuJITA UND MiTARBEITER nicht berücksichtigt wurde, dass eine ebenfalls in dieser Arbeit berechnete Bindungsenergie zu beträchtlichen Liniendichten von Goldatomen führt (vgl. Kap. 5.3.2). Bei derart großen Liniendichten müssen die Wechselwirkungen der im Versetzungskern gebundenen Goldatome einbezogen werden.

Die Werte des Modells lokalisierter Versetzungskernzustände sind inkonsistent mit der Annahme, dass die Besetzung der tiefen Akzeptorzustände $\mathrm{Au}^{\mathrm{V}}$ über den beobachteten Temperaturbereich der freien Elektronendichte konstant ist (vgl. Kap. 5.1.2).

Nach den bisherigen Überlegungen zum goldinduzierten Akzeptorzustand $\mathrm{Au}^{\mathrm{V}}$ an den Versetzungen stellt sich die Frage, wie sich dieser auf das DLTS-Spektrum auswirkt. Weder im DLTS-Spektrum der n-dotierten noch in dem der p-dotierten versetzungshaltigen Probe lassen sich zunächst Hinweise auf einen Zustand finden, dessen Eigenschaften mit denen des bisher diskutierten Akzeptorzustandes übereinstimmen. Von diesem liegt nun 
eine Abschätzung über dessen Lage in der Bandlücke vor. Ein lokalisierter Defekt im Sinne von READ müsste im DLTS-Spektrum eine Linie mit den Eigenschaften eines ausgedehnten Defektes mit lokalisierten Zuständen (vgl. Kap. 2.1.2) möglicherweise ähnlich der C-Linie erzeugen.

Im anderen Grenzfall des bandartigen Zustandes nach VETH UND LANOO läge dieser in der Mitte bzw. in der unteren Hälfte der Bandlücke. Wenn dieser Zustand also eine Linie im DLTS-Spektrum erzeugt, so müsste diese auch nach Überlegungen zum Absenken der freien Elektronendichte in den Spektren der p-dotierten Probe erscheinen. Dabei muss angemerkt werden, dass diese Linie dann die Eigenschaften eines bandartigen ausgedehnten Defektes zeigen müsste (vgl. 2.1.2). Allerdings sind gerade in den Spektren der p-dotierten Probe, in der aufgrund der Nähe zur Golddiffusionsquelle eine hohe Goldkonzentration an den Versetzungen zu erwarten wäre $(<140 \mu \mathrm{m})$, abgesehen von den schon beschriebenen Linien keine weiteren auffälligen Messsignale erkennbar (vgl. Abb. 4.25). Eine mögliche, spekulative Erklärung wäre, dass sich das DLTS-Singnal einer Löcheremission aus diesem Zustand im DLTS-Spektrum der p-dotierten Proben als Untergrundsignal nicht auflösen lässt. Diese Annahme wäre auch damit zu begründen, dass ein delokalisierter Zustand mit einer sich über einen breiteren Energieintervall erstreckende Zustandsdichte nur ein vergleichsweise kleines DLTS-Signal erzeugen würde. Eine solche Zustandsdichte kann erwartet werden aufgrund der zahlreichen Möglichkeiten von Zuständen, die in der näheren Umgebung eines im Versetzungskern gebundenen Goldatoms Einfluss auf dessen Energiezustand nehmen können (z.B. Siliziumatome, Goldatome, Kinken, Sprünge).

Diese Erklärung wirft folgende Frage auf: Wie Abb. 5.4 zeigt, resultiert das Aufladen der tiefen Akzeptorzustände $\mathrm{Au}^{\mathrm{V}}$ in einer Bandverbiegung, die das Energieniveau des neutralen Zustandes bis auf das Fermi-Niveau erhöht. Im Rahmen der StarrenBand-Näherung folgen dieser Bandverbiegung, abhängig von der Entfernung zum Versetzungskern, alle Zustände innerhalb des Read-Zylinders. Unter der Annahme, dass sich die mit der C-Linie assoziierten Defekte innerhalb eines Radius von 1-2nm um den Versetzungskern befinden (im Verzerrungsfeld), ist zu fordern, dass sich deren Zustände aufgrund dessen zu einem großen Teil oberhalb des Fermi-Niveaus befinden und somit nicht aufgeladen werden können. Dies steht im Widerspruch zu den Ergebnissen der DLTS-Messungen, in denen eine Emission aus diesen Defekten beobachtet wird. Möglicherweise ist dieser Umstand durch den Abstand der Defekte im Verzerrungsfeld zum Zentrum des Potentials zu erklären, was auf dieser Längenskala zu einer relativen Verschiebung der Zustandsdichten führen kann. Außerdem könnten die zugehörigen Zustände an die flachen Bänder im Verzerrungsfeld (vgl. Kap. 2.3.2.2) ankoppeln, was ebenfalls zu einer Verschiebung der Zustände tiefer in die Bandlücke führen würde (ca. 70-80meV).

Als Resultat dieser Betrachtungen kann festgehalten werden, dass die Einfangkinetik des Goldakzeptorzustandes $\mathrm{Au}^{(0 /-)}$ in den versetzungshaltigen Proben konsistent mit der Annahme eines tiefen goldinduzierten Akzeptorzustandes $\mathrm{Au}^{\mathrm{V}}$ an den Versetzungen er- 
klärt werden kann. Die Vorstellung, dass sich bei dem diesem Zustand assoziierten Defekt um Gold im Versetzungskern handelt, wird aus den Untersuchungen im Kap. 5.1.2 und 5.3 gefolgert. Das aus der Beladung dieses Versetzungszustandes resultierende elektrostatische Potential, das die erwähnte Einfangkinetik bewirkt, ist für Konzentrationen tiefer Zustände wie in der vorliegenden Arbeit nur durch einen selbst-konsistenten Ansatz zu berechnen. Die Untersuchungen der Grenzfälle, mit denen eine Linienladung entlang von Versetzungskernen beschrieben werden kann, führt ebenfalls zu einem konsistenten Bild bezüglich theoretischer Untersuchungen aus der Literatur und den Untersuchungen der freien Elektronendichten. Allerdings können nur Vermutungen darüber angestellt werden, warum der tiefe Akzeptorzustand $\mathrm{Au}^{\mathrm{V}}$ an den Versetzungen kein auffälliges Signal in den DLTS-Spektren hinterlässt.

\subsection{Diskussion anderer Modelle zur Beschreibung der modifizierten Einfangkinetik in versetzungshaltigem Silizium}

In diesem Abschnitt soll kurz auf die Ergebnisse und Interpretationen anderer Arbeiten eingegangen werden, die sich mit den elektrischen Eigenschaften von Gold in versetzungshaltigem Silizium beschäftigen.

In ${ }^{[56,60]}$ wurden ebenfalls die elektrischen Eigenschaften von Gold in versetzungshaltigem Silizium mit DLTS untersucht. Die Ergebnisse, die allerdings ausschließlich an n-dotiertem Silizium erzielt wurden, entsprachen im wesentlichen den Ergebnissen in dieser Arbeit. Dort wurden die Einfangkinetiken als das Resultat der Akkumulation von Goldatomen im Verzerrungsfeld der Versetzungen und einer daraus resultierenden besetzungsabhängigen Einfangbarriere interpretiert. Allerdings können mit einer solchen Akkumulation mit einer Bindungsenergie von $0.85 \mathrm{eV}$ (vgl. Kap. 2.4.1) keine Gesamtkonzentrationen erklärt werden, wie sie Rodriguez Und Mitarbeiter ${ }^{[20]}$ in ihrer Arbeit mit der Neutronenaktivierungsanalyse messen. Dieses wird im Anhang B erläutert. Dem widersprechen außerdem die Ergebnisse dieser Arbeit für p-dotierte Proben, die zeigen, das die Einfangbarriere nicht aus dem Aufladen der Goldatome selbst resultieren kann.

In ${ }^{[79]}$ werden Ergebnisse von DLTS-Messungen sowohl von n- als auch von p-dotierten versetzungshaltigen Proben, in die Gold eindiffundiert wurde, dargstellt. Die dort gezeigten Ergebnisse für die n-dotierten Proben entsprechen wiederum im wesentlichen den Ergebnissen dieser Arbeit. Die Spektren der p-dotierten Probe enthalten im unteren Temperaturbereich zwei Linien bei 138K und 157K mit logarithmischer Aufladekinetik. Auf der Basis von theoretischen Arbeiten wird argumentiert, dass es sich bei der DLTSLinie bei 138K um die Linie des Donatorzutandes von Goldatomen im Versetzungskern handelt. Die Linie bei $157 \mathrm{~K}$ wird dem Donatorzustand des Goldes zugeordnet, der auch im versetzungsfreien Silizium gemessen wird. Die logarithmische Einfangkinetik wird 
durch die Akkumulation in Punktdefektwolken um die Versetzungen gedeutet, die sich aufgrund des beschleunigten Abbaus der übersättigten interstitiellen Siliziumatome an den Versetzungen ausbilden könnte. Diese Interpretation kann mit den Ergebnissen dieser Arbeit nicht bestätigt werden. Aufgrund der Diffusionsbedingungen (Zeit und Temperatur) tritt an diesen Stellen möglicherweise kein Gold auf. Dann handelt es sich bei diesem um das Spektrum, das in der vorliegenden Arbeit jenseits eines Abstandes von ca. $150 \mu \mathrm{m}$ zur Golddiffusionsquelle gemessen wird.

Das gleiche Argument der abweichenden Einfangkinetik der p-dotierten und der ndotierten Probe greift auch gegen die Annahme, dass die Linien $\mathrm{Au}_{\mathrm{n}}$ und $\mathrm{Au}_{\mathrm{p}}$ den goldinduzierten Defekten im Versetzungskern zuzuordnen sind. Zudem erscheint die Möglichkeit, dass im Versetzungskern gebundenes Gold die gleichen Emissionseigenschaften wie substitutionelles Gold besitzt, unwahrscheinlich, da sich der gebundene Zustand im Versetzungskern von dem auf den substitutionellen Gitterplätzen deutlich unterscheidet (vgl. Kap. 2.4.2).

\subsection{Konzentrationen von Gold in versetzungshaltigem Silizium}

Zu Beginn dieses Abschnitts sollen Überlegungen zu Gleichgewichtszuständen in Systemen mit Versetzungen gestellt werden. Die Existenz von Versetzungen bedeutet, dass sich das betrachtete System nicht in einem Gleichgewichtszustand befindet $\underline{[13]}$.

Der Begriff „Löslichkeit“ beschreibt die Konzentration, die im Gleichgewicht mit einer zweiten Phase einstellt. Insofern kann hier nur von Konzentrationen gesprochen werden, die unter der Annahme lokaler Gleichgewichte und konstanter Versetzungsdichte durch eine Bindungsenergie beschrieben werden können.

Im Mittelpunkt dieses Kapitels steht der Vorschlag einer alternativen Interpretation der Ergebnisse von Rodriguez und Mitarbeitern [20]. Dazu müssen die wichtigsten Ergebnisse der Arbeit und deren Interpretation kurz zusammengefasst werden. Die Kritik an dieser Interpretation und eine Neubewertung der Ergebnisse mit den dabei getroffenen Annahmen werden Gegenstand des zweiten Teils dieses Abschnitts sein.

\subsubsection{Ergebnisse einer anderen Veröffentlichung und deren Interpretation}

Die in Abb. 5.6 gezeigten Konzentrationsprofile aus der Arbeit von RodRIGUEz UnD MitARBEITERN ${ }^{[20]}$ wurden mit der NAA-Methode (Neutronenaktivierungsanalyse) gemessen. Dieses Verfahren basiert auf dem Zerfall von radioaktivem ${ }^{198} \mathrm{Au} \mathrm{zu}{ }^{198} \mathrm{Hg}$. Die schichtweise Messung der Aktivität liefert Aktivitätsprofile, die durch die Messung einer Referenzprobe auf die Gesamtgoldkonzentration umgerechnet werden können. Zur 
Gesamtkonzentration tragen sämtliche Goldspezies bei: interstitiell und substitutionell gelöstes Gold, an den Versetzungen gebundene Goldatome sowie ausgeschiedenes Gold.

Die Konzentrationsprofile wurden nach den aus der Abbildung zu entnehmenden eingestellten Diffusionsparametern (Diffusionszeit und -temperatur) gemessen. Die bei einer Temperatur gemessenen Profile resultieren aus unterschiedlichen Dotierkonzentrationen (Kreis: praktisch undotiert, Quadrat: $[\mathrm{B}]=3 \cdot 10^{19} \mathrm{~cm}^{-3}$, Kreuz: $[\mathrm{B}]=1.7 \cdot 10^{20} \mathrm{~cm}^{-3}$ ). Die
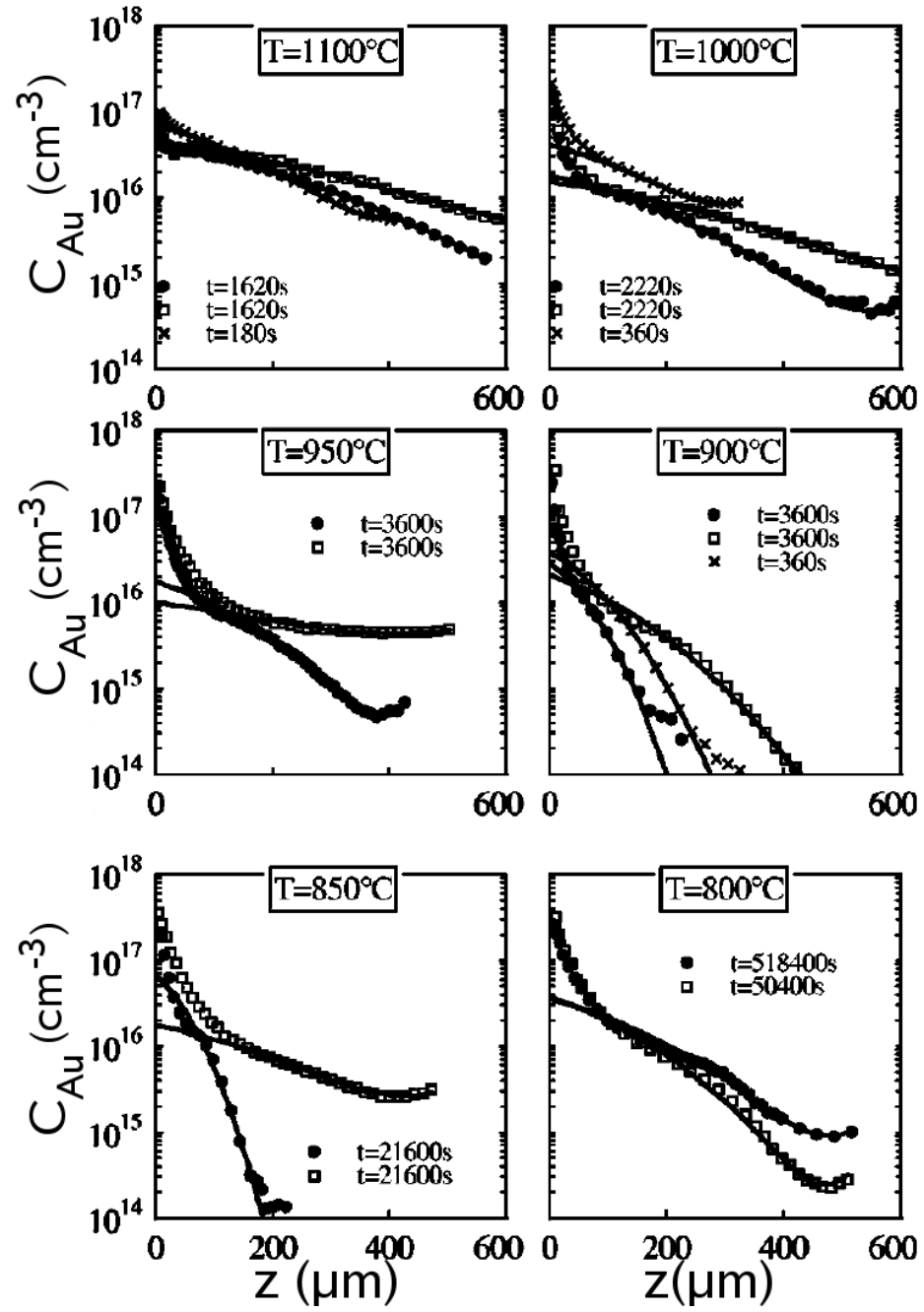

Abbildung 5.6 - NAA-Messungen von Goldkonzentrationsprofilen in versetzungshaltigen Silizium (aus $\underline{[20]}$ ). Die unterschiedlichen Profile resultieren aus unterschiedlichen Diffusionszeiten und -temperaturen sowie aus unterschiedlichen Dotierkonzentrationen (Kreis: praktisch undotiert, Quadrat: $[\mathrm{B}]=3 \cdot 10^{19} \mathrm{~cm}^{-3}$, Kreuz: $[\mathrm{B}]=1.7 \cdot 10^{20} \mathrm{~cm}^{-3}$ ). Die durchgezogenen Linien sind die Ergebnisse von Anpassungen einer Fehlerfunktion.

eingezeichneten durchgezogenen Linien stellen das Ergebnis einer Anpassung dieser Pro- 
file mit der Gleichung

$$
C_{\mathrm{Au}}(z)=C_{\mathrm{Au}}^{\mathrm{eq}}=\left(\operatorname{erfc}\left(\frac{z}{2 \sqrt{D_{\mathrm{Au}}^{\mathrm{T}, *} t}}\right)+\operatorname{erfc}\left(\frac{d-z}{2 \sqrt{D_{\mathrm{Au}}^{\mathrm{T}, *} t}}\right)\right)
$$

dar. Hier sind $C_{\mathrm{Au}}$ die Gesamtkonzentration des Goldes, $d$ die Probendicke, $z$ der Abstand zur Oberfläche und $t$ die Diffusionszeit. $C_{\mathrm{Au}}^{\mathrm{eq}}$ und $D_{\mathrm{Au}}^{\mathrm{T}, *}$ als Löslichkeit und effektiver Diffusionskoeffizient stellen die freien Parameter der Anpassung dar. Diese Gleichung entspricht der Gl. (2.24), wobei in der Anpassung die Diffusion von den beiden gegenüberliegenden Probenflächen berücksichtigt werden muss. Die in Oberflächennähe von den berechneten Profilen abweichenden Messwerte wurden mit der Existenz von Goldteilchen begründet, die mit TEM in diesen Bereichen beobachtet wurden (vgl. auch Kap. 4.3). Diese Bereiche wurden bei der Anpassung nicht berücksichtigt. Durch Extrapolation der berechneten Profile auf $\mathrm{z}=0$ wurden Randkonzentrationen bestimmt und als Löslichkeit von Gold in versetzungshaltigem Silizium interpretiert. Diese sind in der Abb. 5.8(a) für Ergebnisse der undotierten Proben eingetragen. Dieser Extrapolation liegt die Annahme zugrunde, dass die Gleichgewichtskonzentration durch die flüssige Phase auf der Oberfläche eingestellt wird (vgl. Kap. 2.2.2). Der Anpassung der gemessenen Konzentrationsprofile mit einer Fehlerfunktion gemäß Gl. (5.5) liegt die Annahme eines konzentrationsunabhängigen Diffusionskoeffizienten zugrunde. Grundlage der Herleitung des in Kap. 2.2.1 beschrieben effektiven Diffusionskoeffizienten $D_{\mathrm{Au}}^{\mathrm{T}, *}(\mathrm{Gl} .(2.26)$ ) ist die Gleichung

$$
D_{\mathrm{Au}}^{\mathrm{T}, *}=\frac{C_{\mathrm{i}}^{\mathrm{eq}} D_{\mathrm{i}}}{C_{\mathrm{i}}^{\mathrm{eq}}+C_{\mathrm{s}}^{\mathrm{eq}}+f_{\mathrm{T}} C_{\mathrm{Au}}^{\mathrm{eq}}} .
$$

Die $C^{\text {eq }}$ stellen die Gleichgewichtskonzentrationen der interstitiellen und substitutionellen Goldatome (Index i, s) sowie die Gleichgewichtskonzentration der im Versetzungskern gebundenen Goldatome (Index $\mathrm{Au}_{\mathrm{T}}$ ) dar. $D_{\mathrm{i}}$ bezeichnet den Diffusionskoeffizienten der interstitiellen Goldatome. $f_{\mathrm{T}}$ lässt sich folgendermaßen darstellen:

$$
f_{\mathrm{T}}=\frac{C_{\mathrm{T}}\left(C_{\mathrm{T}}-C_{d}^{\mathrm{eq}}\right)}{\left(C_{\mathrm{T}}+C_{\mathrm{d}}^{\mathrm{eq}}\left(\frac{C_{\mathrm{s}}}{C_{\mathrm{s}}^{\mathrm{eq}}}-1\right)\right)^{2}}
$$

$C_{\mathrm{s}}$ als Konzentration der substitutionellen Goldatome ist eine ortsabhängige Größe. Hier bezeichnen $C_{0}^{\mathrm{eq}}$ und $C_{\mathrm{T}}$ die Konzentrationen der leeren bzw. der gesamten Bindungsplätze an den Versetzungen sowie $C_{\mathrm{d}}^{\text {eq }}$ die Löslichkeiten der dort gebundenen Goldatome. Für den bei Rodriguez und Mitarbeitern angenommenen Fall eines verschwindenden Besetzungsgrades für Goldatome an den Versetzungen $\left(C_{\mathrm{d}}^{\mathrm{eq}} \ll C_{\mathrm{T}} \approx C_{0}^{\mathrm{eq}}\right.$, vgl. Kap. 2.2.1) wird $f_{\mathrm{T}}=1$ und $D_{\mathrm{Au}}^{\mathrm{T}, *}$ unabhängig vom Ort.

Die Bestimmung einer Bindungsenergie von Goldatomen an den Versetzungen erfolgte durch Anpassung eines Segregationskoeffizienten, der unter anderem durch das Verhält- 
nis aus dem Kristallvolumen und dem Gesamtvolumen der Versetzungen gegeben ist (Gl. (2.27)) . Hier wird der Radius einer Versetzung mit der Länge einer halben Gitterkonstante $(0.27 \mathrm{~nm})$ und die Versetzungsdichte mit $10^{8} \mathrm{~cm}^{-2}$ angegeben. Die Herleitung dieses Segregationskoeffizienten wird in Kap. 2.2.1 skizziert.

Der folgende Abschnitt bewertet die in dieser Interpretation gemachten Annahmen und liefert einen Vorschlag für eine andere Deutung mit dem Resultat der Abschätzung einer Bindungsenergie.

\subsubsection{Alternative Interpretation der Ergebnisse einer anderen Veröffentlichung}

Die Betrachtungen über Gleichgewichtskonzentrationen in Anwesenheit von Versetzungen zu Beginn dieses Unterkapitels treffen auch auf die Arbeit von RoDRIGUEz UND Mitarbeitern zu. Die dort wie in dieser Arbeit gefundenen Goldausscheidungen in der Nähe der Golddiffusionsquelle (vgl. Kap. 4.3), die unter vermeintlichen Gleichgewichtsbedingungen während der Eindiffusion entstanden sind, stützen diese Betrachtungen. Bei deren Entstehung handelt es sich vermutlich um das Resultat transienter Prozesse, die mit dem Abbau von Nichtgleichgewichten intrinsischer, bei der Verformung entstandener Punktdefekte in Verbindung stehen. Die Existenz dieser Ausscheidungen wird in ${ }^{[20]}$ als Begründung für den konvexen Verlauf der Konzentrationsprofile innerhalb des Bereichs bis $100 \mu \mathrm{m}$ genannt. Folgerichtig sollte in diesen Bereichen in etwa die Gleichgewichtskonzentrationen von interstitiellem und substitutionellem Gold bezüglich der Ausscheidungsphase eingestellt sein, und - sofern lokales Gleichgewicht angenommen werden kann - auch die der mit den goldinduzierten Akzeptorzuständen assoziierte Goldspezies im Versetzungskern. Das bedeutet wiederum, dass die Ausscheidungsbildung in das Diffusionsmodell integriert werden muss, was in der Behandlung von RodRIGUEz UND MitarbeITERN nicht geschehen ist. In einer ersten Näherung kann angenommen

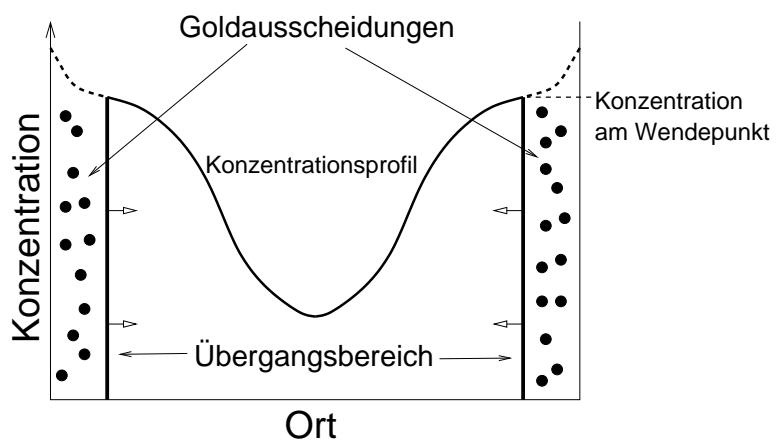

Abbildung 5.7 - Schema eines Konzentrationsprofils mit zeitabhängiger Randbedingung. Der Übergangsbereich bewegt sich während der Eindiffusion weiter in die Probe, dahinter entstehen Ausscheidungen. 
werden, dass die Ausscheidungen die Gleichgewichtskonzentrationen des interstitiellen und substitutionellen Goldes einstellen. Die Daten aus ${ }^{[20]}$ legen ferner nahe, dass sich der ausscheidungsbehaftete Bereich während der Eindiffusion in die Probe hinschiebt, also näherungsweise Eindiffusion aus einer bewegten Diffusionsquelle stattfindet. Diesen Zusammenhang verdeutlicht die Abb. 5.7. Dies widerspricht der Annahme, dass die Gesamtlöslichkeit des Goldes durch Extrapolation der angepassten Fehlerfunktionen auf die Oberfläche zu ermitteln ist. Eine erste Annahme, die in dieser Arbeit getroffen wird, ist demzufolge, dass sich dass Gleichgewicht dort einstellt, wo die Konzentrationsprofile ihre Krümmung von konvex nach konkav ändern. Die zu diesen Bereichen gehörenden Konzentrationen sind in der Abb. 5.8 unter der Bezeichnung „Wendepunkt" eingetragen.

Die Berechnung eines Segregationskoeffizienten auf der Grundlage des Gesamtvolumens der Versetzungen wie sie oben beschrieben ist, legt die Annahme zugrunde, dass die Löslichkeit des Goldes an den Versetzungen von deren Volumen abhängt. Bei einer angenommenen Versetzungsdichte von $10^{8} \mathrm{~cm}^{-2}$ und einer minimalen gemessenen Löslichkeit von $10^{16} \mathrm{~cm}^{-3}$ müsste die Dichte von Goldatomen entlang der Versetzungen $10^{8} \mathrm{~cm}^{-1}$ betragen (Die Dichte der substitutionellen Goldatome liegt bei $800^{\circ} \mathrm{C}$ bei ca. $5 \cdot 10^{14} \mathrm{~cm}^{-3}$ und kann daher vernachlässigt werden). Dann müssten im Versetzungskern durchschnittlich ca. 3.8 Atome auf einer Burgersvektorlänge $(3.84 \mathrm{~nm})$ gebunden werden. Diese Burgersvektorlänge ist der Abstand zweier benachbarter Atome im Kern einer Partialversetzung (vgl. Kap. 2.3.1.1). Bei zwei Partialversetzungen lassen sich aber maximal zwei Atome auf dieser Länge binden. Somit führt diese Abschätzung zu einem Widerspruch zu der oben gemachten Annahme, zumal dort bei der Herleitung des effektiven Diffusionskoeffizienten (Gl. (5.6) , (5.7)) von einem kleinen Besetzungsgrad ausgegangen wird.

Eine zweite Annahme zur Neuinterpretation der Ergebnisse von RoDRIGUEz UND MITARBEITERN ist, dass pro Burgersvektorlänge eine mögliche Haftstelle für Goldatome zur Verfügung steht. Im folgenden soll auf der Grundlage einer chemischen Reaktion ähnlich der in Kap. 2.2.1 beschriebenen ,kick-out"-Reaktion (Gl. (2.21)) ein Besetzungsgrad dieser Bindungsplätze hergeleitet werden.

Dabei reagiert ein substitutionelles Goldatom $\mathrm{Au}_{\mathrm{s}}$ mit einer leeren Haftstelle im Versetzungskern $\mathrm{T}_{0}$,

$$
\mathrm{Au}_{\mathrm{s}}+\mathrm{T}_{0} \rightleftharpoons \mathrm{Au}_{\mathrm{d}}
$$

$\mathrm{zu}$ einem im Versetzungskern gebundenen Goldatom $\mathrm{Au}_{\mathrm{d}}$. Mit dem Massenwirkungsgesetz gilt im thermodynamischen Gleichgewicht

$$
\begin{aligned}
C_{\mathrm{s}}^{\mathrm{eq}} \cdot C_{0}^{\mathrm{eq}} & =C_{\mathrm{d}}^{\mathrm{eq}} C_{\mathrm{Si}} \cdot \exp \left(-\frac{\Delta G_{\mathrm{Au}_{\mathrm{d}}}}{k_{\mathrm{B}} T}\right) \text { mit } \\
C_{0}^{\mathrm{eq}} & =C_{\mathrm{T}}^{\mathrm{eq}}-C_{\mathrm{d}} .
\end{aligned}
$$

$C_{\mathrm{Si}}$ stellt die Konzentration der im Siliziumgitter zur Verfügung stehenden Gitterplätze 


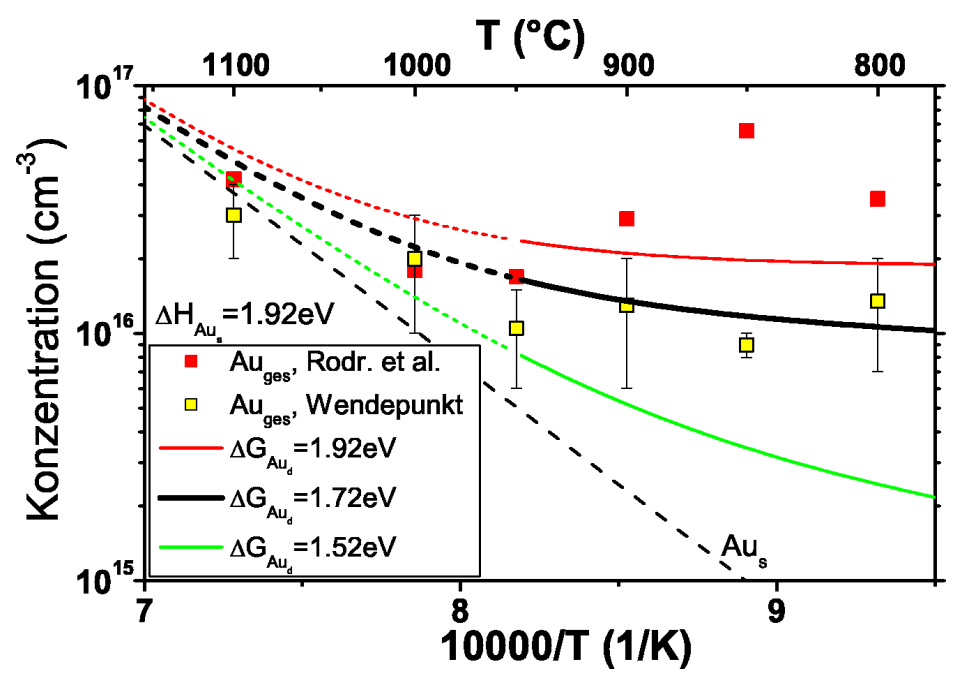

(a) Variation der Bindungsenergien

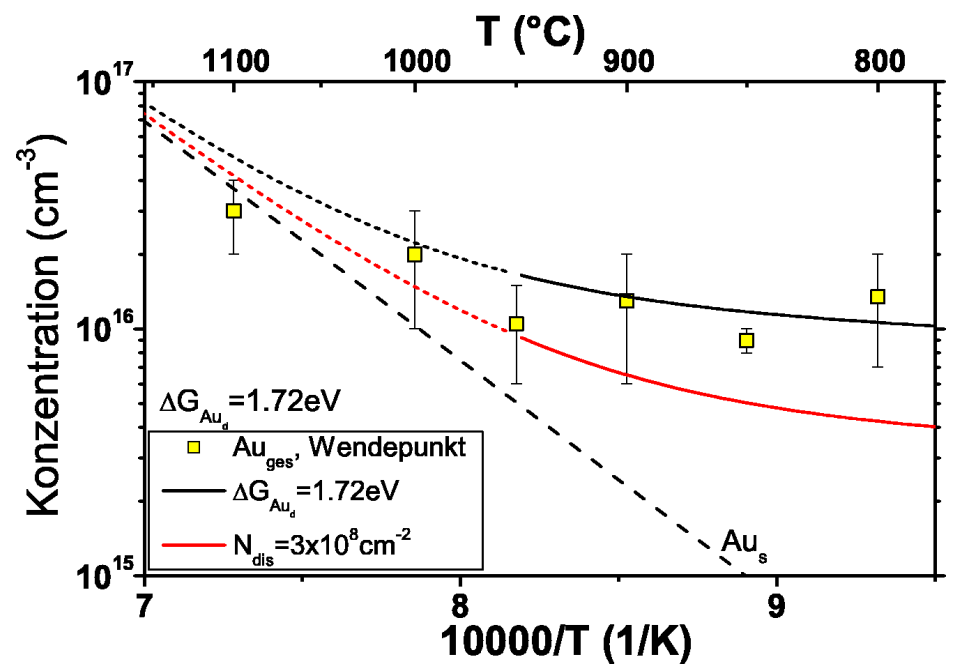

(b) Variation der Versetzungsdichten

Abbildung 5.8 - a) Auftragung der durch Extrapolation der Konzentrationsprofile gewonnenen Löslichkeiten (rote Quadrate) sowie der Löslichkeiten, die an der Änderung der Krümmung abgelesen wurden (gelbe Quadrate). Die Fehlerbalken geben den Konzentrationsbereich an, der auf die unterschiedlichen experimentellen Bedingungen zurückzuführen ist. Die gestrichelte Linie zeigt das Ergebnis der Berechnung für die Löslichkeit substitutioneller Goldatome, die durchgezogenen und für höhere Temperaturen gestrichelten Linien stellen die für unterschiedliche Bindungsenergien gemäß Gl. (5.13) berechnete Gesamtlöslichkeit dar. b) Hier zeigen die durchgezogenen (teilweise gestrichelten) Linien die zu erwartende Löslichkeit bei einer Bindungsenergie von $1.72 \mathrm{eV}$ für unterschiedliche Versetzungsdichten. 
dar. $\Delta G_{\mathrm{Au}_{\mathrm{d}}}$ ist die Differenz der freien Enthalpie eines Goldatomes auf einem substitutionellem Gitterplatz und der eines Goldatomes im Versetzungskern (Bindungsenergie). Daraus berechnet sich die Wahrscheinlichkeit der Besetzung einer Haftstelle mit einem Goldatom $f_{\mathrm{Au}_{\mathrm{d}}} \mathrm{zu}$

$$
f_{\mathrm{Au}_{\mathrm{d}}}=\frac{C_{\mathrm{d}}^{\mathrm{eq}}}{C_{\mathrm{T}}}=\frac{1}{1+\frac{C_{\mathrm{Si}}}{C_{\mathrm{s}}^{\mathrm{eq}}} \cdot \exp \left(-\frac{\Delta G_{\mathrm{Au}_{\mathrm{d}}}}{k_{\mathrm{B}} T}\right)} .
$$

Die Löslichkeit $C_{\mathrm{Au}}^{\mathrm{eq}}$ unter der Berücksichtigung der Segregation im Versetzungskern ist dann

$$
\begin{aligned}
C_{\mathrm{Au}}^{\mathrm{eq}} & =C_{\mathrm{i}}^{\mathrm{eq}}+C_{\mathrm{s}}^{\mathrm{eq}}+f_{\mathrm{Au}_{\mathrm{d}}} \cdot N_{\mathrm{L}} \cdot N_{\mathrm{d}} \\
& \approx C_{\mathrm{s}}^{\mathrm{eq}}+f_{\mathrm{Au}_{\mathrm{d}}} \cdot N_{\mathrm{L}} \cdot N_{\mathrm{d}}
\end{aligned}
$$

wobei $C_{\mathrm{i}}^{\mathrm{eq}}$ und $C_{\mathrm{s}}^{\mathrm{eq}}$ die Löslichkeit der interstitiellen und der substitutionellen Goldatome bedeuten. $N_{\mathrm{L}}$ und $N_{\mathrm{d}}$ beschreiben die Liniendichte der Bindungsplätze entlang der Versetzung bzw. die Versetzungsdichte. In Abb. 5.8(a) ist $C_{\mathrm{s}}^{\mathrm{eq}}$ durch eine gestrichelte Linie dargestellt. Die Berechnung erfolgte auf Grundlage der Daten aus ${ }^{[80]}, \Delta H_{\mathrm{Au}_{\mathrm{s}}}=1.92 \mathrm{eV}$ und $\Delta S_{\mathrm{Au}_{\mathrm{s}}}=2.8 \cdot k_{\mathrm{B}}$, sowie der Gleichung

$$
C_{\mathrm{s}}^{\mathrm{eq}} \approx 5 \cdot 10^{22} \mathrm{~cm}^{-3} \cdot 0.5 \cdot \exp \left(-\frac{\Delta H_{\mathrm{Au}_{\mathrm{s}}}+\Delta S_{\mathrm{Au}_{\mathrm{s}}} \cdot T}{k_{\mathrm{B}} T}\right)
$$

In der flüssigen Phase, mit der sich die Konzentration im Silizium im Gleichgewicht befindet, entspricht das Verhältnis von Siliziumatomen und Goldatomen dem Faktor 0.5 bei $940^{\circ} \mathrm{C}$. In dem in Abb. 5.8 aufgetragenen Temperaturbereich weicht dieser Faktor davon maximal um etwa $10 \%$ ab (siehe Phasendiagramm im Anhang C). Daher wird dieser Wert hier konstant gehalten. Als Dichte der möglichen Bindungsplätze entlang der Versetzungslinien $N_{\mathrm{L}}$ wurde, wie oben erklärt, der Kehrwert der Länge eines Burgersvektors $b^{-1}=3.84 \mathrm{~nm}^{-1}$ gewählt. Mit diesen Annahmen folgen die der Abb. 5.8 gezeigten Verläufe, die dort mit den Werten aus den Diffusionsprofilen (Abb. 5.6) verglichen werden. Die durchgezogenen, teilweise gestrichelten Linien in Abb. 5.8(a) wurden mit Gl. (5.13) und unterschiedlichen Bindungsenergien $\Delta G_{\mathrm{Au}_{\mathrm{d}}}$ und der in Kap. 4.1.3 für eine Probe dieser Experimente angegebenen Versetzungsdichte von $8 \cdot 10^{8} \mathrm{~cm}^{-2}$ gerechnet. Die Versetzungsdichte wurde als eine Grundlage dieser Analyse durch Auswertung von STEM-Aufnahmen ermittelt (vgl. Kap.4.1.3). Um zu zeigen, dass diese Linien im oberen Temperaturbereich lediglich eine Extrapolation darstellen, weil mit Ausheilungsprozessen der Versetzungen und daher mit einer Reduktion der Versetzungsdichte zu rechnen ist, wurde dieser Bereich der Linien gestrichelt dargestellt. In Kap. 4.1 .2 wird gezeigt, dass für die hier verwendeten Proben und den Diffusionstemperaturen und -zeiten, wie sie in 4.1.2 angewendet wurden, mit einer auffälligen Reduktion der Versetzungsdichte spätestens ab $1100^{\circ} \mathrm{C}$ zu rechnen ist. Jedoch unterscheiden sich diese Proben und die in 
4.1.2 durch ihre Verformungsbedingungen und Ausgangsversetzungsdichten.

In dieser Interpretation der von RODRIGUEZ Und MITARBEITERN erzielten Ergebnisse beträgt die Bindungsenergie von Goldatomen am Versetzungskern ca. $\Delta G_{\mathrm{Au}_{\mathrm{d}}} \approx 1.7 \pm 0.2 \mathrm{eV}$. Wie aus der Abb. 5.8(a) hervorgeht, befinden sich zwischen den Verläufen, die mit $1.7 \pm 0.2 \mathrm{eV}$ gerechnet wurden, alle aus den Diffusionsprofilen abgelesenen „Wendepunkte" für Temperaturen unterhalb $1000^{\circ} \mathrm{C}$. Diese Bindungsenergie ist in guter Übereinstimmung mit den von FuJITA UND MITARBEITERN ${ }^{[15]}$ berechneten Werte für einzelne Goldatome (vgl. Kap. 2.4.1). In der Abb. 5.8(b) sind die durchgezogenen, teilweise gestrichelten Linien mit einer Bindungsenergie von $1.72 \mathrm{eV}$ und zwei unterschiedlichen Versetzungsdichten gerechnet worden. Die Abweichungen der Konzentrationen von der schwarzen Linie, die mit der Versetzungsdichte von $8 \cdot 10^{8} \mathrm{~cm}^{-2}$ berechnet wurde, für Temperaturen oberhalb von $900^{\circ} \mathrm{C}$ lassen sich vermutlich durch eine Abnahme der Versetzungsdichten aufgrund der Temperaturbehandlung erklären, die sich während der Eindiffusion ergibt. In Kap. 4.1.2 konnte anhand einer Probe mit der Versetzungsdichte von $3.3 \cdot 10^{8} \mathrm{~cm}^{-2}$ gezeigt werden, dass diese durch Auslagerung bei $1100^{\circ} \mathrm{C}$ für $27 \mathrm{~min}$ auf ca. $1 / 4$ ihres ursprünglichen Wertes reduziert wird.

Wenn in der gleichen Weise mit dem anderen, in ${ }^{[80]}$ angegebenen Wertepaar für $\Delta H_{\mathrm{Au}_{\mathrm{s}}}=1.98 \mathrm{eV}$ und $\Delta S_{\mathrm{Au}_{\mathrm{s}}}=3.8 \cdot k_{\mathrm{B}}$ eine Bindungsenergie bestimmt wird, so wird ebenfalls ein Wert von $1.7 \mathrm{eV}$ für die Bindungsenergie ermittelt. Dann gibt es allerdings im Temperaturbereich oberhalb von $900^{\circ} \mathrm{C}$ deutliche Abweichungen. Mit der Bindungsenergie von $1.7 \mathrm{eV}$ berechnet sich die Besetzung der Bindungsplätze im Versetzungskern mit Goldatomen bei $800^{\circ} \mathrm{C}$ zu $f_{\mathrm{Au}_{\mathrm{d}}} \approx 0.4$ bis 0.5 , abhängig von den für $\Delta H_{\mathrm{Au}_{\mathrm{s}}}$ und $\Delta S_{\mathrm{Au}_{\mathrm{s}}}$ gewählten Werten.

Eine andere Annahme ist, dass sowohl die $30^{\circ}$ - als auch die $90^{\circ}$-Partialversetzung in gleicher Weise Goldatome im Versetzungskern binden können. Für diesen Fall muss die Bindungsplatzdichte auf zwei pro Burgersvektorlänge erhöht werden. Dann verringert sich die auf diese Weise berechnete Bindungsenergie mit beiden Datensätzen auf ca. $1.6 \mathrm{eV}$, was innerhalb der oben angenommenen Fehlergrenze $1.7 \pm 0.2 \mathrm{eV}$ liegt. Dann jedoch liegt der Besetzungsgrad bei $f_{\mathrm{Au}_{\mathrm{d}}} \approx 0.2$ bis 0.5 .

In beiden Fällen widerspricht dies der Annahme einer kleinen Besetzung, wie sie RoDRIGUEZ Und Mitarbeiter treffen (vgl. Kap. 5.3.1). Diese Annahme wird benötigt, um einen ortsunabhängigen effektiven Diffusionskoeffizienten zu begründen. Die Anpassung der Konzentrationsprofile durch einer erfc-Funktion (Gl. (5.5)), die einen ortsunabhängigen Diffusionskoeffizienten voraussetzt ${ }^{[81]}$, gelingt möglicherweise aus folgendem Grund: In den Bereichen zwischen den Übergangsbereichen (Abb. 5.7) ist die Konzentration substitutioneller Goldatome noch weit weg vom Gleichgewicht $\left(\frac{C_{\mathrm{s}}}{C_{\mathrm{s}}^{\mathrm{qg}}} \ll 1\right)$. In diesem Fall wird $f_{\mathrm{T}}$ aus Gl. (5.7) und damit auch der Diffusionskoeffizient $D_{\mathrm{Au}}^{\mathrm{T}, *}$ (Gl. (5.6) ) ebenfalls unabhängig vom Ort. 


\subsection{Einfluss der Versetzungen auf die Golddiffusion}

Die in dieser Arbeit erzielten Ergebnisse reichen nicht aus, um die Golddiffusion in versetzungshaltigem Silizium genauer zu analysieren. Ein wichtiger Grund dafür ist, dass mit der hier verwendeten elektrischen Messmethode die Gesamtkonzentration des Goldes nicht zugänglich ist. Allerdings fällt an den Messungen dieser Arbeit auf, dass die Diffusion offensichtlich stark von der Versetzungsdichte abhängt, die sich bei den beiden ndotierten Proben lediglich um etwa einen Faktor 2.5 unterscheidet. In der Abb. 4.14wird dies insbesondere anhand der Messungen an der Probe mit der geringeren Versetzungsdichte deutlich. Hier kann auch in einer Tiefe von $317 \mu \mathrm{m}$ und $328 \mu \mathrm{m}$ ein deutliches Signal des Akzeptorzustandes substitutionell gelösten Goldes gemessen werden, deren Einfangkinetik sich nicht von dem in den versetzungsfreien Proben unterscheidet. Offensichtlich stand der Ladungsträgereinfang hier nicht unter dem Einfluss eines elektrostatischen Potentials (Dies erklärt die zunächst unerwartet hoch erscheinenden Linienamplituden, da dieser Zustand nur hier vollständig umgeladen werden konnte). Daher ist anzunehmen, dass die Goldkonzentration an den Versetzungen und damit das elektrostatische Potential hier zu gering war, um die Einfangkinetiken messbar zu beeinflussen. In der Probe mit der höheren Versetzungsdichte fällt die Amplitude der substitutionell gelösten Goldatome wie in der p-dotierten Probe mit der gleichen Versetzungsdichte schon bei einem Abstand von ca. $150 \mu$ auf ein nicht mehr messbares Maß ab.

Bei der Bewertung der Unterschiede in den Eindringtiefen des substitutionellen Goldes ist zu berücksichtigen, dass die Dichte der Bindungsplätze für Gold an den Versetzungen ebenfalls um den Faktor 2.5 variiert. Dabei sind die Bindungsplätze auf der Längenskala der Versetzungsabstände nicht homogen verteilt. Es ist anzunehmen, dass der Einbau von Goldatomen somit nicht nur von der mittleren Dichte der Bindungsplätze sondern auch von deren Verteilung abhängt, d.h. bei größerer lokaler Dichte der Bindungsplätze verzögert sich der Einbau mit zunehmender Konzentration. Damit verzögert sich auch die Einstellung lokaler Gleichgewichte in Proben mit größerer Versetzungsdichte. Darüber hinaus lässt sich aus der im Vergleich zu versetzungsfreien Proben unveränderte Einfangkinetik der Goldakzeptorzustände in großen Tiefen $(>317 \mu \mathrm{m})$ nicht geschlossen werden, dass hier kein Gold an den Versetzungen vorliegt. Eine Verringerung der Liniendichte der Goldatome entlang der Versetzungen wirkt sich in zweifacher Weise auf die in der DLTS gemessene Einfangkinetik aus: Zum einen verringert sich dadurch das Potential, was die mittlere Einfangrate der im Read-Radius befindlichen Goldakzeptorzustände erhöht. Zum anderen wird der Anteil der im Read-Radius befindlichen Zustände verringert. Somit kann dieser Effekt nur quantitativ analysiert werden. 


\subsection{Auffälligkeit in den DLTS-Spektren der versetzungshaltigen $\mathrm{p}$-dotierten Probe}

In diesem Abschnitt soll kurz auf den in Abb. 4.25 gezeigten Effekt eingegangen werden. Diese Abbildung ist in größerer Auflösung noch einmal in Abb. 5.9 dargestellt.

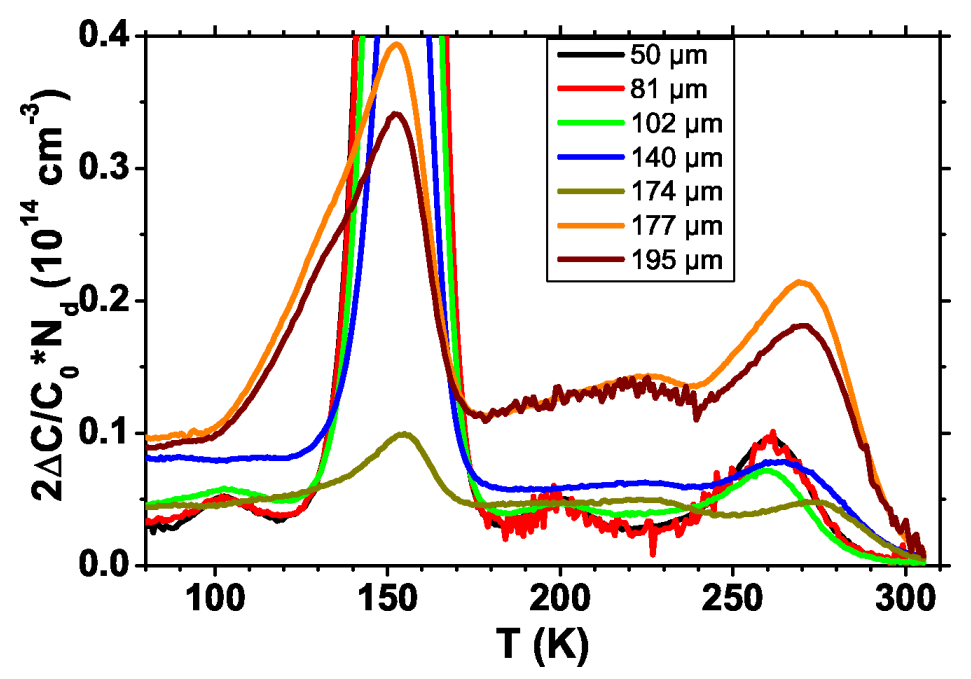

Abbildung 5.9 - Vergleich einiger Spektren der p-dotierten versetzungshaltigen Probe. Die Emission, die zur asymmetrischen Verbreiterung führt, tritt erst in Erscheinung, wenn die Amplitude der Golddonatorzustände deutlich reduziert ist.

Hieraus geht eine Abnahme der Konzentration substitutionellen Goldes hervor, der etwa im gleichen Abstand zur Diffusionsquelle auftritt, wie das Erscheinen der versetzungsinduzierten Linien.

Es konnte gezeigt werden, dass die flüssige Phase der Goldschicht auf der Oberflä-

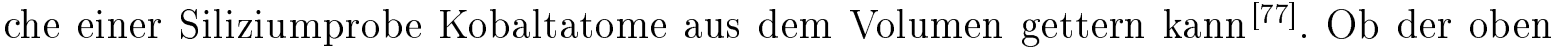
beschriebene Effekt auf das Gettern von Verunreinigungen zurückzuführen ist, die die beschriebenen DLTS-Signale in Verbindung mit Versetzungen hervorbringen, kann nur vermutet werden. Damit kann jedoch nicht erklärt werden, warum die Abnahme der Goldkonzentration und die Zunahme der Konzentration versetzungsinduzierter Defekte an der gleichen Stelle stattfindet. Für eine Klärung dieses Zusammenhangs sind daher weitere Experimente nötig. 


\section{Zusammenfassung und Ausblick}

In der vorliegenden Arbeit wurden Wechselwirkungen von Gold und Versetzungen in Silizium mittels der Kapazitätstransientenspektroskopie (DLTS, „Deep Level Transient Spectroscopy") untersucht. Die Proben unterschieden sich in der Art der Dotierung (pund n-Dotierung) und in der Versetzungsdichte. Darüber hinaus konnte durch den Einsatz einer speziellen Präparationstechnik der Einfluss variierender Goldkonzentrationen bestimmt werden. Neben den DLTS-Messungen brachten Messungen der temperaturabhängigen Dichten freier Ladungsträger wichtige Erkenntnisse.

Zunächst wurden die elektrischen Eigenschaften von Gold in versetzungsfreiem Silizium mittels DLTS bestimmt. Neben dem Akzeptor- und dem Donatorzustand des substitutionell gelösten Goldes konnten weitere, dem Gold-Wasserstoff-Komplex zugeordnete tiefe Zustände nachgewiesen werden. Mithilfe einer Methode zur numerischen Anpassung der DLTS-Spektren wurden Eigenschaften dieser tiefen Zustände ermittelt. Dies waren die Lage des Zustandes in der Bandlücke, der Einfangquerschnitt und der Entropiefaktor der Umladung des Zustandes. Dabei wurde einerseits ausgenutzt, dass die Akzeptorzustände des substitutionell gelösten Goldes und des Gold-Wasserstoff-Komplexes aufgrund deren Lage in der Mitte der Bandlücke sowohl im Spektrum der n-dotierten als auch in dem der p-dotierten Probe nachgewiesen werden können. Andererseits kam dieser Analyse zugute, dass der Gold-Wasserstoff-Komplex drei Zustände in die Bandlücke des Siliziums einführt, wobei jeweils zwei mit gleicher Konzentration im DLTS-Spektrum beider Dotierungen Linien erzeugen. Die auf diese Weise ermittelten Werte zeigten gute Übereinstimmungen mit den Daten aus der Literatur. Außerdem konnte bestätigt werden, dass der Gold-Wasserstoff-Komplex durch Auslagerungsexperimente dissoziiert werden kann. Für p-dotiertes Silizium wurde gezeigt, dass unterschiedliche Kontaktpräparationsschritte zu unterschiedlichen Konzentrationen des Gold-Wasserstoff-Komplexes führte.

Versetzungen, die nicht mit metallischen Fremdatomen dekoriert sind, zeigen nur eine sehr schwache Rekombinationsaktivität. Theoretische Untersuchen haben gezeigt, dass Versetzungen aufgrund fast vollständig rekonstruierter Versetzungskerne keine tiefen Zustände in die Bandlücke des Siliziums einführen. Unter anderem deswegen werden die Linien, die im DLTS-Spektrum versetzungshaltiger Siliziumproben gemessen werden, metallischen Verunreinigungen an den Versetzungen zugeordnet. Diese Linien konnten auch in den golddotierten Proben dieser Arbeit nachgewiesen werden. Darüber hinaus wurden wiederum die Linien des substitutionellen Goldes gemessen.

Die genaue Kenntnis der Eigenschaften von Gold in versetzungsfreiem Silizium ermög- 
lichte eine konsistente Zuordnung von Effekten, die in den versetzungshaltigen Proben auftraten. Neben den gerade erwähnten, versetzungsinduzierten Linien gehörten dazu insbesondere eine veränderte Einfangkinetik des Goldakzeptorzustandes, deutlich abweichende Dichten freier Ladungsträger und versetzungsdichteabhängige Diffusionsprofile, die qualitativ aus den DLTS-Linienamplituden der Zustände substitutioneller Goldatome abgeleitet werden konnten. Außerdem stellte sich heraus, dass Versetzungen die Bildung von Gold-Wasserstoff-Komplexen hemmt, was möglicherweise auf konkurrierende Reaktionen des Wasserstoffs mit gold- und versetzungskorrelierten Defekten zurückzuführen ist.

Zunächst wurde festgestellt, dass die freien Ladungsträgerdichten in den n-dotierten Proben zum Teil deutlich reduziert waren. Dieser Effekt war stärker in der Probe mit der höheren Versetzungsdichte. Die Abnahme war zudem mit der Konzentration der substitutionellen Goldatome gekoppelt. Andererseits wurde ein solches Verhalten in der p-dotierten Probe nicht nachgewiesen. Aus diesen Ergebnissen wurde auf einen tiefen Akzeptorzustand geschlossen, der durch Goldatome im Versetzungskern in die Bandlücke eingeführt wird. Dass Goldatome mit einer beträchtlichen Liniendichte im Versetzungskern gebunden sind, wurde durch Experimente von RoDRIGUEz UND MitARBEITERN [20] nachgewiesen, deren Ergebnisse einer neuen Analyse unterzogen wurden. Das Ergebnis dieser Analyse war eine Bindungsenergie von $1.7 \pm 0.2 \mathrm{eV}$ für Goldatome im Versetzungskern bezogen auf die substitutionelle Spezies im ungestörten Kristall. Eine derartige große Bindungsenergie korrespondiert mit einem Besetzungsgrad im Versetzungskern von nahezu $50 \%$ bei der hier angewendeten Diffusionstemperatur von $800^{\circ} \mathrm{C}$.

Der geladene Akzeptorzustand der Goldatome entlang der Versetzungen ist verbunden mit einer Linienladung, die durch die ionisierten Dotieratome in der Umgebung abgeschirmt wird. Das hiermit verknüpfte elektrostatische Potential führt in einem zylindersymmetrischen Gebiet um die Versetzungen zu einer Reduktion der Dichte freier Elektronen. Als Folge tritt für tiefe Zustände im Einflussbereich dieses Potentials eine verlangsamte Einfangkinetik auf. In der DLTS macht sich dieses durch eine veränderte Abhängigkeit der Linienamplitude von der Einfangdauer bemerkbar. Damit dieser Effekt gemessen werden kann, muss ein beträchtlicher Anteil der entsprechenden Atome innerhalb des besagten zylindersymmetrischen Potentials vorliegen, da alle außerhalb befindlichen Atome mit einer ungestörten Einfangkinetik zum gemessenen Mittelwert beitragen. Zum anderen darf der Einfangquerschnitt des betreffenden Defektes einen gewissen Wert nicht überschreiten. Beide Anforderungen erfüllte der Akzeptorzustand des substitutionell gelösten Goldes, der, wie oben beschrieben, eine abweichende Einfangkinetik aufwies. Somit führte das Bild eines tiefen Akzeptorzustandes der Goldatome im Versetzungskern zu einem konsistenten Bild. Dieser Fall einer großen Dichte tiefer Zustände innerhalb des Potentials erschwert dessen mathematische Beschreibung. Ein Modell für ein zylindersymmetrisches Potential, dessen abschirmende Ladungsdichte zeitabhängig ist, liegt bislang nicht vor. Die Entwicklung eines solchen Modells müsste nach Vorbild des Modells von Pons ${ }^{[23]}$ erfolgen. Dies ist eine selbst-konsistente Beschreibung 
der Einfangkinetik von Zuständen mit großer Dichte im Potential der Raumladungszone eines Schottky-Kontaktes.

Der tiefe goldinduzierte Akzeptorzustand an den Versetzungen konnte weder in den Spektren der p-dotierten noch in denen der n-dotierten Proben nachgewiesen werden. Zur Bestimmung der Lage des Energieniveaus wurden zwei Modelle diskutiert, die jeweils Grenzfälle der Beschreibung von Linienladungen entlang der Versetzungen darstellen. Diese Grenzfälle bestehen zum einen in der Annahme vollständig lokalisierter Zustände [48] und zum anderen in der Annahme vollständig delokalisierter, d.h bandartiger Zustände entlang der Versetzungen ${ }^{[78]}$. Daraus folgte die begründete Hypothese, dass es sich bei den tiefen goldinduzierten Versetzungszuständen um bandartige Zustände handeln müsste, deren Zustand in der unteren Hälfte der Bandlücke anzusiedeln ist. Von einem bandartigen Zustand mit einer Zustandsdichte, die sich über ein Energieintervall von einigen $100 \mathrm{meV}$ erstreckt, kann erwartet werden, dass dieser kein auffälliges Signal im DLTS-Spektrum bewirkt. Hall-Effekt Messungen könnten weitere Informationen über diesen Zustand ermöglichen.

Bisher liegt kein Modell vor, dass die simultane Anwesenheit von lokalisierten und bandartigen Zuständen an den Versetzungen beschreibt. Ein solches Modell wäre für den hier vorliegenden Fall unter anderem deswegen nötig, da es sich bei den mit der C-Linie assoziierten Defekten um lokalisierte Zustände an den Versetzungen handelt. Außerdem kann nicht ausgeschlossen werden, dass mit den goldinduzierten Zuständen an den Versetzungen sowohl lokalisierte als auch bandartige Zustände vorliegen, die untereinander und mit den C-Liniendefekten koppeln. Interessant ist auch die Frage nach der Leitfähigkeit entlang der Versetzungen mit goldinduzierten bandartigen Zuständen entlang des Versetzungskerns. Theoretische Untersuchungen mit der Annahme hoher Liniendichten von Goldatomen könnten darüber ebenso Aufschluss geben wie die Untersuchung der Mikrowellenleitfähigkeit.

Die Gesamtgoldkonzentration in versetzungshaltigem Silizium ist mit der elektrischen Messmethode DLTS nicht zugänglich. Daher konnten zur versetzungsdichteabhängigen Golddiffusion lediglich Vermutungen angestellt werden. Wie erwähnt, gab es deutliche Abweichungen in den Konzentrationsprofilen der beiden Proben, deren Versetzungsdichte sich um etwa einen Faktor von nur 2.5 unterschied. Dieser Effekt lässt sich folgendermaßen zusammenfassend beschreiben: In Proben mit kleinen Versetzungsdichten ist ein deutlich tieferes Eindringen substitutioneller Goldatome zu beobachten. Darüber hinaus zeigten die in größeren Tiefen gemessenen Zustände dieser Spezies eine im Vergleich zu versetzungsfreiem Silizium unveränderte Einfangkinetik. Die Ursachen für diesen Effekt sind unklar: Möglicherweise erfolgt in Proben mit großen Versetzungsdichten zunächst der Einbau von Goldatomen in den Versetzungskern, während in Proben mit kleinen Versetzungsdichten vorrangig auf Goldatome auf substitutionelle Gitterplätze eingebaut werden. Eine andere Möglichkeit wäre, dass die Ausbildung eines bandartigen Akzeptorzustandes im Versetzungskern eine bestimmte Anordnung der Goldatome voraussetzt, die sich nur langsam einstellen kann. Hier können z.B. Untersuchen mittels hochauflösen- 
der Rastertransmissionselektronenmikroskopie (HRSTEM) in Verbindung mit analytischen Techniken (,EELS“: Elektronenenergieverlustspektroskopie, „EDX“: Energiedispersive Röntgenspektrometrie) und chemisch sensitiver Abbildung (Z-Kontrast) Aufschluss über die Verteilung von Goldatomen längs eines Versetzungskernes geben.

Darüber hinaus konnten in Oberflächennähe einer Probe mittels Transmissionselektronenmikroskopie Goldausscheidungen nachgewiesen werden, die während der Eindiffusion entstanden sein mussten. Diese Ausscheidungen wurden ebenfalls in der Arbeit von Rodriguez und Mitarbeitern ${ }^{[20]}$ gefunden. Ohne die Bildung der Ausscheidungen systematisch untersucht zu haben, wurde ihr Auftreten in der vorliegenden Arbeit als Ursache transienter Prozesse gedeutet, die möglicherweise auf Nichtgleichgewichte intrinsischer Punktdefekte zurückzuführen sind.

Daraus lassen sich Vorschläge für zukünftige Experimente zur Beschreibung von Wechselwirkungen extrinsischer Punktdefekte und Versetzungen ableiten: Auf einer Zeitskala, auf der die Dichte von Versetzungen als konstant angesehen werden kann, sollten intrinsische Punktdefekte im Gleichgewicht mit den Versetzungen stehen, um die erwähnten transienten Prozesse auszuschließen. Dies erfordert die Auslagerung von verformten Proben vor dem Einbringen metallischer Verunreinigungen. Außerdem könnten DLTSExperimente Aufschluss über den Einfluss von Verunreinigungen auf die mit den Versetzungen assoziierten Defekte (C-Linie) bringen, die nach einer Auslagerung unter den Bedingungen der Eindiffusion und ohne Kontamination mit metallischen Verunreinigungen durchzuführen wären. In dieser Arbeit konnte lediglich beobachtet werden, dass zum einen die DLTS-Linienamplitude der C-Linie in der Probe mit der höheren Versetzungsdichte unabhängig von der Goldkonzentration war, während diese in der Probe mit der geringeren Versetzungsdichte in geringem Maße mit der Goldkonzentration abnahm.

Auch in der p-dotierten versetzungshaltigen Probe konnte eine Auffälligkeit in den DLTS-Spektren beobachtet werden: Die Abnahme der Konzentration substitutionellen Goldes ging einher mit der Zunahme versetzungsinduzierter Defekte. Dieser Zusammenhang konnte nicht geklärt werden. Zur Deutung dieses Effektes könnten Experimente beitragen, bei denen zum einen die Versetzungsdichte und zum anderen die Bedingungen der Eindiffusion variiert werden. 
Anhänge 



\section{A Modell zur Berechnung von Punktdefektkonzentrationen}

In der Beschreibung der Umladeprozesse während der Einfang- und Emissionsphase im Verlauf einer Messperiode der DLTS wurden im Kap. 2.1.1 zwei vereinfachende Annahmen gemacht:

1. Die Umladung von tiefen Zuständen findet in der gesamten Raumladungszone unabhängig vom Ort statt.

2. Die Dichte der tiefen Zustände ist klein im Vergleich zur Dichte der flachen Dotierung $\left(N_{\mathrm{T}} \ll N_{\mathrm{d} / \mathrm{a}}\right)$.

Im folgenden sollen am Beispiel eines n-dotierten Halbleiters die Korrekturen erläutert werden, die sich ergeben, wenn diese Annahmen nicht getroffen werden können. PoNS $\underline{\text { [23] }}$ zeigt dazu eine detaillierte Rechnung für Punktdefekte, bei denen der Einfang und die Emission von Minoritätsladungsträgern vernachlässigt werden kann.

In der Abb. A.1 wird ersichtlich, an welchen Stellen die Besetzung der tiefen Zustände in der Raumladungszone vom Ort abhängen. Während der Einfangphase werden ungela-
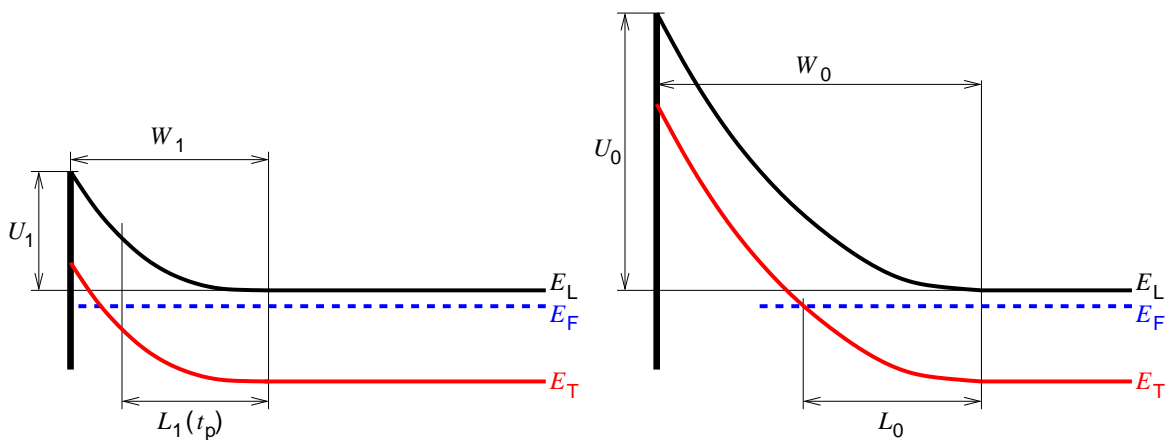

Abbildung A.1 - Die Raumladungszone eines n-dotierten Halbleiters mit dem Potential $U_{1}$ am Rand der Raumladungszone während (links) und mit dem Potential $U_{0}$ zwischen den Ladungspulsen (rechts). $W_{1}$ und $W_{0}$ bezeichnen die entsprechenden Ausdehnungen der Raumladungszone und $E_{\mathrm{L}}, E_{\mathrm{F}}$ und $E_{\mathrm{T}}$ die Leitungsbandkante, das FermiNiveau und die Energie der tiefen Störstelle. In dem mit $L_{1}$ bezeichnete Bereich können noch Ladungsträger eingefangen werden. $L_{0}$ zeigt den Bereich, in dem während der Emissionsphase weiterhin Ladungsträger eingefangen werden. 
denen Punktdefekte im neutralen Bereich bis $W_{1}$ mit der Einfangrate $c_{n} N_{d}$ umgeladen. Innerhalb der Raumladungszone ist die Einfangrate $c_{n} n(x)$, wobei $n(x)$ vom Potential $U_{1}$ abhängt. Der Bereich $W_{1}-L_{1}\left(T_{\mathrm{p}}\right)$, bis zu dem die Punktdefekte Ladungsträger einfangen können, hängt dann logarithmisch von der Einfangzeit $t_{\mathrm{p}}$ ab. Darüber hinaus hängt die Besetzung der tiefen Zustände, aus denen in der Emissionsphase Ladungsträger emittiert werden, am Ende des Ladungspulses ebenfalls von $t_{\mathrm{p}}$ ab, da die Einfangrate vom Einfangquerschnitt limitiert wird. In der Emissionsphase emittieren die Ladungsträger aus den besetzten Zuständen bis zu der Stelle $W_{0}-L_{0}$ in der Weise, wie es im Kap. 2.1.1 beschrieben ist. An dieser Stelle schneidet das Energieniveau der tiefen Störstelle das Quasi-Fermi-Niveau der Majoritätsladungsträger, das innerhalb der Raumladungszone als konstant angenommen werden darf를. Im Bereich zwischen $W_{0}-L_{0}$ und $W_{0}$ nähert sich die Ladungsträgerdichte der Dotierdichte an, so dass es hier einen Beitrag des Ladungsträgereinfang auch in der Emissionsphase gibt („Ladungsträgereinfang aus dem Debye-Tail"). Somit hängt der Anteil an tiefen Zuständen, die in der DLTS innerhalb der Raumladungszone umgeladen werden können, von der Lage des Energieniveaus in der Bandlücke ab. Das Signal des Kapazitätstransienten $\Delta C(t)$ berechnet sich aus dem Integral über die ortsabhängige Besetzungsänderung während der Emission $\Delta f(x, t)$ gemäß

$$
\begin{aligned}
\left(\frac{C_{0}^{2}}{\left(\Delta C(t)+C_{0}\right)^{2}}-1\right) \cdot N_{\mathrm{d}} & =\frac{2 N_{\mathrm{T}}}{W_{0}^{2}} \int_{0}^{l} \Delta f(x, t) x \cdot \mathrm{d} x, \\
& \left.\approx-\frac{2 \Delta C(t)}{C_{0}} \cdot N_{\mathrm{d}} \quad \text { (erste Näherung für } N_{\mathrm{T}} \ll N_{\mathrm{d}}\right)(\text { A. } .2)
\end{aligned}
$$

wobei hier die Konzentrationen sowohl der flachen Dotieratome als auch der tiefen Zustände als homogen vorausgesetzt werden. Die Integrationsgrenze $l$ ist ein Ort im neutralen Halbleiter, an dem das Potential auf Null abgefallen ist. Mit den ortsabhängigen Ladungsträgerdichten $n_{1}(x)$ während der Einfang- und $n_{0}(x)$ während der Emissionsphase und $f_{i}(x, t \rightarrow \infty)=f_{i, \infty}(x)$ wird bei Vernachlässigung der Minoritätsladungsträger aus der Lösung der Ratengleichung Gl. (2.5)

$$
\begin{aligned}
& f_{1}(x, t)=f_{1, \infty}(x)+\left(f_{1}(x, 0)-f_{i, \infty}(x)\right) \exp \left(-\left(e_{n}+c_{n} n_{1}(x)\right) t\right) \\
& f_{0}(x, t)=f_{0, \infty}(x)+\left(f_{0}(x, 0)-f_{i, \infty}(x)\right) \exp \left(-\left(e_{n}+c_{n} n_{0}(x)\right)\left(t-t_{\mathrm{w}}\right)\right)
\end{aligned}
$$

$t_{\mathrm{w}}$ stellt die Periode der Pulswiederholung dar. Die Besetzungen zu Beginn einer Phase entspricht der Besetzung am Ende der jeweils anderen Phase. Daher sind $f_{0}(x, 0)=$ $f_{1}\left(x, t_{\mathrm{p}}\right)$ und $f_{1}(x, 0)=f_{0}\left(x, t_{\mathrm{w}}-t_{\mathrm{p}}\right)$. Daraus ergibt sich folgende Darstellung für die 
Änderung der Besetzung zu Beginn und am Ende der Emissionsphase $\Delta f\left(x, t_{\mathrm{p}}\right)$ :

$$
\begin{aligned}
\Delta f\left(x, t_{\mathrm{p}}\right) & =f\left(x, t_{\mathrm{p}}\right)-f_{0}(x, 0) \\
& =\left(f_{1, \infty}(x)-f_{0, \infty}(x)\right) \frac{1-A_{1}(x)}{1-A_{1}(x) A_{0}(x)}=: R\left(t_{\mathrm{p}}\right) \quad \text { mit } \\
A_{1}(x) & =\exp \left(-\left(e_{n}+c_{n} n_{1}(x)\right) t_{\mathrm{p}}\right) \quad \text { und } \\
A_{0}(x) & =\exp \left(-\left(e_{n}+c_{n} n_{0}(x)\right)\left(t_{\mathrm{w}}-t_{\mathrm{p}}\right)\right)
\end{aligned}
$$

In der Abb. A.2 ist $\Delta f\left(x, t_{\mathrm{p}}\right)$ über den Ort in der Raumladungszone für verschiedene $T_{\mathrm{p}}$ gerechnet. Die Parameter entsprechend denen, die für den Goldakzeptor in n- und den -donator in p-dotiertem Silizium in Kap. 4.2.1 ermittelt wurden. Für die Berechnungen

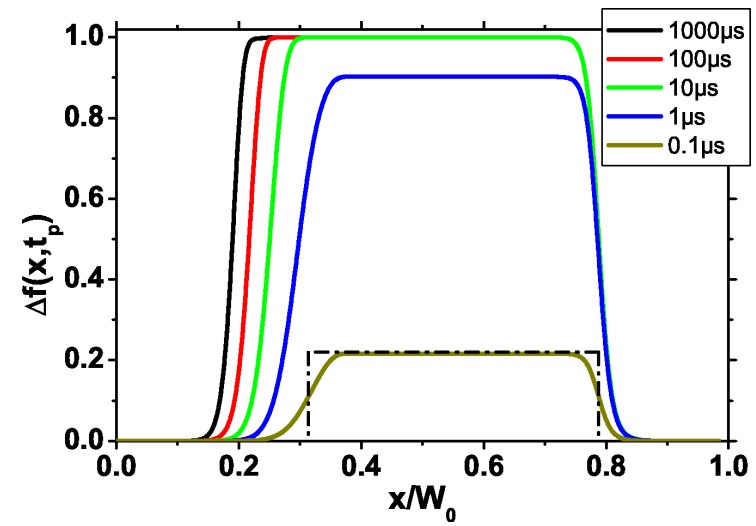

(a) n-dotiertes Silizium, Parameter des GoldAkzeptorzustandes (Tab. 4.2)

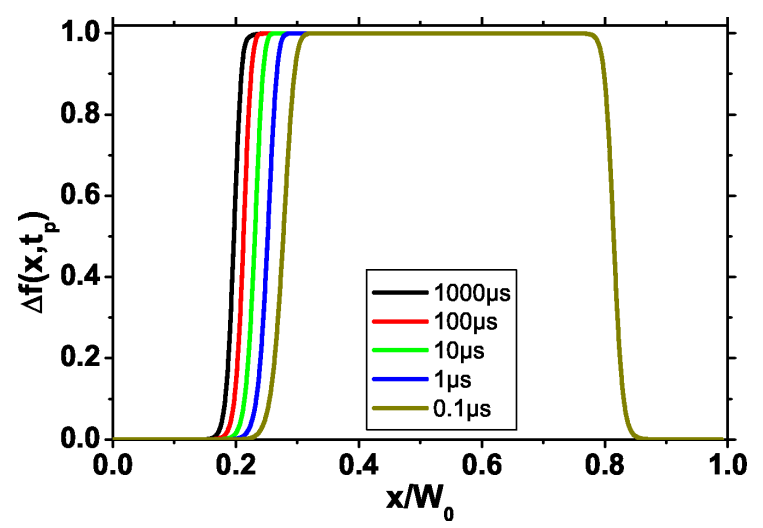

(b) p-dotiertes Silizium, Parameter des GoldDonatorzustandes (Tab. 4.3

Abbildung A.2 - Anteil der während einer Periode umgeladenen tiefen Zustände über den Ort in der Raumladungszone für unterschiedliche $t_{\mathrm{p}}$. Die Parameter waren $U_{\mathrm{b}}=-5 \mathrm{~V}$, $U_{\mathrm{p}}=4.9 \mathrm{~V}, t_{\mathrm{w}}=1 / 17 \mathrm{~Hz}$. Durch eine Kastennäherung wie für $0.1 \mu \mathrm{s}$ beim $p$ dotierten Silizium angedeutet, wird das Integral in Gl. eqrefPonsBesetzungEinfang gelöst.

wurde, anders als bei Pons ${ }^{[23]}$, die Raumladungszone durch die Schottky-Näherung, d.h. mit einem an der Raumladungszonengrenze abrupt ansteigende Ladungsträgerkonzentration beschrieben. Bei PONS wird das DLTS-Signal durch Integration nach Gl. (A.2) berechnet, indem $\Delta f(x, t)$ durch eine Kastenfunktion genähert wird. Dabei werden $L_{1}\left(t_{\mathrm{p}}\right)$ und $L_{0}$ für die Stelle berechnet, wo $\Delta f(x, t)$ auf $1 / 2$ abfällt. Die Amplitude wird mit der Annahme berechnet, dass für die Ladungsträgerkonzentrationen zwischen $W_{1}$ und $W_{0}-L_{0}$ zum einen $n_{1}(x)=N_{d}$ und zum anderen $n_{0}(x)=0$ und damit $f_{1, \infty}(x)=1$ und $f_{0, \infty}(x)=0$ gesetzt werden können. Dann vereinfachen sich die Gl. (A.7) und Gl. 
(A.8) $\mathrm{zu}$

$$
\begin{aligned}
& A_{1}^{*}=\exp \left(-\left(e_{n}+c_{n} N_{d}\right) t_{\mathrm{p}}\right) \quad \text { und } \\
& A_{0}^{*}=\exp \left(-e_{n}\left(t_{\mathrm{w}}-t_{\mathrm{p}}\right)\right)
\end{aligned}
$$

und die Amplitude berechnet sich mit Gl. (A.6) zu

$$
R\left(t_{\mathrm{p}}\right)=\frac{1-A_{1}^{*}}{1-A_{1}^{*} A_{0}^{*}} \quad \text { für } \quad W_{1}<x<W_{0}-L_{0}
$$




\section{B Gold im Verzerrungsfeld von Versetzungen}

Es soll die Konzentration von Gold im Verzerrungsfeld der Versetzungen berechnet werden, die sich mit der Konzentration substitutionellen Goldes im ungestörten Siliziumgitter bei $800^{\circ} \mathrm{C}$ im Gleichgewicht befindet. Bei dieser Temperatur wurde die Eindiffusion in dieser Arbeit vorgenommen. Die Diffusion wurde durch Abschrecken mit einer Abkühlrate von ca. $500 \mathrm{~K} / \mathrm{s}$ beendet (vgl. Kap. 3.4), so dass angenommen wird, dass sich das Gleichgewicht bei dieser Temperatur eingestellt hat. Die elastische Wechselwirkungsenergie von Punktdefekten im Verzerrungsfeld von Versetzungen berechnet sich nach Gl. (2.34) gemäß

$$
\begin{aligned}
E_{\mathrm{el}}(r, \Theta) & =A \cdot \frac{\sin \Theta}{r} \mathrm{mit} \\
A & :=4 \mu b \epsilon r_{0}^{3}
\end{aligned}
$$

Die Linien konstanter Wechselwirkungsenergie (Abb. 2.4) sind Kreise mit dem Radius

$$
R_{\mathrm{el}}=\frac{A}{2 \cdot E_{\mathrm{el}}}
$$

Bei einer kleinen Änderung der elastischen Wechselwirkungsenergie von $E_{\mathrm{el}}$ nach $E_{\mathrm{el}}+\mathrm{d} E_{\mathrm{el}}$ ändert sich die Liniendichte $N_{\mathrm{L}, \mathrm{el}}$ von Punktdefekten entlang der Versetzungslinie dann gemäßs

$$
\begin{aligned}
\mathrm{d} N_{\mathrm{L}, \mathrm{el}} & =n_{\mathrm{pd}} \cdot 2 \pi R_{\mathrm{el}} \cdot \mathrm{d} R_{\mathrm{el}} \\
& =-n_{\mathrm{pd}} \cdot \frac{\pi A^{2}}{2 \cdot E_{\mathrm{el}}^{3}} \cdot \mathrm{d} E_{\mathrm{el}},
\end{aligned}
$$

wobei $n_{\text {pd }}$ durch Gleichung (2.35) gegeben ist. Die Liniendichte der Punktdefekte berechnet sich dann durch Integration über das Energieintervall, das durch die minimale $\left(k_{\mathrm{B}} T\right)$ und maximale Wechselwirkungsenergie $\left(0.85 \mathrm{eV}^{[17]}\right)$ gegeben ist:

$$
N_{\mathrm{L}, \mathrm{el}}=n_{\mathrm{pd}}^{0} \cdot \frac{\pi A^{2}}{2} \int_{k_{\mathrm{B}} T}^{0.85 e V} \frac{\exp \left(\frac{E_{\mathrm{el}}}{k_{\mathrm{B}} T}\right)}{E_{\mathrm{el}}^{3}} \mathrm{~d} E_{\mathrm{el}}
$$




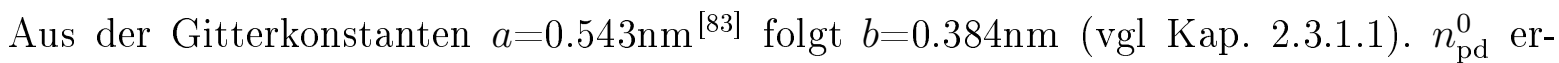
gibt mit Gl. (5.13) und den dort angegebenen Werten $3.94 \cdot 10^{14} \mathrm{~cm}^{-3}$. Mit den Werten $\mu=63.7 \mathrm{GPa}\left({ }^{[13]}, \mathrm{S} .81\right)$ und $r_{0}=0.117 \mathrm{~nm}^{[84]}$ für die elastischen Eigenschaften des Siliziumgitters sowie $\epsilon=0.28^{[17]}$ für die Eigenschaften der Goldatome ergibt Gl. (B.6) $N_{\text {L,el }}=1.4 \cdot 10^{3} \mathrm{~cm}^{-1}$. Bei der Versetzungsdichte von $3.3 \cdot 10^{8} \mathrm{~cm}^{-2}$ ergibt sich eine maximale Löslichkeit von Goldatomen im Verzerrungsfeld von $4.6 \cdot 10^{11} \mathrm{~cm}^{-3}$. Für die Probe mit der Versetzungsdichte von $8 \cdot 10^{8} \mathrm{~cm}^{-2}$ liegt dieser Wert bei $1.1 \cdot 10^{12} \mathrm{~cm}^{-3}$. 


\section{Das Phasendiagramm des Systems Gold/Silizium}

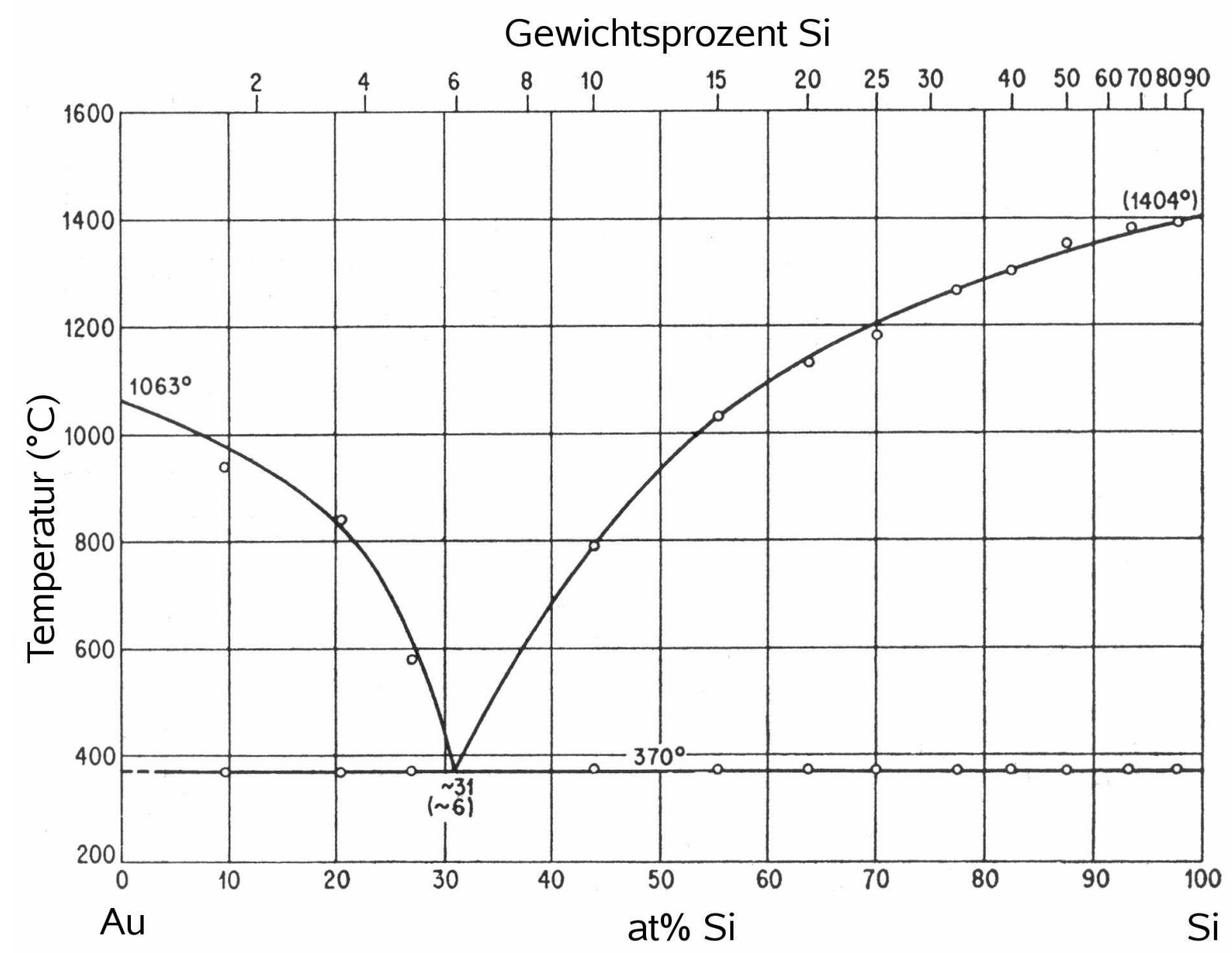

Abbildung C.1 - Phasendiagramm des Systems Gold/Silizium, aus $\underline{\text { [85] }}$. 


\section{Literaturverzeichnis}

[1] S. Hegedus und A. Luque, Handbook of photovoltaic science and engineering: Status, trends, challenges and the bright future of solar electricity from photovoltaics, Kapitel 1, Seiten 1-43, Wiley, 2003.

[2] E. Weber und G. Willeke, Göttinger Physikalisches Kolloquium (2007).

[3] T. Buonassisi, A. A. Istratov, M. D. Pickett, M. Heuer, J. Kalejs, G. Hahn, M. A. Marcus, B. Lai, Z. Cai, S. M. Heald, R. F. Clark, D. W. Cunningham, A. M. Gabor, R. Jonczl, S. Narayanan, E. Sauar und E. R. Weber, Chemical natures and distribution of metal impurities in multicrystalline silicon materials, Prog. Photovolt: Res. Appl. 14, 513 (2006).

[4] C. Rudolf, Transmissionselektronenmikroskopische Untersuchungen zur Koausscheidung von Übergangselementen in kristallinem Silizium, Dissertation, Universität Göttingen, 2009.

[5] A. A. Istratov, T. Buonassisi, R. J. McDonald, A. R. Smith, R. Schindler, J. A. Rand, J. P. Kalejs und E. R. Weber, Metal content of multicrystalline silicon for solar cells and its impact on minority carrier diffusion length, J. Appl. Phys. 94, 6552 (2003).

[6] J. W. Chen und A. G. Milnes, Energy Levels in Silicon, Ann. Rev. Mater. Sci. 10, 157 (1980).

[7] W. Shockley und W. Read, Statistics of the recombinations of holes and electrons, Phys. Rev. 87, 835 (1952).

[8] R. Hall, Electron-hole recombination in germanium, Phys. Rev. 87, 387 (1952).

[9] B. Ceccaroli und O. Lohne, Handbook of photovoltaic science and engineering: Solar grade silicon feedstock, Kapitel 5, Seiten 153-204, Wiley, 2003.

[10] S. M. Myers, M. Seibt und W. Schröter, Mechanisms of transition-metal gettering in silicon, J. Appl. Phys. 88, 3795 (2000).

[11] T. Buonassisi, A. A. Istratov, M. A. Marcus, B. L. andZ. Cai, S. M. Heald und E. R. Weber, Engineering metal-impurity nanodefects for low-cost solar cells, Nature Materials 4, 676 (2005). 
[12] V. Kveder, M. Kittler und W. Schröter, Recombination activity of contaminated dislocations in silicon: A model describing electron-beam-induced current contrast behaviour, Phys. Rev. B 63, 115208 (2001).

[13] W. Schröter und H. Cerva, Interaction of point defects with dislocations in silicon and germanium: Electrical and optical effects, Defect interaction and clustering in semiconductors 85-86, 67 (2002).

[14] N. Fujita, R. Jones, S. Öberg, P. Briddon und A. Blumenau, A theoretical study of copper contaminated dislocations in silicon, Solid State Phenom. 131-133, 259 (2008).

[15] N. Fujita, A. Blumenau, R. Jones, S. Öberg und P. Briddon, Theoretical Study of gold trapped at dislocations in silicon, Poster für die ICDS-Konferenz, Toronto, 2007.

[16] M. Seibt, D. Abdelbaray, V. Kveder, C. Rudolf, P. Saring, L. Stolze und O. Voß, Interaction of metal impurities with extended defects in crystalline silicon and its implications for gettering techniques used in photovoltaics, Mat. Sci. Eng. B, akzeptiert (2009).

[17] R. Bullough und R. Newman, The interaction of impurities with dislocations in silicon and germanium, Progress in Semiconductors, Heywood\&Co., Ltd., London , 103 (1962).

[18] W. Schröter, H. Hedemann, V. Kveder und F. Riedel, Measurements of energy spectra of extended defects, J. Phys.: Condens. Matter 14, 13047 (2002).

[19] R. Labusch, The true potentials and transition barriers at dislocations, J. Phys.: Condens. Matter 14, 12801 (2002).

[20] A. Rodriguez, H. Bracht und I. Yonenaga, Impact of high B concentrations and high dislocation densities on Au diffusion in Si, J. Appl. Phys. 95, 7841 (2004).

[21] D. Lang, Deep-Level Transient Spectroscopy - New method to characterize traps in semiconductors, J. Appl. Phys. 45, 3023 (1974).

[22] P. Blood und J. Orton, The electrical characterization of semiconductors: Majority carriers and electron states, Academic Press, 1992.

[23] D. Pons, Accurate determination of the free carrier capture kinetics of deep traps by space-charge methods, J. Appl. Phys. 55, 3644 (1984).

[24] W. Schröter und R. Labusch, Electrical properties of dislocations in Ge and Si, phys. stst. sol. 36, 539 (1969). 
[25] V. Shikin und N. Shikina, Charged Dislocations in Semiconductors, phys. stat. sol. (a) 108, 669 (1988).

[26] H. Hedemann, Quantitative Analyse von Kapazitätstransientenspektren ausgedehnter Defekte in Halbleitern, Dissertation, Universität Göttingen, 1995.

[27] I. Queißer, Simulation der Kapazitätstransientenspektroskopie an VielElektronendefekten in Silizium, Diplomarbeit, Georg-August-Universität Göttingen, 1988.

[28] T. Kietzke, Einfluss des elektrischen Feldes auf die elektronischen Eigenschaften von Kupfersilizidteilchen in Silizium, Diplomarbeit, Georg-August-Universität Göttingen, 1999.

[29] J. McKelvey, Solid State and Semiconductor Physics, Harper \& Row, New York, Evanston \& London and JohnWeatherhill, Inc., Tokyo, Harper International, 1966.

[30] N. Stolwijk, J. Holzl, W. Frank, E. R. Weber und H. Mehrer, Diffusion of Gold in Dislocation-Free or Highly Dislocated Silicon Measure by the Spreading-Resistance Technique, Applied Physics A-Materials Science \& Processing 39, 37 (1986).

[31] N. A. Stolwijk, B. Schuster und J. Hölzl, Diffusion of Au in Silicon Studied by Means of Neutron-Activation Analysis and Spreasing-Resistance Measurements, Appl. Phys. A 33, 133 (1984).

[32] U. Gösele, W. Frank und A. Seeger, Mechanism and Kinetics of the Diffusion of Gold in Silicon, Appl. Phys. 23, 361 (1980).

[33] H. Zimmermann und H. Ryssel, Gold and platinum diffusion: The key to the understanding of intrinsic point defect behaviour in silicon, Appl. Phys. A 55, 121 (1992).

[34] B. Pichaud, G. Mariani, W. Taylor und W.-S. Yang, Dislocation-gold interaction in silicon, phys. stat. sol. (a) 138, 465 (1993).

[35] R. Mazur und D. Dickey, A spreading resistance technique for resistivity measurements on silicon, J. Electrochem. Soc. 113, 255 (1966).

[36] D. Mathiot, Gold-, self-, and dopant diffusion in silicon, Phys. Rev. B 45, 13345 (1992).

[37] C. Poisson, A. Rolland, J. Bernardini und N. A. Stolwijk, Diffusion of gold into polycrystalline silicon investigated by means of the radiotracer 195Au, J. Appl. Phys. 80, 6179 (1996). 
[38] F. H. Baumann und W. Schröter, Precipitation of gold into metastable gold silicide in silicon, Phys. Rev. B 43, 6510 (1991).

[39] R. W. Carpenter und M. J. Kim, Gold-silicon interfaces: High resolution electron microscopy reactions, precipitation and surface gettering, Mater. Res. Soc. Proc. 104, 153 (1988).

[40] M. Legros, G. Dehm, E. Arzt und T. J. Balk, Observation of giant diffusivity along dislocation cores, Science 319, 1646 (2008).

[41] W. Schröter und M. Seibt, Deep levels of transition metal impurities in c-Si, in Properties of Crystalline Silicon, herausgegeben von R. Hull, Seite 561, INSPEC, 1999.

[42] E. Sveinbjörnsson und O. Engström, Reaction kinetics of hydrogen-gold complexes in silicon, Phys. Rev. B 52, 4884 (1995).

[43] L.Rubaldo, P. Deixler, I. Hawkins, J. Terry, D. Maude, J.-C. Portal, J. EvansFreeman, L. Dobaczewski und A. Peaker, Gold-hydrogen complexes in silicon, Mat. Sci. Eng. B58, 126 (1999).

[44] H. Alexander und H. Teichler, Dislocations, in Handbook of Semiconductor Technology, herausgegeben von K. A. Jackson und W. Schröter, Band 1, Seite 291, WILEY-VCH Weinheim, 2000.

[45] I. Ray und D. Cockayne, Observation of dissociated dislocations in silicon, Phil. Mag. 22, 853 (1970).

[46] J. Rabier und J. Demenet, On the nucleation of shuffle dislocations in Si, phys. stat. sol. (a) 5, 944 (2005).

[47] J. P. Hirth und J. Lothe, Theory of dislocations, KRIEGER PUBLISHING COMPANY MALABAR, FLORIDA, 1982.

[48] W. Read, Statistics of the occupation of dislocation acceptor centers, Phil. Mag. 45, 1119 (1954).

[49] J. Bardeen und W. Shockley, Deformation potentials and mobilities in non-polar crystals, Phys. Rev. 80, 72 (1950).

[50] M. Seibt, V. Kveder, W. Schröter und O. Voß, Structural and electrical properties of metal impurities at dislocations in silicon, phys. stat. sol. (a) 202, 911 (2005).

[51] G. Watkins, Intrinsic point defects in semiconductors, in Materials Science and Technology, herausgegeben von W. Schröter, VCH, 1991. 
[52] G. Samara und C. Barnes, Pressure dependence of impurity levels in semiconductors: The deep gold acceptor level and shallow donor and acceptor levels in silicon, Phys. Rev. B 35, 7575 (1987).

[53] L. Kimerling und J. Patel, Defect states associated with dislocation in silicon, Appl. Phys. Lett. 34, 73 (1979).

[54] P. Omling, E. R. Weber, L. Montelius, H. Alexander und J. Michel, Electrical properties of dislocations and point defects in plastically deformed silicon, Phys. Rev. B 32, 6571 (1985).

[55] D. Cavalcoli, A. Cavallini und E. Gombia, Defect states in plastically deformed n-type silicon, Phys. Rev. B 56, 10208 (1997).

[56] O. Voß, V.V.Kveder, W. Schröter und M. Seibt, Electrical properties of gold at dislocations in silicon, phys. sol. sol. (c) 6, 1847 (2005).

[57] V. Kveder, M. Badylevich, W. Schröter, M. Seibt, E. Steinman und A. Izotov, Silicon light-emitting diodes based on dislocation-related luminescence, phys. stat. sol. (a) 202, 901 (2005).

[58] M. Seibt, R. Khalil, V. Kveder und W. Schröter, Electronic states at dislocations and metal silicide precipitates in crystalline silicon and their role in solar cell materials, Appl. Phys. A, akzeptiert .

[59] C. Kisielowski und E. Weber, Inhomogeneities in plastically deformed silicon single crystals. II. Deep-level transient spectroscopy investigations of $p$ - and $n$-doped silicon, Phys. Rev. B 44, 1600 (1991).

[60] O. Voß, Elektrische Eigenschaften von Gold in plastisch verformtem Silizium, Diplomarbeit, Universität Göttingen, 2003.

[61] R. Khalil, Electrical properties of iron-doped silicon at different stages of precipitation, Dissertation, Universität Göttingen, 2004.

[62] H. Alexander und P. Haasen, Dislocations and Plastic Flow in the Diamond Structure, Solid State Physics 22, 27 (1968).

[63] F. d'Aragona, Dislocation etch for (100) planes in silicon, J. Electrochem. Soc. 119, 948 (1972).

[64] M. Seibt, Elektronenmikroskopische Untersuchung des Ausscheidungsverhaltens von Nickel in Silizium, Dissertation, Universität Göttingen, 1986.

[65] M. von Heimendahl, Einführung in die Elektronenmikroskopie, Vieweg, 1970. 
[66] L. Reimer, Transmission Electron Microscopy, Springer, 1984.

[67] J. Spence, Experimental High-Resolution Electron Microscopy, Oxford University Press, 1988.

[68] D. Williams und C. Carter, Transmission Electron Microscopy, Plenum Press, New York, 1996.

[69] J. Davidson und J. Evans, Electron and hole capture kinetics at gold-hydrogen complexes in n-type silicon, Semicond. Sci. Technol. 11, 1704 (1996).

[70] S. Brotherton und J. Bicknell, The electron capture cross section and energy level of the gold acceptor center in silicon, J. Appl. Phys. 49(2), 667 (1978).

[71] H. Lemke, Dotierungseigenschaften von Silber in Silizium, Phys. Stat. Sol. a 94, K55 (1986).

[72] D. Lang, H. Grimmeis, E. Meijer und M. Jaros, Complex nature of gold-related deep levels in silicon, Phys. Rev. B 22, 3917 (1980).

[73] R. Wu und A. Peaker, Capture cross sections of the gold donor and acceptor states in n-type Czochralski silicon, Solid-State Electron. 28, 643 (1982).

[74] J. Utzig, Elektrotransport von Übergangselementen in Silizium, Dissertation, GeorgAugust-Universität Göttingen, 1985.

[75] G. Zoth, DLTS-Messungen an Gold in Silizium, Diplomarbeit, Universität Göttingen, 1980.

[76] V. Kveder, persönliche Mitteilung (2009).

[77] A. Sattler, Ratenlimitierende Prozesse beim Gettern von Kobalt in Silizium, Dissertation, Universität Göttingen, 2002.

[78] H. Veth und M. Lanoo, The electronic properties of charged dislocations in semiconductors, Phil. Mag. B 50, 93 (1984).

[79] O. Voß, V. Keder und M. Seibt, Electrical properties of gold in dislocated silicon, phys. stat. sol (a) 7, 2185 (2007).

[80] W. Schröter und M. Seibt, Solubility and diffusion of transition metal impurities in c-Si, in Properties of crystalline silicon, R. Hull, 1998.

[81] P. Haasen, Physikalische Metallkunde, Springer-Verlag, 1974.

[82] S. Sze, Physics of Semiconductor Devices, 2nd Edition, John Wiley \& Sons, 1981. 
[83] Y. Okada, Diamond cubic Si: structure, lattice parameter and density, in Properties of crystalline silicon, herausgegeben von R. Hull, Seite 91, INSPEC, 1998.

[84] A. George, Elastic constants and moduli of diamond cubic Si, in Properties of crystalline silicon, herausgegeben von R. Hull, Seite 98, INSPEC, 1997.

[85] M. Hansen, Constitution of binary alloys, Metallurgy and metallurgical engineering series, McGraw-Hill Book Company, 1958. 


\section{Danksagung}

Ich danke meinem Doktorvater Herrn Prof. Dr. M. Seibt für die Ermöglichung dieser Arbeit, für seine stetige Diskussionsbereitschaft, seine wertvolle Kritik, Hilfe bei der Elektronenmikroskopie und die Gelegenheit zu internationalen Tagungen fahren und dort Vorträge halten zu können. Die Erfahrungen während der Arbeit in seiner Arbeitsgruppe waren wichtig und vielseitig nicht nur in fachlicher Hinsicht.

Herrn Prof. Dr. C. Jooß danke ich für sein Interesse an meiner Arbeit und die bereitwillige Übernahme des Korreferats.

Bei Herrn Prof. Dr. W. Schröter möchte ich mich ganz besonders für vielen inspirierenden Diskussionen und Denkanstöße und für sein Interesse bedanken. Seine Unterstützung hat maßgeblich zum Gelingen der Arbeit beigetragen. Prof. Dr. V. Kveder vom Institut für Festkörperphysik in Chernogolovka danke ich ganz herzlich für die stete Bereitschaft, mich mit Rat und Tat zu unterstützen und dafür, dass er mir zum Teil „maßgeschneiderte" Simulationsprogramme zur Verfügung gestellt hat.

Bei Herrn Prof. Dr. H. Bracht von der Universität Münster bedanke ich mich für die Probe, die er mir freundlicherweise zur Verfügung gestellt hat, und für eine wichtige Veröffentlichung.

Herrn Dr. K. Ahlborn und Dr. K. Thiel sei für Hilfestellung bei Computerproblemen und Herrn Dipl.Ing. (grad.) M. Schrader für technische Hilfe gedankt. Herrn Dr. T. Niermann danke ich für die Hilfestellungen beim Lösen von Problemen mit der Programmierung der DLTS-Software.

Den Mitarbeitern in der Elektrowerkstatt und Feinmechanikwerkstatt Klaus Langohr, Rasit Kösker, Thomas Lehmann und Andreas Juretzko danke ich für die Mitarbeit beim Bau der DLTS-Anlage und für die freundliche Zusammenarbeit.

Allen alten und neuen Mitgliedern der Arbeitsgruppe Seibt sei für das angenehme Arbeitsklima, die freundliche wohlwollende Atmosphäre gedankt. Insbesondere möchte ich mich einer Reihe von Kollegen bedanken, mit denen ich eine lange gemeinsame schöne Zeit in der Arbeitsgruppe hatte: Carsten Rudolf, Karsten Thiel, Tore Niermann 
Carsten Rudolf und Philipp Saring danke ich für das Korrekturlesen und die wertvollen Hinweisen sowie für die Übernahme einiger Aufgaben hinsichtlich des Fortgeschrittenenpraktikums.

Frau Bettina Schlieper-Ludewig möchte ich für die Hilfe und ihren Einsatz bei der Präparation von Proben und für unermüdliches Secco-Ätzen in der Endphase meiner Arbeit danken.

Namentlich erwähnen möchte ich einige Mitglieder der Gruppe Rizzi, bei denen ich mich für die oft sehr spontane Hilfe am Rasterelektronenmikroskop bedanken möchte: Dong Du Mai, Martin Röver, Christian Denker und Boris Landgraf.

Philipp Saring, Henning Schuhmann, Hendrik Bergmann, Doaa Abdel Barey, Philipp Hahne, Marie Aylin Falkenberg, Niels Hildebrand sowie Patrick Peretzki und Patrick Schwager danke ich für die freundliche Atmosphäre in unserer Arbeitsgruppe.

Ich danke meinen Eltern Anne und Benno Voß für die nicht nur materielle Ermöglichung des Studiums und ihnen und meinem Bruder Guido Voß für Zuspruch und Motivation.

Meiner lieben Frau Uta Heinemann danke ich dafür, dass sie immer für mich da war und mir immer mit absoluter Zuverlässigkeit „den Rücken freigehalten“ hat. Sie und meine Kinder Jannis, Piet und Lina mussten einige Entbehrungen insbesondere in der Endphase meiner Arbeit erfahren, die sie tapfer gemeistert haben. Vielen Dank! 


\section{Lebenslauf}

Oliver Voß

Geburtstag:

17. August 1973

Geburtsort:

Oldenburg

Staatsangehörigkeit:

deutsch

Familienstand:

verheiratet mit Uta Heinemann,

Kinder: Jannis Heinemann, Piet Heinemann, Lina Heinemann

1980-1984

Grundschule Großenkneten

1984-1986

Orientierungsstufe Ahlhorn

1986-1990

Gymnasium Großenkneten in Ahlhorn

$1990-1993$

Graf-Anton-Günther-Schule Oldenburg

1993-1996

Facharbeiterausbildung: Kommunikationselektroniker/ Telekommunikationselektronik, Telekom Oldenburg

10/1996-09/1999

Studium der Physik an der Universität Göttingen

$10 / 1999-05 / 2000$

Auslandsstudium am Trinity College Dublin, Irland

$06 / 2000-11 / 2003$

Fortsetzung des Studiums an der Universität Göttingen

$11 / 2003$

Diplom

Titel der Diplomarbeit: „Elektrische Eigenschaften von Gold in plastisch verformtem Silizium“

12/2003-09/2005

Wissenschaftlicher Mitarbeiter des BMU-Projektes „ASiS“

$01 / 2006-11 / 2006$

Wissenschaftlicher Mitarbeiter des von der Volkwagenstiftung geförderten Projektes ,SOBSi“

12/2006-06/2007 Wissenschaftlicher Mitarbeiter des DFG-Projekts ,Selbstorganisiertes Wachstum nanoskaliger Multilagenschichten bei der Kodeposition niederenergetischer Metall- und Kohlenstoffionen"

$07 / 2007-05 / 2009$ Wissenschaftlicher Mitarbeiter des BMU-Projektes "SolarFocus“ 